\title{
Magnetotelluric Data, Mid Valley, Nevada Test Site, Nevada
}

By Jackie M. Williams, Erin L. Wallin, Brian D. Rodriguez, Charles R. Lindsay, and Jay A. Sampson

Open-File Report 2007-1123 


\title{
U.S. Department of the Interior DIRK KEMPTHORNE, Secretary
}

\author{
U.S. Geological Survey \\ Mark D. Myers, Director
}

U.S. Geological Survey, Reston, Virginia 2007

For product and ordering information:

World Wide Web: http://www.usgs.gov/pubprod

Telephone: 1-888-ASK-USGS

For more information on the USGS-the Federal source for science about the Earth, its natural and living resources, natural hazards, and the environment:

World Wide Web: http://www.usgs.gov

Telephone: 1-888-ASK-USGS

Prepared in cooperation with the U.S. Department of Energy, National Nuclear Security Administration Nevada Site Office, Office of Environmental Management under Interagency Agreement DE-AI52-07NV28100.

Suggested citation:

Williams, J.M., Wallin, E.L., Rodriguez, B.D., Lindsey, C.R., and Sampson, J.A., 2007, Magnetotelluric Data, Mid Valley, Nevada Test Site, Nevada: U.S. Geological Survey Open-File Report 2007-1123, 137 p.

Any use of trade, product, or firm names is for descriptive purposes only and does not imply endorsement by the U.S. Government.

Although this report is in the public domain, permission must be secured from the individual copyright owners to reproduce any copyrighted material contained within this report. 


\section{Contents}

Introduction

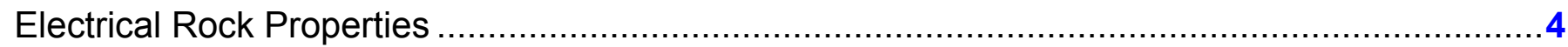

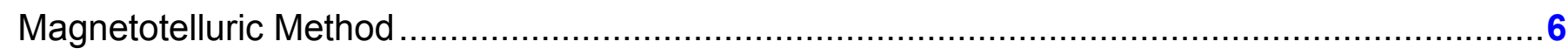

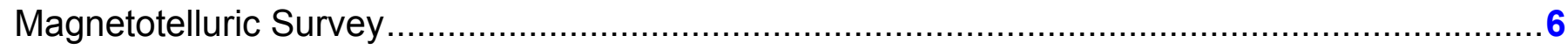

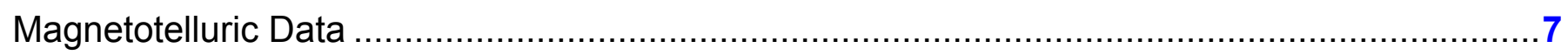

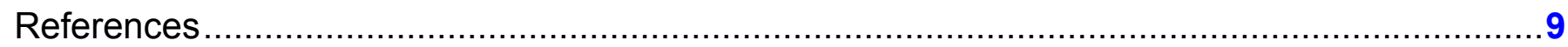

\section{Figure}

1. Index image showing magnetotelluric stations in the Mid Valley Area .................................5 5

\section{Table}

1. Magnetotelluric Station Locations

\section{Appendix}

Magnetotelluric Data Plots 


\section{Introduction}

The United States Department of Energy (DOE) and the National Nuclear Security Administration (NNSA) at their Nevada Site Office (NSO) are addressing ground-water contamination resulting from historical underground nuclear testing through the Environmental Management (EM) program and, in particular, the Underground Test Area (UGTA) project.

One issue of concern is the nature of the somewhat poorly constrained pre-Tertiary geology and its effects on ground-water flow. Ground-water modelers would like to know more about the hydrostratigraphy and geologic structure to support a hydrostratigraphic framework model that is under development for the Rainier Mesa/Shoshone Mountain Corrective Action Unit (CAU) (Bechtel Nevada, 2006).

During 2003, the U.S. Geological Survey (USGS), in cooperation with the DOE and NNSA-NSO, collected and processed data at the Nevada Test Site in and near Yucca Flat (YF) to help define the character, thickness, and lateral extent of the pre-tertiary confining units. We collected 51 magnetotelluric (MT) and audio-magnetotelluric (AMT), stations for that research (Williams and others, 2005a, 2005b, 2005c, 2005d, 2005e, 2005f). In early 2005 we extended that research with 26 additional MT data stations (Williams and others, 2006), located on and near Rainier Mesa and Shoshone Mountain (RM-SM). The new stations extended the area of the hydrogeologic study previously conducted in Yucca Flat. This work was done to help refine what is known about the character, thickness, and lateral extent of pre-Tertiary confining units. In particular, a major goal was to define the upper clastic confining unit (UCCU). The UCCU is comprised of late Devonian to Mississippian siliciclastic rocks assigned to the Eleana Formation and Chainman Shale. The UCCU underlies the Yucca Flat area and extends westward towards Shoshone Mountain, southward to Buckboard Mesa, and northward to Rainier Mesa. Late in 2005 we collected another 14 MT stations in Mid Valley and in northern Yucca Flat basin. That work was done to better determine the extent and thickness of the UCCU near the southeastern RM-SM CAU boundary with the southwestern YF CAU, and also in the northern YF CAU. The purpose of this report is to release the MT data at those 14 stations shown in figure 1. No interpretation of the data is included here.

\section{Electrical Rock Properties}

Electromagnetic geophysical methods detect variations in the electrical properties of rocks-in particular, electrical resistivity, or its inverse, electrical conductivity. Electrical resistivity can be correlated with geologic units on the surface and at depth using lithologic logs to provide a

three-dimensional (3-D) picture of subsurface geology. In the upper crust the resistivity of geologic units is largely dependent upon their fluid content, pore-volume porosity, interconnected fracture porosity, and conductive mineral content (Keller, 1989). Although there is not a one-to-one relationship between lithology and resistivity, there are general correlations that can be made using typical resistivity values, even though values vary from one location to another (Palacky, 1987). Fluids within the pore spaces and fracture openings, especially if saline, can reduce resistivities in what would otherwise be a resistive rock matrix. Resistivities can also be lowered by the presence of electrically conductive clay minerals, graphitic carbon, and metallic mineralization. It is common, for example, for altered volcanic rocks to contain replacement minerals that have resistivities ten times lower than those of the surrounding rocks (Nelson and Anderson, 1992). Fine-grained sediments, such as clay-rich alluvium, marine shales, and other mudstones, are normally conductive from a few ohm-meters (ohm-m) to a few tens of ohm-m (Keller, 1987; Palacky, 1987). Metamorphic rocks (non-graphitic) and unaltered, unfractured igneous rocks are normally moderately to highly resistive (a few hundreds to thousands of ohm-m). Carbonate rocks can have similarly high resistivities depending on their fluid content, porosity, and impurities 
(Keller, 1987; Palacky, 1987). Fault zones may be moderately conductive (tens of ohm-m) when comprised of rocks fractured enough to have hosted fluid transport and consequent mineralogical alteration (Eberhart-Phillips and others, 1995). Higher subsurface temperatures cause higher ionic mobility that reduces rock resistivities (Keller, 1987; Palacky, 1987). Tables of electrical resistivities for a variety of rocks, minerals, and geological environments may be found in Keller (1987) and Palacky (1987).

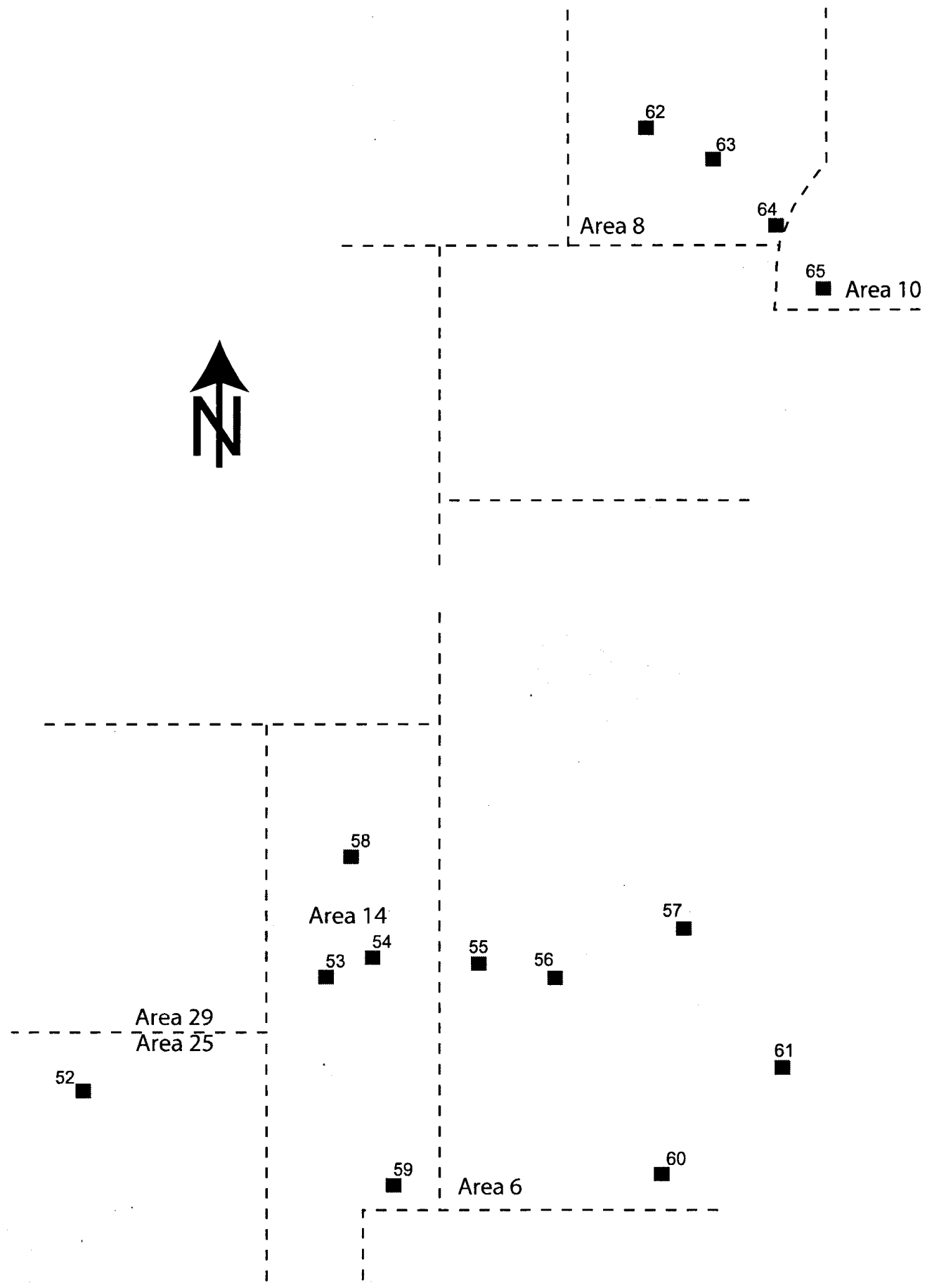

Figure 1. Index image showing magnetotelluric stations in the Mid Valley Area. 


\section{Magnetotelluric Method}

The MT method is a passive-surface electromagnetic geophysical technique that measures variations in the Earth's natural electromagnetic fields to investigate the electrical resistivity structure of the subsurface from depths of tens of meters to tens of kilometers (Vozoff, 1991). Worldwide lightning activity at frequencies of 10,000 to $1 \mathrm{Hertz}(\mathrm{Hz})$ and geomagnetic micro-pulsations at frequencies of 1 to $0.001 \mathrm{~Hz}$ provide the majority of natural signal used by the MT method. The natural electromagnetic wave propagates vertically into the Earth due to the large resistivity contrast between the air and the Earth, causing a vertical refraction of the electromagnetic wave transmitted into the Earth (Vozoff, 1972).

The natural fields are recorded in the xyz direction for the magnetic field and the xy direction for the electric field at the Earth's surface. The resulting time-series signals are used to derive tensor apparent-resistivities and phases by first converting them to complex cross-spectra using

Fourier-transform techniques. Least squares, cross-spectral analysis (Bendat and Piersol, 1971) are used to solve for a tensor transfer function. Prior to conversion to apparent resistivity and phase, the tensor is normally rotated into principal directions that usually correspond to the direction of maximum and minimum apparent resistivity. For a two-dimensional (2-D) Earth, in which Earth's resistivity structure varies with depth and in one lateral direction, the MT fields can be decoupled into transverse-electric (TE) and transverse-magnetic (TM) modes; 2-D resistivity modeling is generally computed to fit both modes. When the geology satisfies the 2-D assumption, the MT data for the TE mode represents electric fields that are oriented parallel to geologic strike, and the data for the TM mode represents electric fields oriented perpendicular to strike. The MT method is well suited for studying complicated geological environments because the electric and magnetic fields are sensitive to vertical and horizontal variations in resistivity. The method is capable of establishing whether the electromagnetic fields are responding to subsurface rock bodies of effectively 1,2, or 3 dimensions. An introduction to the MT method and references for a more advanced understanding are contained in Dobrin and Savit (1988) and Vozoff (1991).

\section{Magnetotelluric Survey}

In October of 2005 we collected 14 MT stations around Rainier Mesa and Shoshone Mountain and in northern Yucca Flat (fig. 1). The station locations were chosen to constrain the geologic/hydrostratigraphic interpretation, for proximity to roads, and to avoid, where possible, electrical noise from power lines and vehicles. The MT data were collected with an Electromagnetic Instruments, Inc., (EMI) MT-1 system (EMI, 1996). Horizontal electric fields were measured using three copper/copper sulfate porous-pot electrodes placed in an L-shaped array with dipole lengths of 30 meters $(\mathrm{m})$. The orthogonal, horizontal magnetic fields in the direction of the electric-field measurement array were sensed using EMI's high-magnetic-permeability, mu-metal-cored induction coils. The MT data were recorded as non-remote referenced single stations.

The following table lists the 14 MT station locations as recorded using a GPS during field acquisition. Coordinates are referenced to the 1866 Clarke spheroid and North American 1927 Western United States datum. Longitude and latitude format is degrees, minutes, seconds. Universal Transverse Mercator (UTM) Zone 11 North and East units are in meters. Station elevation is given in meters. The accuracy of the $x, y$ component is $\pm 5 \mathrm{~m}$. The accuracy of the $z$ component is $\pm 10 \mathrm{~m}$. Ex direction is given in degrees and is the direction of the measured magnetic (Hx) and electric (Ex) fields. 
Table 1. Station Locations

\begin{tabular}{|c|c|c|c|c|c|c|}
\hline Station & $\begin{array}{l}\text { Latitude } \\
(\mathrm{d}, \mathrm{m}, \mathrm{s})\end{array}$ & $\begin{array}{l}\text { Longitude } \\
\qquad(\mathrm{d}, \mathrm{m}, \mathrm{s})\end{array}$ & $\begin{array}{c}\text { Elevation } \\
\text { (m) }\end{array}$ & $\begin{array}{c}\text { Northing } \\
\text { (m) }\end{array}$ & $\begin{array}{c}\text { Easting } \\
(\mathrm{m})\end{array}$ & $\begin{array}{l}\text { Ex } \\
\text { dir }\end{array}$ \\
\hline 52 & $36,54 ' 21.2$ & $116,14^{\prime} 50.5$ & 1596 & 4084493 & 567053 & 180 \\
\hline 53 & $36,55^{\prime} 43.0$ & $116,10 ' 59.3$ & 1460 & 4087063 & 572750 & 0 \\
\hline 54 & $36,55^{\prime} 57.4$ & $116,10^{\prime} 14.3$ & 1400 & 4097522 & 573860 & 0 \\
\hline 55 & $36,55^{\prime} 50.9$ & $116,08^{\prime} 40.3$ & 1321 & 4087336 & 576187 & 180 \\
\hline 56 & $36,55^{\prime} 49.7$ & $116,07^{\prime} 17.8$ & 1381 & 4087314 & 578229 & 0 \\
\hline 57 & $36,56 ' 29.0$ & $116,05^{\prime} 16.8$ & 1436 & 4088553 & 581213 & 180 \\
\hline 58 & $36,57^{\prime} 12.9$ & $116,10^{\prime} 31.7$ & 1415 & 4089848 & 573490 & 0 \\
\hline 59 & $36,52 ' 56.8$ & $116,09 ' 59.3$ & 1416 & 4081955 & 574354 & 0 \\
\hline 60 & $36,52^{\prime} 08.6$ & $116,05 ' 37.0$ & 1186 & 4082376 & 580776 & 180 \\
\hline 61 & $36,54^{\prime} 30.8$ & $116,03^{\prime} 43.7$ & 1221 & 4084935 & 583550 & 0 \\
\hline 62 & $37,11 ' 32.4$ & $116,05^{\prime} 55.0$ & 1458 & 4116384 & 580001 & 0 \\
\hline 63 & $37,11^{\prime} 09.1$ & $116,04^{\prime} 50.0$ & 1394 & 4115683 & 581613 & 0 \\
\hline 64 & $37,10^{\prime} 12.6$ & $116,03^{\prime} 42.9$ & 1317 & 4113957 & 583282 & 0 \\
\hline 65 & $37,09 ' 21.4$ & $116,02 ' 53.7$ & 1291 & 4112395 & 584511 & 0 \\
\hline
\end{tabular}

\section{Magnetotelluric Data}

The recorded time-series data were transformed to the frequency domain and processed to determine the impedance tensor, which is used to derive apparent resistivities and phases at each site. Rotation of the impedance tensor allows for decoupling into the TE and TM modes. The data provided here have not been rotated from the original north-south, east-west acquisition orientation. During the analysis and interpretation process, each station will be rotated to a fixed angle determined by the given nominal profile orientation. Cross-power files were sorted to select optimal signal-to-noise time-series data sets (see Appendix 1).

The effects of near-surface resistivity anomalies can cause what are known as "static shifts" in the data (Sternberg and others, 1988). Cultural features also can affect the measured magnetotelluric responses. These features include fences, pipelines, communication lines, railways, and other manmade conductors. 
The figures in appendix 1 represent the field-processed MT data for each station, after the timeseries data were converted to the frequency domain and the tensor-transfer function was developed.

For each station, nine separate plots are given:

Apparent Resistivity ( $\mathrm{x}$ and o symbols are $\mathrm{xy}$ and $\mathrm{yx}$ components)

Impedance Phase ( $\mathrm{x}$ and o symbols are $\mathrm{xy}$ and $\mathrm{yx}$ components)

Rotation Angle

Impedance Skew

Multiple Coherency ( $\mathrm{x}$ and o symbols are $\mathrm{xy}$ and $\mathrm{yx}$ components)

Impedance Polar Plots

Tipper Magnitude

Tipper Strike

HzHx (x symbol) and HzHy (o symbol) Coherency

Error bars (],[) on the Apparent Resistivity, Impedance Phase, Skew, Tipper Magnitude, and Tipper Strike plots represent probable errors within one standard deviation of the sample variance (Gamble and others, 1979).

Apparent resistivity is the approximate ratio of the electric-field strength to the magnetic-field strength at a given frequency. The impedance phase is proportional to the slope of the apparent resistivity curve on a log-log plot, but from baselines at \pm 45 degrees (Vozoff, 1991). A measure of the dimensionality for MT data is provided by the impedance skew of the impedance tensor (Vozoff, 1972). If the effective measured resistivity response to the geology beneath a MT station truly is one or two dimensional, then the skew will be zero. Instrumental and environmental sources of electrical noise can cause non-zero skew values. Skew values typically are small (about 0.1 ) for relatively low-noise recordings. Higher skews (above 0.2) are an indication of either the resistivity response to 3-D geology or higher levels of noise. Manmade electrical noise, such as power lines, power generators, and moving vehicles and trains, can have a negative effect on MT data quality. All of these local disturbances can produce incoherent noise that mainly affects frequencies above $1 \mathrm{~Hz}$. Other manmade electrical noise, such as direct-current electric trains and active cathodic protection of pipelines, produces coherent electromagnetic signals that mainly affect frequencies below $1 \mathrm{~Hz}$.

In the survey area, noise from a number of small power lines and small moving vehicles was negligible at distances greater than $0.4 \mathrm{~km}$ from the noise source. Power-line signal levels were measured at each site and typically were less than 20 percent of the maximum recordable signals. Noise from larger power lines, power generators, pipelines, and trains was negligible at distances greater than $5 \mathrm{~km}$. Local lightning, wind, and rainstorms may also degrade data quality. Burying the magnetic induction coils and the electric dipole wires minimized wind noise.

Predicted values of the electric field can be computed from the measured values of the magnetic field (Vozoff, 1991). The coherence of the predicted electric field with the measured electric field is a measure of the signal-to-noise ratio provided in the multiple coherency plots. Values are normalized between 0 and 1; values at 0.5 signify signal levels equal to noise levels. For this data set, coherencies generally were at an acceptable level, except at times in the frequency ranges of about 0.1 to $5 \mathrm{~Hz}$ (traditionally referred to as the "dead band").

The field-processed MT data include some scatter and poor signal-to-noise ratios. Spectral results were inspected visually for noisy data, and the best signal-to-noise field data were combined into the final plots.

The magnetotelluric impedance polar plots provide a measure of MT data dimensionality (Reddy and others, 1977). For 1-D resistivity structures, the principal impedance polar diagram (dashed line) is a circle. For 2-D or 3-D resistivity structures, the principal impedance polar diagram (dashed line) 
elongates either parallel or perpendicular to strike direction. Over resistors, the principal impedance polar diagram elongates perpendicular to strike direction, and over conductors, it elongates parallel to strike direction. For 2-D resistivity structures, the additional impedance polar diagram (solid line) attains the shape of a symmetric clover leaf. For 3-D resistivity structures, the additional impedance polar diagram (solid line) elongates in one direction, and its amplitude is comparable to that of the principal impedance polar diagram (dashed line).

The magnetotelluric "tipper" is calculated from the vertical component of the magnetic field. The tipper magnitude is a measure of the "tipping" of the magnetic field out of the horizontal plane (Vozoff, 1991). It will equal zero for the 1-D case. It typically increases to values between 0.1 to 0.5 and seldom approaches 1 , as it responds primarily to vertical and subvertical structures. The tipper strike is used to help resolve the 90-degree ambiguity in the impedance rotation angle. The HzHx and HzHy coherency is a measure of the signal-to-noise ratio of the vertical magnetic field with respect to each of the orthogonal, horizontal magnetic-field directions. Values are normalized between 0 and 1; values at 0.5 signify signal levels equal to noise levels. These three-component magnetic-field coherencies provide a check on the signal-to-noise ratio of the measured values in the tipper magnitude and tipper strike plots.

\section{References}

Bechtel Navada, 2006, A hydrostratigraphic model and alternatives for the groundwater flow and contaminant transport model of Corrective Action Unit 97: Yucca Flat-Climax Mine, Lincoln and Nye counties, Nevada: Report DOE/NV/11718-1119.

Bendat, J.S., and Piersol, A.G., 1971, Random data analysis and measurement procedures: New York, Wiley Interscience, $407 \mathrm{p}$.

Dobrin, M.D., and Savit, C.H., 1988, Introduction to geophysical prospecting (4th ed.): New York: McGraw-Hill, 867 p.

Eberhart-Phillips, Donna, Stanley, W.D., Rodriguez, B.D., and Lutter, W.J., 1995, Surface seismic and electrical methods to detect fluids related to faulting: Journal of Geophysical Research, v. 100, no. B7, p. $12,919-12,936$.

EMI, Inc., 1996, MT-1 magnetotelluric system operation manual, version 3.2: Richmond, Calif., ElectroMagnetic Instruments, Inc., 220 p.

Gamble, T.D., Goubau, W.M., and Clarke, J., 1979, Error analysis for remote reference magnetotellurics: Geophysics, v. 44, no. 5, p. 959-968.

Keller, G.V., 1987, Rock and mineral properties, in Nabighian, M.N., ed., Electromagnetic methods in applied geophysics theory: Tulsa, Okla., Society of Exploration Geophysicists, v. 1, p. 13-51.

Keller, G.V., 1989, Electrical properties, in Carmichael, R.S., ed., Practical handbook of physical properties of rocks and minerals: Boca Raton, Fla., CRC Press, p. 359-427.

Nelson, P.H., and Anderson, L.A., 1992, Physical properties of ash flow tuff from Yucca Mountain, Nevada: Journal of Geophysical Research, v. 97, no. B5, p. 6,823-6,841. 
Palacky, G.J., 1987, Resistivity characteristics of geologic targets, in Nabighian, M.N., ed., Electromagnetic methods in applied geophysics theory: Tulsa, Okla., Society of Exploration Geophysicists, v. 1, p. 53-129.

Reddy, I.K., Rankin, David, and Phillips, R.J., 1977, Three dimensional modelling in magnetotelluric and magnetic variational sounding: Geophysics Journal of the Royal Astronomical Society, v. 51, p. 313-325.

Sternberg, B.K., Washburne, J.C., and Pellerin, Louise, 1988, Correction for the static shift in magnetotellurics using transient electromagnetic soundings: Geophysics, v. 53, p. 1,459-1,468.

Vozoff, Keeva, 1972, The magnetotelluric method in the exploration of sedimentary basins: Geophysics, v. 37 , p. $980-1041$.

Vozoff, Keeva, 1991, The magnetotelluric method, in Nabighian, M.N., Electromagnetic methods in applied geophysics: Tulsa, Okla., Society of Exploration Geophysicists, v. 2, pt. B, p. 641-711.

Williams, J.M., Rodriguez, B.D., and Asch, T. H., 2005a, Magnetotelluric data, central Yucca Flat, Nevada Test Site, Nevada: U.S. Geological Survey Open-File Report 2005-1238, 174 p. (http://pubs.usgs.gov/of/2005/1238).

Williams, J.M., Rodriguez, B.D., and Asch, T. H., 2005b, Magnetotelluric data, northern Yucca Flat, Nevada Test Site, Nevada: U.S. Geological Survey Open-File Report 2005-1239, 120 p. (http://pubs.usgs.gov/of/2005/1239).

Williams, J.M., Rodriguez, B.D., and Asch, T. H., 2005c, Magnetotelluric data, northern Frenchman Flat, Nevada Test Site, Nevada: U.S. Geological Survey Open-File Report 2005-1240, 156 p. (http://pubs.usgs.gov/of/2005/1240).

Williams, J.M., Rodriguez, B.D., and Asch, T. H., 2005d, Magnetotelluric data, southern Yucca Flat, Nevada Test Site, Nevada: U.S. Geological Survey Open-File Report 2005-1241, 210 p. (http://pubs.usgs.gov/of/2005/1241).

Williams, J.M., Rodriguez, B.D., and Asch, T. H., 2005e, Magnetotelluric data, across Quartzite Ridge, Nevada Test Site, Nevada: U.S. Geological Survey Open-File Report 2005-1242, 174 p. (http://pubs.usgs.gov/of/2005/1242).

Williams, J.M., Rodriguez, B.D., and Asch, T. H., 2005f, Magnetotelluric data, north-central Yucca Flat, Nevada Test Site, Nevada: U.S. Geological Survey Open-File Report 2005-1243, 156 p. (http://pubs.usgs.gov/of/2005/1243).

Williams, J.M., Sampson, J.A., Rodriguez, B.D., and Asch, T. H., 2006, Magnetotelluric data, Rainier Mesa/Shoshone Mountain, Nevada Test Site, Nevada: U.S. Geological Survey Open-File Report 2006-1215, 240 p. (http://pubs.usgs.gov/of /2006/1215). 


\section{Appendix 1. Magnetotelluric Data Plots}

There are nine separate plots for each station:

1. Apparent Resistivity for the $x-y$ direction ( $x$ symbol) and $y-x$ direction (o symbol) modes

2. Impedance Phase for the rotated maximum (x symbol) and minimum (o symbol) modes

3. Rotation Angle for the impedance tensor (corresponds to the $x-y$ direction)

4. Impedance Skew for the impedance tensor

5. Multiple Coherency for the $\mathrm{x}-\mathrm{y}$ direction (x symbol) and $\mathrm{y}-\mathrm{x}$ direction (o symbol) modes of the electric field

6. Impedance Polar Plots (at 12 selected frequencies)

7. Tipper Magnitude for the vertical magnetic field

8. Tipper Strike for the vertical magnetic field

9. HzHx (x symbol) and HzHy (o symbol) Coherency

Refer to the "Magnetotelluric Data" section in this report for an explanation of these plots. The priorities listed on the plots were determined prior to data acquisition. During post-processing the priority ranking was changed. 


\section{Station 52}

APPARENT RESISTIVITY

Shoshone Peak

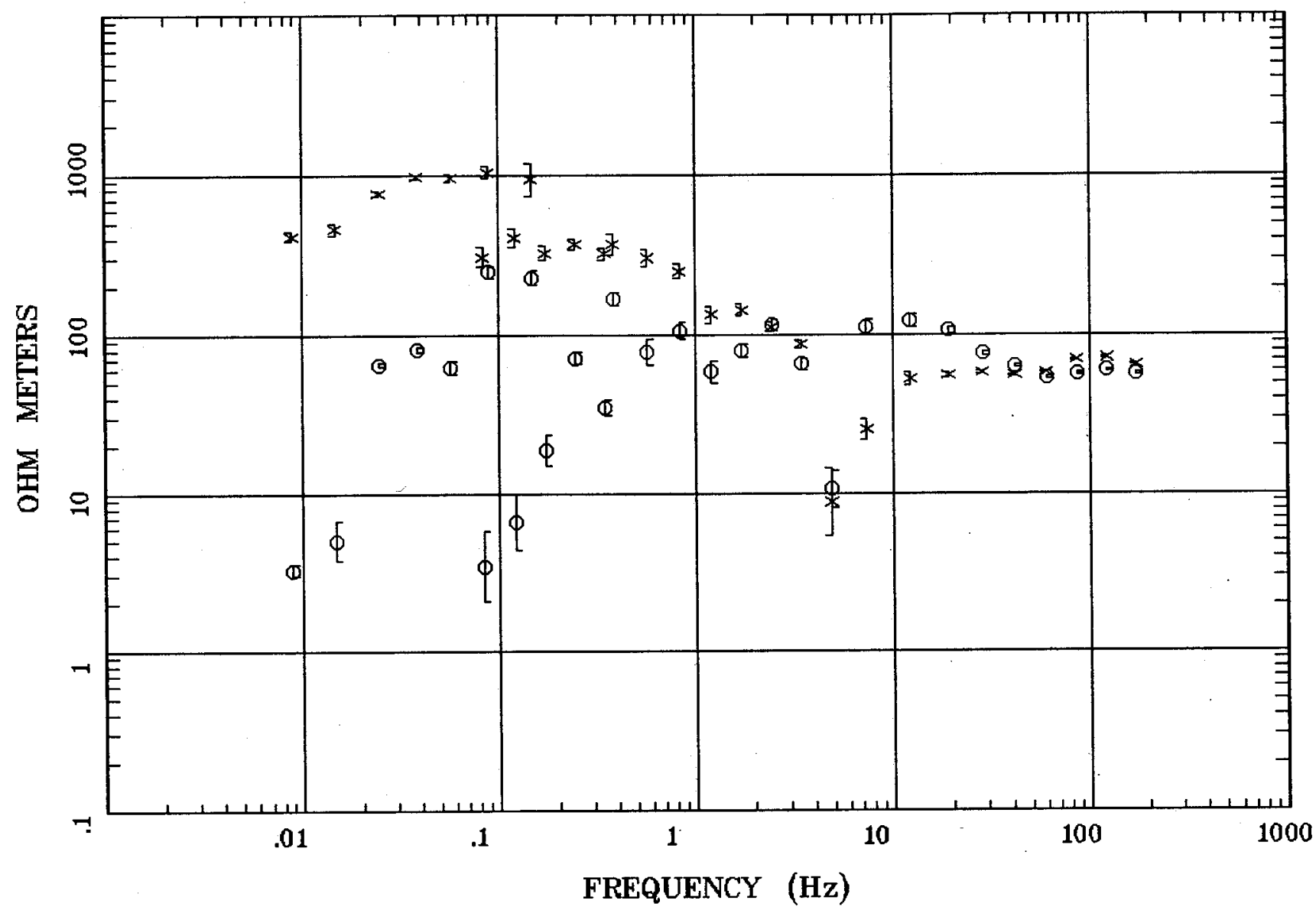

Client: WAter Resources \& DOD

Rotation:

Remote: none

Acquired: $07: 3$ oct 10, 2005

Filename: nt52m.avg

Channels: Ch1 Ch2 Ch3 Ch4 Ch5 Ch3 Ch4

Plotted: 15:09 Feb 08, 2006

Survey Co:US Geological Survey

< EMI - ElectroMagnetic Instruments > 


\section{Station 52}

IMPEDANCE PHASE

Shoshone Peak

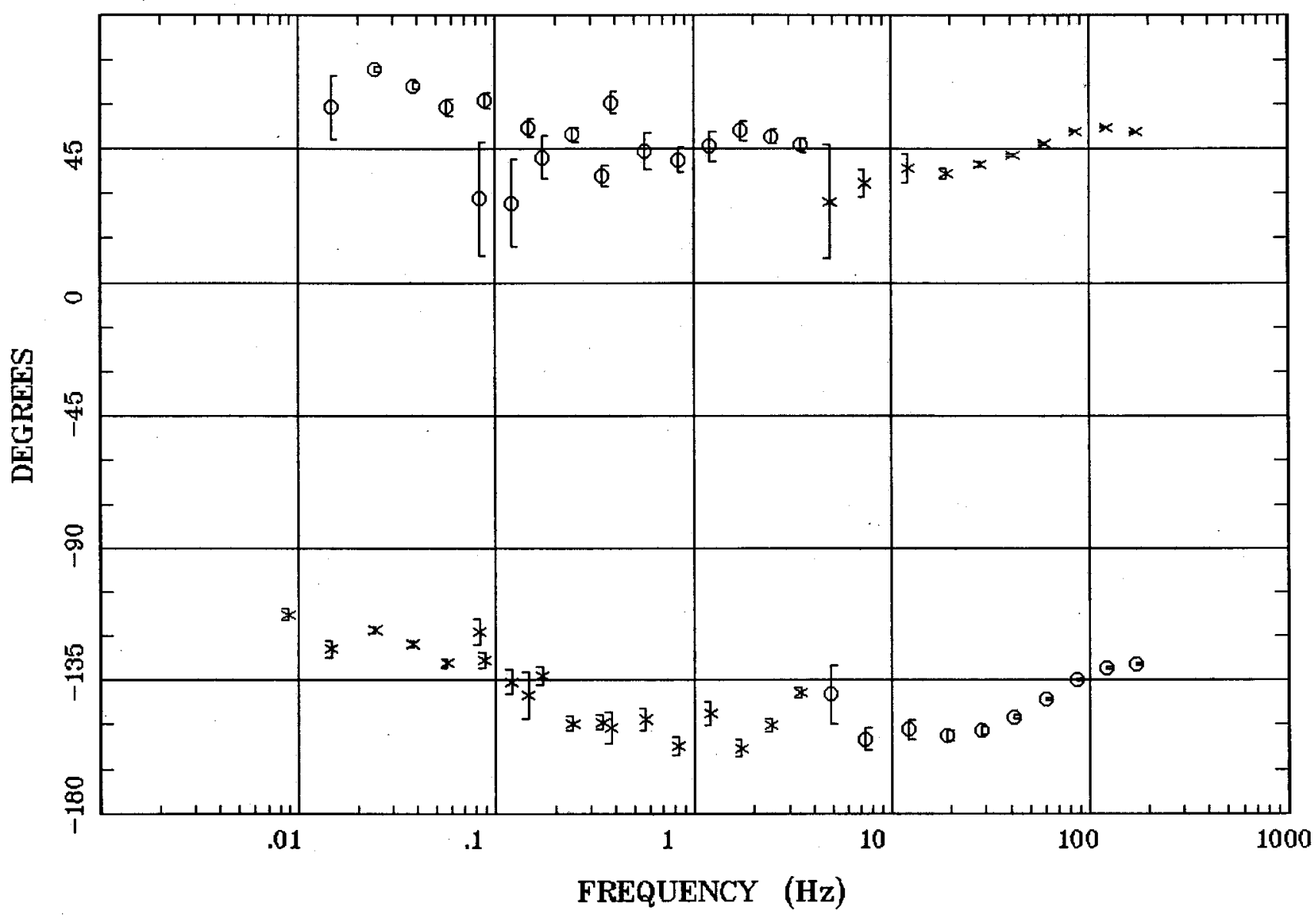

Client: WAter Resources \& DOD Remote: none

Acquired: 07:3 oct 10, 2005 Survey Co:US Geological Survey
Rotation:

Filename: nt52m.avg

Channels: Ch1 Ch2 Ch3 Ch4 Ch5 Ch3 Ch4 Plotted: 15:09 Feb 08, 2006

< EMI - ElectroMagnetic Instruments > 


\section{Station 52}

\section{ROTATION ANGLE}

Shoshone Peak

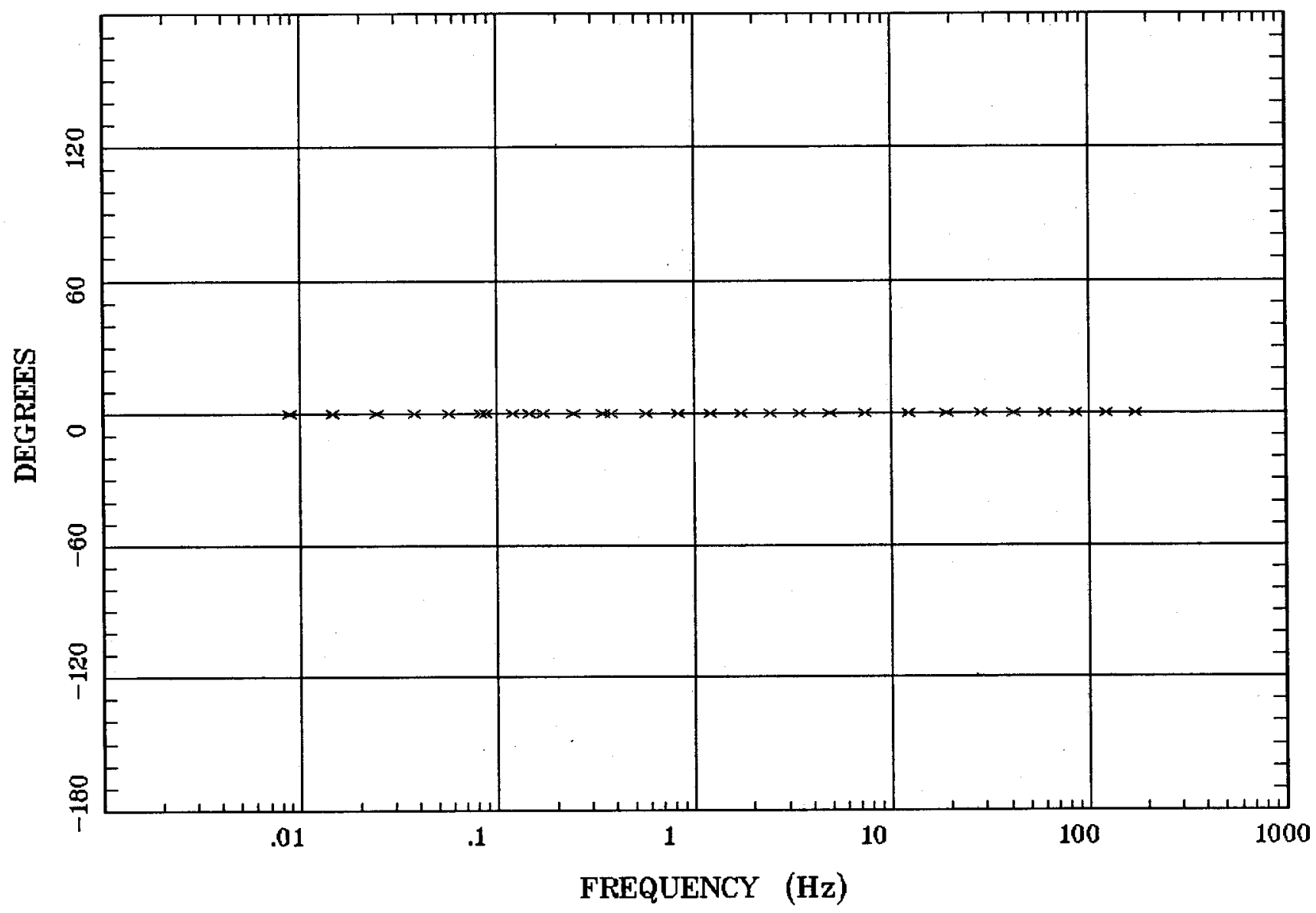

Client: WAter Resources \& DOD

Rotation:

Remote: none

Acquired: $07: 3$ act 10,2005

Survey Co:US Geological Survey

Filename: nt52m.avg

Channels: Ch1 Ch2 Ch3 Ch4 Ch5 Ch3 Ch4

Plotted: 15:09 Feb 08, 2006

< EMI - ElectroMagnetic Instruments > 
Station 52

IMPEDANCE SKEW

Shoshone Peak

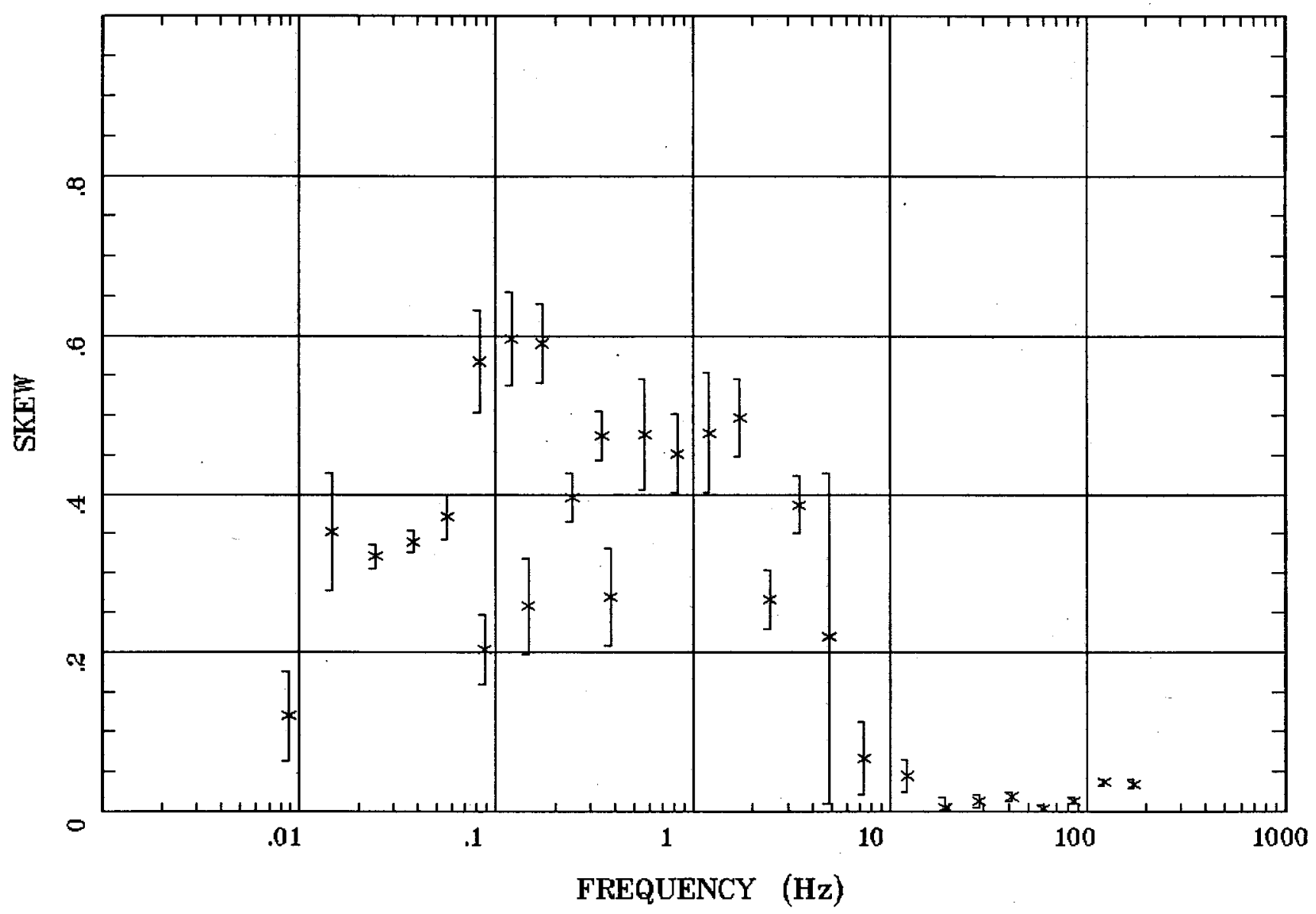

Client: WAter Resources \& DOD Remote: none Acquired: 07:3 oct 10, 2005 Survey Co:US Geological Survey
Rotation:

Filename: nt52m.avg Channels: Ch1 Ch2 Ch3 Ch4 Ch5 Ch3 Ch4 Plotted: 15:09 Feb 08, 2006

< EMI - ElectroMagnetic Instruments > 
Station 52

E MULT Coh. Shoshone Peak

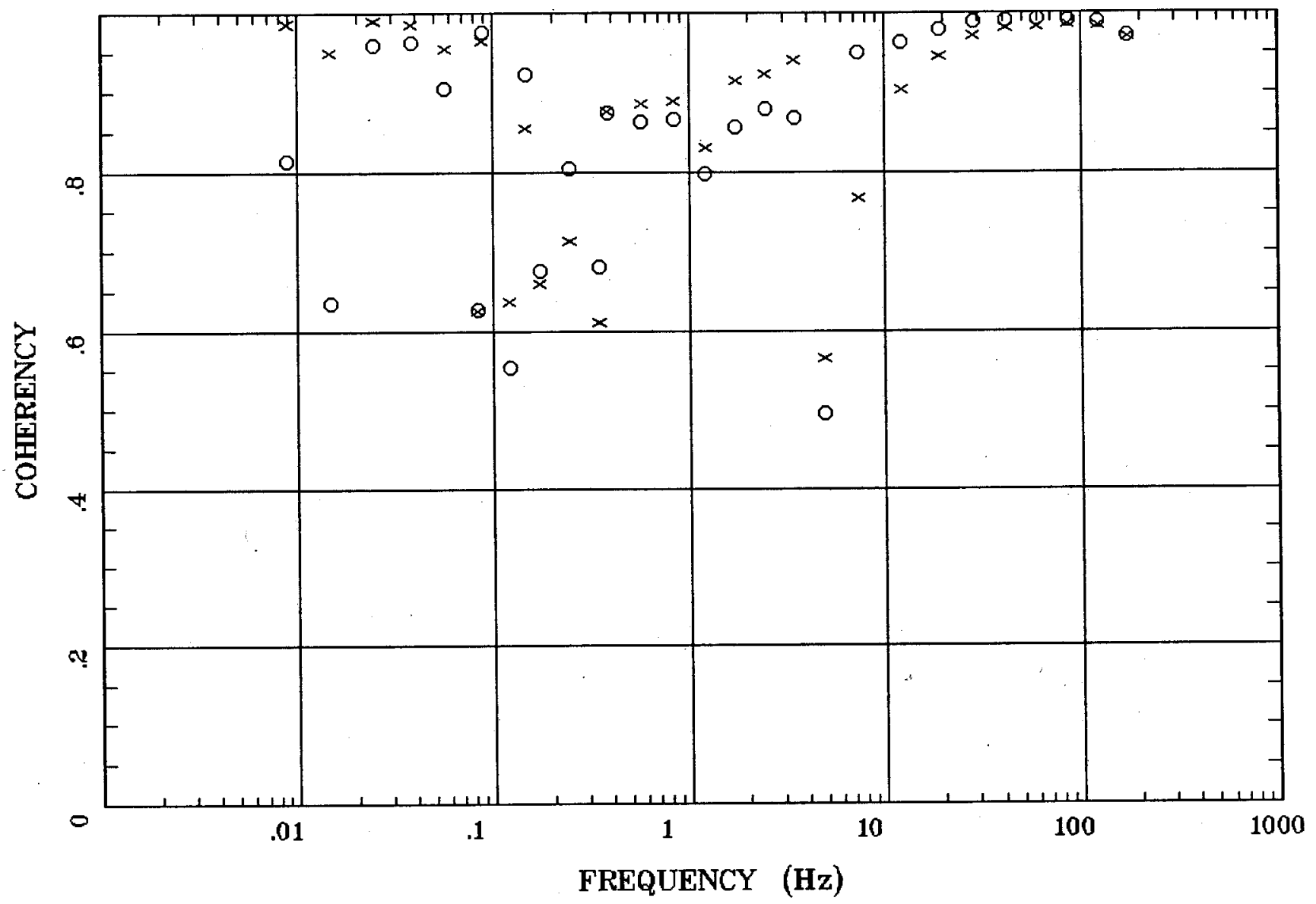

Client: WAter Resources \& DOD

Rotation:

Remote: none

Filename: nt52m.avg

Channels: Ch1 Ch2 Ch3 Ch4 Ch5 Ch3 Ch4

Acquired: 07:3 oct 10, 2005

Plotted: 15:09 Feb 08, 2006

Survey Co:US Geological Survey

< EMI - ElectroMagnetic Instruments > 


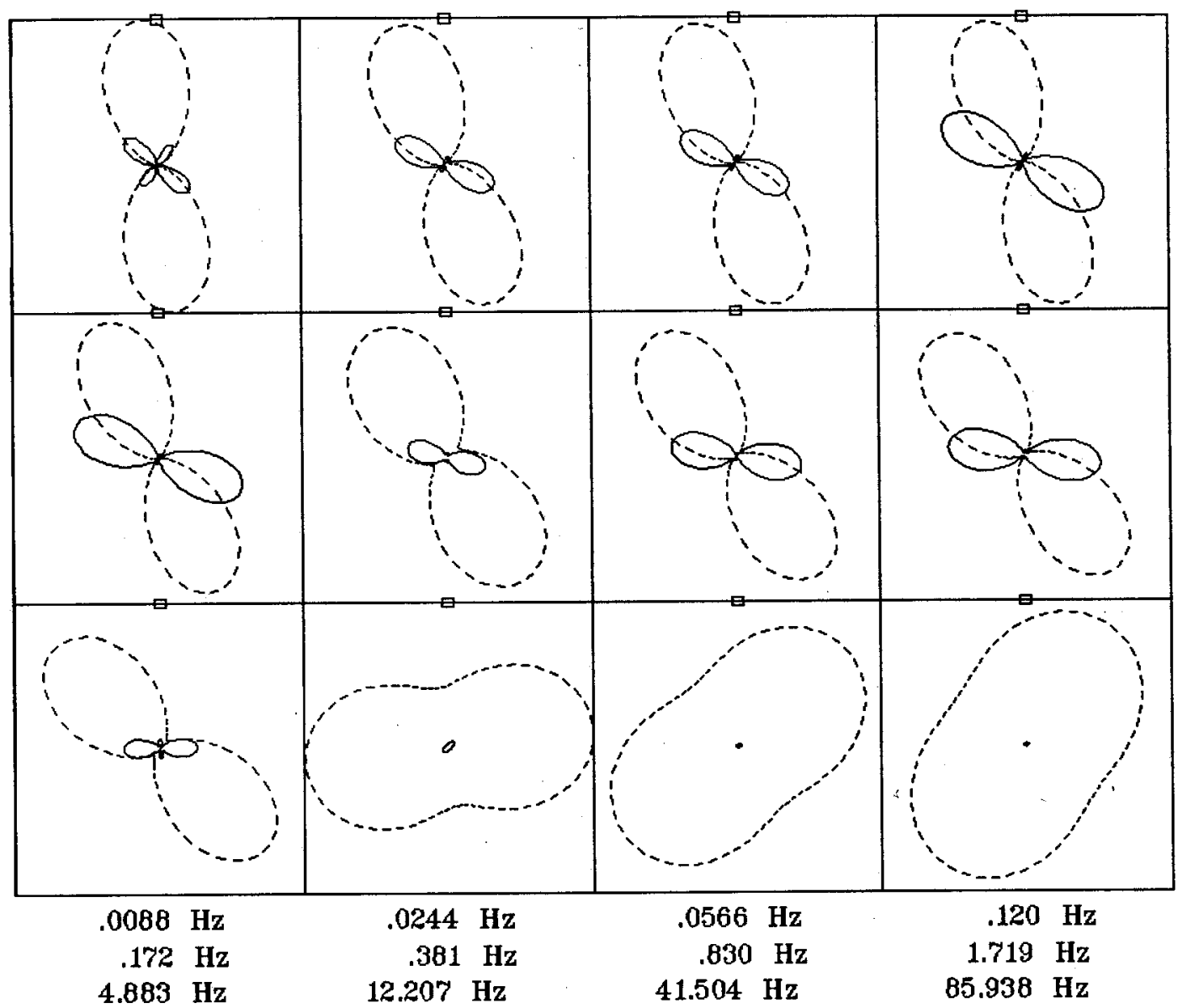

Client: WAter Resources \& DOD Remote: none

Acquired: 07:3 oct 10, 2005 Survey Co:US Geological Survey
Rotation:

Filename: nt52m.avg

Channels: Ch1 Ch2 Ch3 Ch4 Ch5 Ch3 Ch4 Plotted: 15:10 Feb 08, 2006

< EMI - ElectroMagnetic Instruments > 
Station 52

TIPPER MAGNITUDE

Shoshone Peak

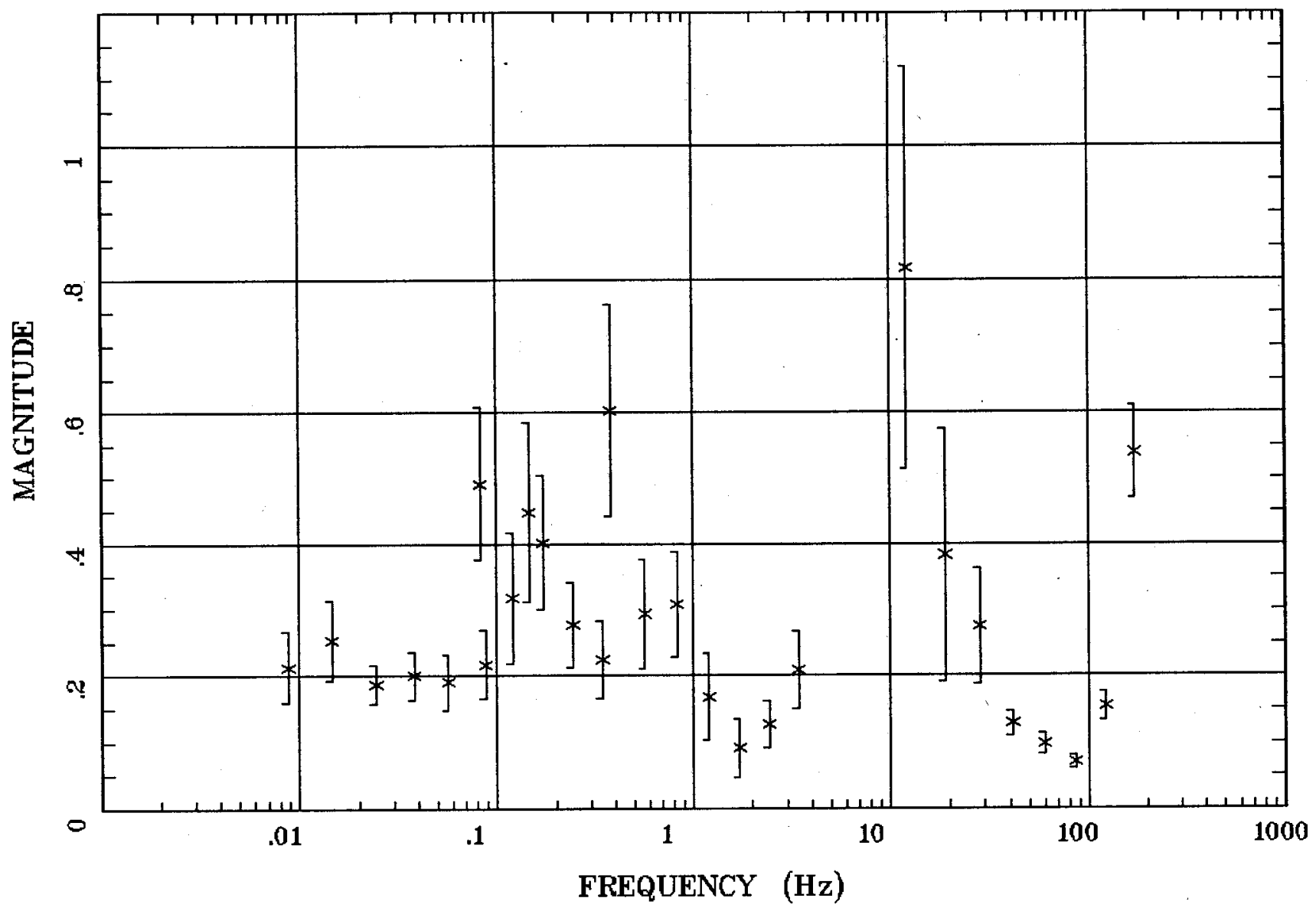

Client: WAter Resources \& DOD Remote: none

Acquired: 07:3 oct 10, 2005 Survey Co:US Geological Survey
Rotation:

Filename: nt52m.avg Channels: Ch1 Ch2 Ch3 Ch4 Ch5 Ch3 Ch4 Plotted: 15:10 Feb 08, 2006

< EMI - ElectroMagnetic Instruments > 


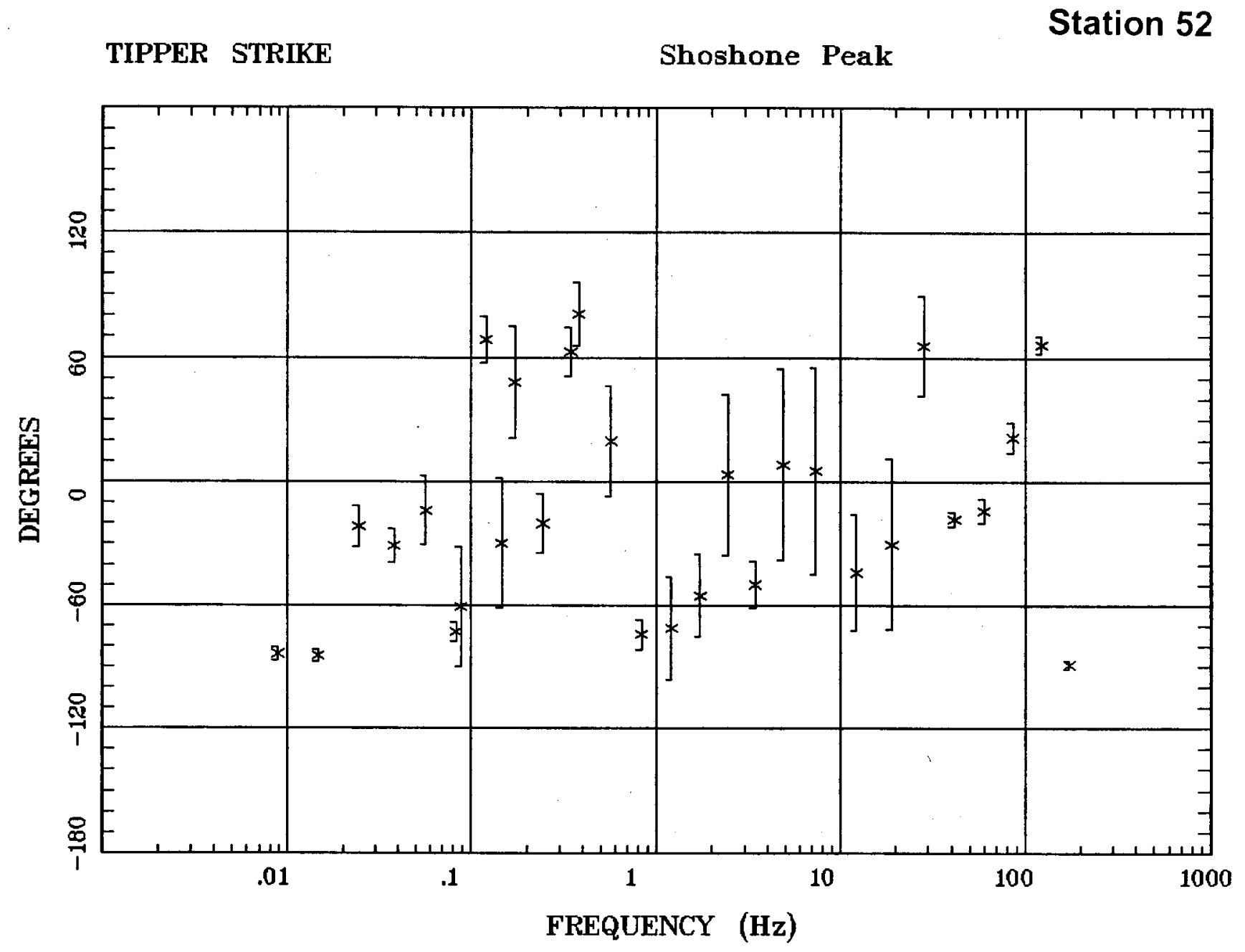

Client: WAter Resources \& DOD Remote: none

Acquired: 07:3 oct 10, 2005 Survey Co:US Geological Survey
Rotation:

Filename: nt52m.avg

Channels: Ch1 Ch2 Ch3 Ch4 Ch5 Ch3 Ch4 Plotted: 15:10 Feb 08, 2006

< EMI - ElectroMagnetic Instruments > 
Station 52

HzHx.x Coh HzHy.o Shoshone Peak

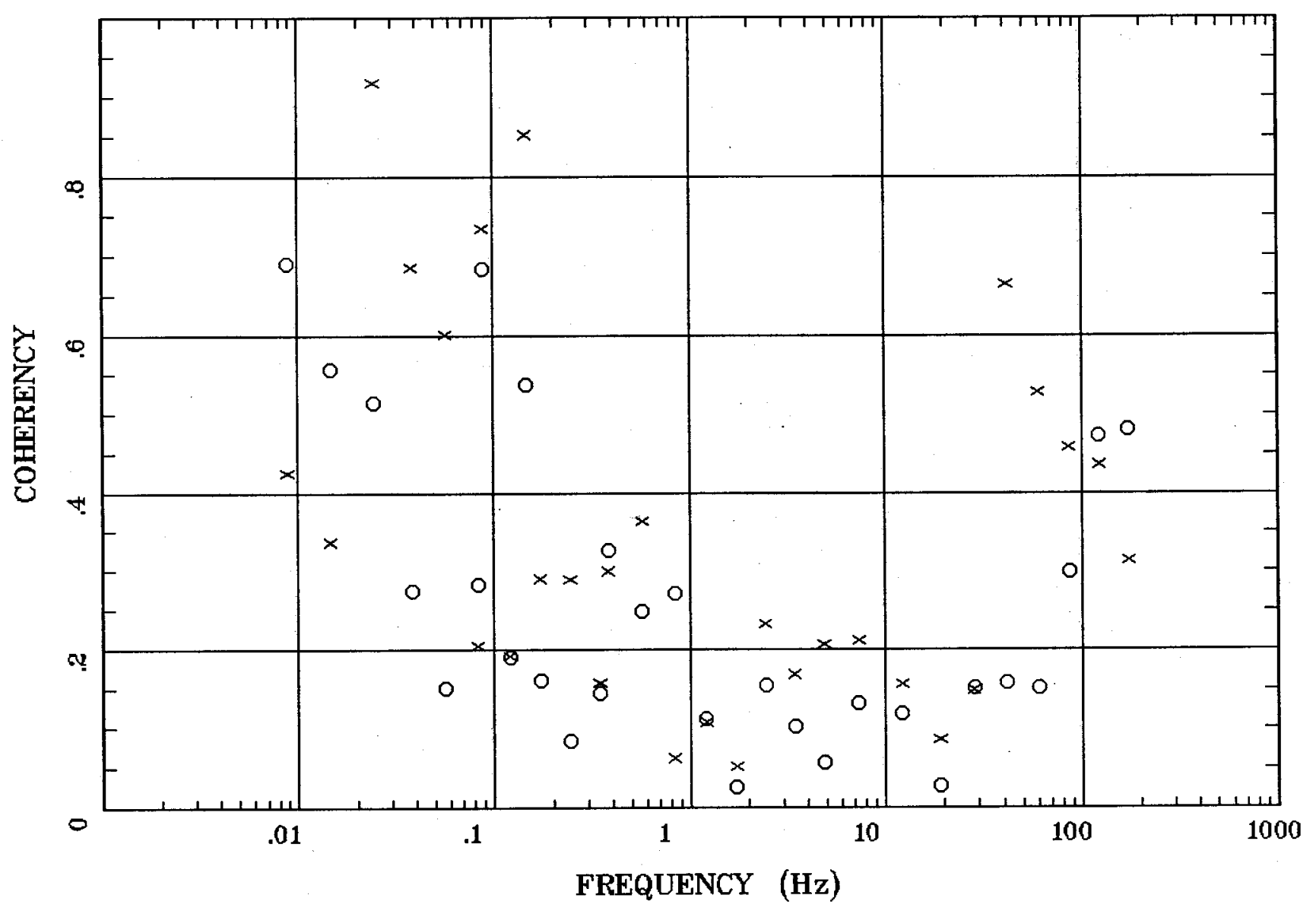

Client: WAter Resources \& DOD Remote: none Acquired: $07: 3$ oct 10, 2005 Survey Co:US Geological Survey
Rotation:

Filename: nt5:m.avg Channels: Ch1 Ch2 Ch3 Ch4 Ch5 Ch3 Ch4 Plotted: 15:10 Feb 08, 2006

< EMI - ElectroMagnetic Instruments > 


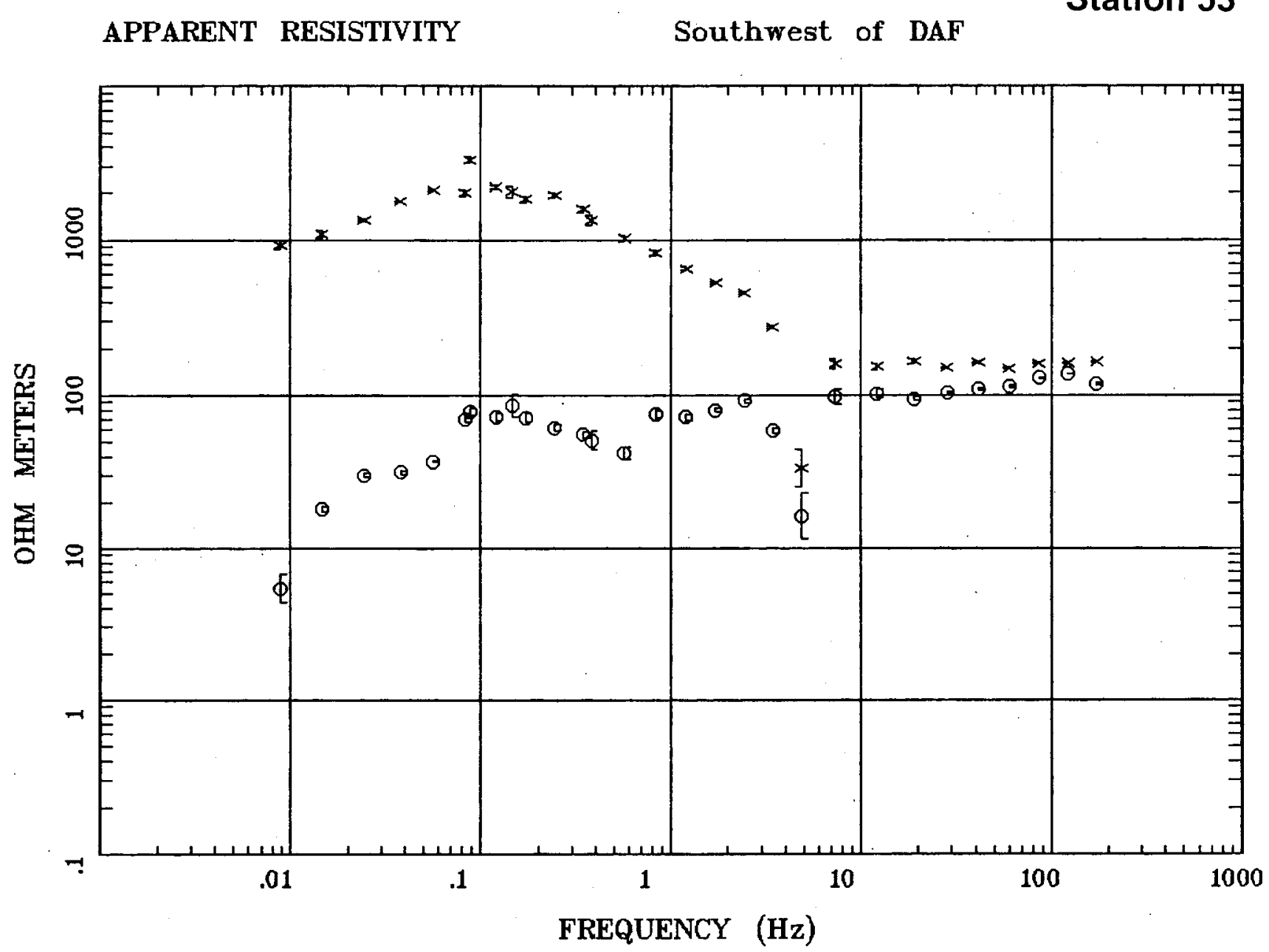

Client: WAter Resources \& DOD Remote: none

Acquired: 06:1 oct 11, 2005 Survey Co:US Geological Survey
Rotation:

Filename: nt53n.avg Channels: Ch1 Ch2 Ch3 Ch4 Ch5 Ch3 Ch4 Plotted: 09:26 Feb 02, 2006

< EMI - ElectroMagnetic Instruments > 
IMPEDANCE PHASE

Southwest of DAF

Station 53

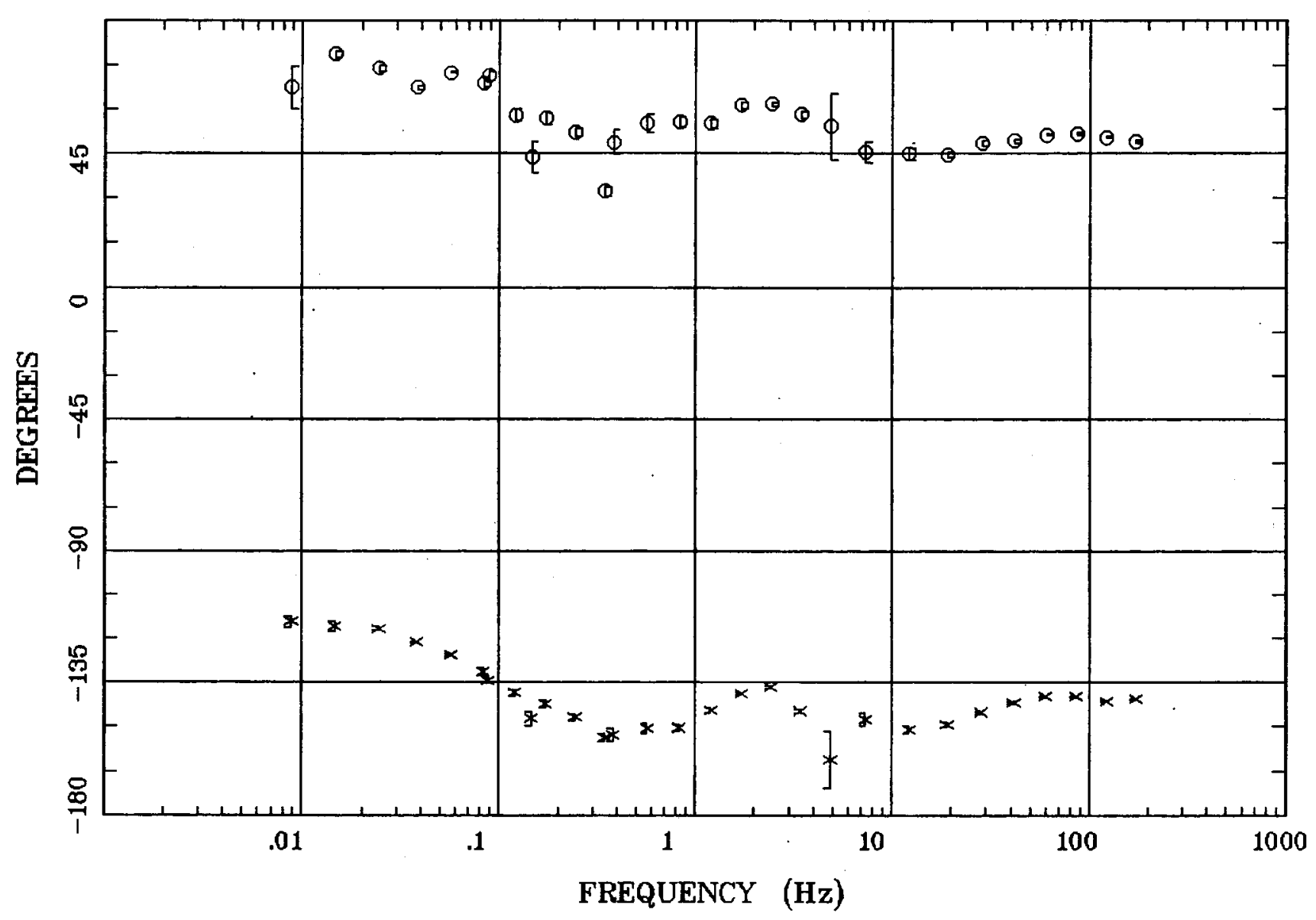

Client: WAter Resources \& DOD Remote: none

Acquired: 06:1 oct 11, 2005 Survey Co:US Geological Survey
Rotation:

Filename: nt53n.avg

Channels: Ch1 Ch2 Ch3 Ch4 Ch5 Ch3 Ch4 Plotted: 09:26 Feb 02, 2006

< EMI - ElectroMagnetic Instruments > 


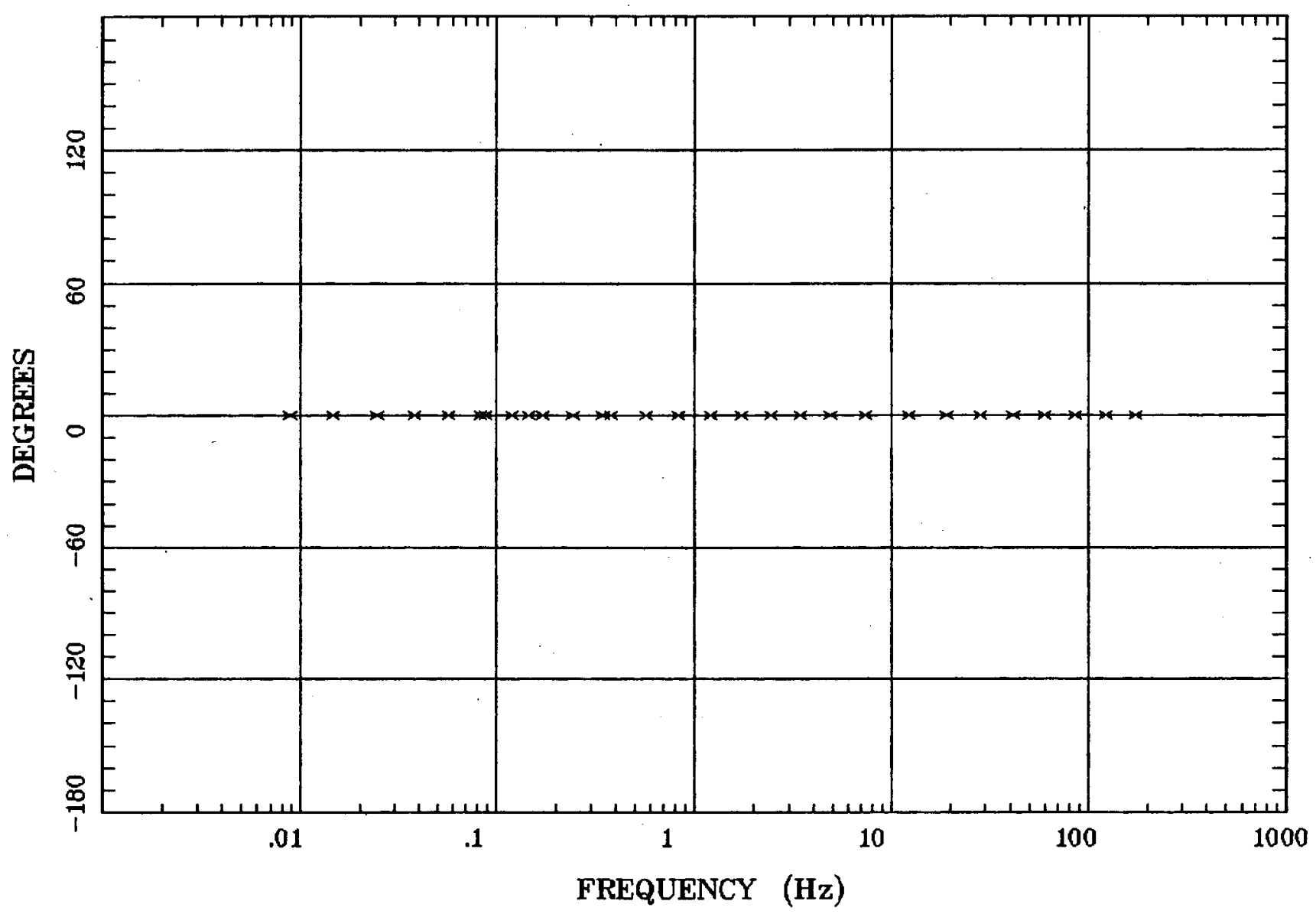

Client: WAter Resources \& DOD Remote: none Acquired: 06:1 Oct 11, 2005 Survey Co:US Geological Survey
Rotation:

Filename: nt53n.avg Channels: Ch1 Ch2 Ch3 Ch4 Ch5 Ch3 Ch4 Plotted: 09:26 Feb 02, 2006

< EMI - ElectroMagnetic Instruments > 


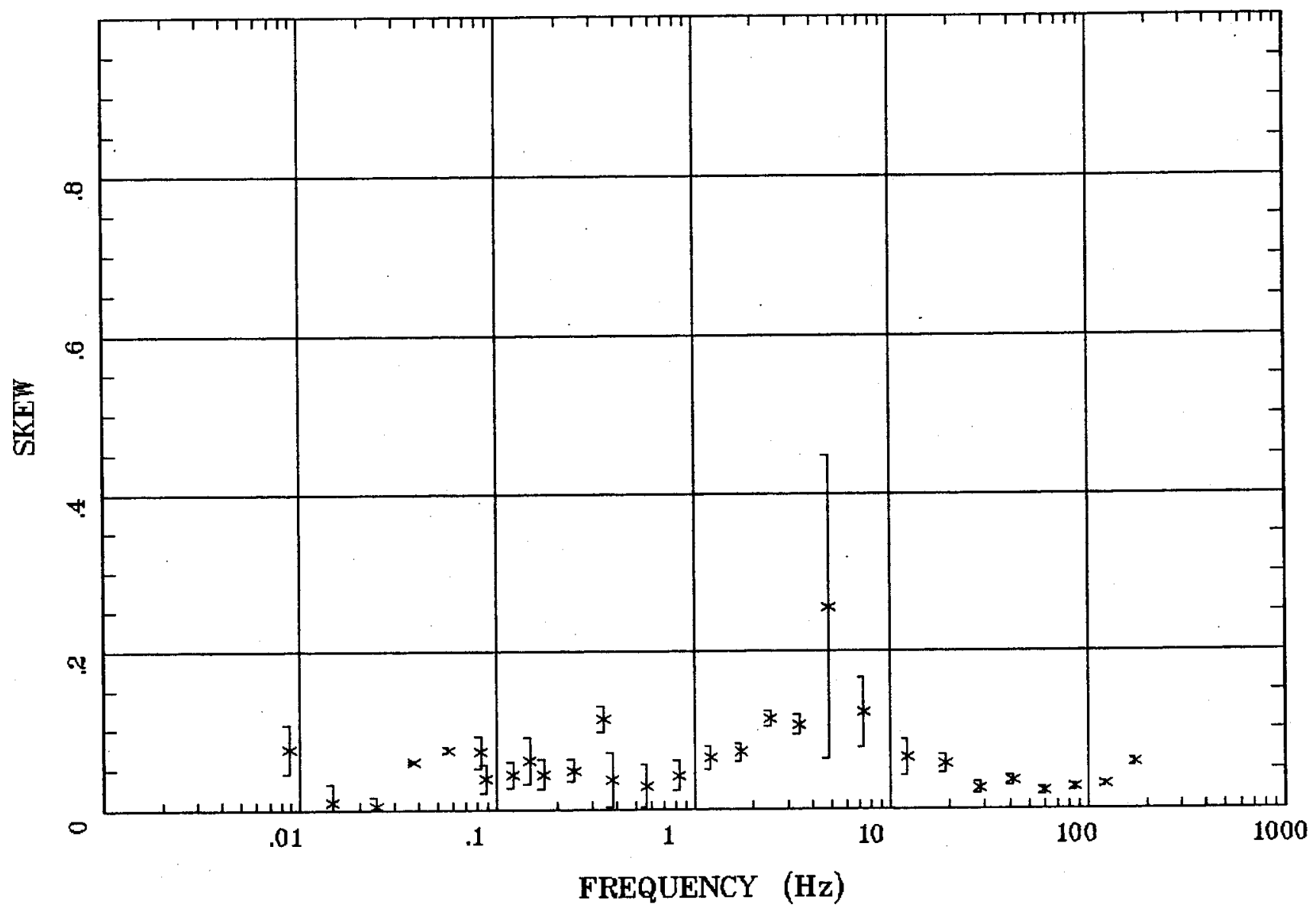

Client: WAter Resources \& DOD Remote: none Acquired: 06:1 Oct 11, 2005 Survey Co:US Geological Survey
Rotation:

Filename: nt53n.avg Channels: Ch1 Ch2 Ch3 Ch4 Ch5 Ch3 Ch4 Plotted: 09:26 Feb 02, 2006

< EMI - ElectroMagnetic Instruments > 


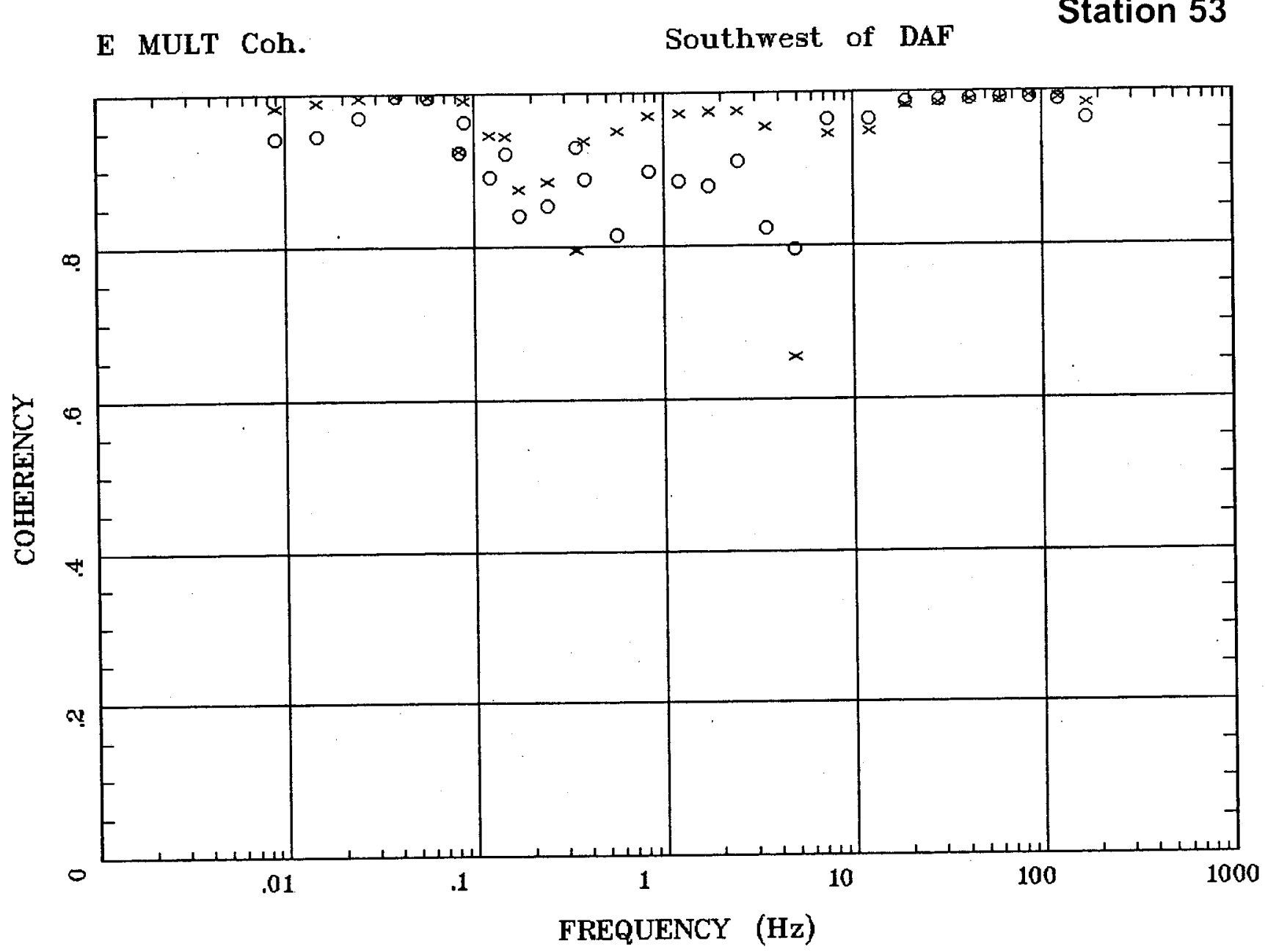

Client: WAter Resources \& DOD Remote: none

Acquired: 06:1 oct 11, 2005 Survey Co:US Geological Survey

Rotation:

Filename: nt53n.avg

Channels: Ch1 Ch2 Ch3 Ch4 Ch5 Ch3 Ch4

Plotted: 09:26 Feb 02, 2006

< EMI - ElectroMagnetic Instruments > 


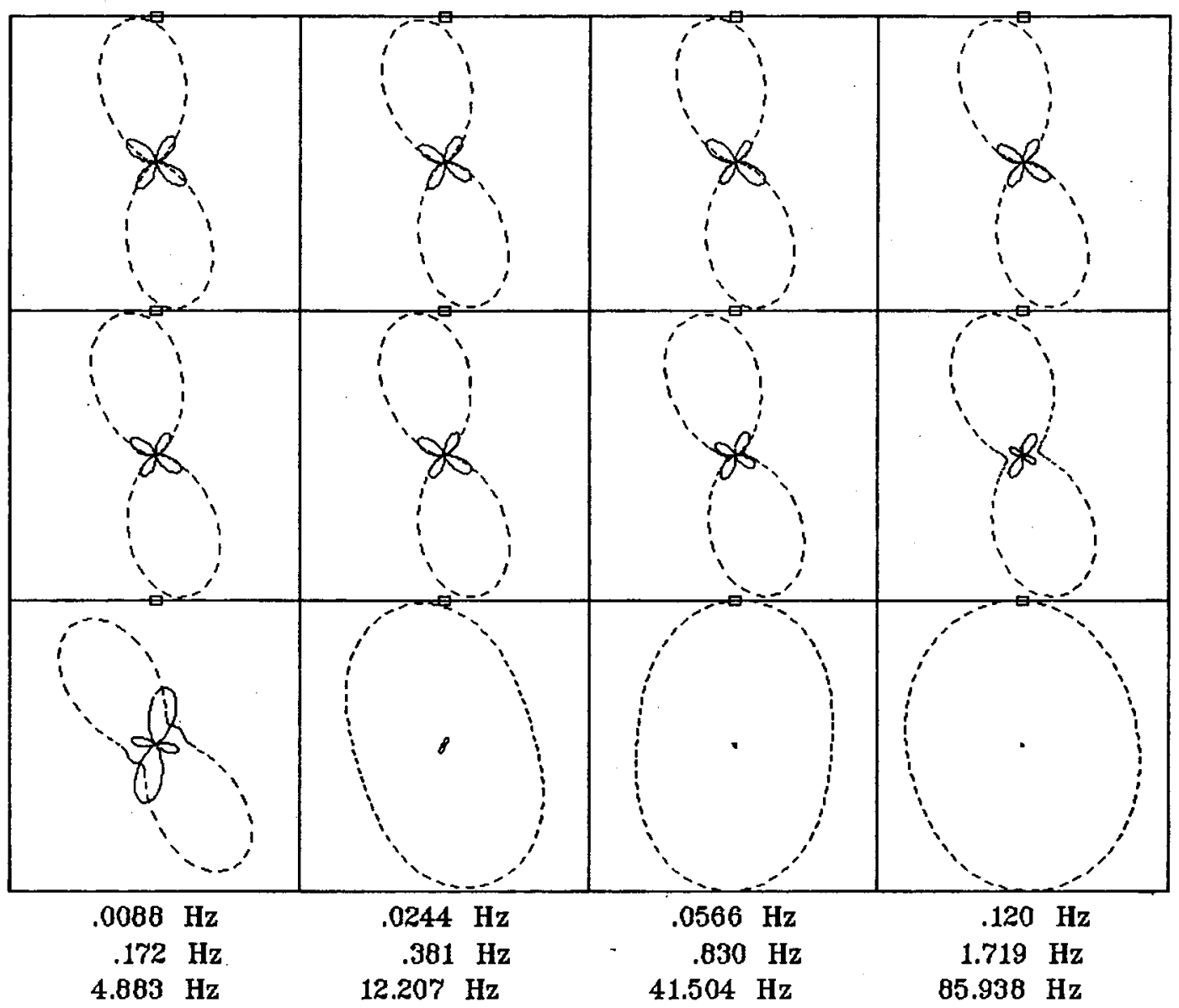

Client: WAter Resources \& DOD Remote: none

Acquired: 06:1 Oct 11, 2005

Survey Co:US Geological Survey
Rotation:

Filename: nt53n.evg

Channels: Ch1 Ch2 Ch3 Ch4 Ch5 Ch3 Ch4

Plotted: 09:26 Feb 02, 2006

< EMI - ElectroMagnetic Instruments > 
TIPPER MAGNITUDE

Southwest of DAF

Station 53

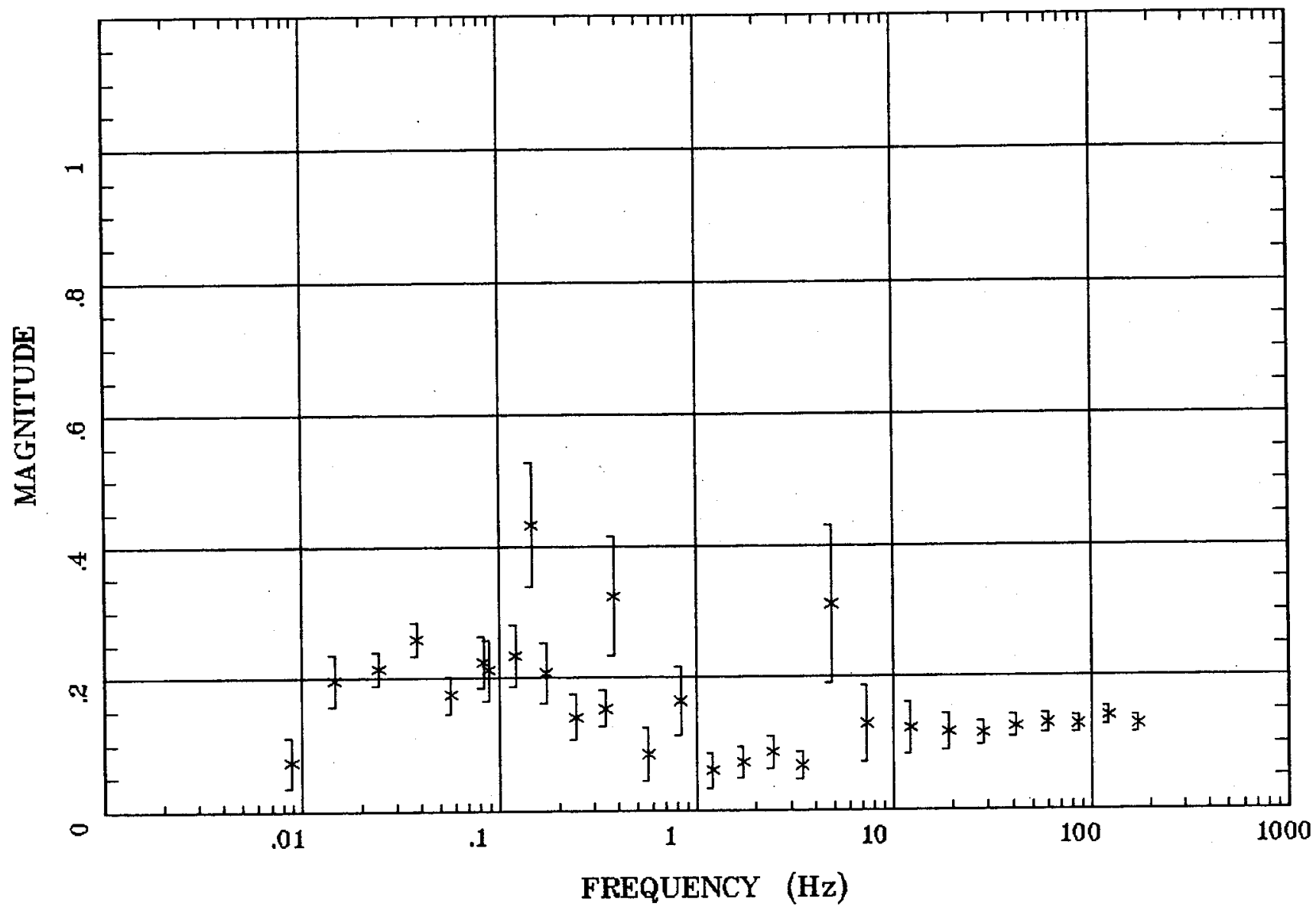

Client: WAter Resources \& DOD Remote: none Acquired: 06:1 oct 11, 2005 Survey Co:US Geological Survey
Rotation:

Filename: nt53n.avg

Channels: Ch1 Ch2 Ch3 Ch4 Ch5 Ch3 ch4

Plotted: 09:26 Feb 02, 2006

< EMI - ElectroMagnetic Instruments > 


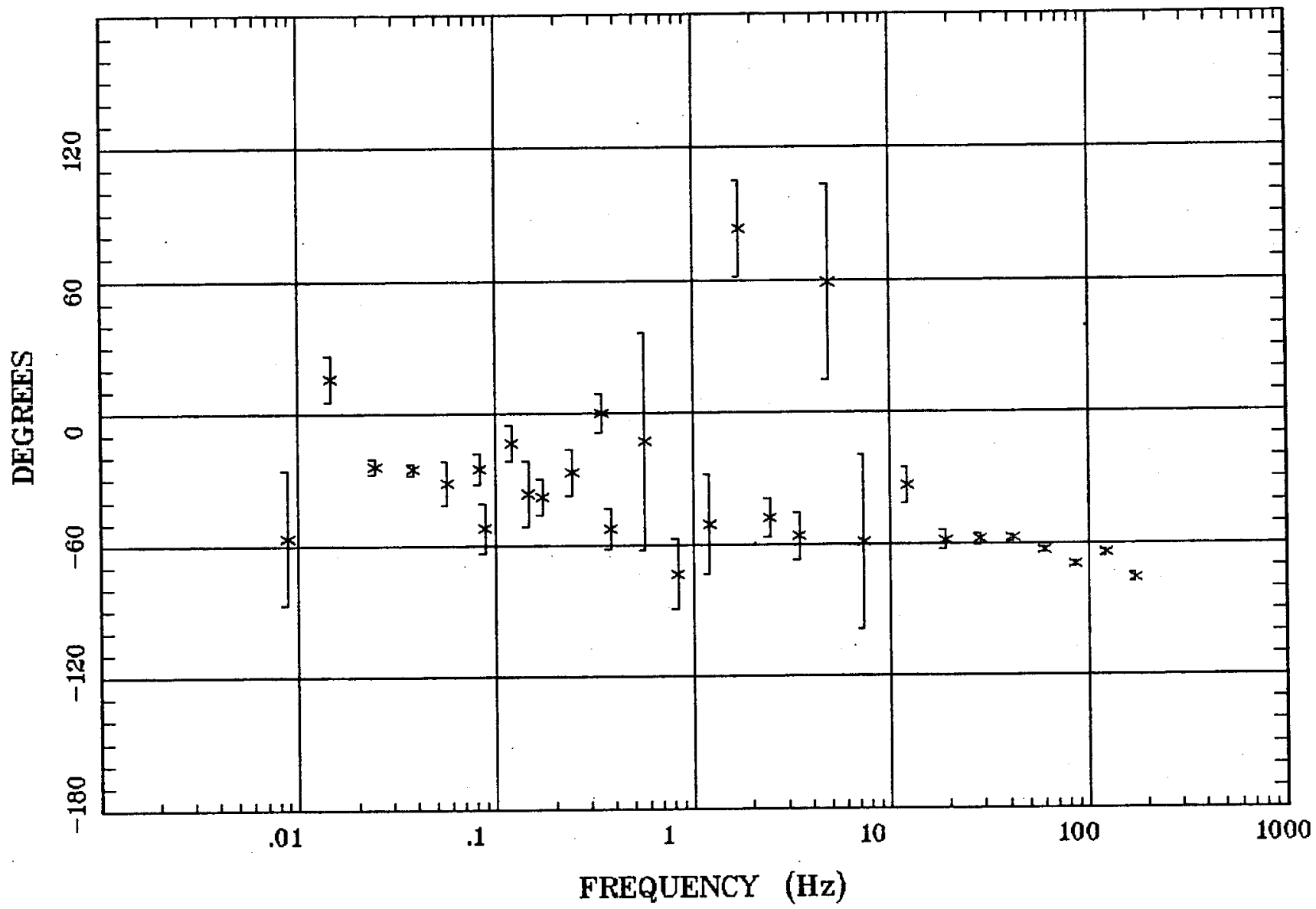

Client: WAter Resources \& DOD Remote: none Acquired: 06:1 oct 11, 2005 Survey Co:US Geological Survey
Rotation:

Filename: nt53n.avg Channels: Ch1 Ch2 Ch3 Ch4 Ch5 Ch3 Ch4 Plotted: 09:26 Feb 02, 2006

< EMI - ElectroMagnetic Instruments > 


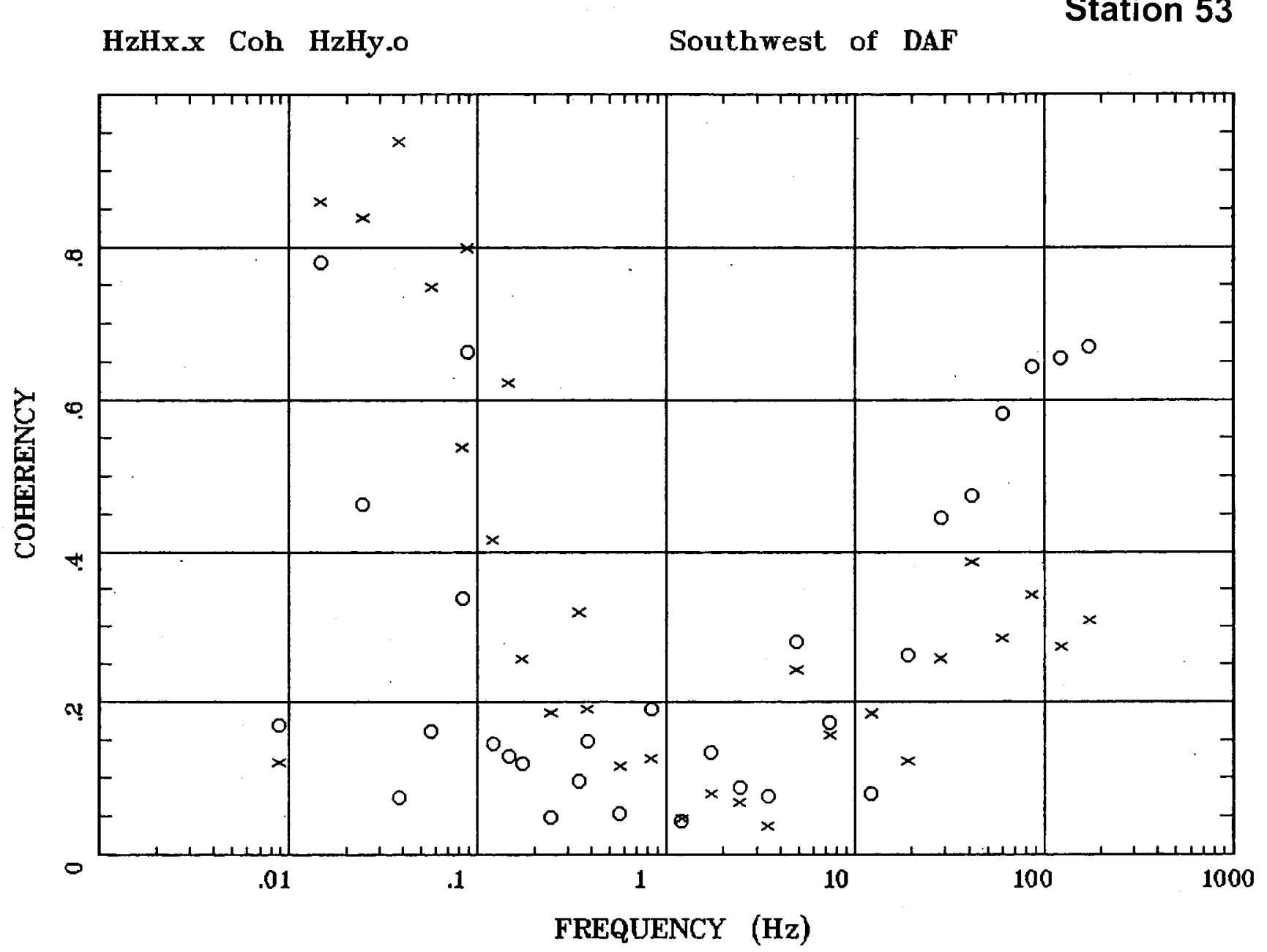

Client: WAter Resources \& DOD Remote: none

Acquired: 06:1 Oct 11, 2005

Survey Co:US Geological Survey
Rotation:

Filename: nt53n.avg Channels: Ch1 Ch2 Ch3 Ch4 Ch5 Ch3 Ch4 Plotted: 09:26 Feb 02, 2006

< EMI - ElectroMagnetic Instruments. > 


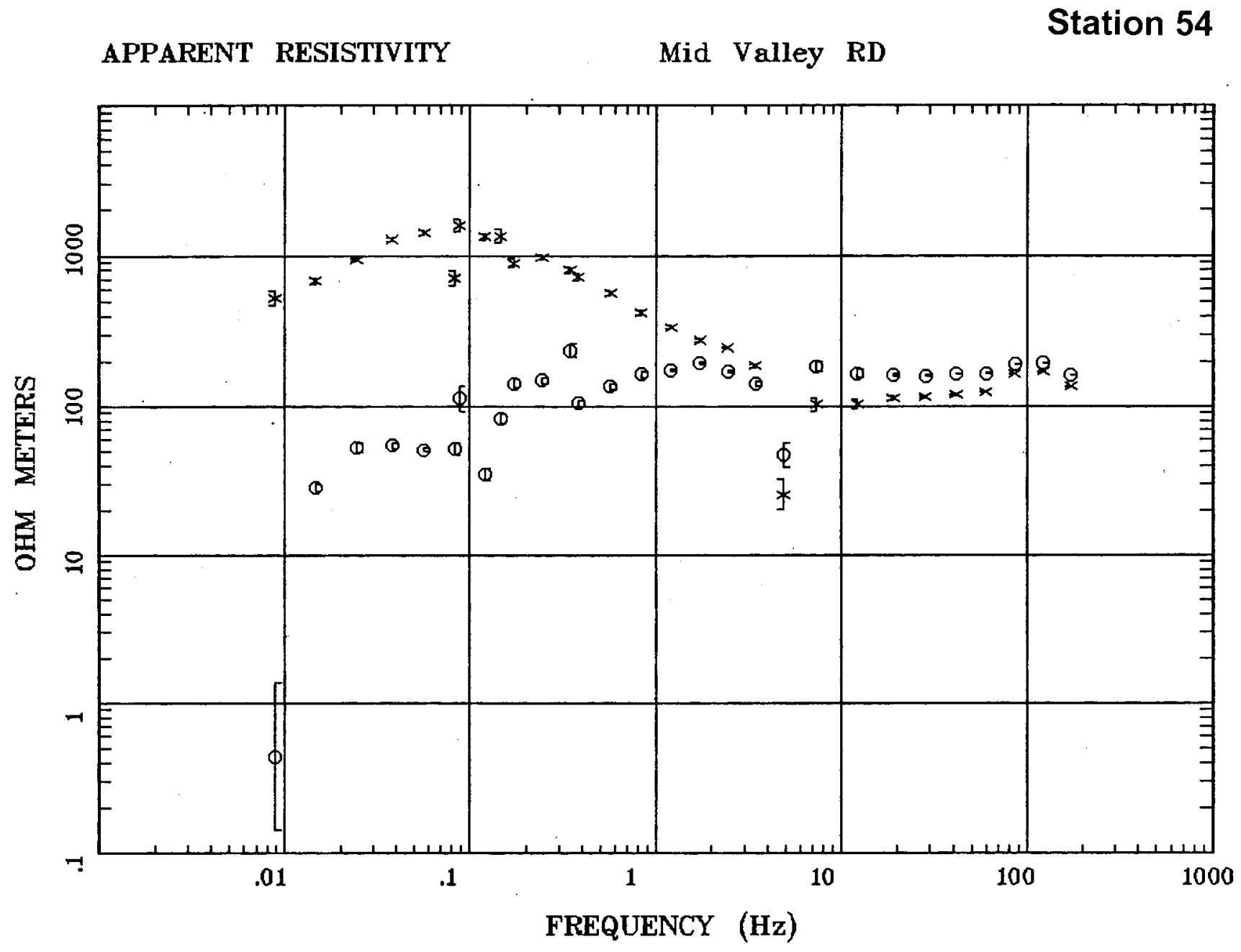

Client: WAter Resources \& DOD Remote: none

Acquired: $04: 5$ oct 12, 2005 Survey Co:US Geological Survey
Rotation:

Filename: nt54n.avg

Channels: Ch1 Ch2 Ch3 Ch4 Ch5 Ch3 Ch4 Plotted: 09:56 Feb 02, 2006

< EMI - ElectroMagnetic Instruments > 
IMPEDANCE PHASE

Mid Valley RD

Station 54

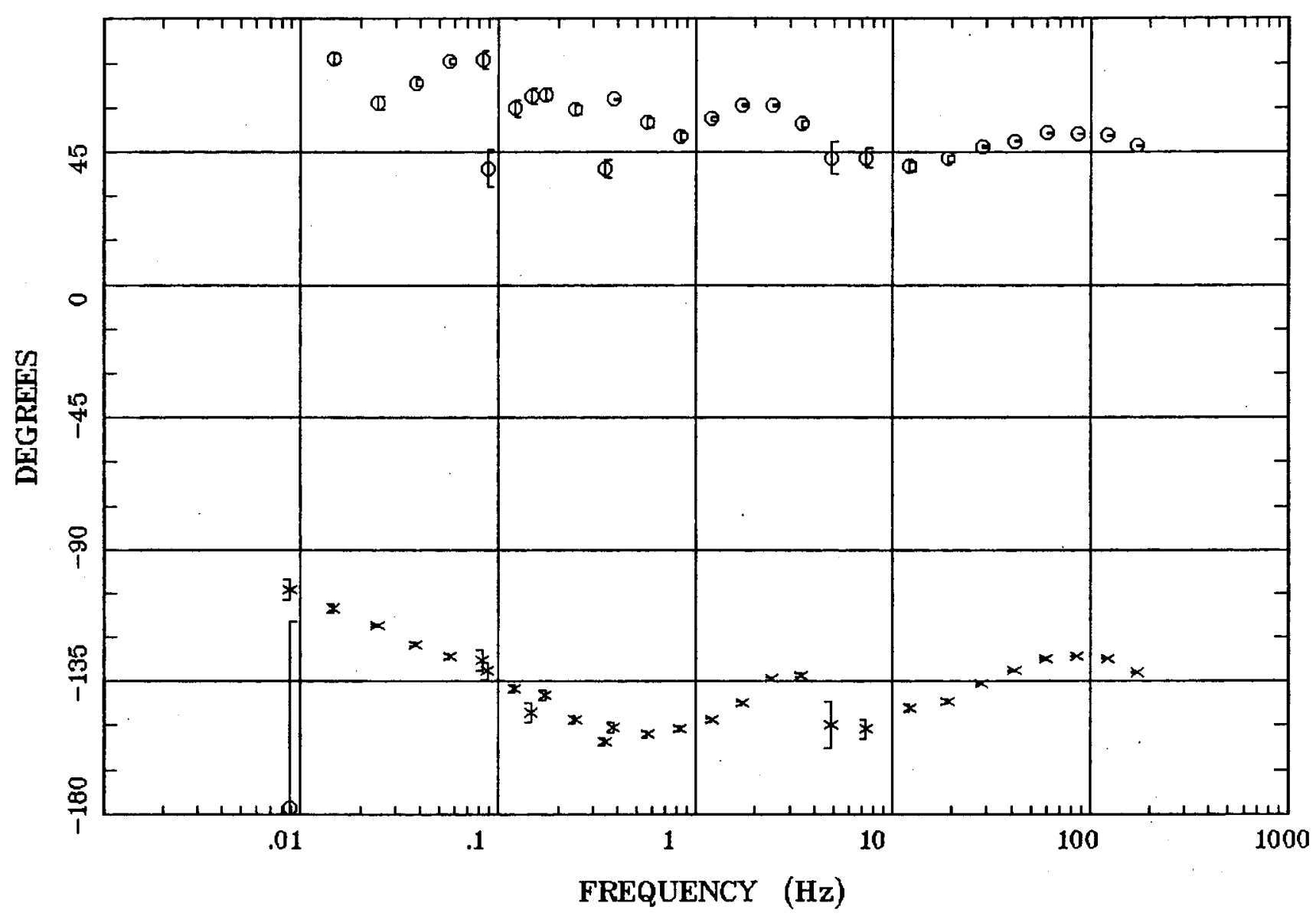

Client: WAter Resources \& DOD Remote: none

Acquired: 04:5 Oct 12, 2005 Survey Ca:US Geological Survey
Ratation:

Filename: nt54n.avg

Channels: Ch1 Ch2 Ch3 Ch4 Ch5 Ch3 Ch4 Plotted: 09:56 Feb 02, 2006

< EMI - ElectroMagnetic Instruments > 
ROTATION ANGLE

Mid Valley RD

Station 54

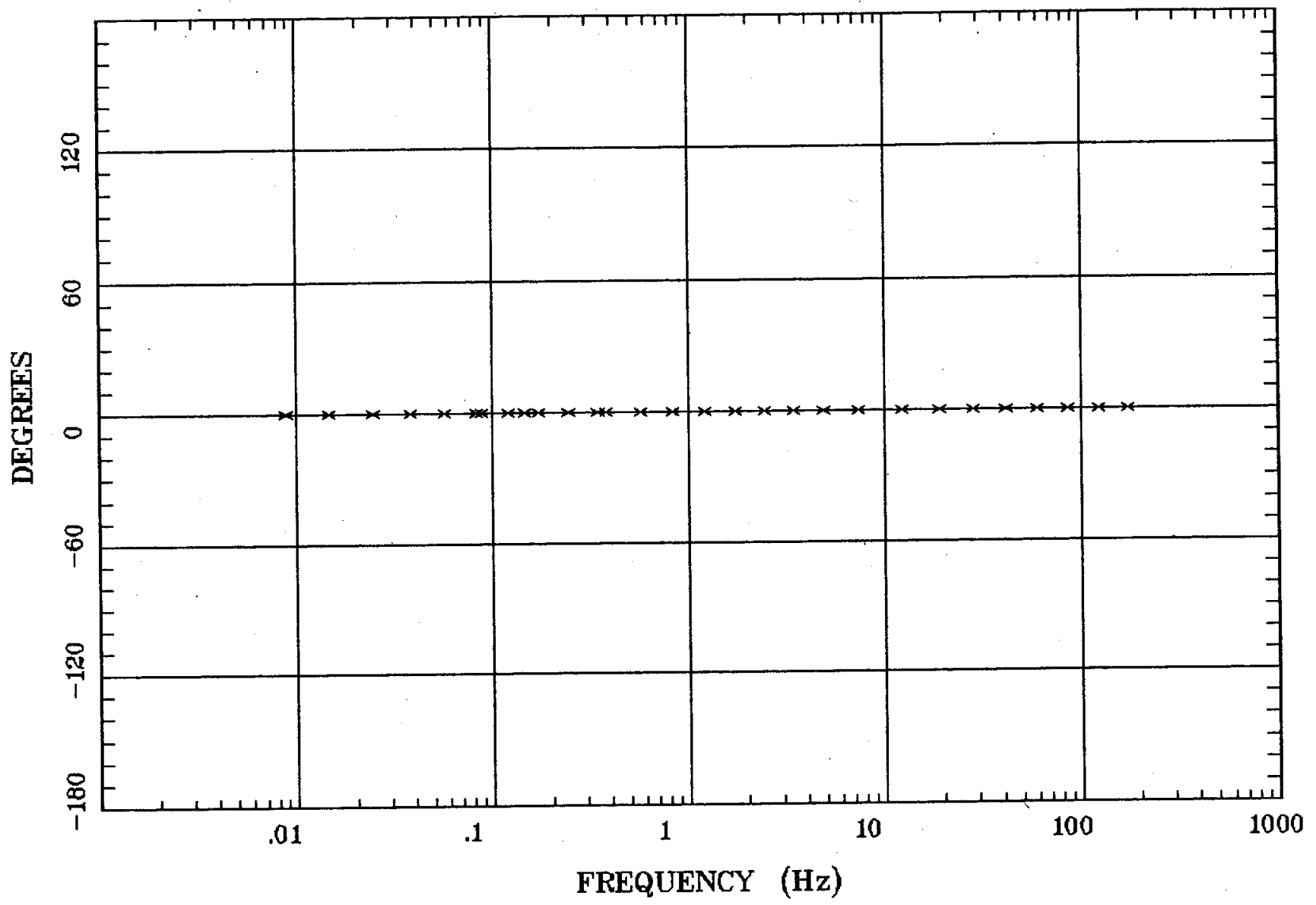

Client: FAter Resources \& DOD

Rotation:

Remote: none

Acquired: 04:5 oct 12, 2005

Survey Co:US Geological Survey

Filename: nt54n.avg

Channels: Ch1 Ch2 Ch3 Ch4 Ch5 Ch3 Ch4

Plotted: 09:56 Feb 02, 2006

< EMI - ElectroMagnetic Instruments > 


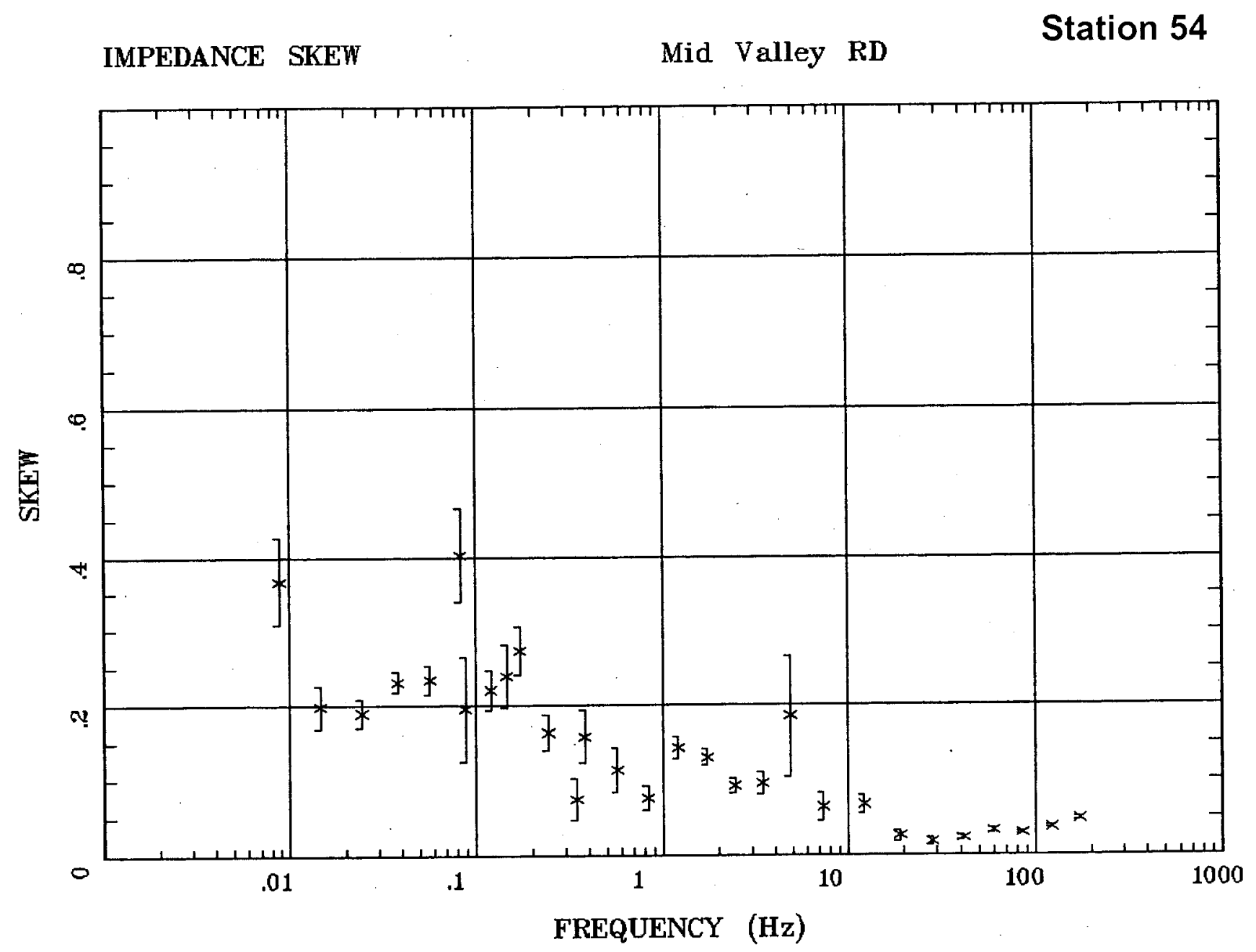

Client: WAter Resources \& DOD

Rotation:

Remote: none

Acquired: 04:5 oct 12, 2005

Survey Co:US Geological Survey

Filename: nt54n.avg

Channels: Ch1 Ch2 Ch3 Ch4 Ch5 Ch3 Ch4

Plotted: 09:56 Feb 02, 2006

< EMI - ElectroMagnetic Instruments > 
E MULT Coh.

Mid Valley RD

Station 54

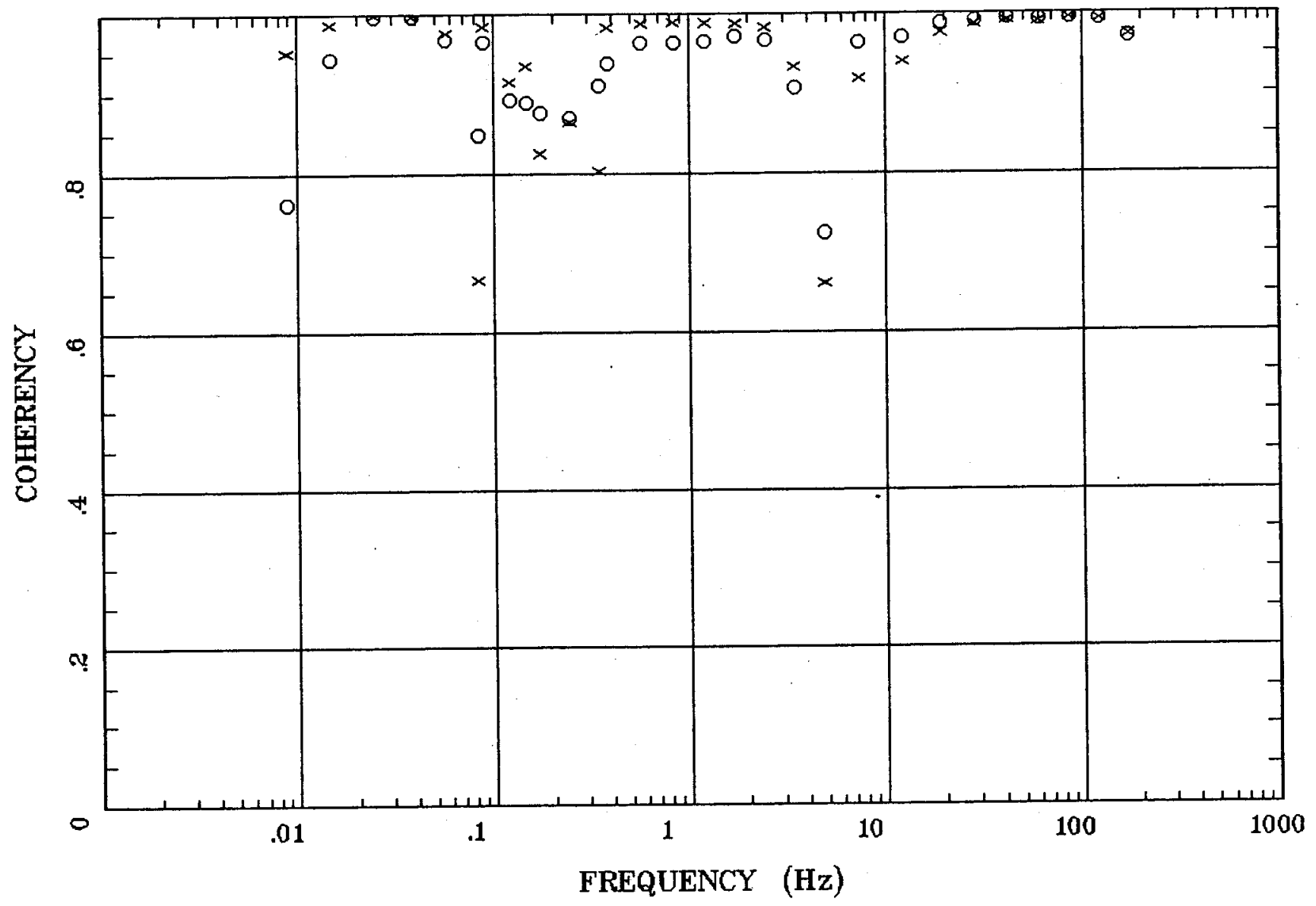

Client: WAter Resources \& DOD

Rotation:

Remote: none

Acquired: 04:5 oct 12, 2005

Survey Co:US Geological Survey

Filename: nt54n.avg

Channels: Ch1 Ch2 Ch3 Ch4 Ch5 Ch3 Ch4

Plotted: 09:56 Feb 02, 2006

< EMI - ElectroMagnetic Instruments > 


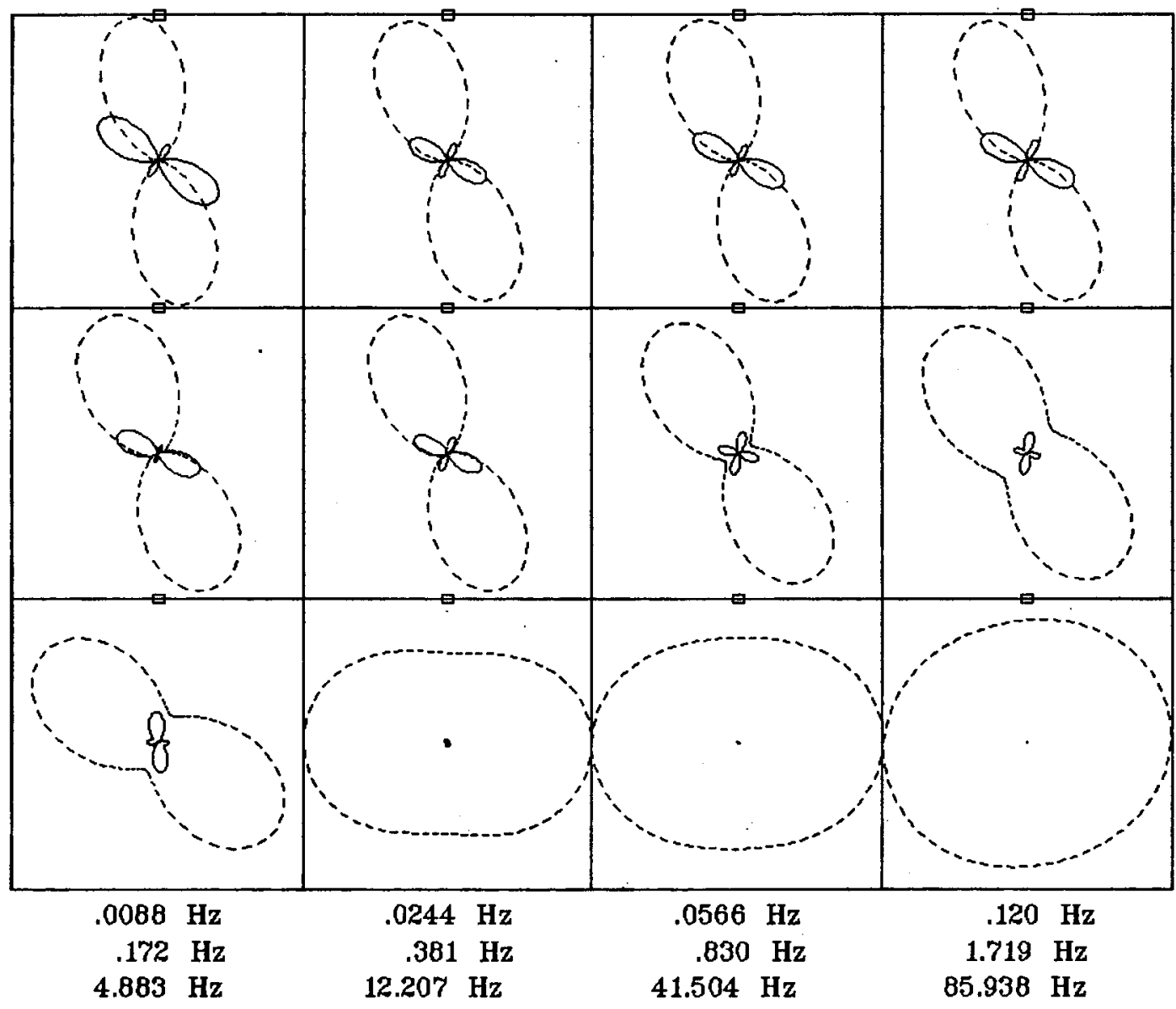

Client: WAter Resources \& DOD Remote: none Acquired: 04:5 oct 12, 2005 Survey Co:US Geological Survey
Rotation:

Filename: nt54n.avg Channels: Ch1 Ch2 Ch3 Ch4 Ch5 Ch3 Ch4 Plotted: 09:56 Feb 02, 2006

< EMI - ElectroMagnetic Instruments > 


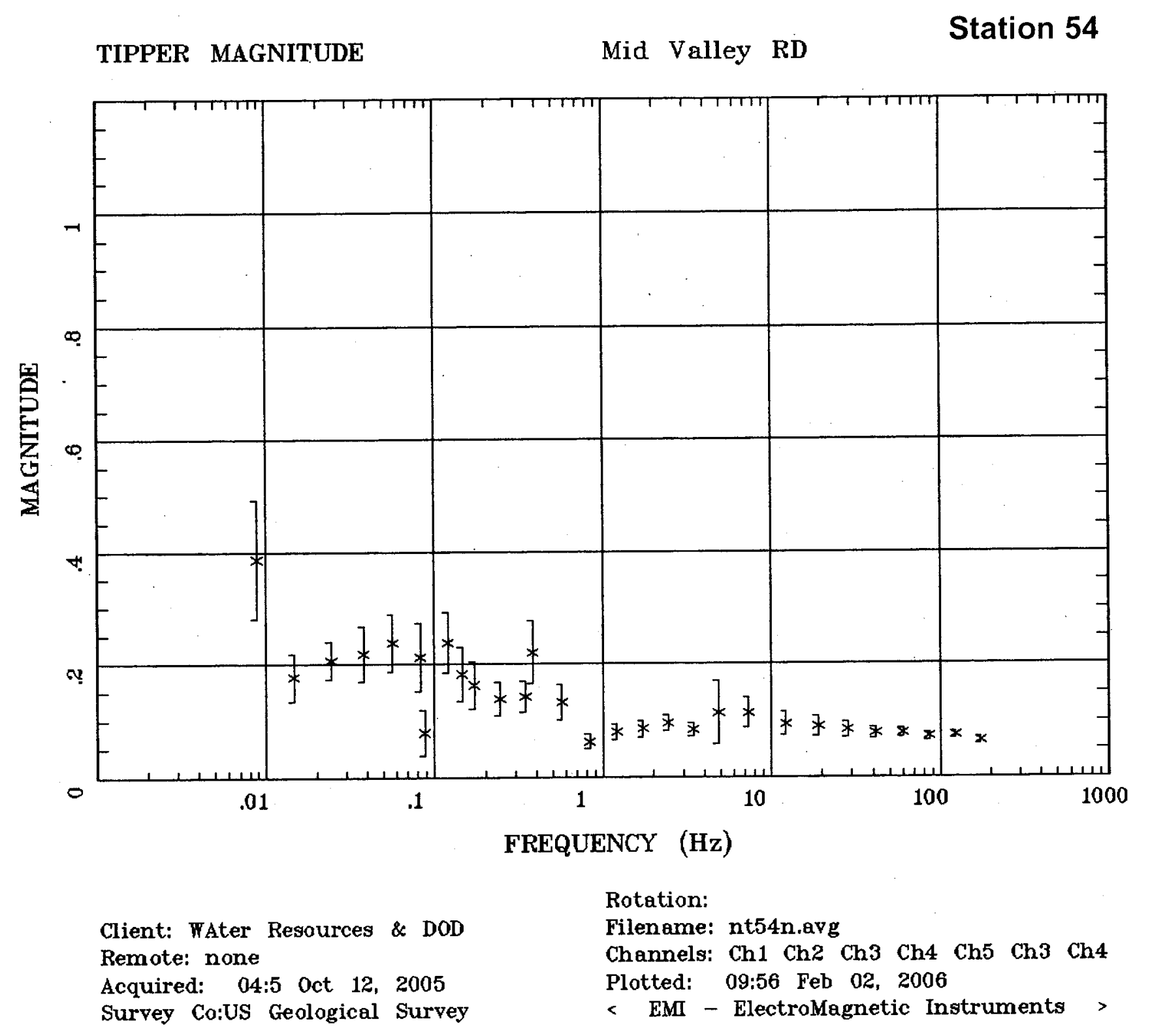




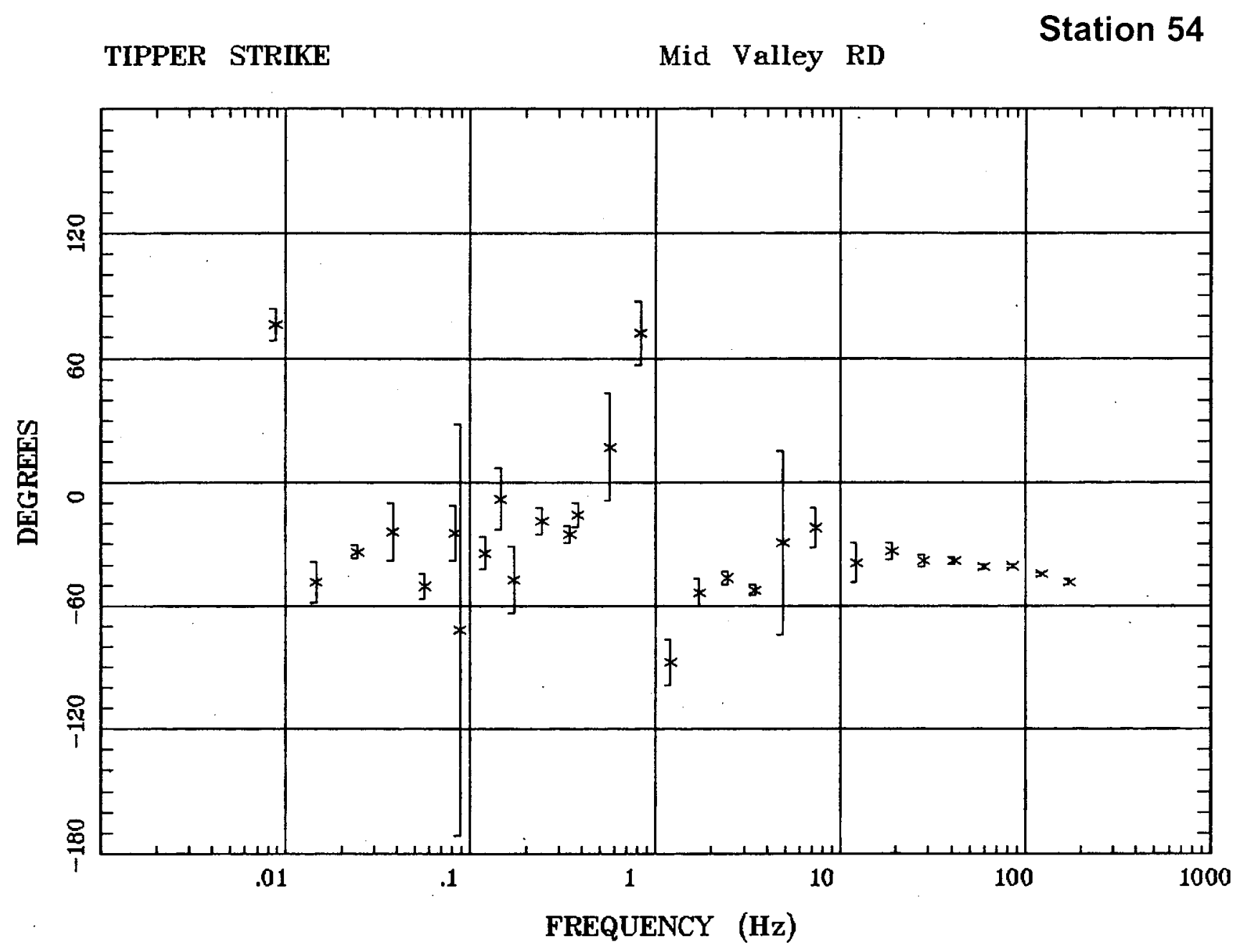

Client: FAter Resources \& DOD Remote: none

Acquired: 04:5 Oct 12, 2005 Survey Co:US Geological Survey
Rotation:

Filename: nt54n.avg Channels: Ch1 Ch2 Ch3 Ch4 Ch5 Ch3 Ch4 Plotted: 09:56 Feb 02, 2006

< EMI - ElectroMagnetic Instruments > 


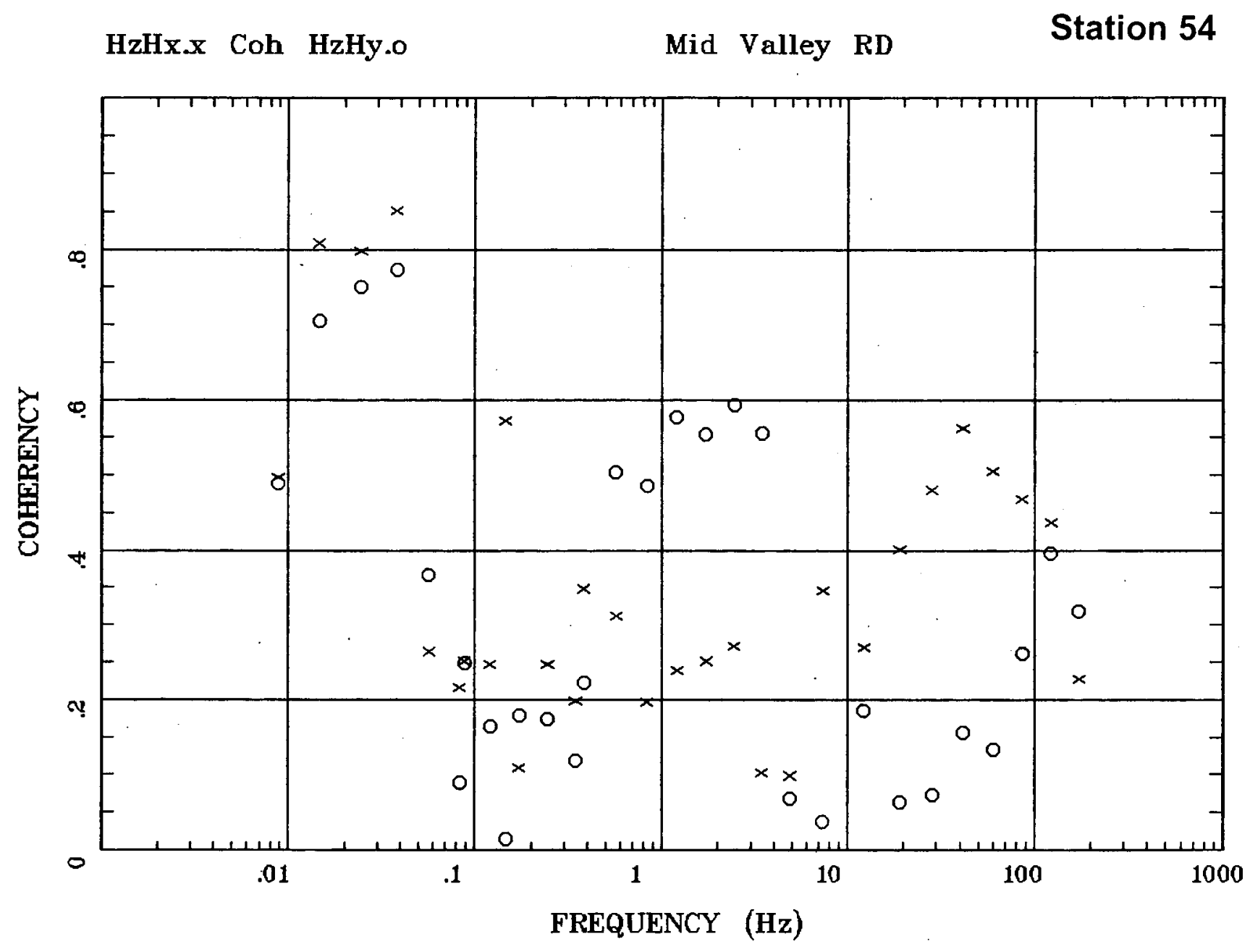

Client: WAter Resources \& DOD

Rotation:

Filename: nt54n.avg

Channels: Ch1 Ch2 Ch3 Ch4 Ch5 Ch3 Ch4

Plotted: 09:56 Feb 02, 2006

Acquired: 04:5 Oct 12, 2005

Survey Co:US Geological Survey

< EMI - ElectroMagnetic Instruments > 


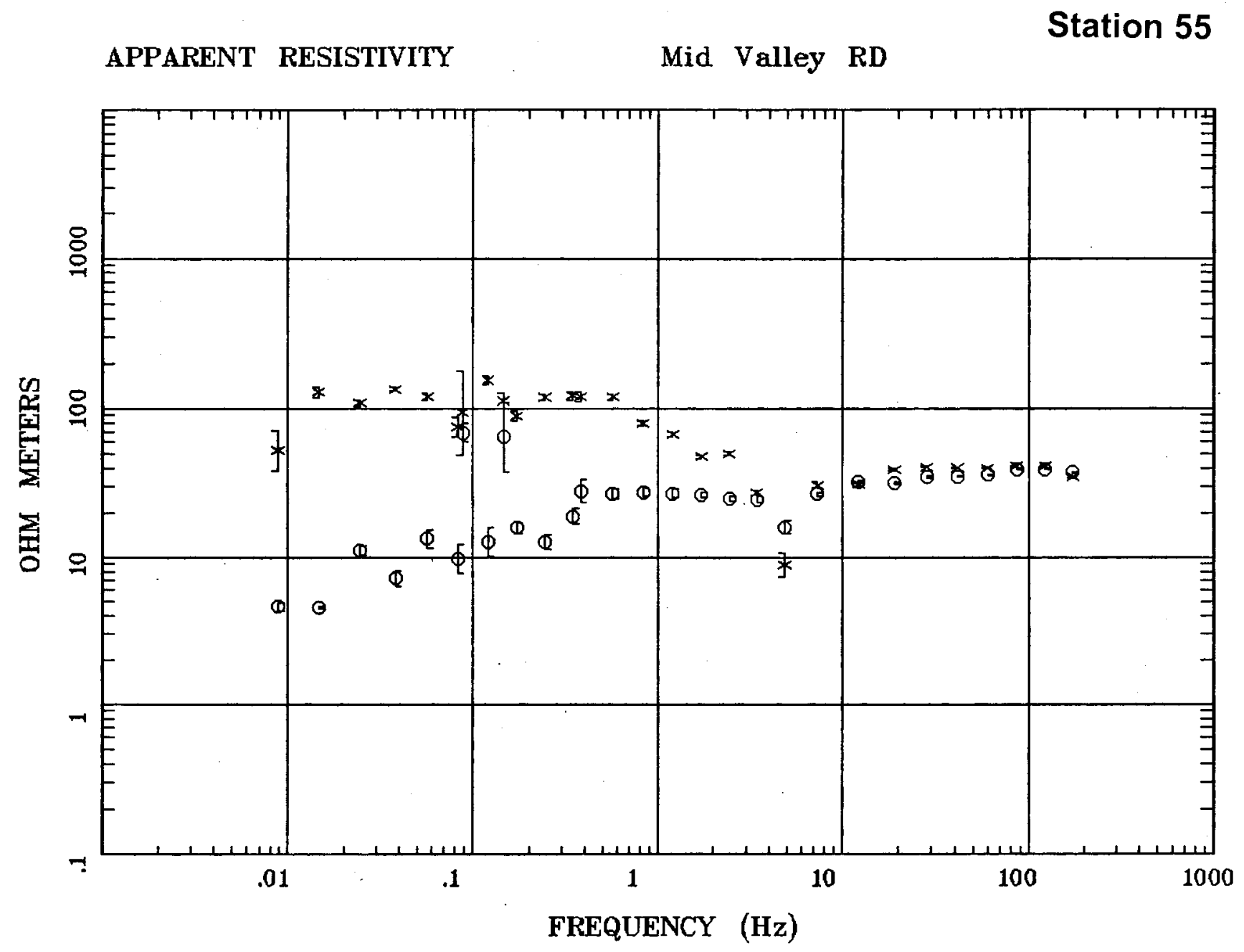

Client: WAter Resources \& DOD Remote: none

Acquired: 06:3 oct 13, 2005

Survey Co:US Geological Survey
Rotation:

Filename: nt55n.avg

Channels: Ch1 Ch2 Ch3 Ch4 Ch5 Ch3 Ch4 Plotted: 09:57 Feb 02, 2006

< EMI - ElectroMagnetic Instruments > 
IMPEDANCE PHASE

Mid Valley RD

Station 55

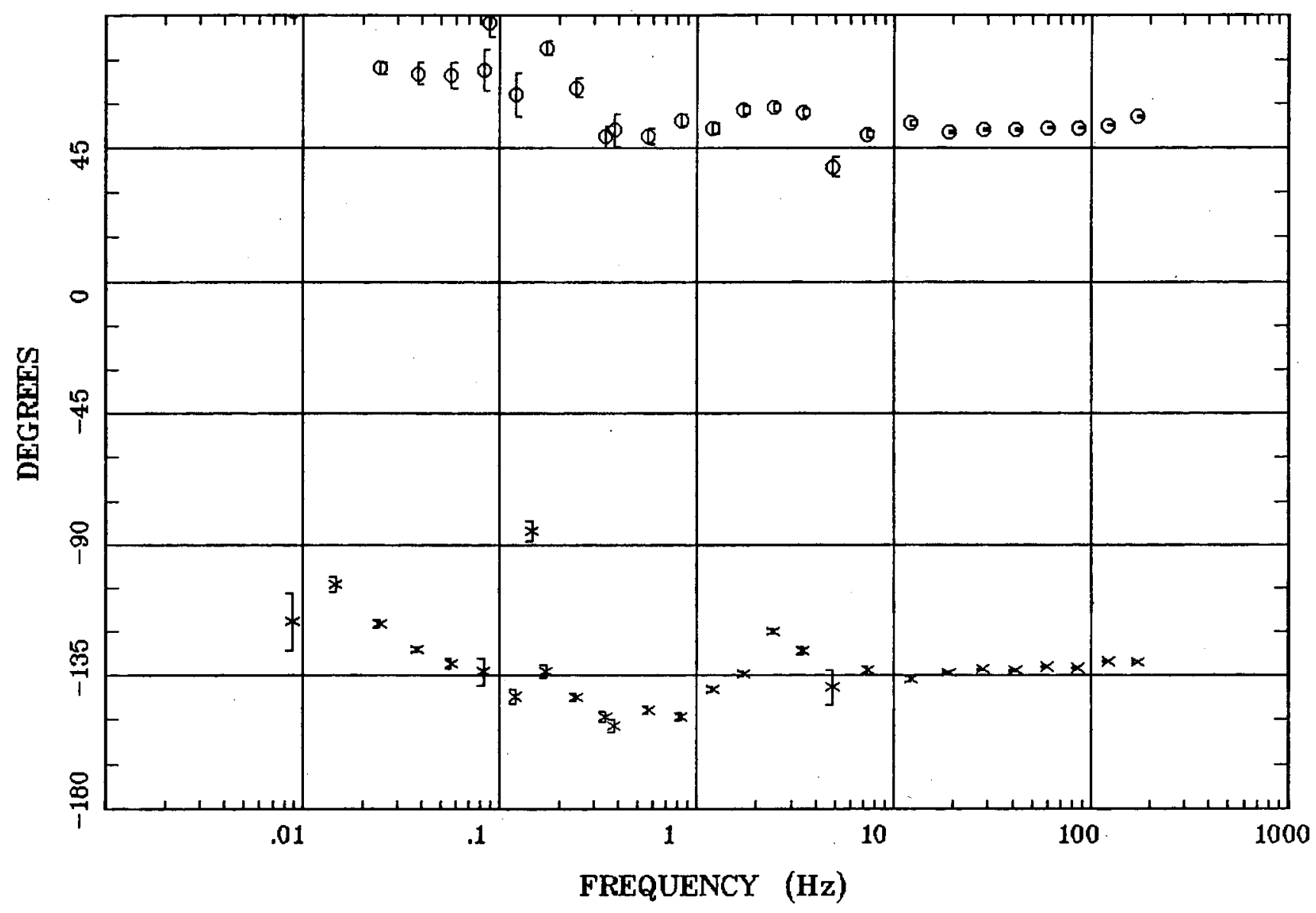

Client: WAter Resources \& DOD Remote: none Acquired: 06:3 Oct 13, 2005 Survey Co:US Geological Survey
Rotation:

Filename: nt55n.avg

Channels: Ch1 Ch2 Ch3 Ch4 Ch5 Ch3 Ch4 Plotted: 09:57 Feb 02, 2006

< EMI - ElectroMagnetic Instruments > 


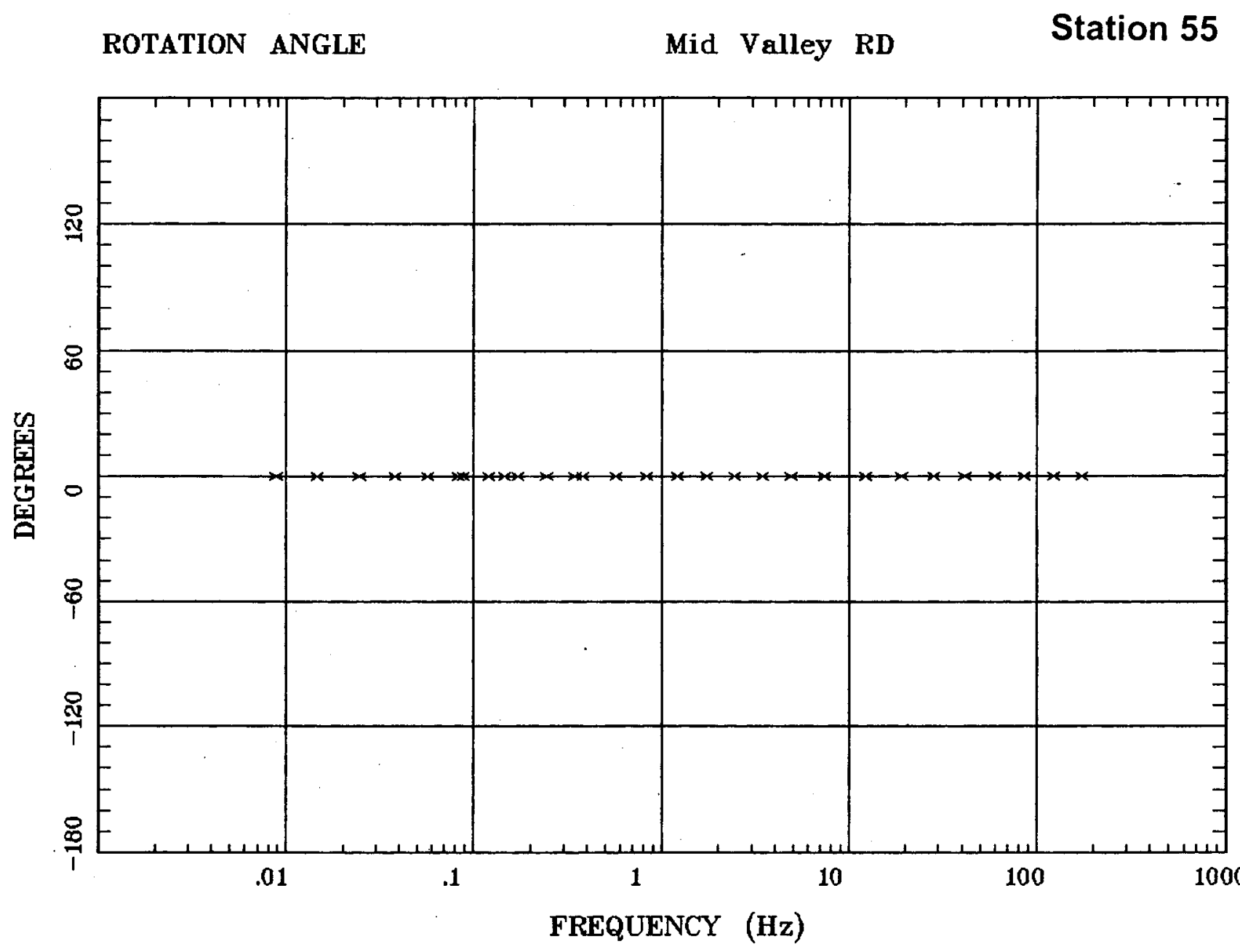

Client: WAter Resources \& DOD

Rotation:

Remote: none

Acquired: 06:3 oct 13, 2005

Survey Co:US Geological Survey

Filename: $n t 55 n$.avg

Channels: Ch1 Ch2 Ch3 Ch4 Ch5 Ch3 Ch4

Plotted: 09:57 Feb 02, 2006

< EMI - ElectroMagnetic Instruments > 


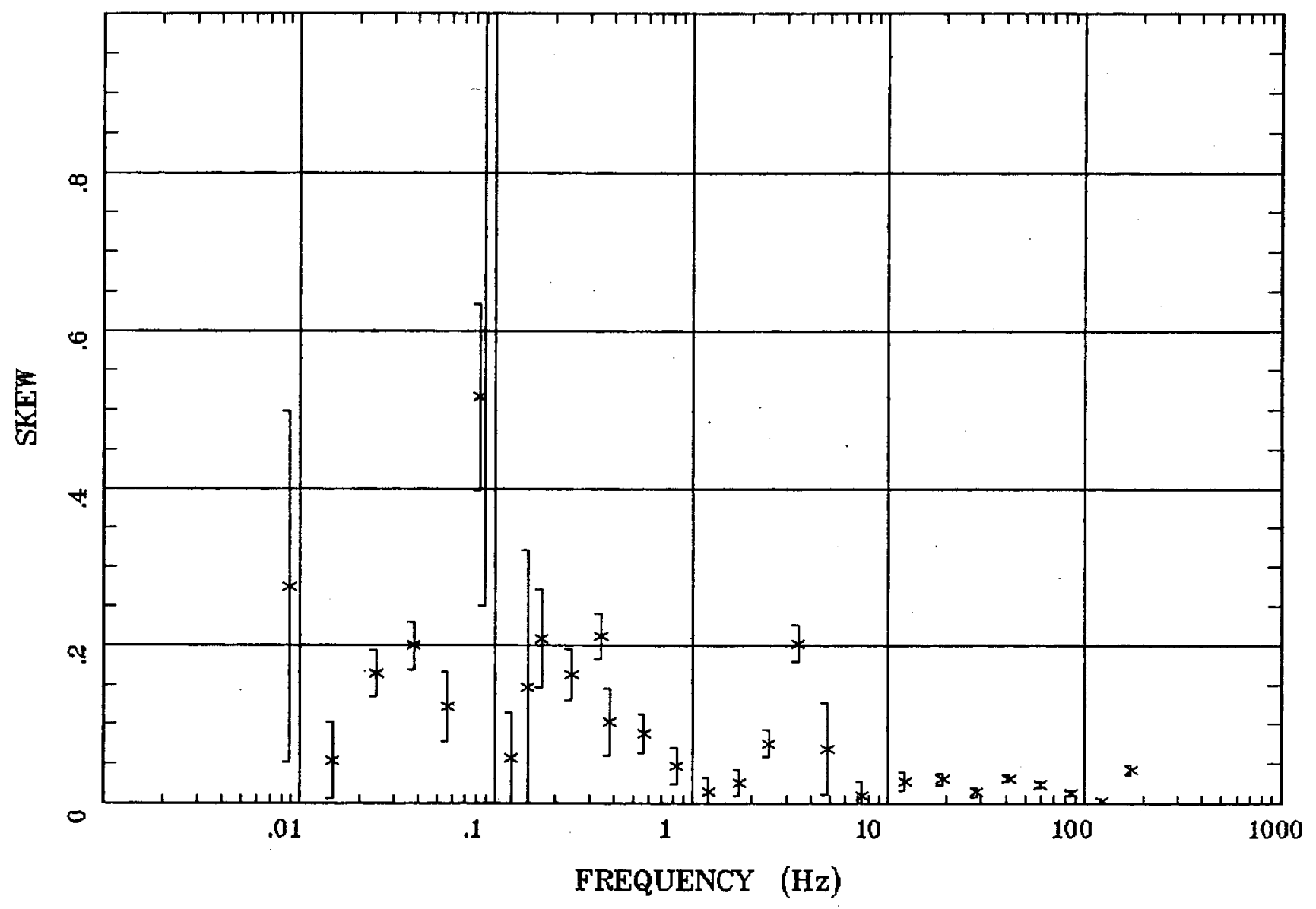

Client: TAter Resources \& DOD Remote: none

Acquired: 06:3 Oct 13, 2005

Survey Co:US Geological Survey
Rotation:

Filename: nt55n.avg

Channels: Ch1 Ch2 Ch3 Ch4 Ch5 Ch3 Ch4

Plotted: 09:57 Feb 02, 2006

< EMI - ElectroMagnetic Instruments > 


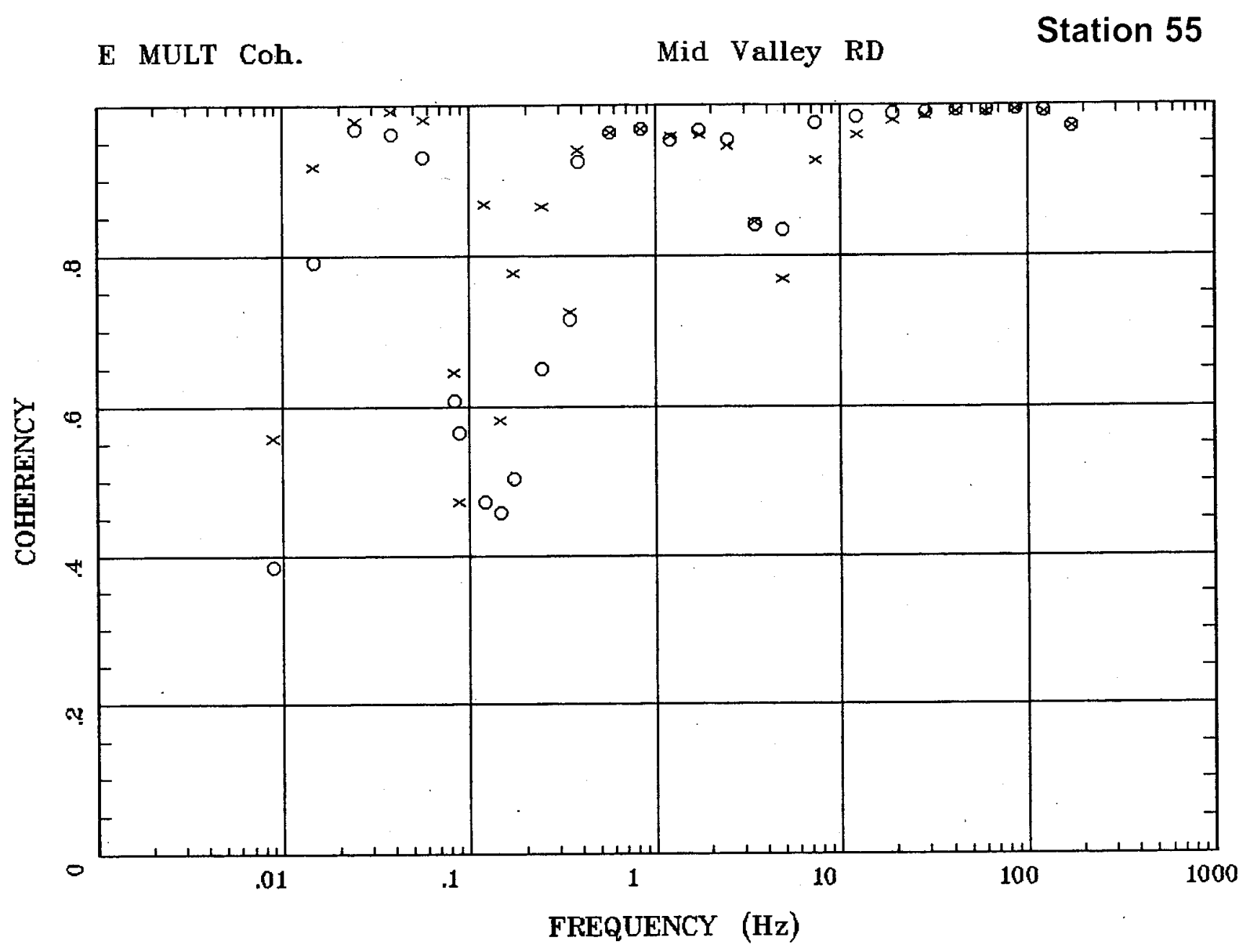

Client: FAter Resources \& DOD Remote: none

Acquired: $06: 3$ oct 13,2005 Survey Co:US Geological Survey
Rotation:

Filename: nt55n.avg Channels: Ch1 Ch2 Ch3 Ch4 Ch5 Ch3 Ch4 Plotted: 09:57 Feb 02, 2006

< EMI - ElectroMagnetic Instruments > 


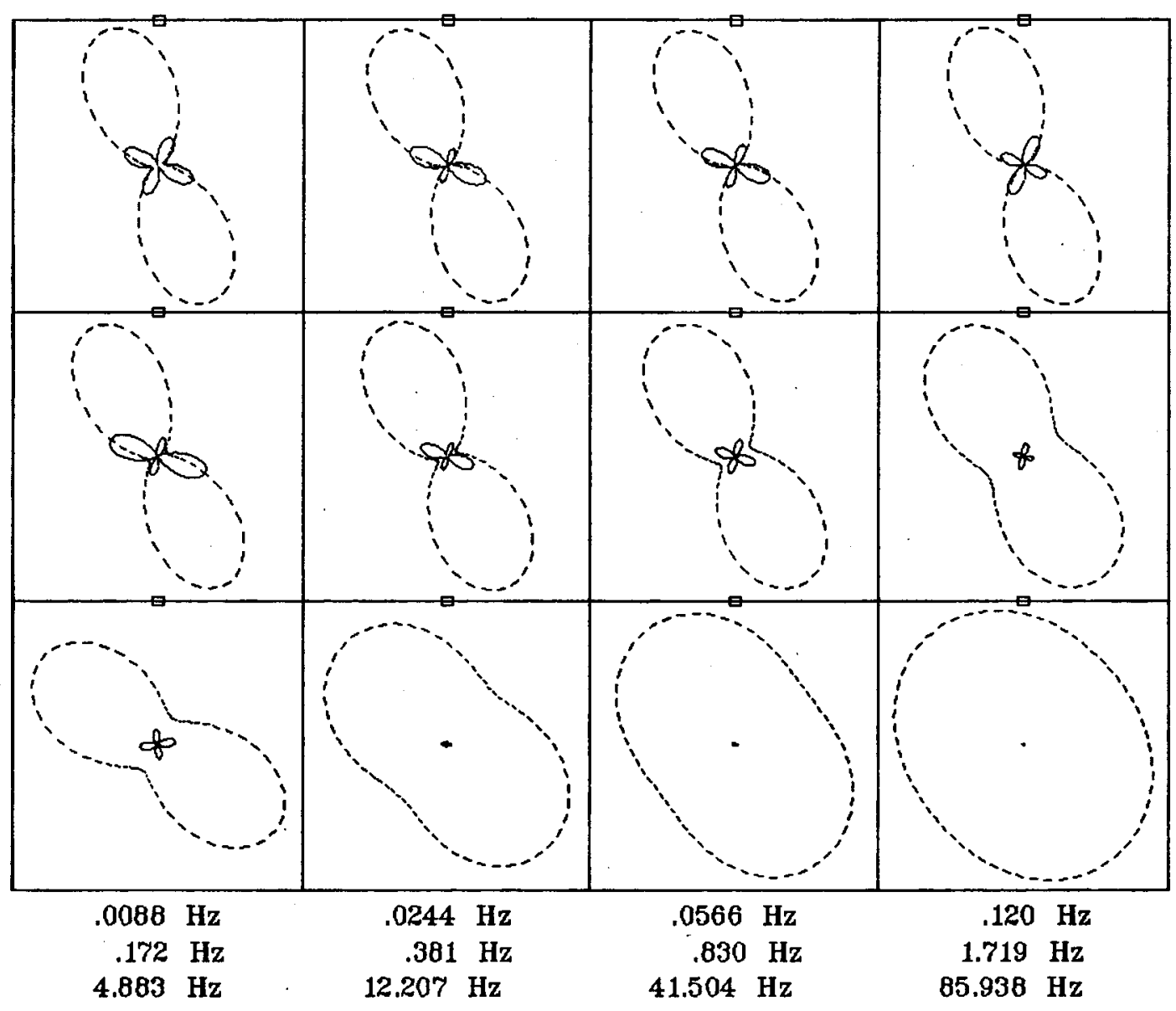

Client: WAter Resources \& DOD Remote: none Acquired: 06:3 oct 13, 2005 Survey Co:US Geological Survey
Rotation:

Filename: nt55n.avg Channels: Ch1 Ch2 Ch3 Ch4 Ch5 Ch3 Ch4 Plotted: 09:57 Feb 02, 2006

< EMI - ElectroMagnetic Instruments > 
TIPPER MAGNITUDE

Mid Valley RD

Station 55

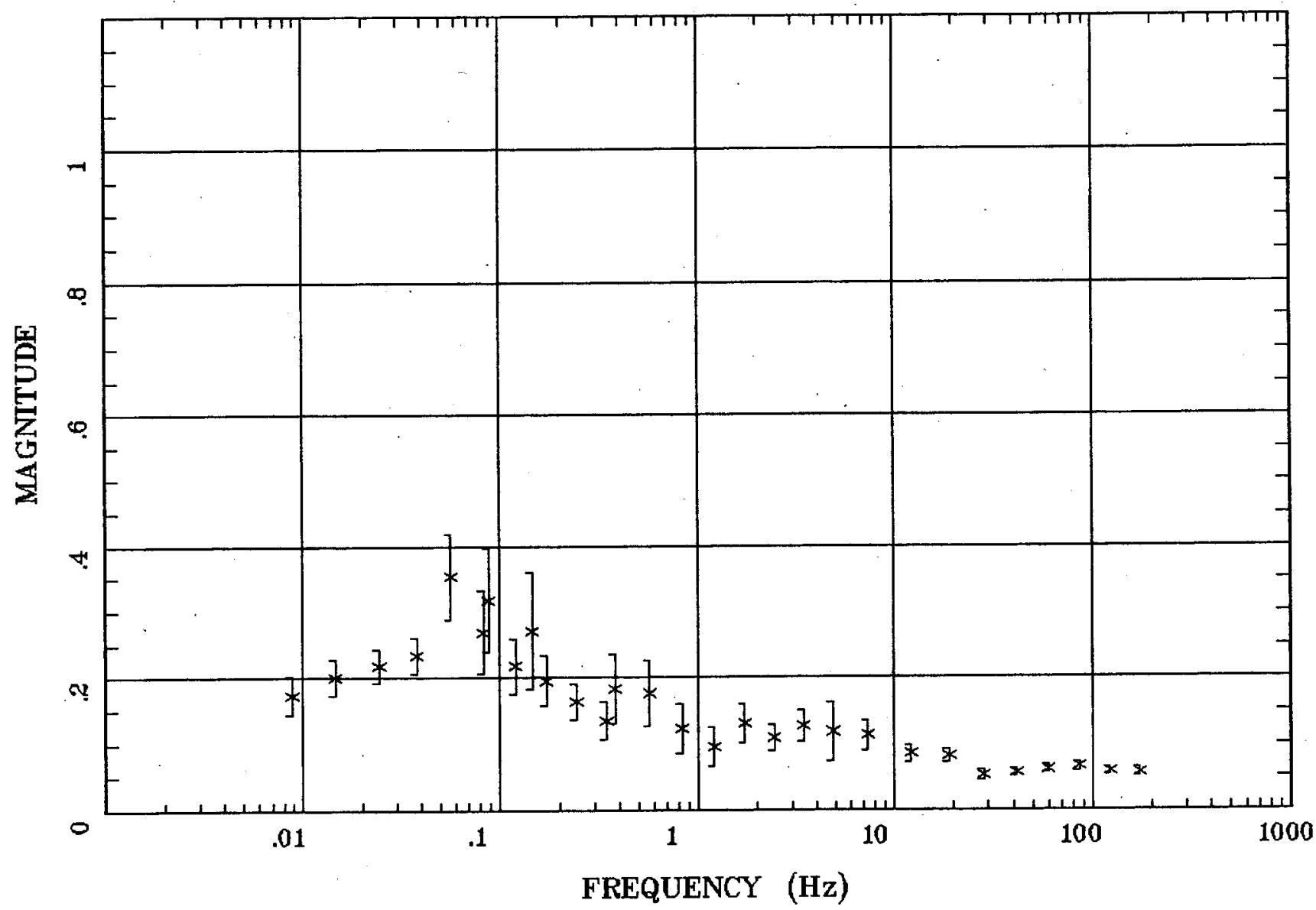

Client: WAter Resources \& DOD

Remote: none

Acquired: 06:3 oct 13, 2005

Survey Co:US Geological Survey

Rotation:

Filename: nt55n.avg

Channels: Ch1 Ch2 Ch3 Ch4 Ch5 Ch3 Ch4

Plotted: 09:57 Feb 02, 2006

< EMI - ElectroMagnetic Instruments > 
TIPPER STRIKE

Mid Valley RD

Station 55

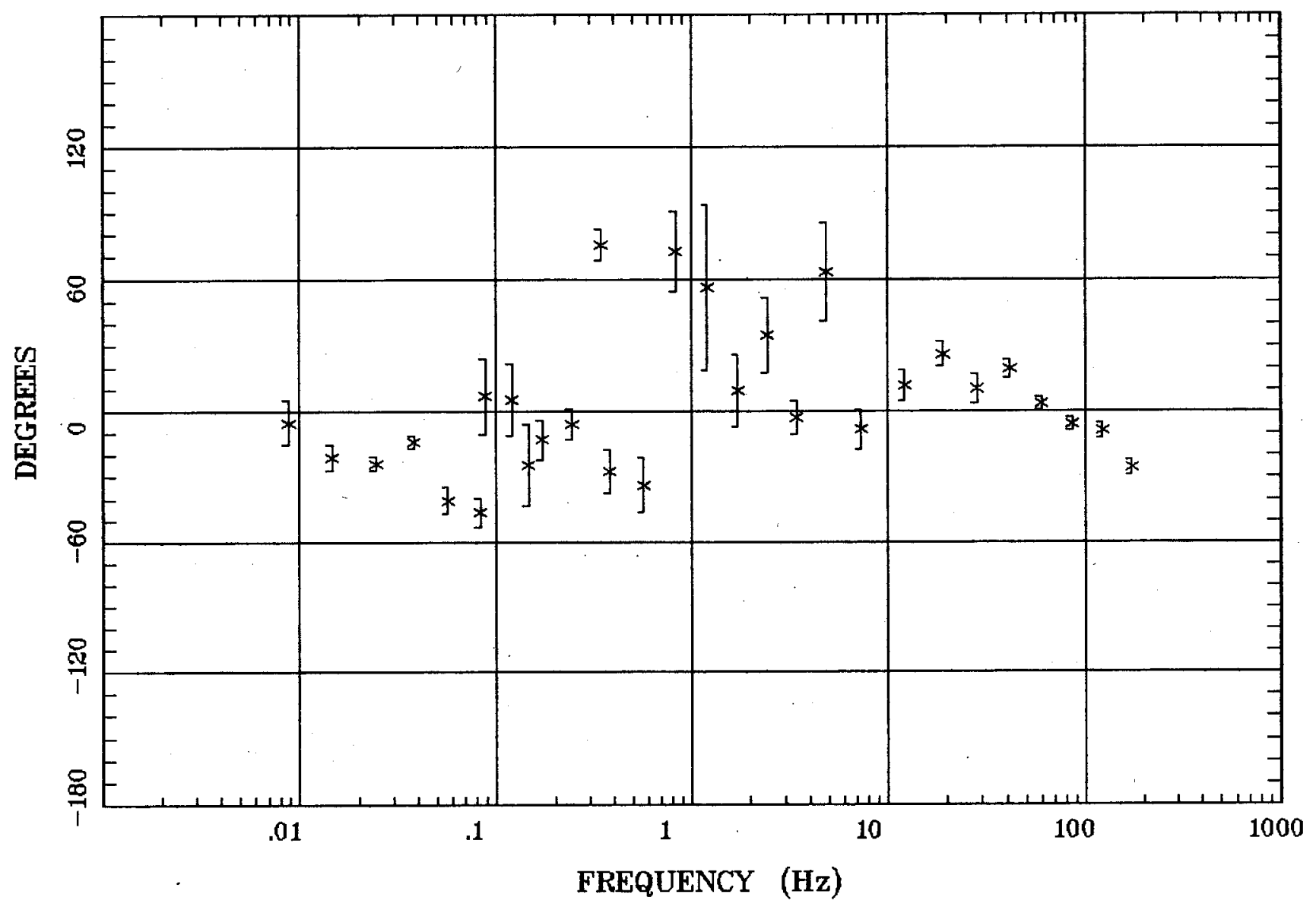

Client: WAter Resources \& DOD

Rotation:

Remote: none

Acquired: $06: 3$ Oct 13, 2005

Filename: nt55n.avg

Channels: Ch1 Ch2 Ch3 Ch4 Ch5 Ch3 Ch4

Plotted: 09:57 Feb 02, 2006

Survey Co:US Geological Survey

< EMI - ElectroMagnetic Instruments > 
HzHx.x Coh HzHy.o

Mid Valley RD

Station 55

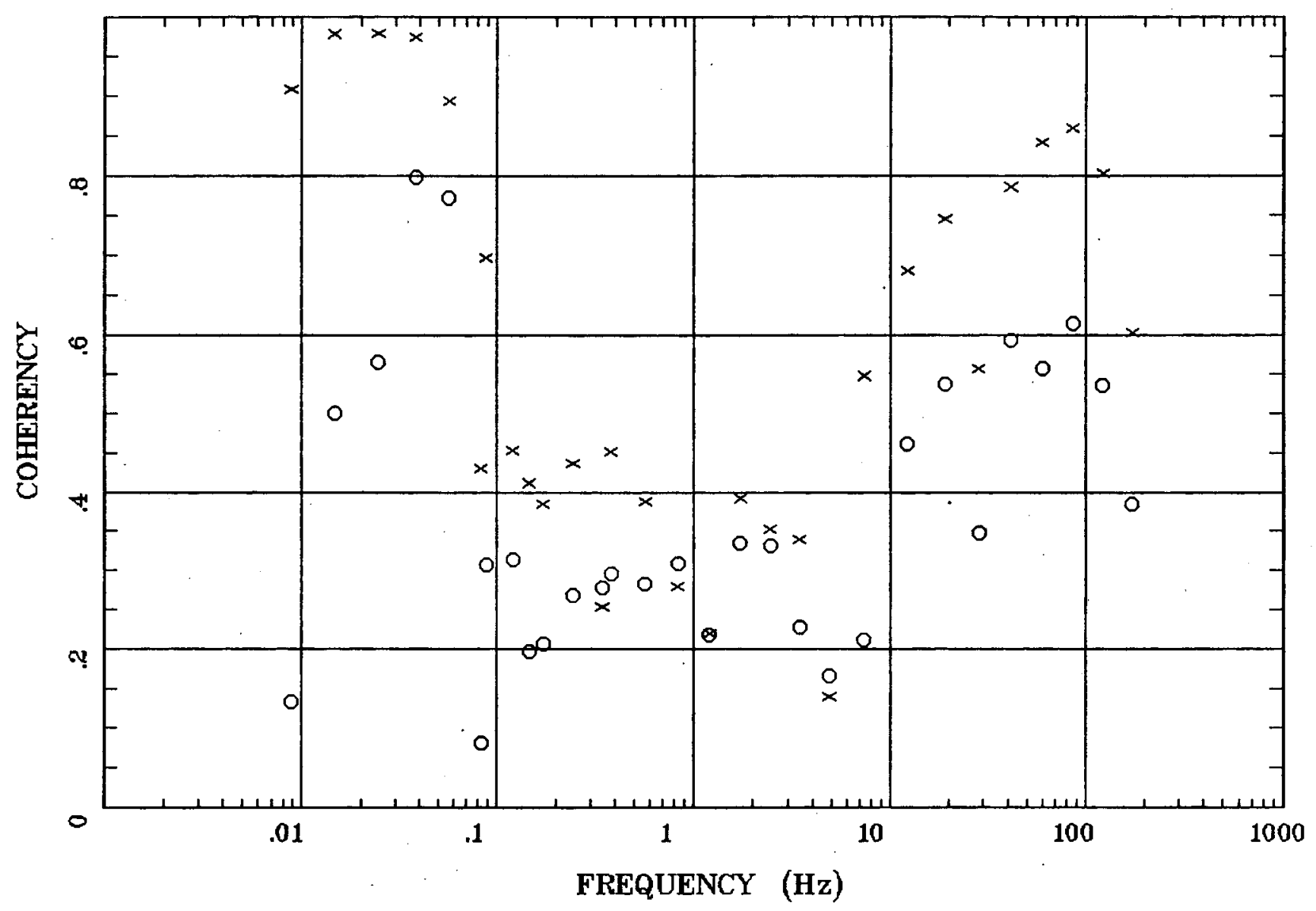

Client: WAter Resources \& DOD

Rotation:

Remote: none

Acquired: 06:3 oct 13, 2005

Survey Co:US Geological Survey
Filename: nt55n.avg

Channels: Ch1 Ch2 Ch3 Ch4 Ch5 Ch3 Ch4 Plotted: 09:57 Feb 02, 2006

< EMI - ElectroMagnetic Instruments > 


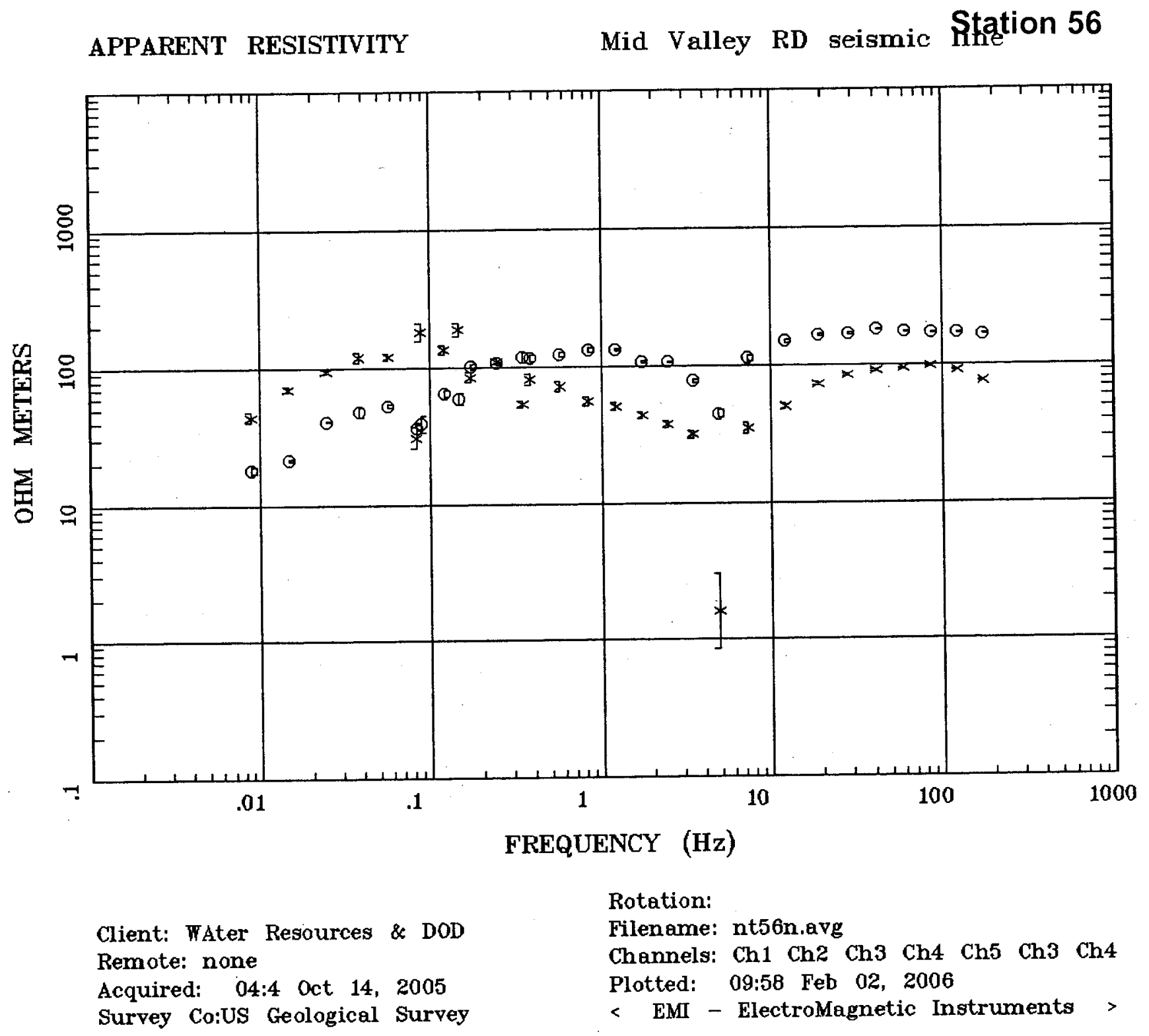


IMPEDANCE PHASE

Mid Valley $R D$ seismic line

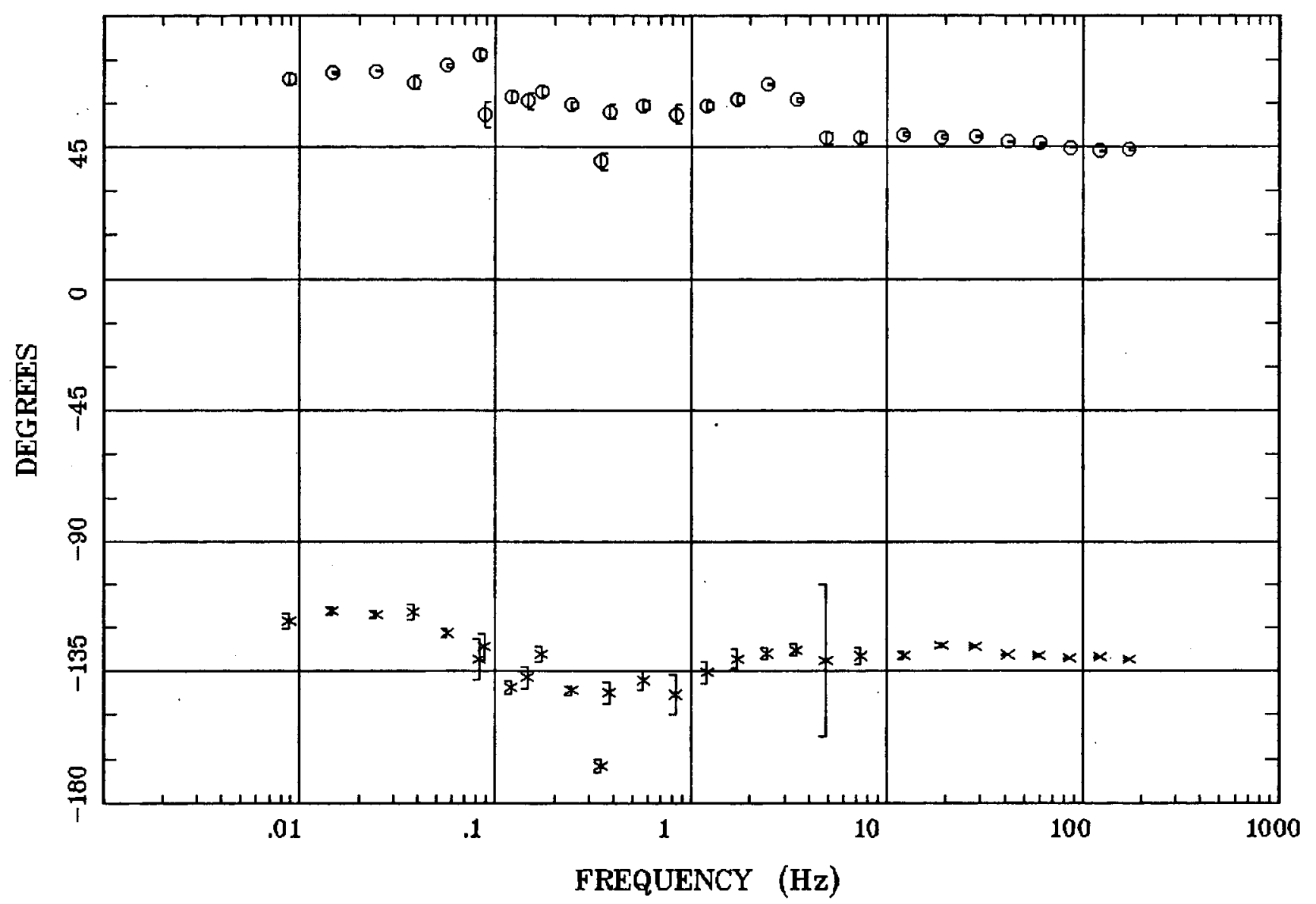

Client: WAter Resources \& DOD Remote: none

Acquired: 04:4 Oct 14, 2005 Survey Co:US Geological Survey
Rotation:

Filename: nt56n.avg Channels: Ch1 Ch2 Ch3 Ch4 Ch5 Ch3 Ch4 Plotted: 09:58 Feb 02, 2006

< EMI - ElectroMagnetic Instruments > 
ROTATION ANGLE

Mid Valley RD seismic line

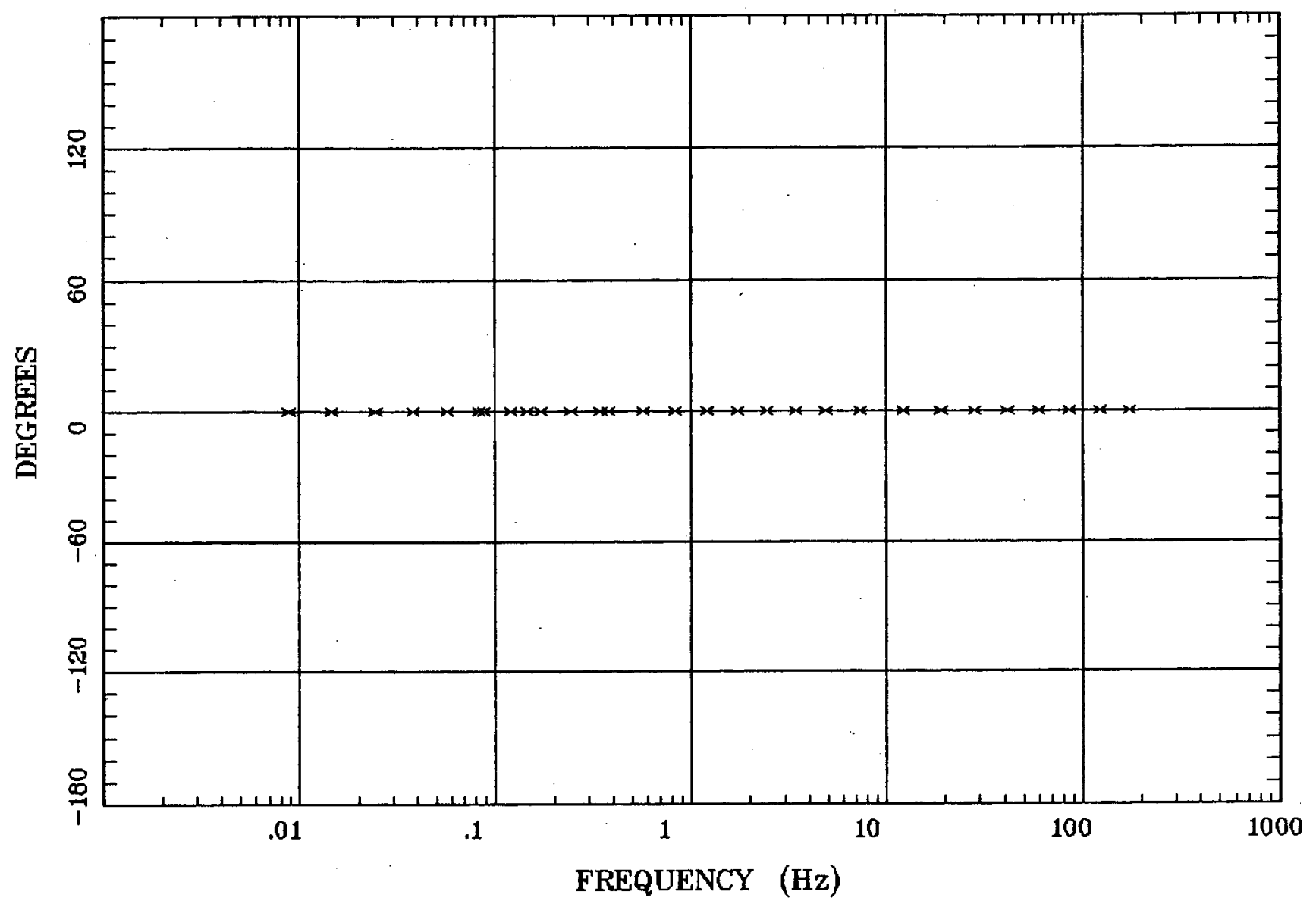

Client: WAter Resources \& DOD Remote: none Acquired: 04:4 oct 14, 2005 Survey Ca:US Geological Survey
Rotation:

Filename: nt56n.avg Channels: Ch1 Ch2 Ch3 Ch4 Ch5 Ch3 Ch4 Plotted: 09:58 Feb 02, 2006

< EMI - ElectroMagnetic Instruments > 


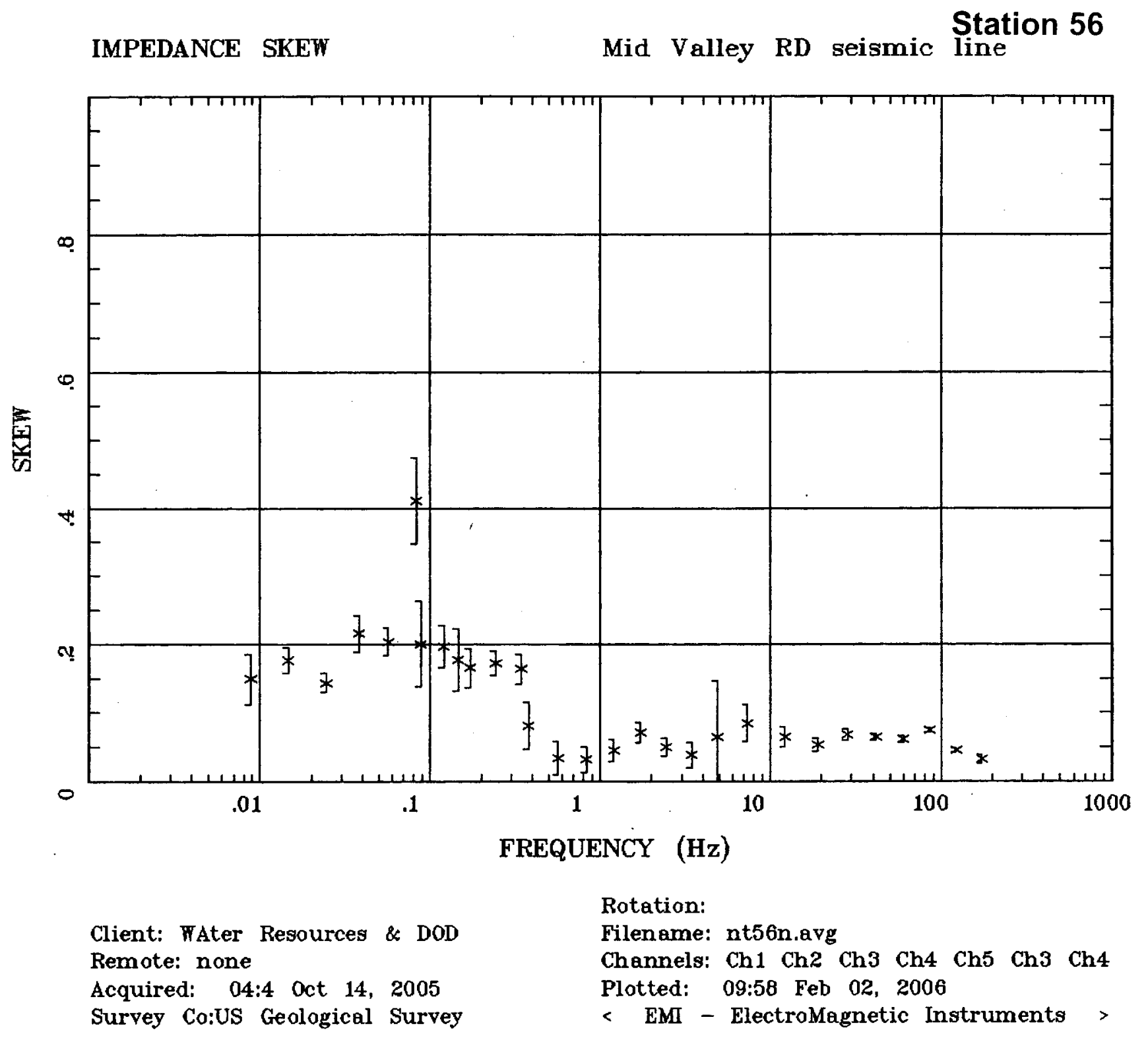


E MULT Coh.

Station 56

Mid Valley $R D$ seismic line

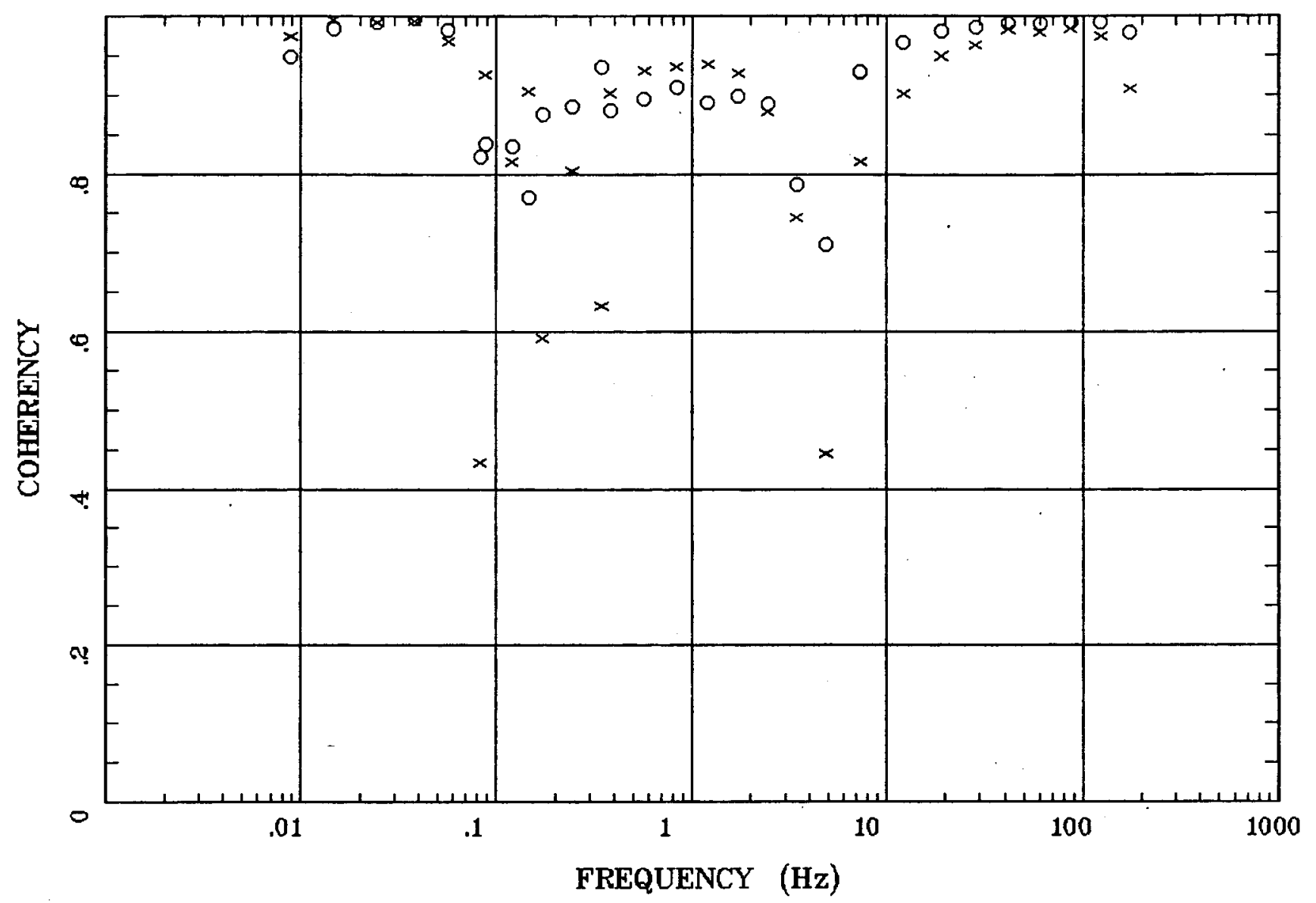

Client: WAter Resources \& DOD

Rotation:

Filename: nt56n.avg

Remote: none

Acquired: $04: 4$ oct 14, 2005

Channels: Ch1 Ch2 Ch3 Ch4 Ch5 Ch3 Ch4

Plotted: 09:58 Feb 02, 2006

Survey Co:US Geological Survey

< EMI - ElectroMagnetic Instruments > 


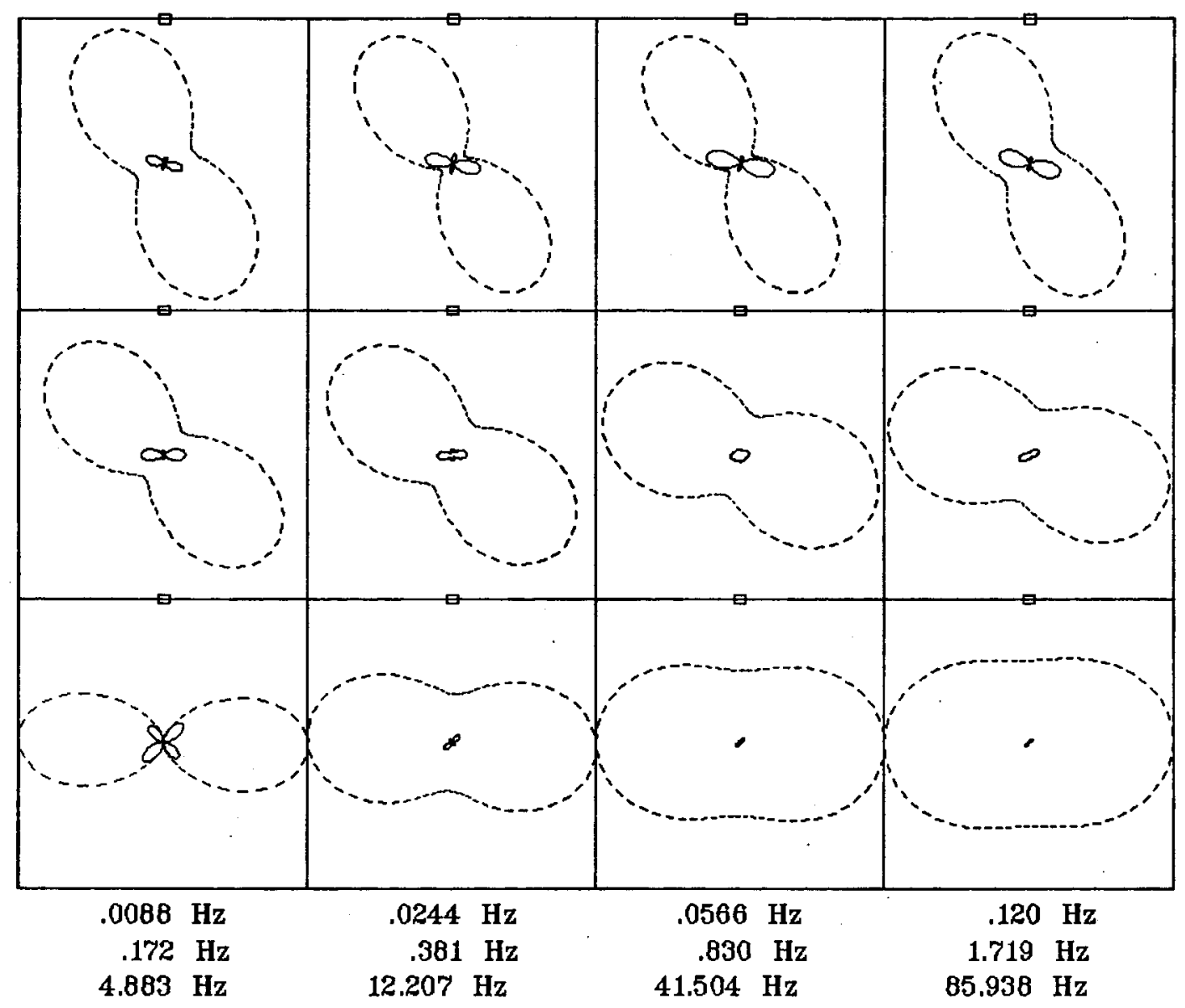

Rotation:

Client: WAter Resources \& DOD Remote: none

Filename: nt56n.avg

Channels: $\mathrm{Ch} 1 \mathrm{Ch} 2 \mathrm{Ch} 3 \mathrm{Ch} 4 \mathrm{Ch} 5 \mathrm{Ch} 3 \mathrm{Ch} 4$

Acquired: $04: 4$ oct 14, 2005

Plotted: 09:58 Feb 02, 2006

Survey Co:US Geological Survey

< EMI - ElectroMagnetic Instruments > 
TIPPER MAGNITUDE

Mid Valley RD seismic Station 56

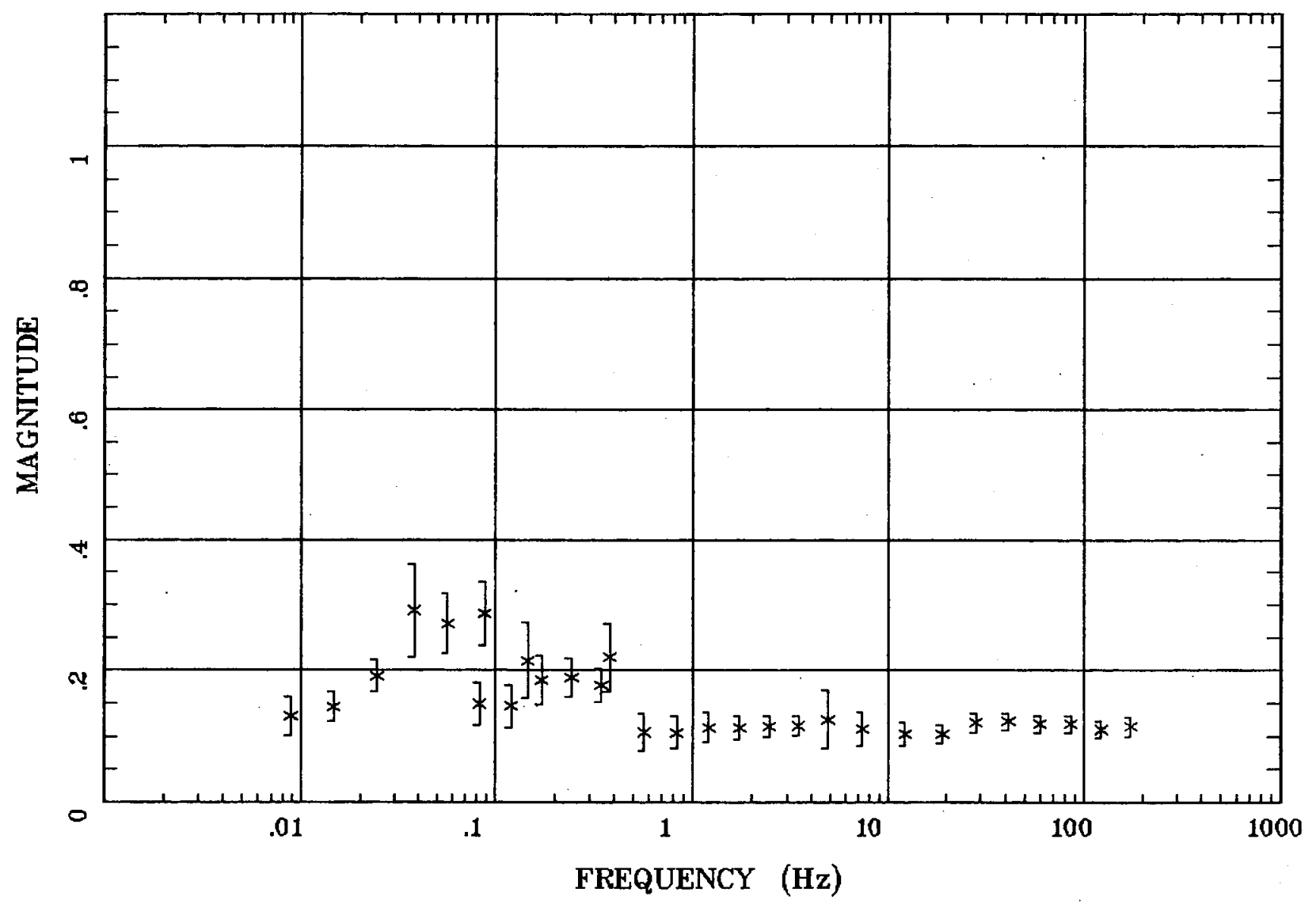

Client: WAter Resources \& DOD Remote: none

Acquired: 04:4 Oct 14, 2005 Survey Co:US Geological Survey
Rotation:

Filename: nt56n.avg

Channels: Ch1 Ch2 Ch3 Ch4 Ch5 Ch3 Ch4

Plotted: 09:58 Feb 02, 2006

< EMI - ElectroMagnetic Instruments > 


\section{TIPPER STRIKE}

Mid Valley $R D$ seismic line

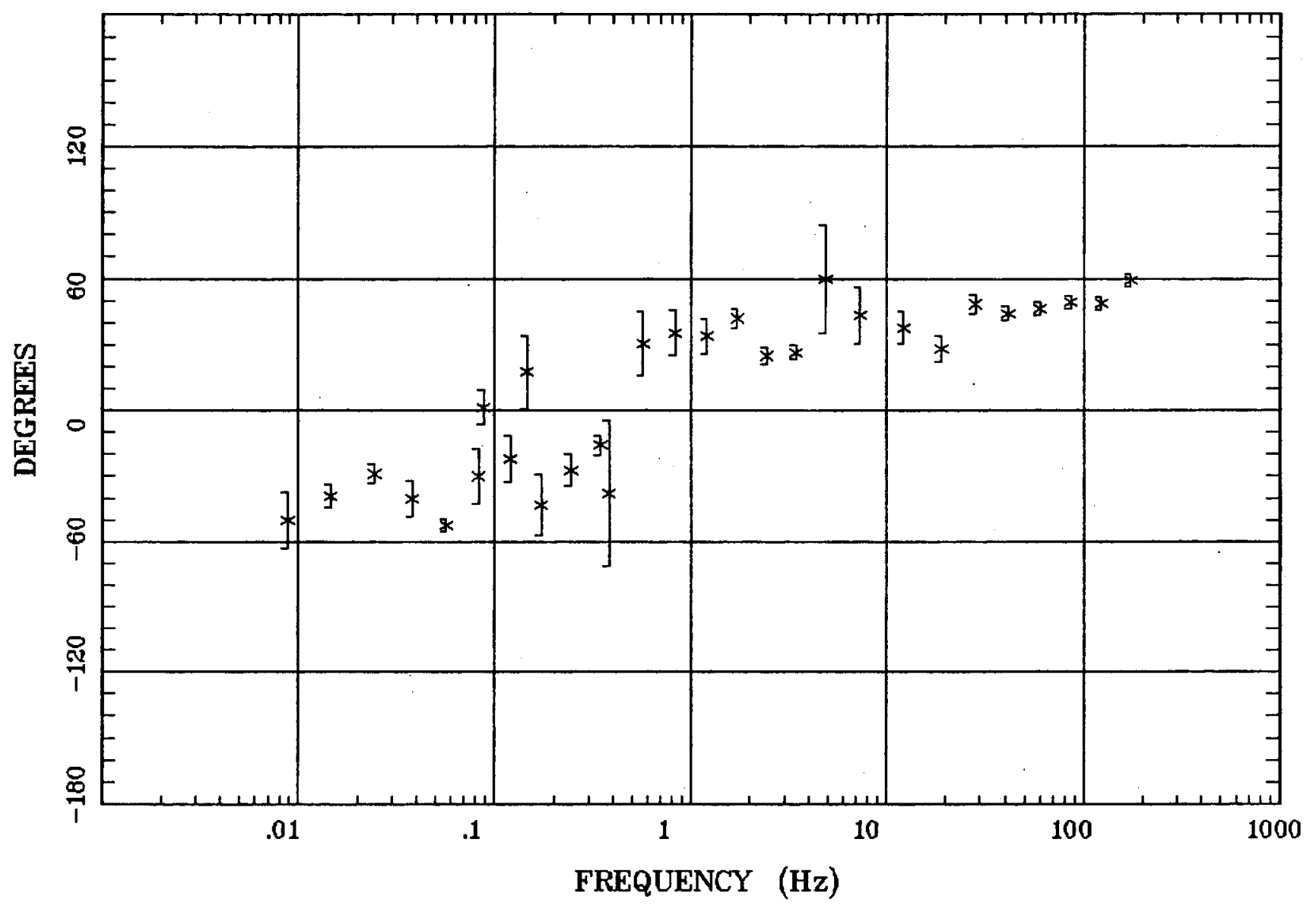

Client: WAter Resources \& DOD Remote: none

Acquired: 04:4 Oct 14, 2005

Survey Co:US Geological Survey

Rotation:

Filename: nt56n.avg

Channels: Ch1 Ch2 Ch3 Ch4 Ch5 Ch3 Ch4

Plotted: 09:58 Feb 02, 2006

< EMI - ElectroMagnetic Instruments > 


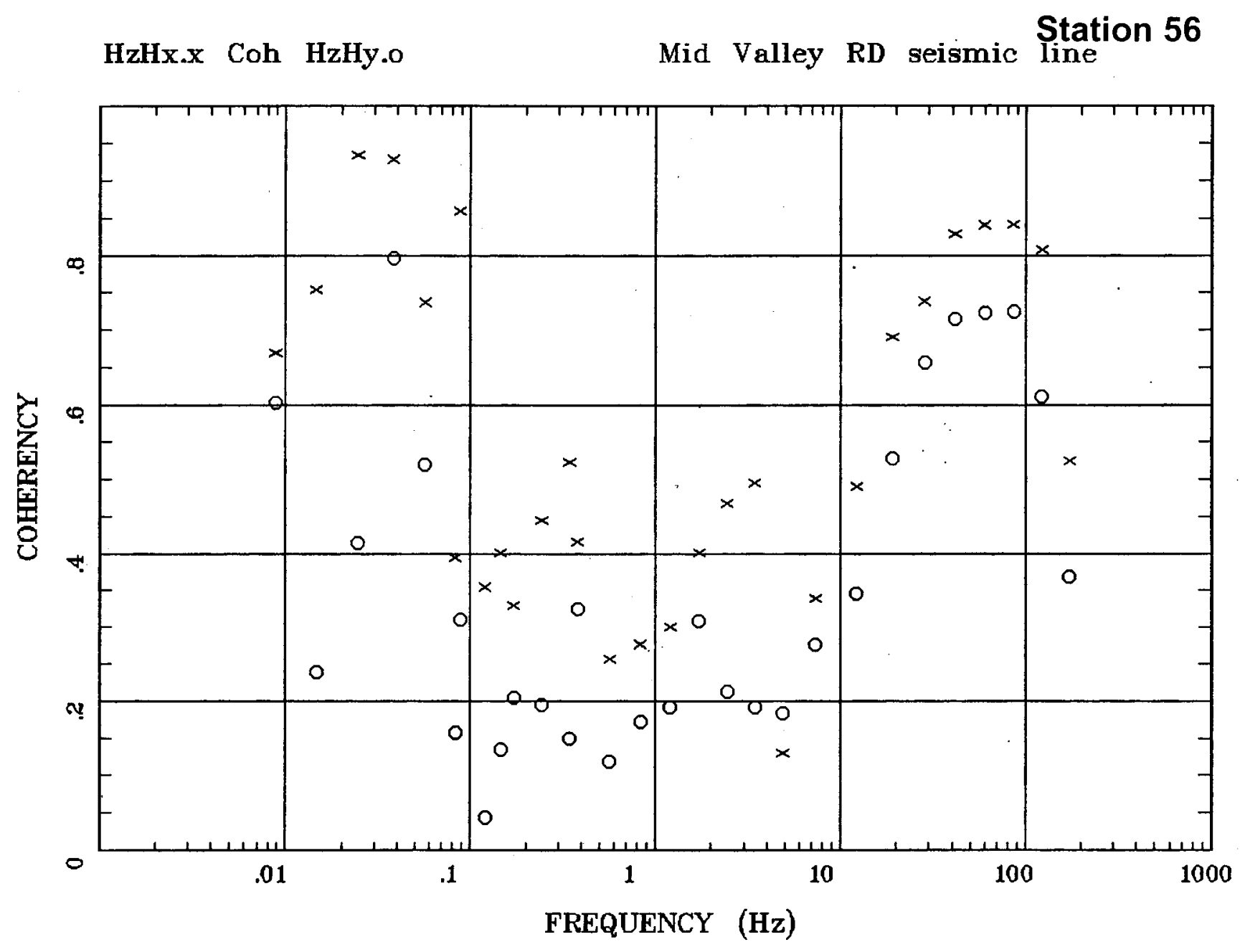

Client: WAter Resources \& DOD Remote: none

Acquired: 04:4 oct 14, 2005 Survey Co:US Geological Survey

Rotation:

Filename: nt56n.avg

Channels: Ch1 Ch2 Ch3 Ch4 Ch5 Ch3 Ch4

Plotted: 09:58 Feb 02, 2006

< EMI - ElectroMagnetic Instruments > 


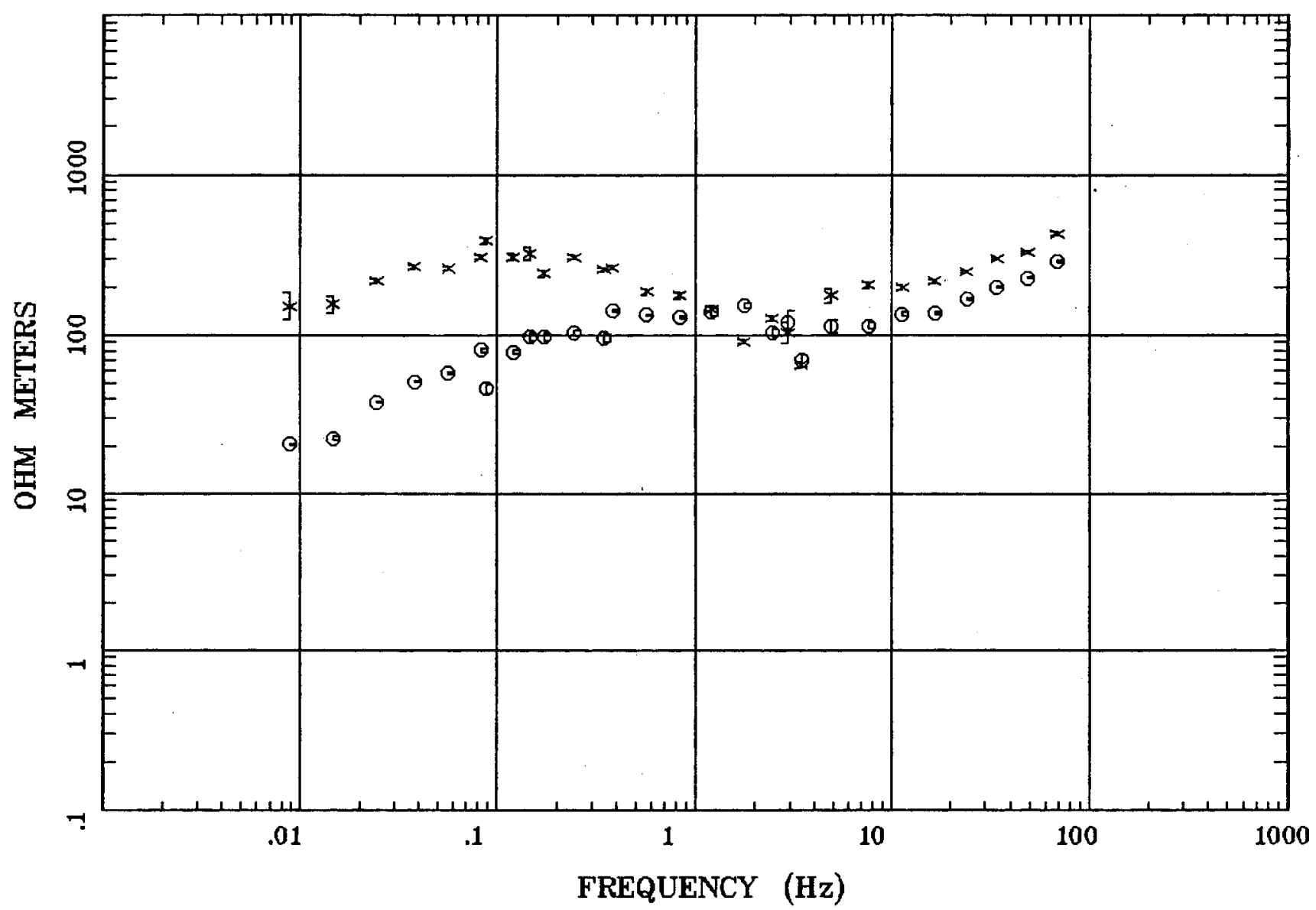

Client: WAter Resources \& DOD Remote: none Acquired: 08:5 oct 16, 2005 Survey Co:US Geological Survey
Rotation:

Filename: nt57n.avg

Channels: Ch1 Ch2 Ch3 Ch4 Ch5 Ch3 Ch4 Plotted: 10:42 Feb 02, 2006

< EMI - ElectroMagnetic Instruments > 


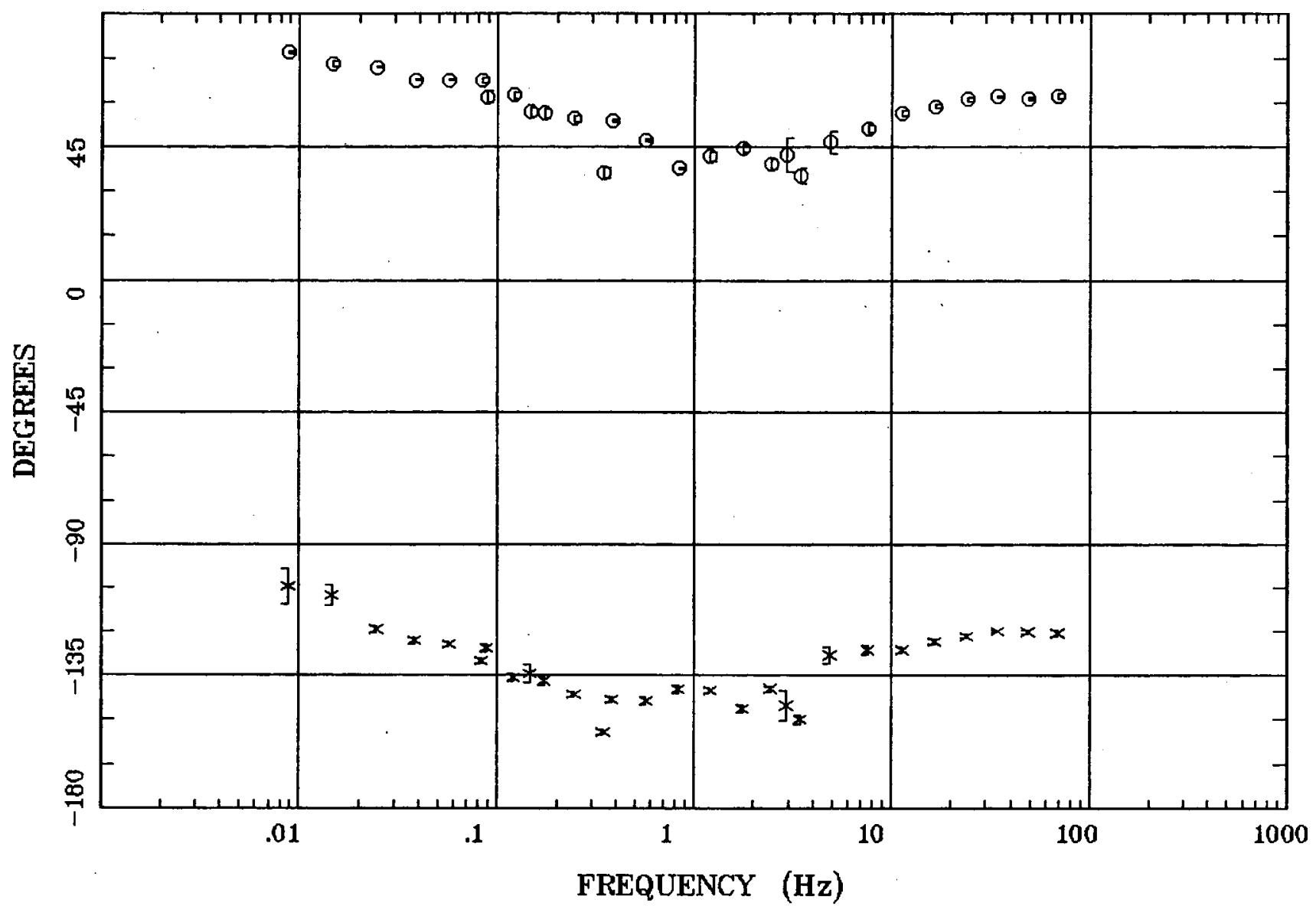

Client: WAter Resources \& DOD Remote: none

Acquired: 08:5 oct 16, 2005 Survey Ca:US Geological Survey
Rotation:

Filename: nt57n.avg Channels: Ch1 Ch2 Ch3 Ch4 Ch5 Ch3 Ch4 Plotted: 10:42 Feb 02, 2006

< EMI - ElectroMagnetic Instruments > 


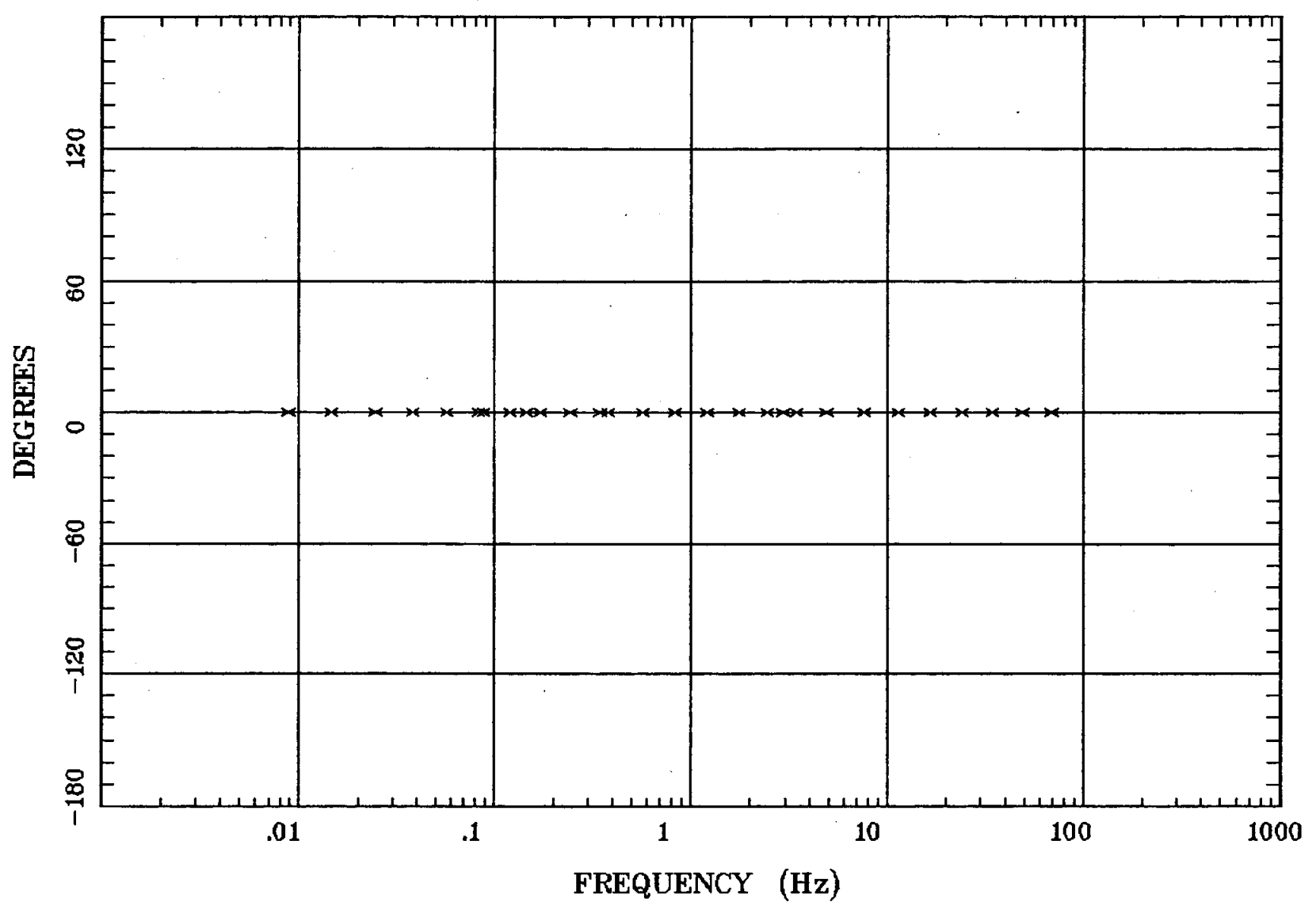

Client: WAter Resources \& DOD Remote: none Acquired: 08:5 oct 16, 2005 Survey Co:US Geological Survey
Rotation:

Filename: nt57n.avg Channels: Ch1 Ch2 Ch3 Ch4 Ch5 Ch3 Ch4 Plotted: 10:42 Feb 02, 2006

< EMI - ElectroMagnetic Instruments > 


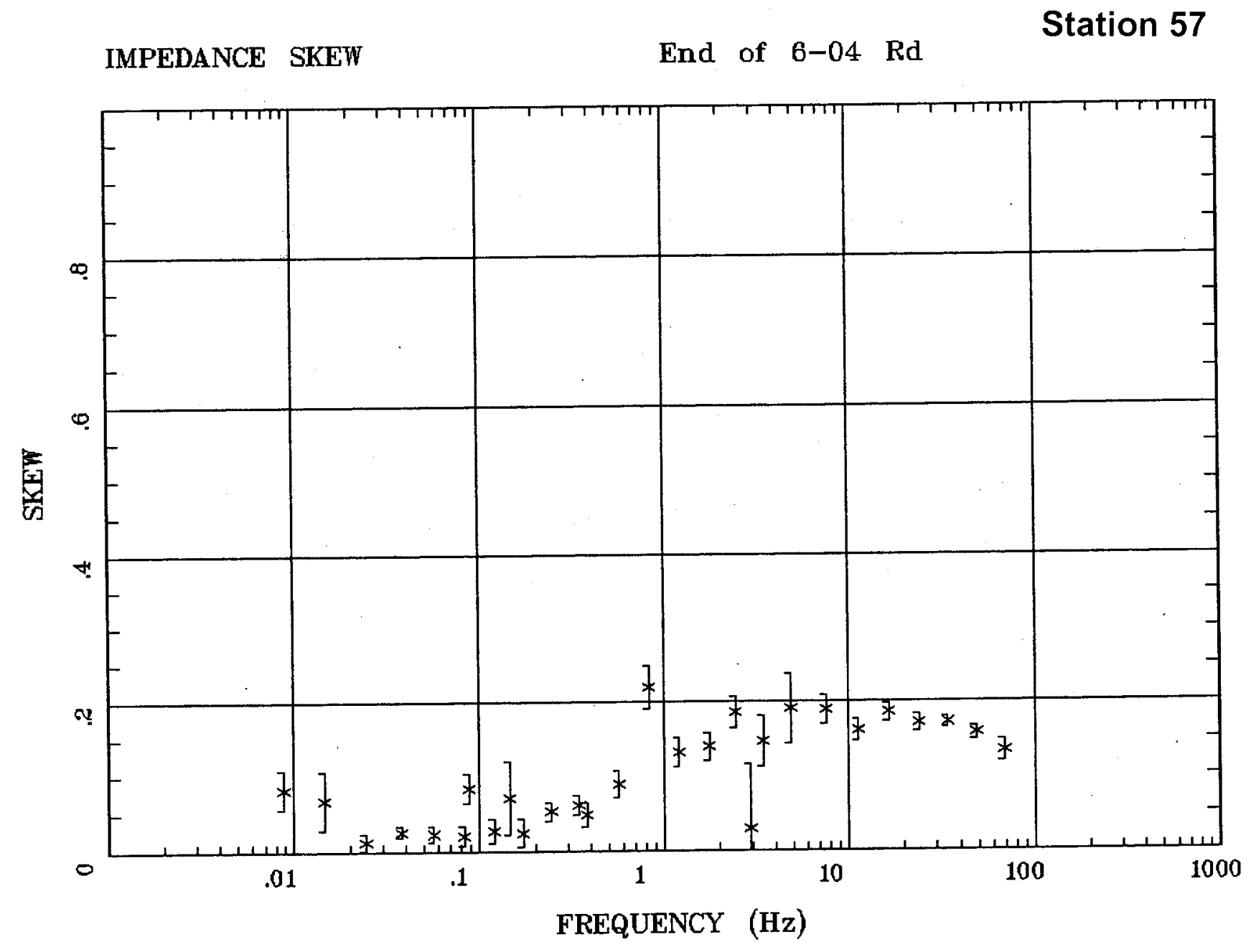

Client: WAter Resources \& DOD Remote: none

Acquired: 08:5 oct 16, 2005 Survey Co:US Geological Survey
Rotation:

Filename: nt57n.evg Channels: Ch1 Ch2 Ch3 Ch4 Ch5 Ch3 Ch4 Plotted: 10:42 Feb 02, 2006

< EMI - ElectroMagnetic Instruments > 


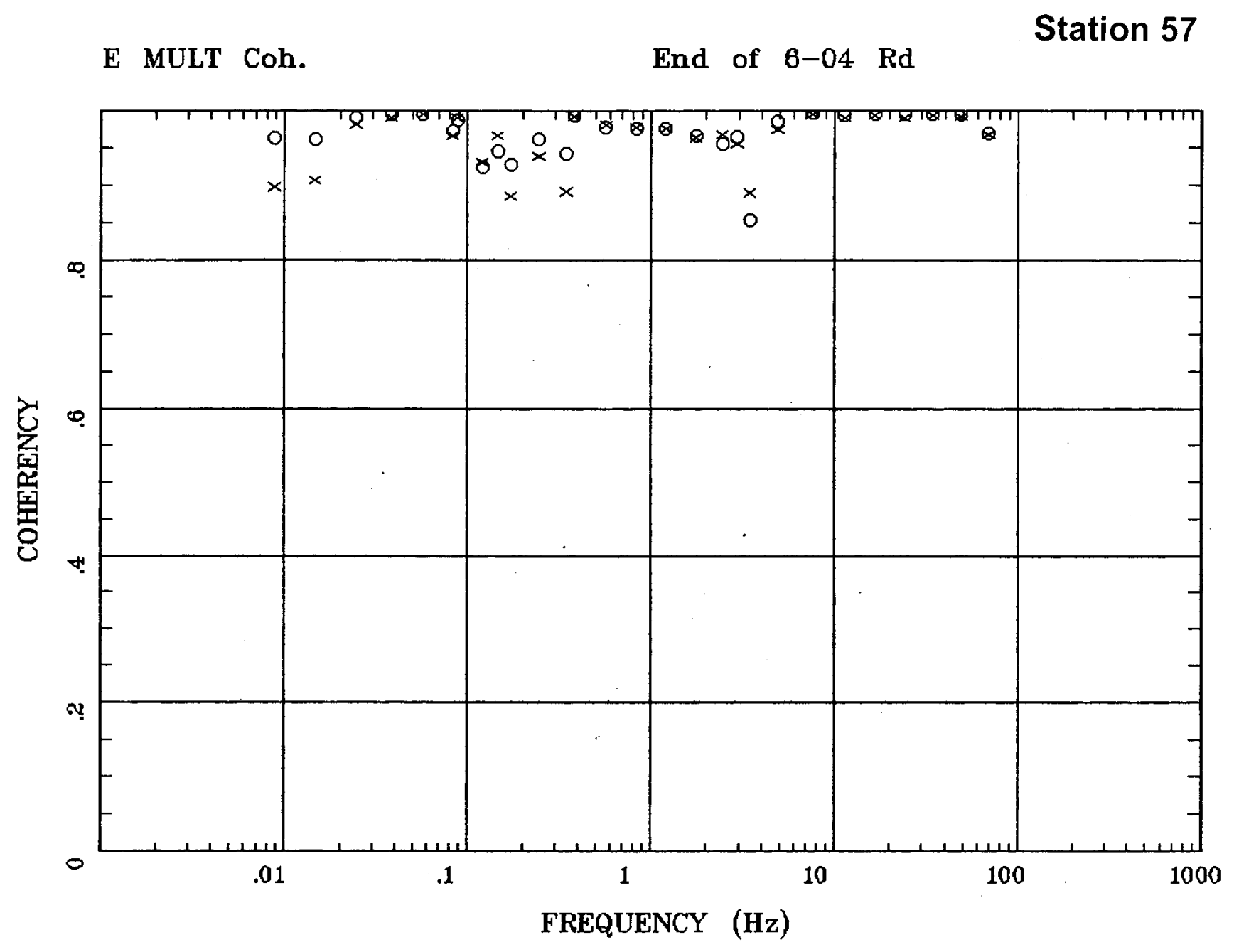

Client: FAter Resources \& DOD Remote: none

Acquired: 08:5 oct 16, 2005 Survey Co:US Geological Survey
Rotation:

Filename: nt57n.evg Channels: Ch1 Ch2 Ch3 Ch4 Ch5 Ch3 Ch4 Plotted: 10:42 Feb 02, 2006

< EMI - ElectroMagnetic Instruments > 


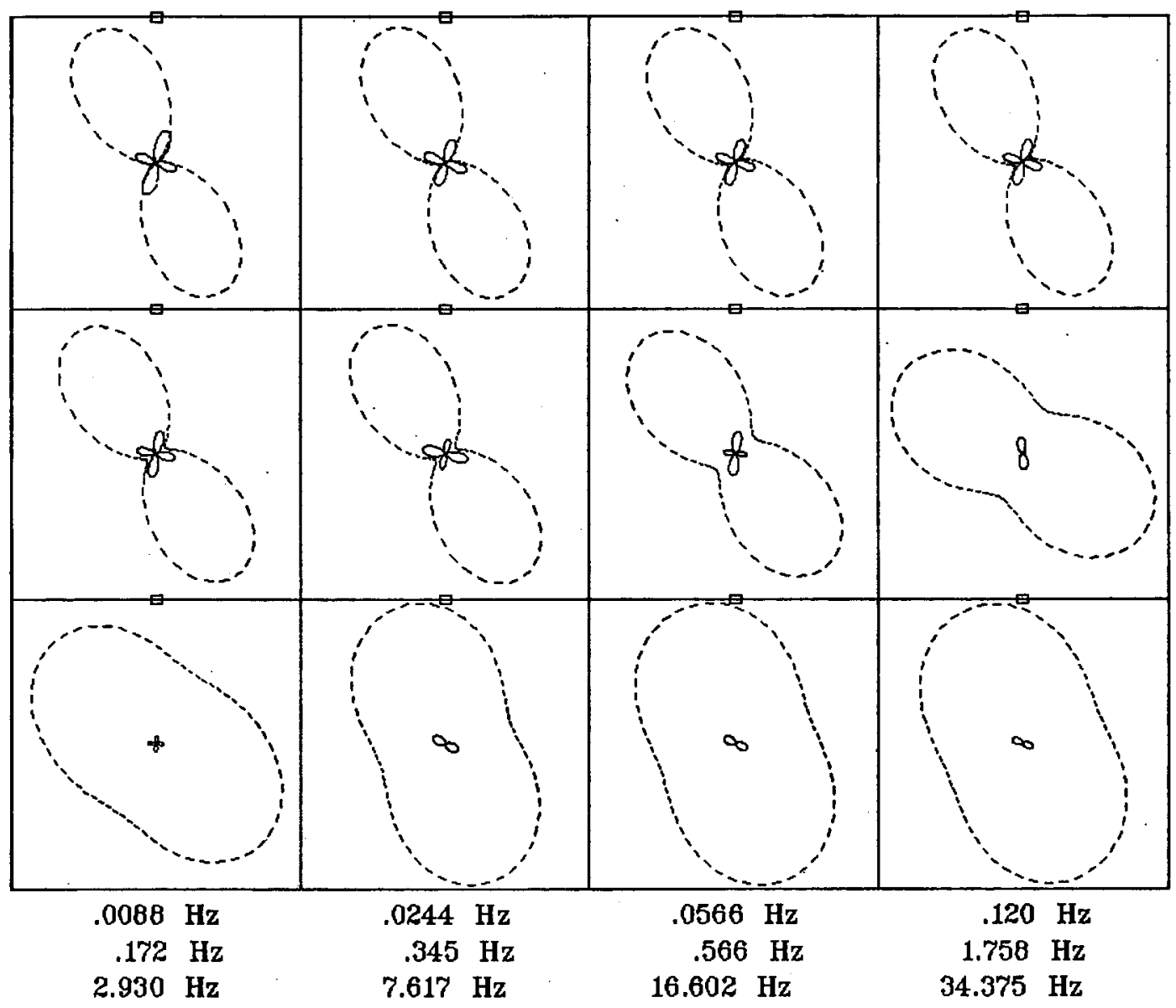

Client: FAter Resources \& DOD Remote: none

Acquired: 08:5 Oct 16, 2005 Survey Co:US Geological Survey
Rotation:

Filename: nt57n.avg Channels: Ch1 Ch2 Ch3 Ch4 Ch5 Ch3 Ch4 Platted: 10:42 Feb 02, 2006

< EMI - ElectroMagnetic Instruments > 
TIPPER MAGNITUDE

End of 6-04 Rd

Station 57

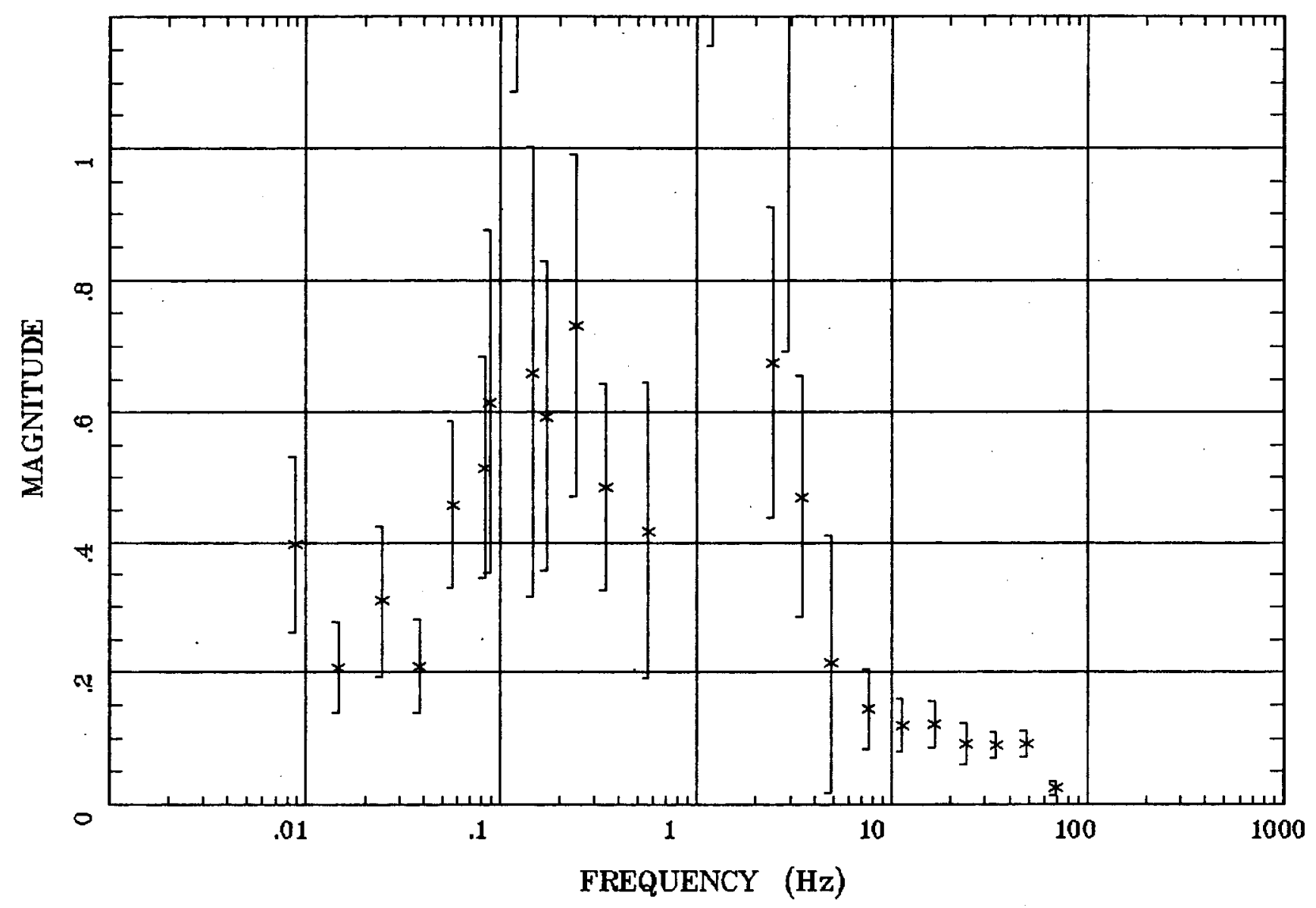

Client: WAter Resources \& DOD Remote: none Acquired: 08:5 Oct 16, 2005 Survey Co:US Geological Survey
Rotation:

Filename: nt57n.avg

Channels: Ch1 Ch2 Ch3 Ch4 Ch5 Ch3 Ch4

Plotted: 10:42 Feb 02, 2006

< EMI - ElectroMagnetic Instruments > 
TIPPER STRIKE

End of 6-04 Rd

Station 57

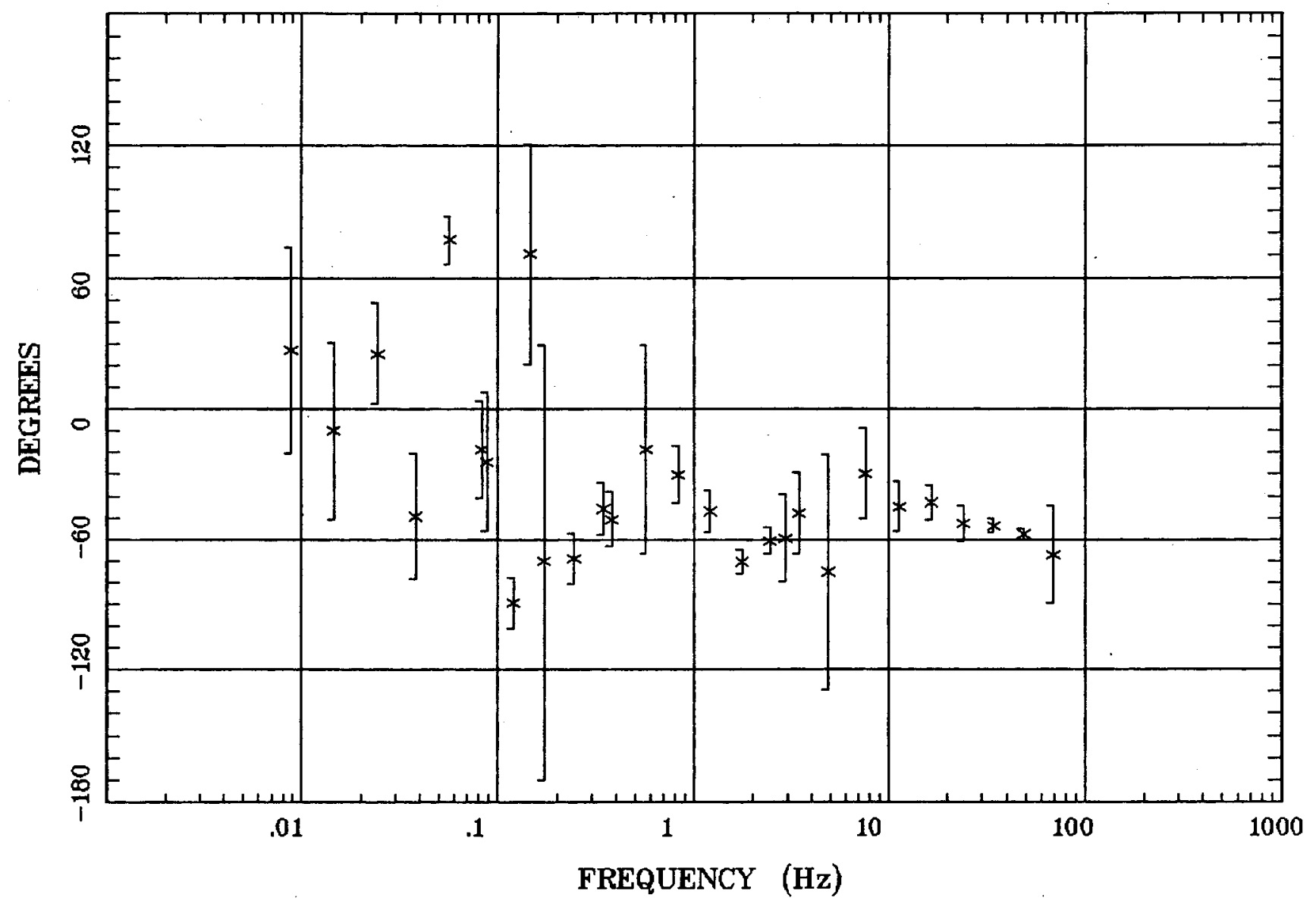

Client: WAter Resources \& DOD

Rotation:

Filename: nt57n.avg

Channels: Ch1 Ch2 Ch3 Ch4 Ch5 Ch3 Ch4 Plotted: 10:42 Feb 02, 2006

Acquired: 08:5 oct 16, 2005

Survey Co:US Geological Survey

< EMI - ElectroMagnetic Instruments > 

HzHx.x Coh HzHy.o
Station 57

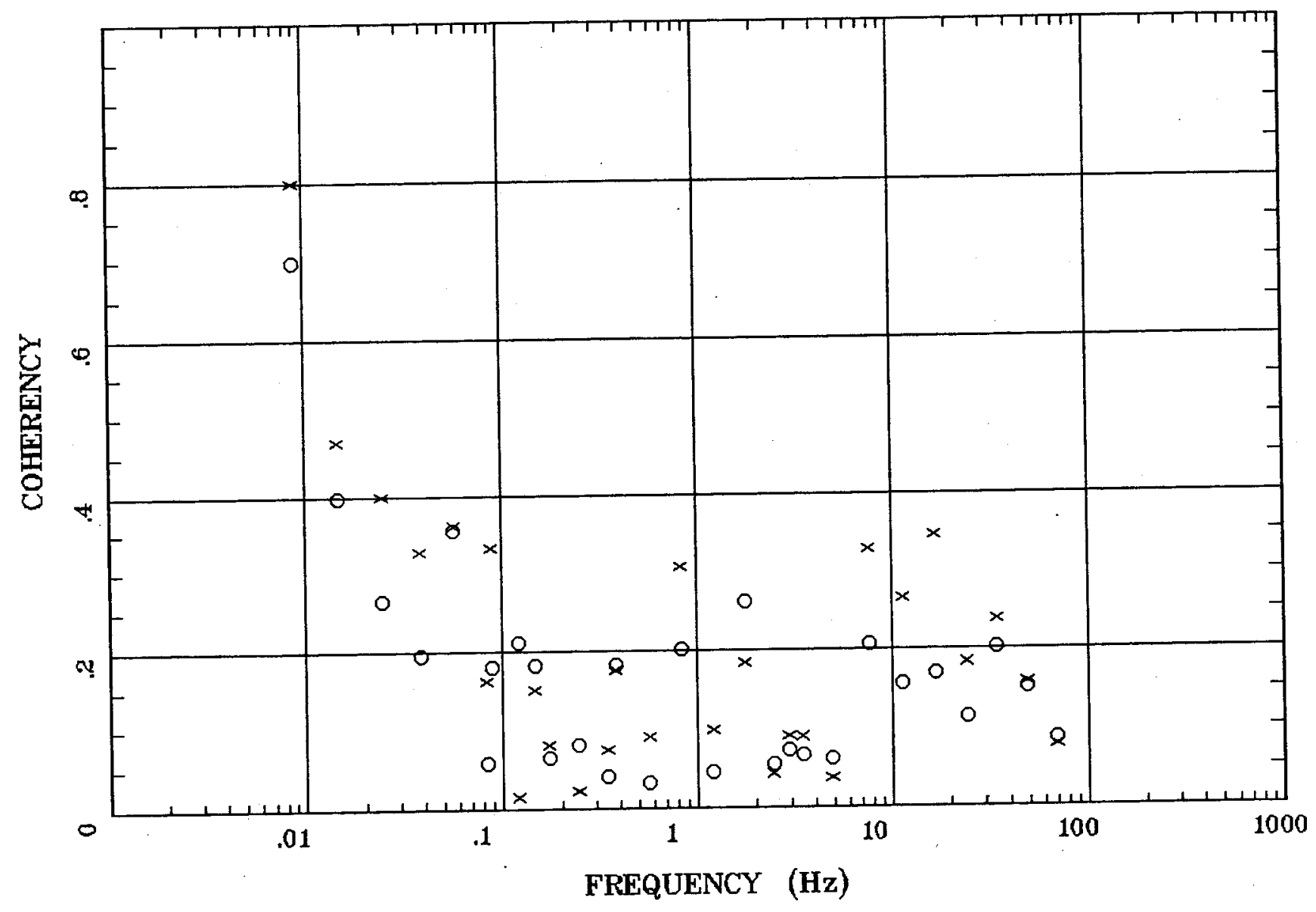

Client: WAter Resources \& DOD Remote: none Acquired: 08:5 oct 16, 2005 Survey Co:US Geological Survey
Rotation:

Filename: nt57n.avg Channels: Ch1 Ch2 Ch3 Ch4 Ch5 Ch3 Ch4 Plotted: 10:42 Feb 02, 2006

< EMI - ElectroMagnetic Instruments > 


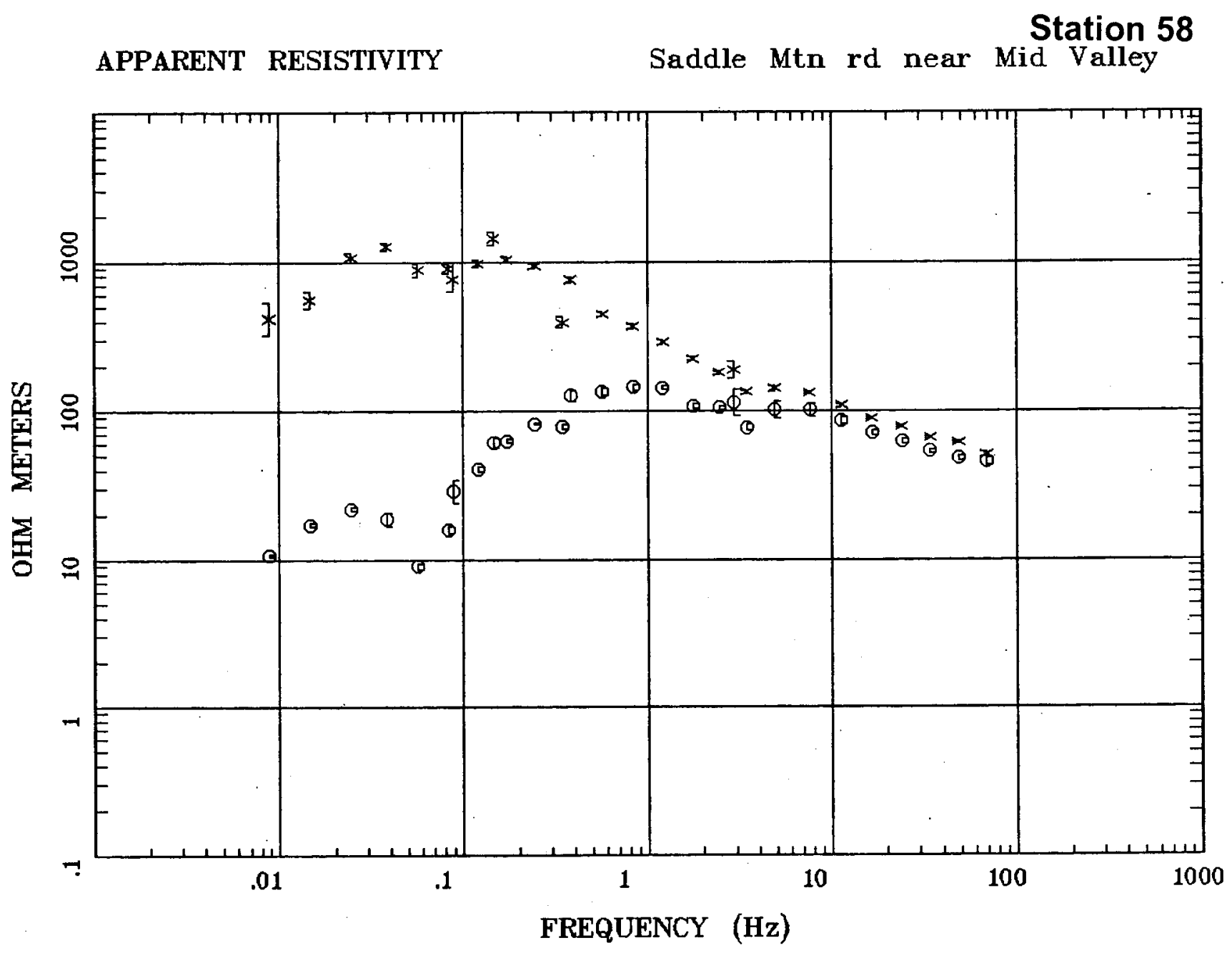

Client: WAter Resources \& DOD Remote: none Acquired: 10:3 oct 14, 2005 Survey Co:US Geological Survey
Rotation:

Filename: nt58m.avg Channels: Ch1 Ch2 Ch3 Ch4 Ch5 Ch3 Ch4 Plotted: 09:43 Jan 30, 2006

< EMI - ElectroMagnetic Instruments > 
IMPEDANCE PHASE

Station 58

Saddle Mtn rd near Mid Valley

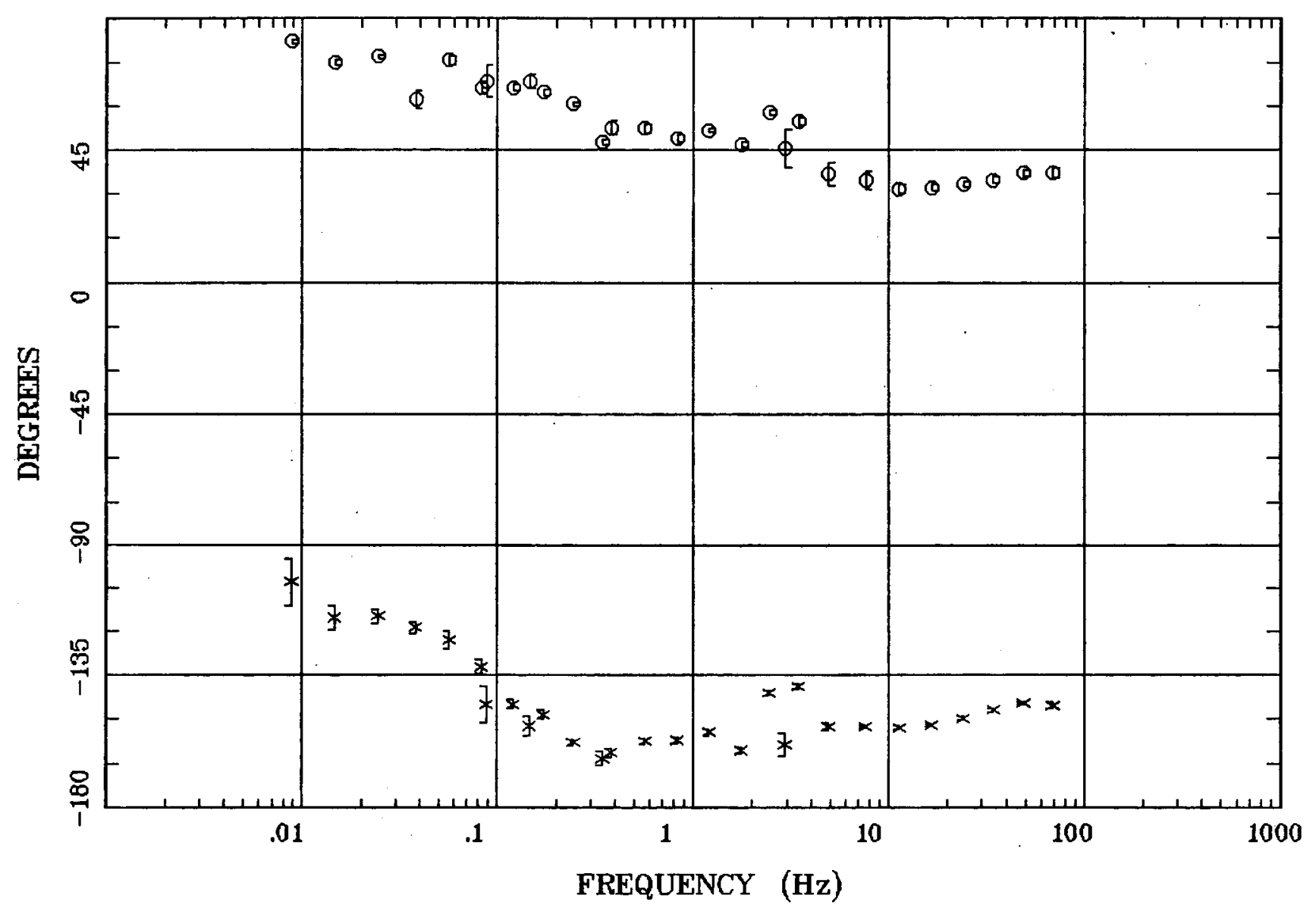

Client: WAter Resources \& DOD Remote: none Acquired: 10:3 Oct 14, 2005 Survey Co:US Geological Survey
Rotation:

Filename: nt58m.arg Channels: Ch1 Ch2 Ch3 Ch4 Ch5 Ch3 Ch4 Plotted: 09:43 Jan 30, 2006

< EMI - ElectroMagnetic Instruments > 


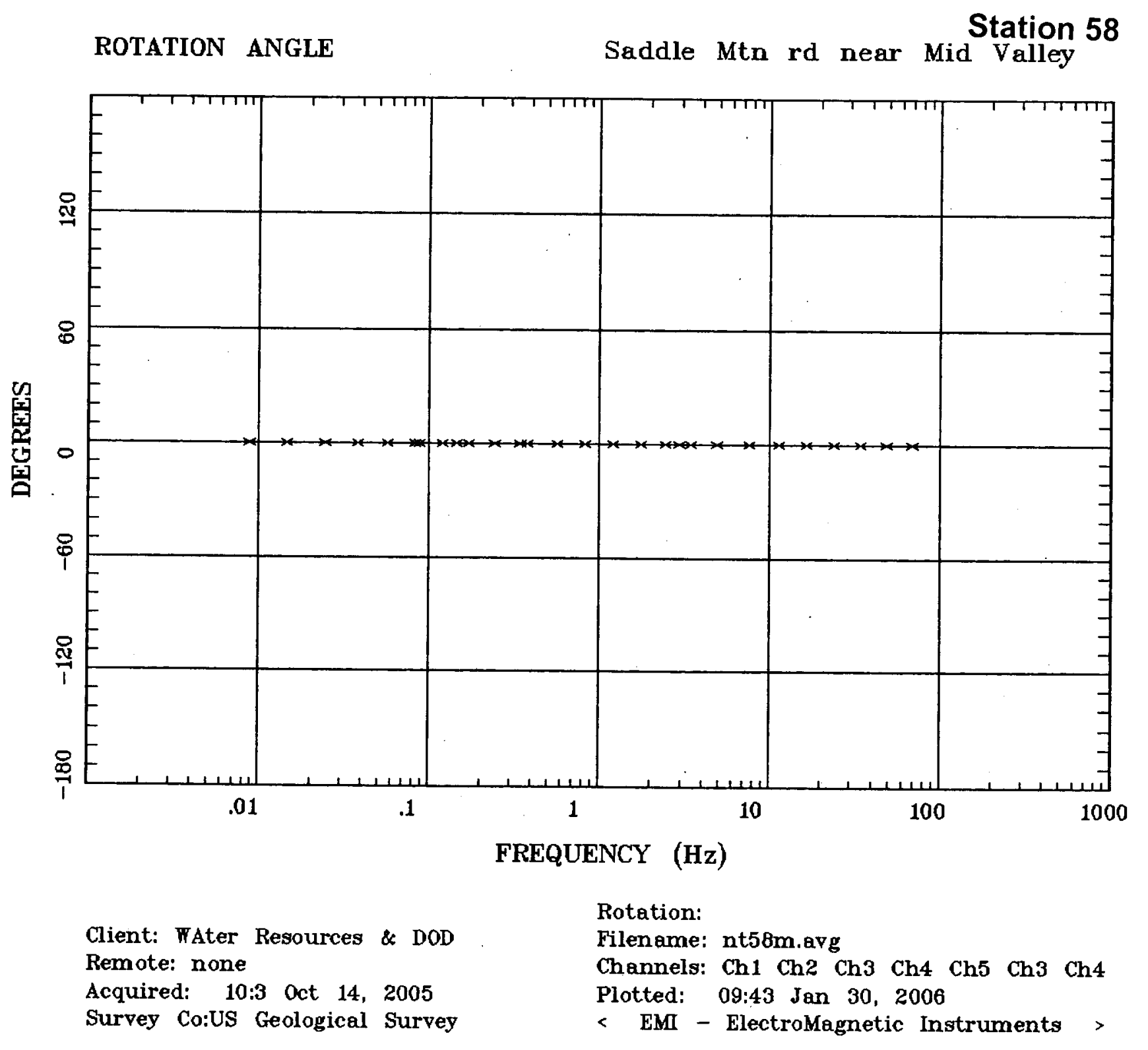


Station 58

IMPEDANCE SKEW

Saddle Mtn rd near Mid Valley

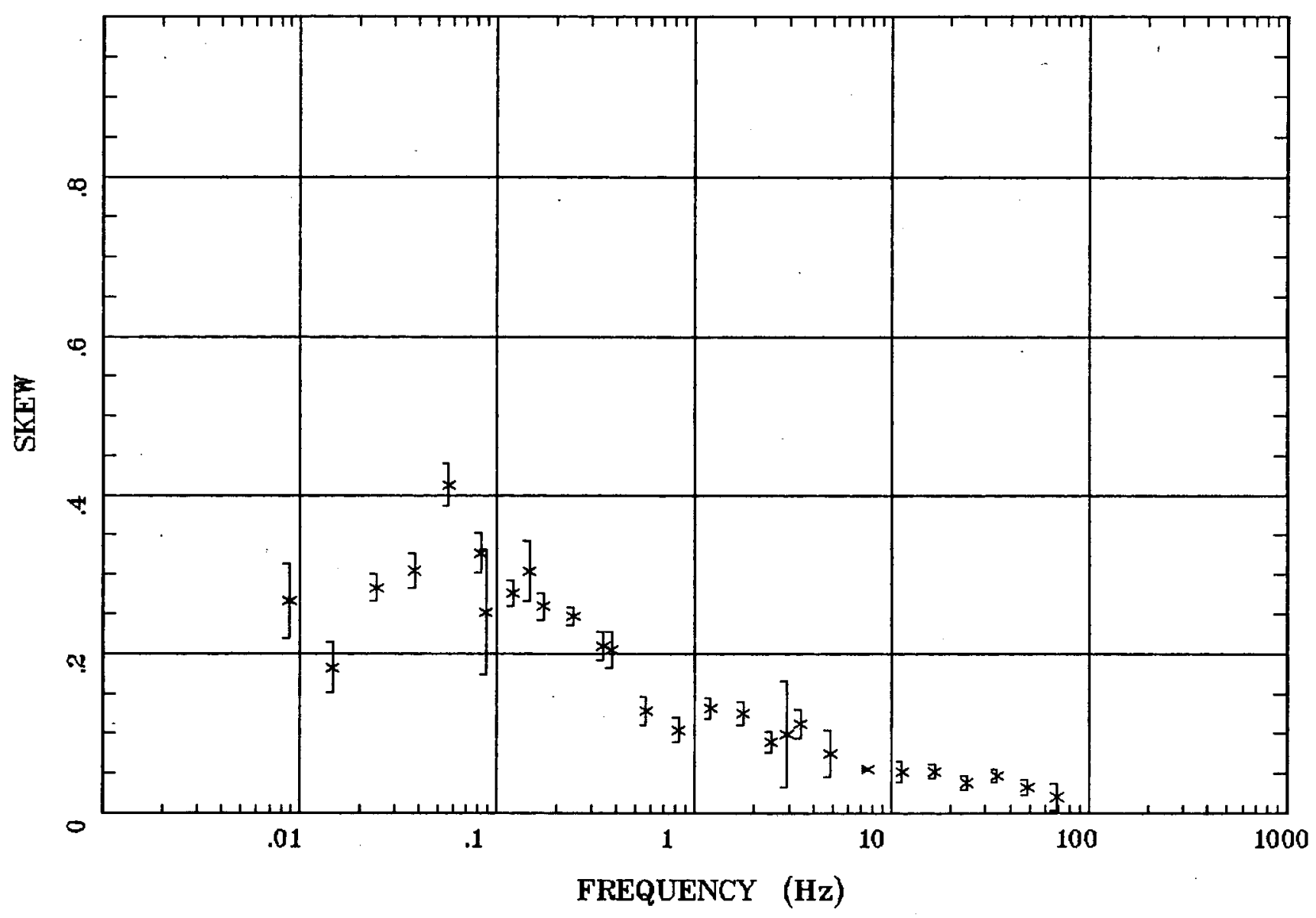

Client: WAter Resources \& DOD

Remote: none

Acquired: $10: 3$ oct 14, 2005

Survey Co:US Geological Survey

Rotation:

Filename: nt58m.avg

Channels: Ch1 Ch2 Ch3 Ch4 Ch5 Ch3 Ch4

Plotted: 09:43 Jan 30, 2006

< EMI - ElectroMagnetic Instruments > 


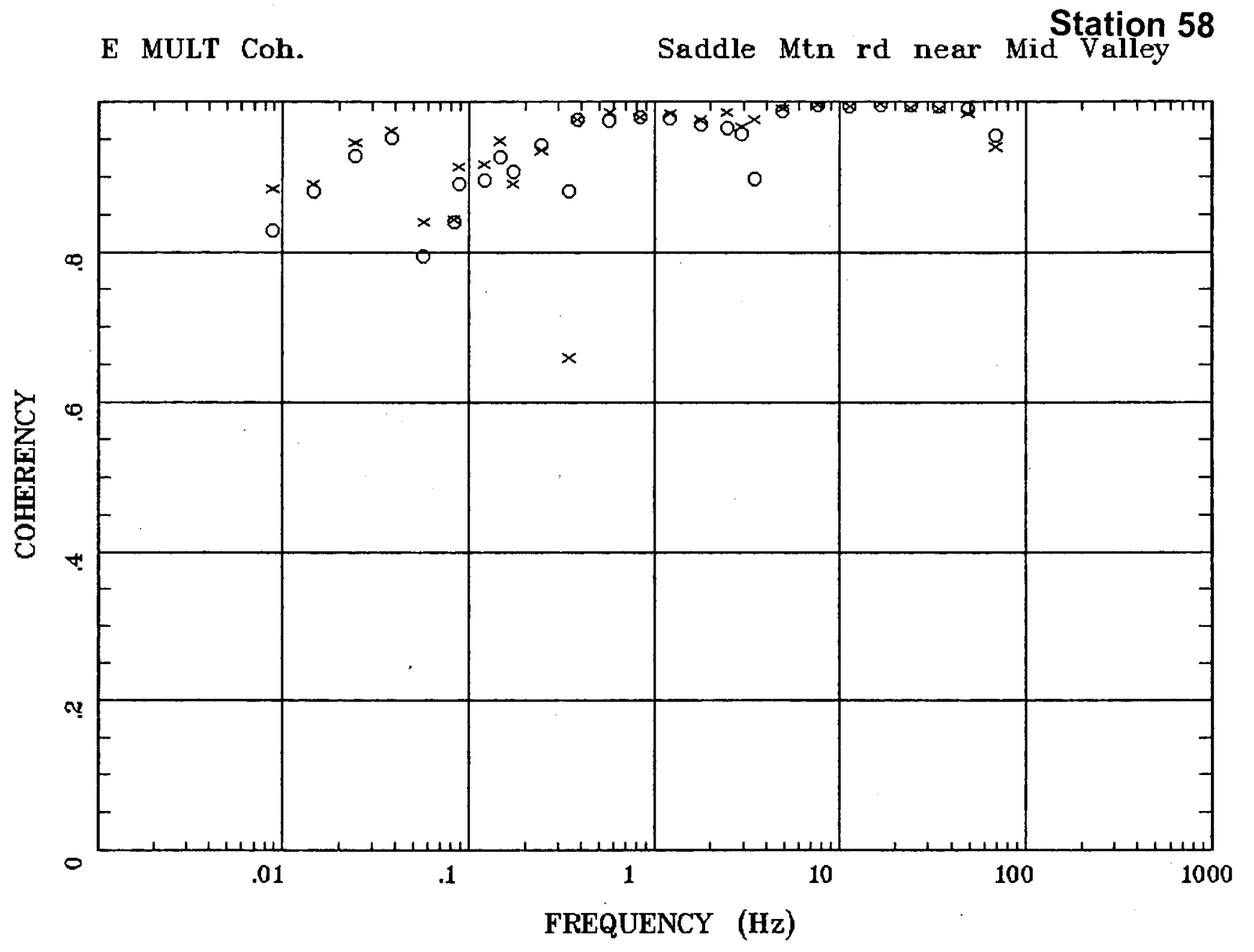

Client: WAter Resources \& DOD Remote: none

Acquired: $10: 3$ oct 14, 2005 Survey Ca:US Geological Survey

Rotation:

Filename: nt58m.arg

Channels: Ch1 Ch2 Ch3 Ch4 Ch5 Ch3 Ch4

Plotted: 09:43 Jan 30, 2006

< EMI - ElectroMagnetic Instruments > 


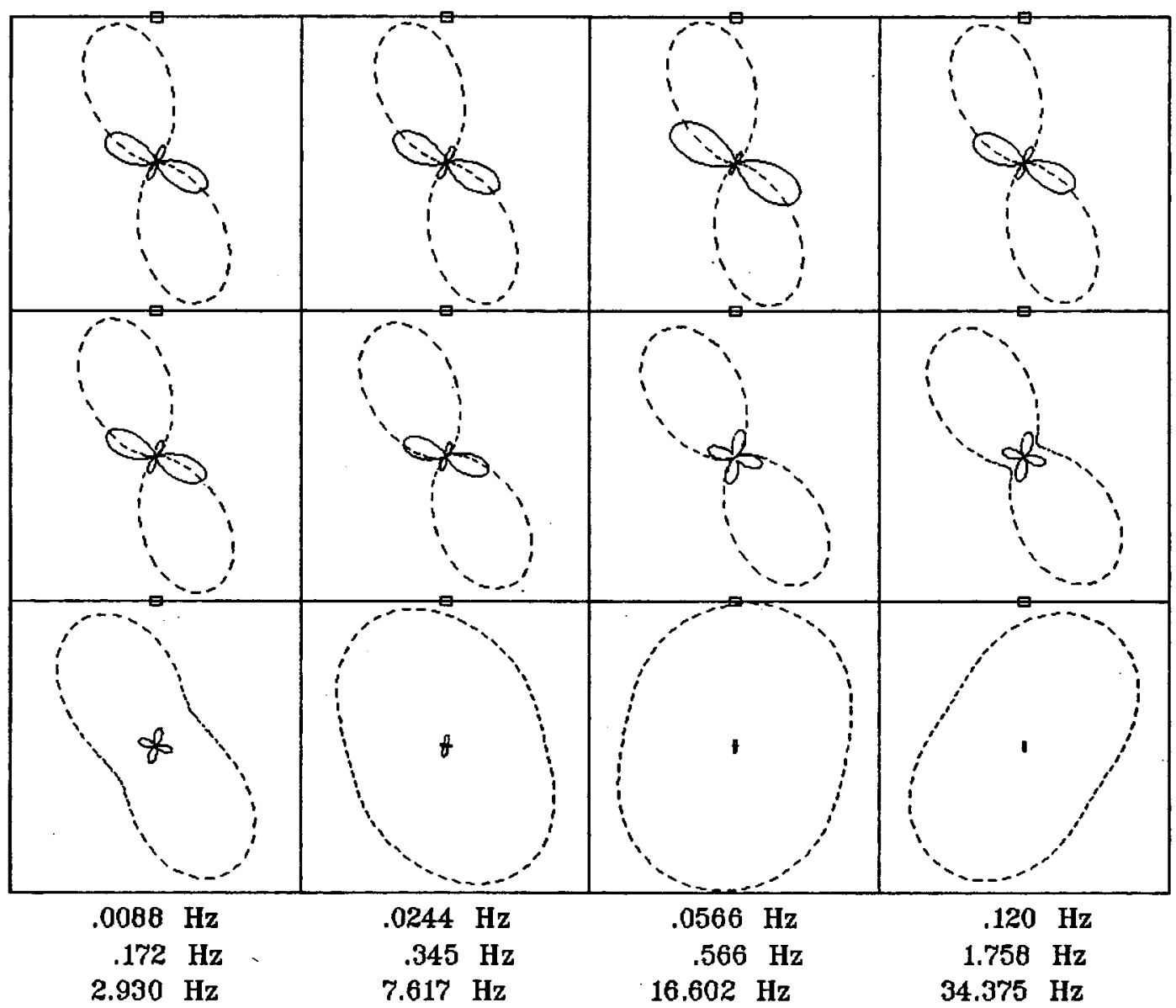

Client: WAter Resources \& DOD Remote: none Acquired: $\quad 10: 3$ oct 14, 2005 Survey Co:US Geological Survey
Rotation:

Filename: nt58m.avg Channels: Ch1 Ch2 Ch3 Ch4 Ch5 Ch3 Ch4 Plotted: 09:43 Jan 30, 2006

< EMI - ElectroMagnetic Instruments > 


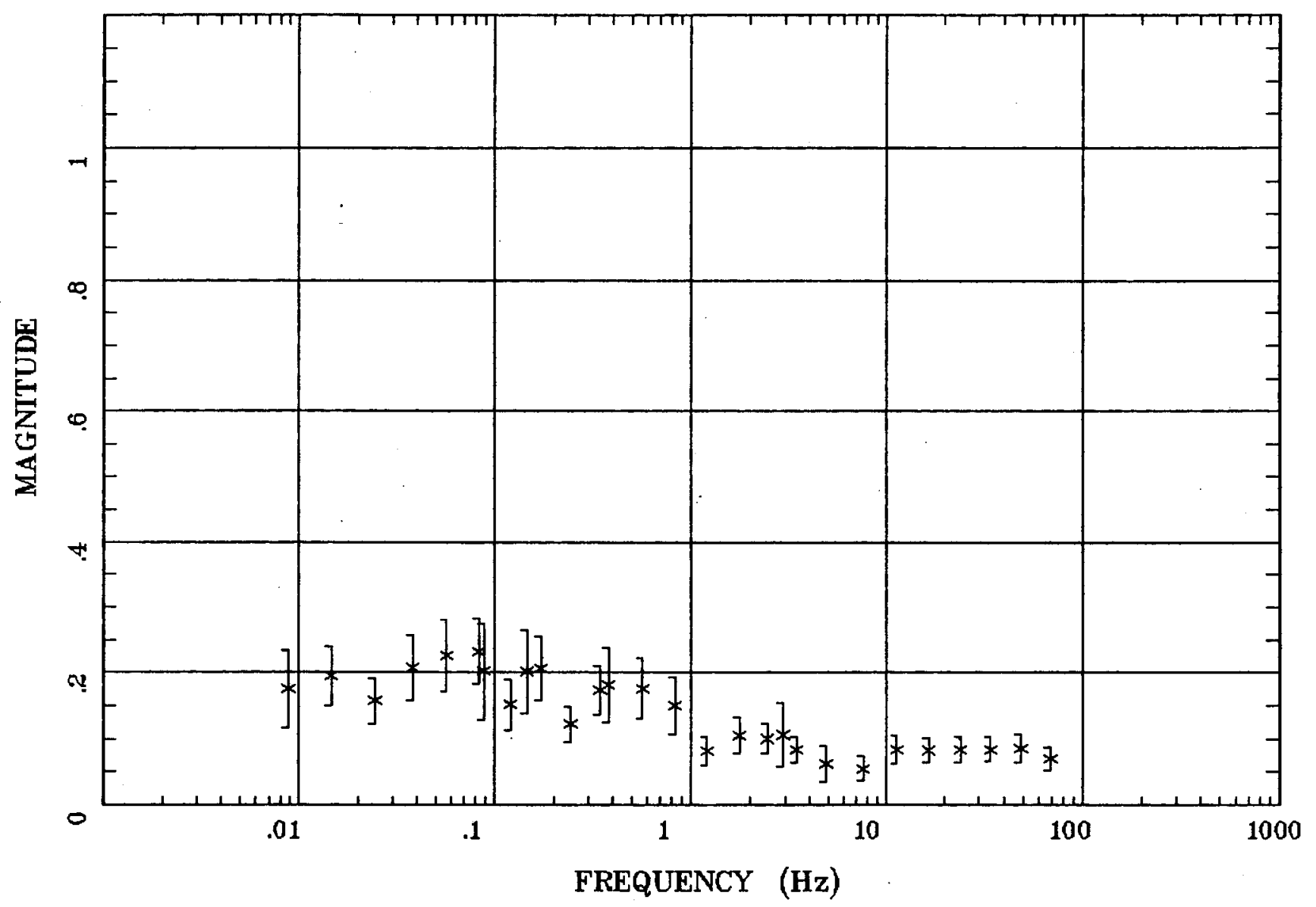

Client: WAter Resources \& DOD Remote: none Acquired: 10:3 Oct 14, 2005 Survey Co:US Geological Survey
Rotation:

Filename: nt58m.avg Channels: Ch1 Ch2 Ch3 Ch4 Ch5 Ch3 Ch4 Plotted: 09:43 Jan 30, 2006

< EMI - ElectroMagnetic Instruments > 
TIPPER STRIKE

Station 58

Saddle Mtn rd near Mid Valley

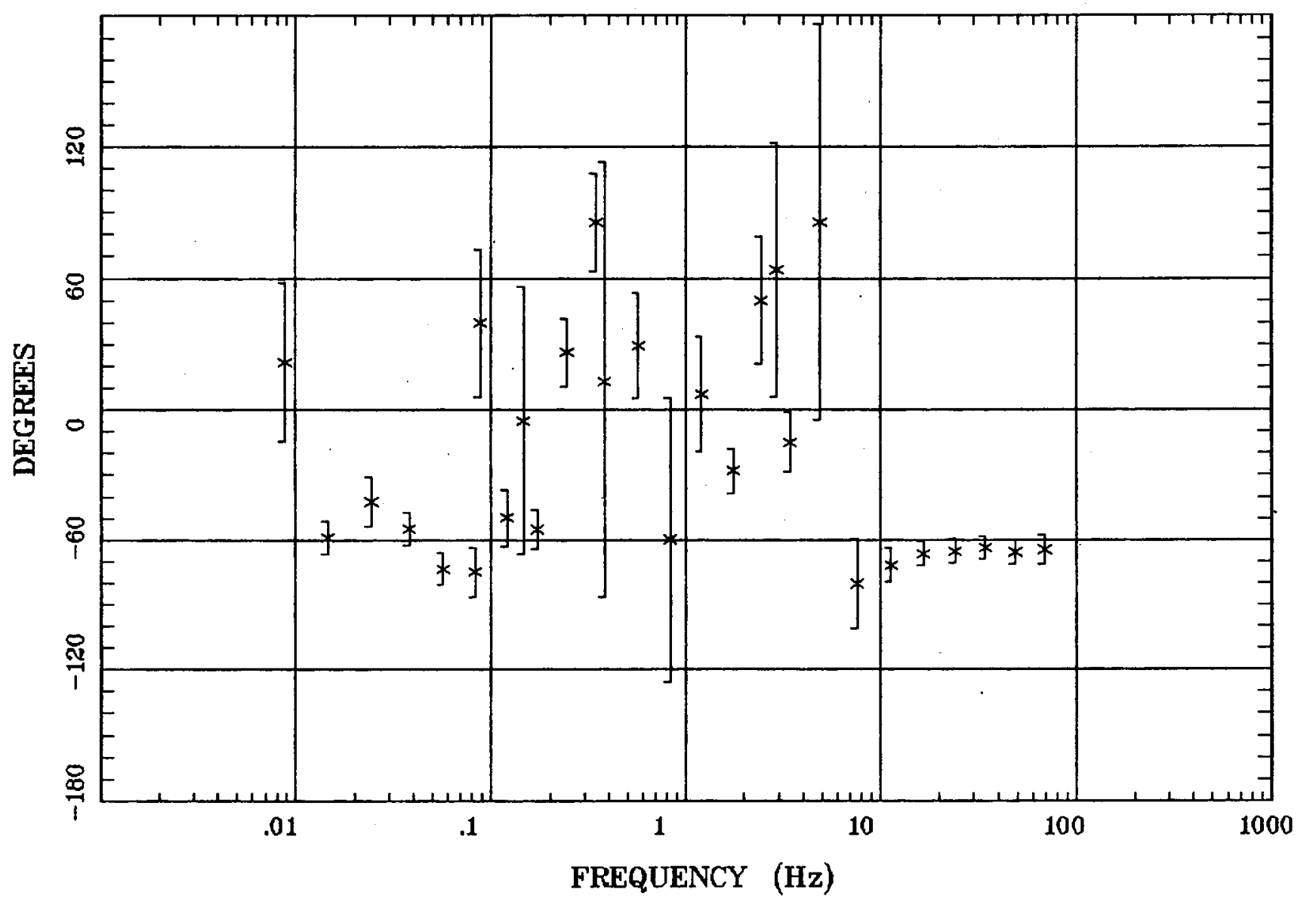

Client: WAter Resources \& DOD

Rotation:

Remote: none

Acquired: 10:3 oct 14, 2005

Survey Co:US Geological Survey

Filename: nt58m.avg

Channels: Ch1 Ch2 Ch3 Ch4 Ch5 Ch3 Ch4

Plotted: 09:43 Jan 30, 2006

< EMI - ElectroMagnetic Instruments > 
HzHx.x Coh HzHy.o

Station 58

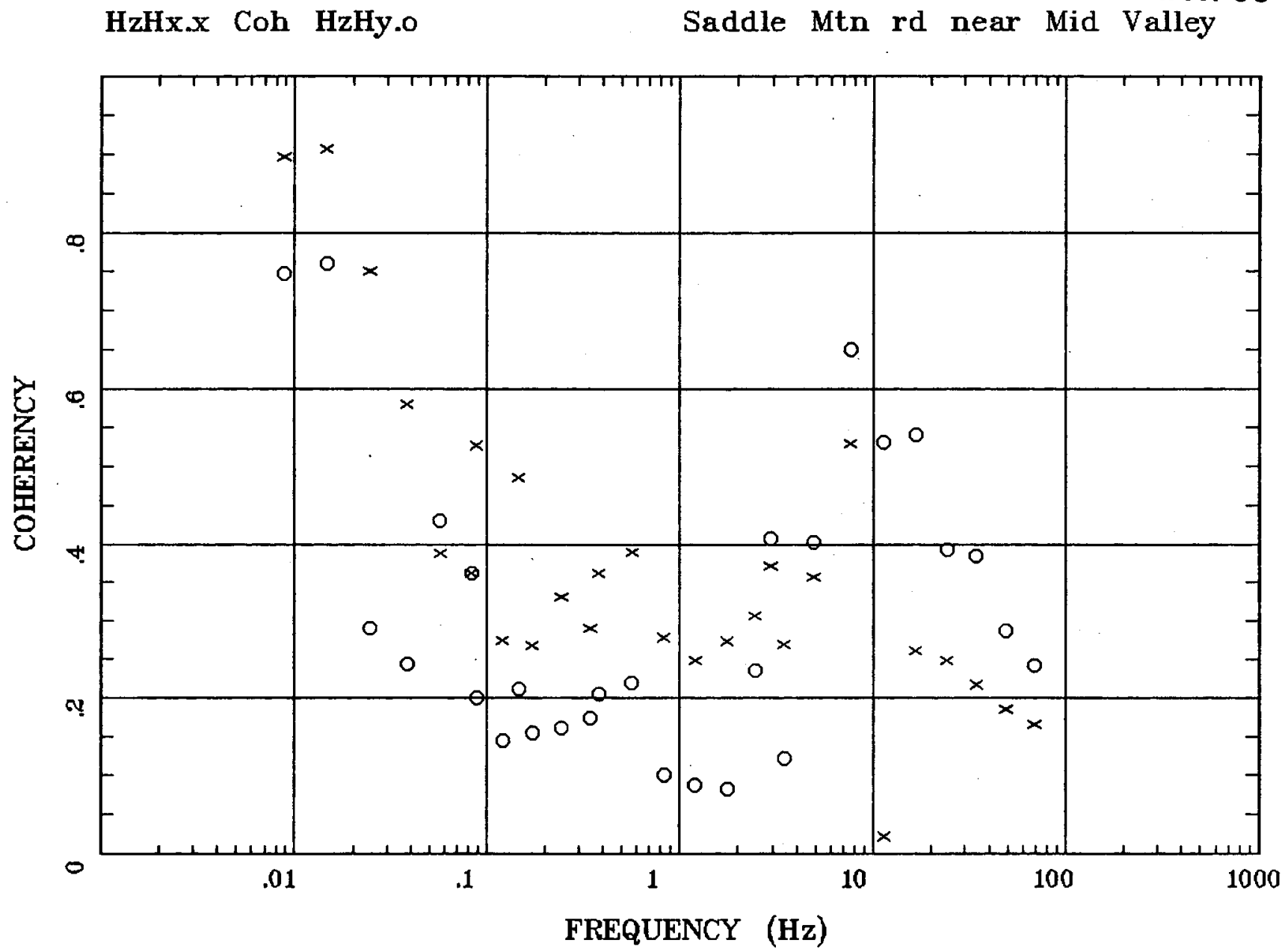

Client: WAter Resources \& DOD Remote: none

Acquired: 10:3 Oct 14, 2005 Survey Co:US Geological Survey
Rotation:

Filename: nt58m.avg

Channels: Ch1 Ch2 Ch3 Ch4 Ch5 Ch3 Ch4 Plotted: 09:43 Jan 30, 2006

< EMI - ElectroMagnetic Instruments > 
Station 59

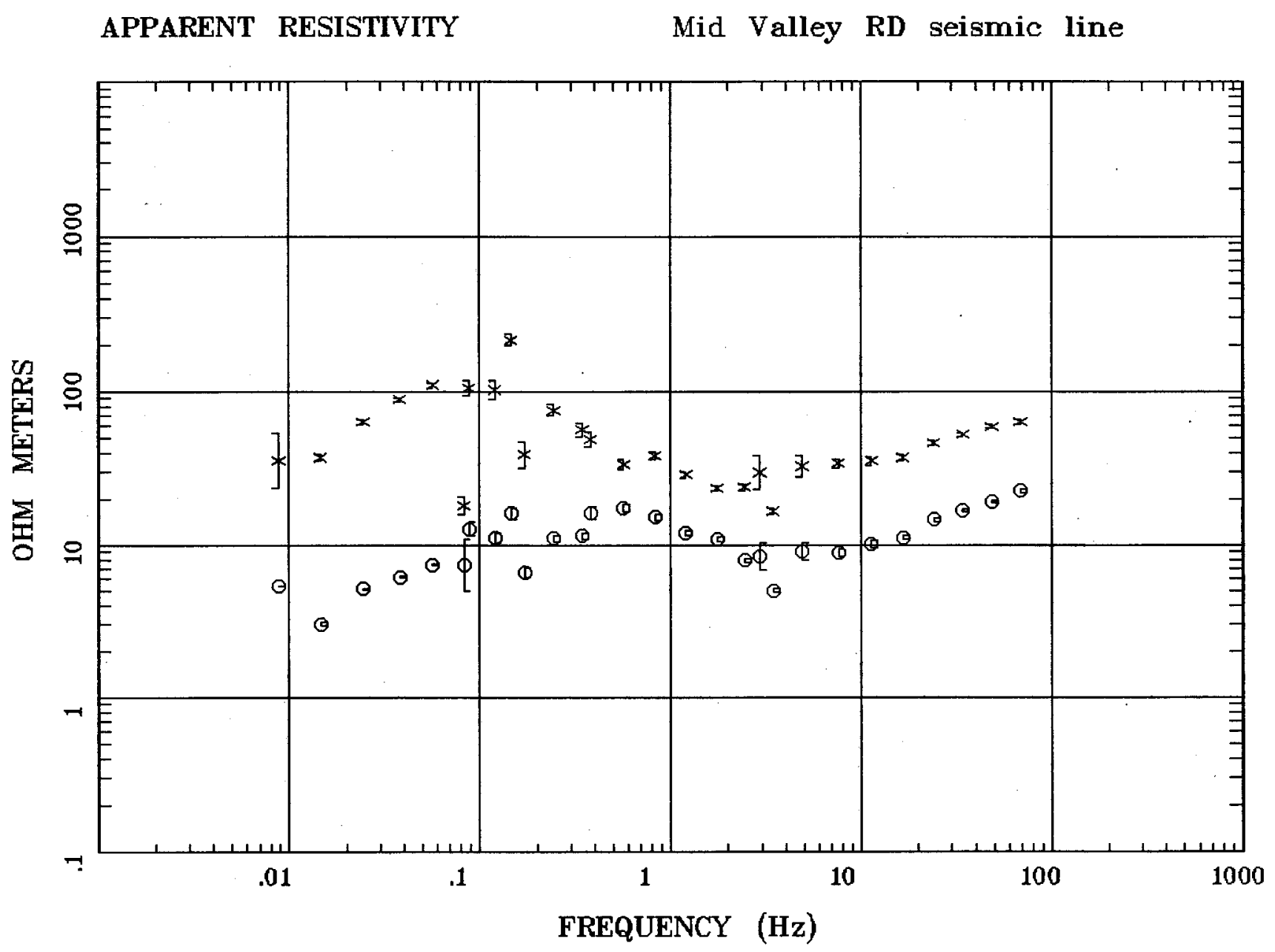

Client: WAter Resources \& DOD Remote: none

Acquired: 04:3 oct 15, 2005 Survey Co:US Geological Survey
Rotation:

Filename: nt59m.avg

Channels: Ch1 Ch2 Ch3 Ch4 Ch5 Ch3 Ch4 Plotted: 14:58 Feb 08, 2006

< EMI - ElectroMagnetic Instruments > 
IMPEDANCE PHASE

Mid Valley RD seismic line

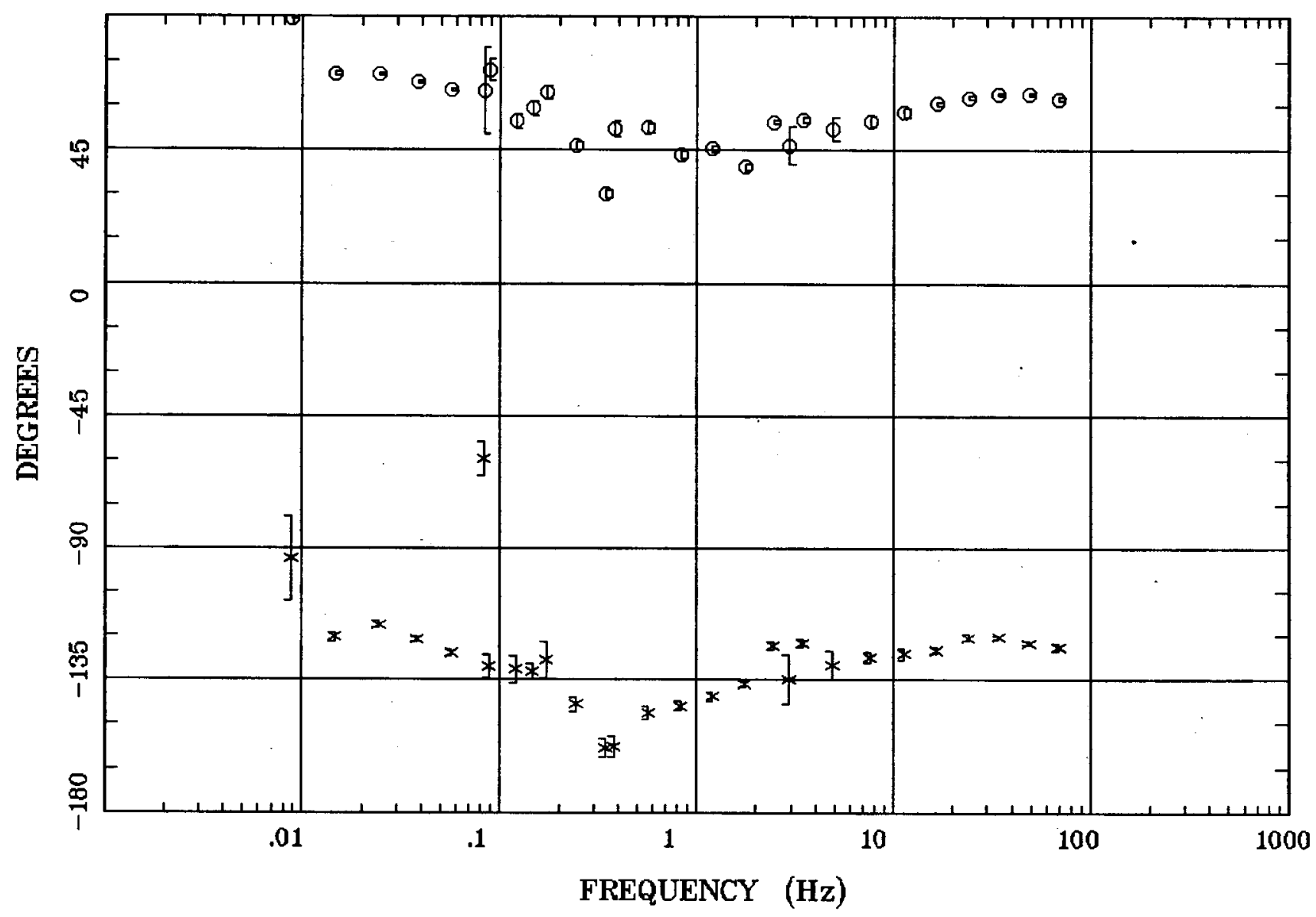

Client: WAter Resources \& DOD Remote: none

Acquired: 04:3 oct 15, 2005 Survey Co:US Geological Survey
Rotation:

Filename: nt59m.avg Channels: Ch1 Ch2 Ch3 Ch4 Ch5 Ch3 Ch4 Plotted: 14:58 Feb 08, 2006

< EMI - ElectroMagnetic Instruments > 
ROTATION ANGLE

Station 59

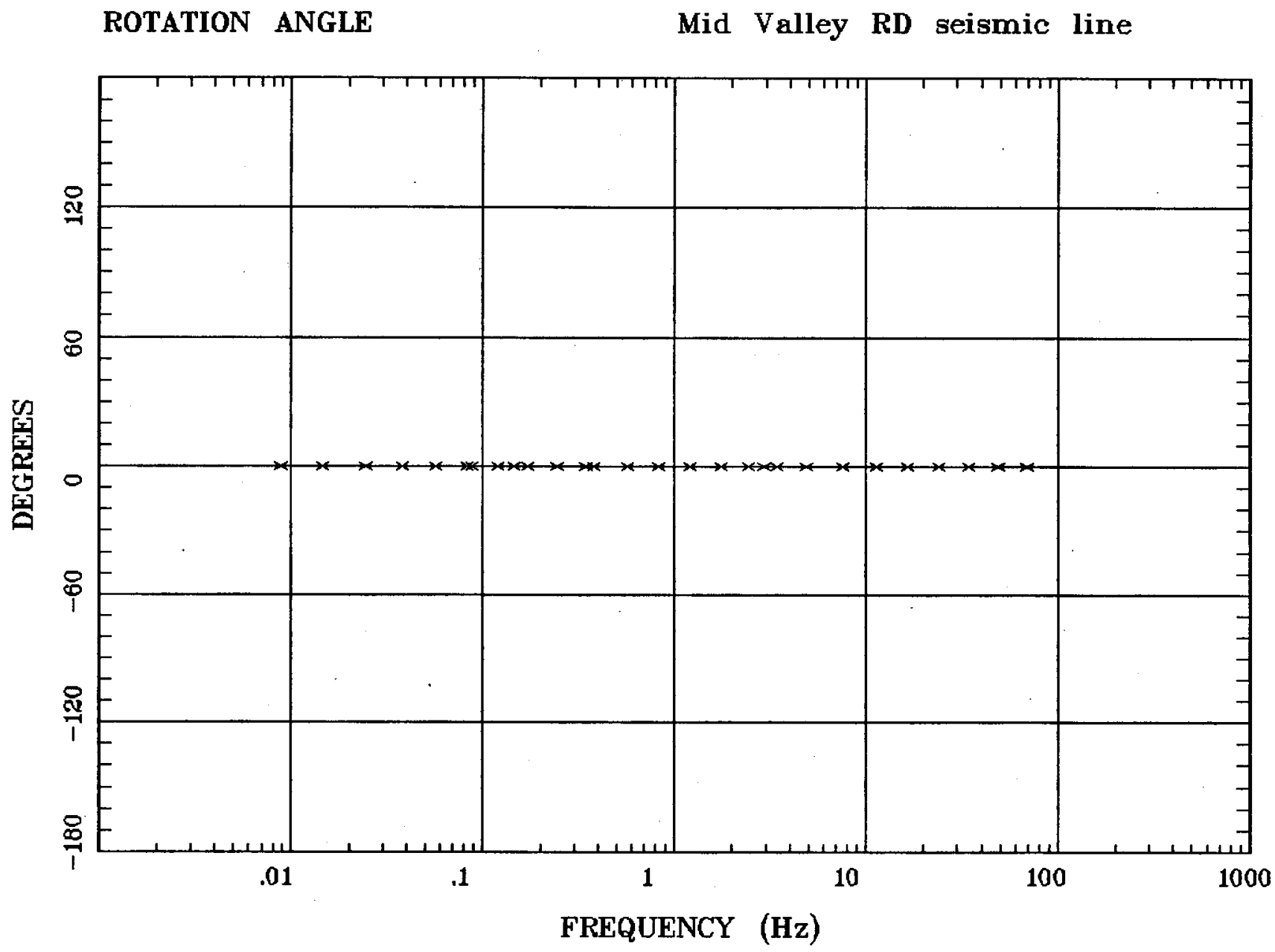

Client: WAter Resources \& DOD Remote: none

Acquired: 04:3 Oct 15, 2005

Survey Co:US Geological Survey

Rotation:

Filename: nt59m.avg

Channels: Ch1 Ch2 Ch3 Ch4 Ch5 Ch3 Ch4

Plotted: 14:58 Feb 08, 2006

< EMI - ElectroMagnetic Instruments > 


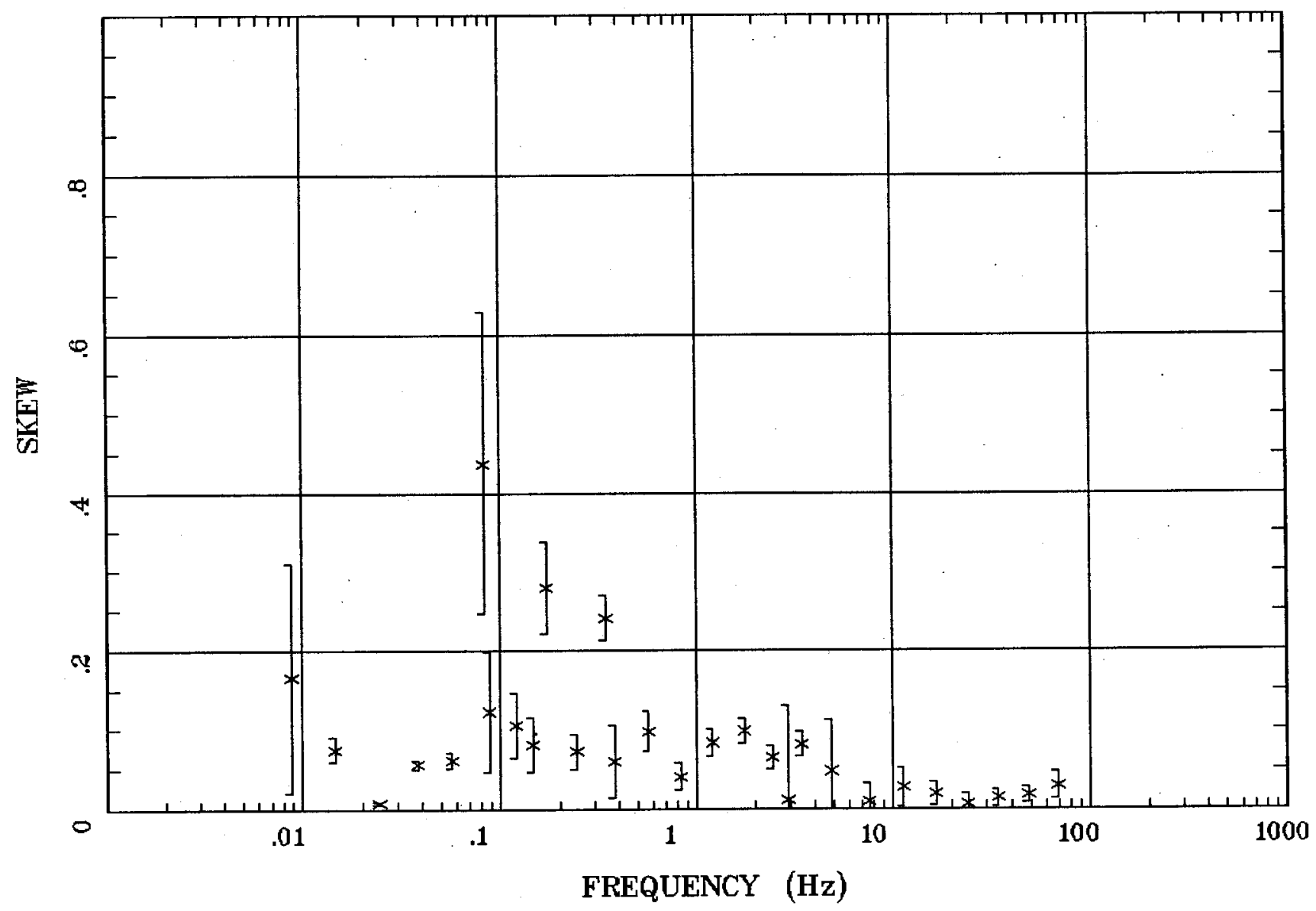

Client: WAter Resources \& DOD Remote: none Acquired: $04: 3$ oct 15,2005 Survey Co:US Geological Survey
Rotation:

Filename: nt59m.avg Channels: Ch1 Ch2 Ch3 Ch4 Ch5 Ch3 Ch4 Plotted: 14:58 Feb 08, 2006

< EMI - ElectroMagnetic Instruments > 
Station 59

E MULT Coh.

Mid Valley RD seismic line

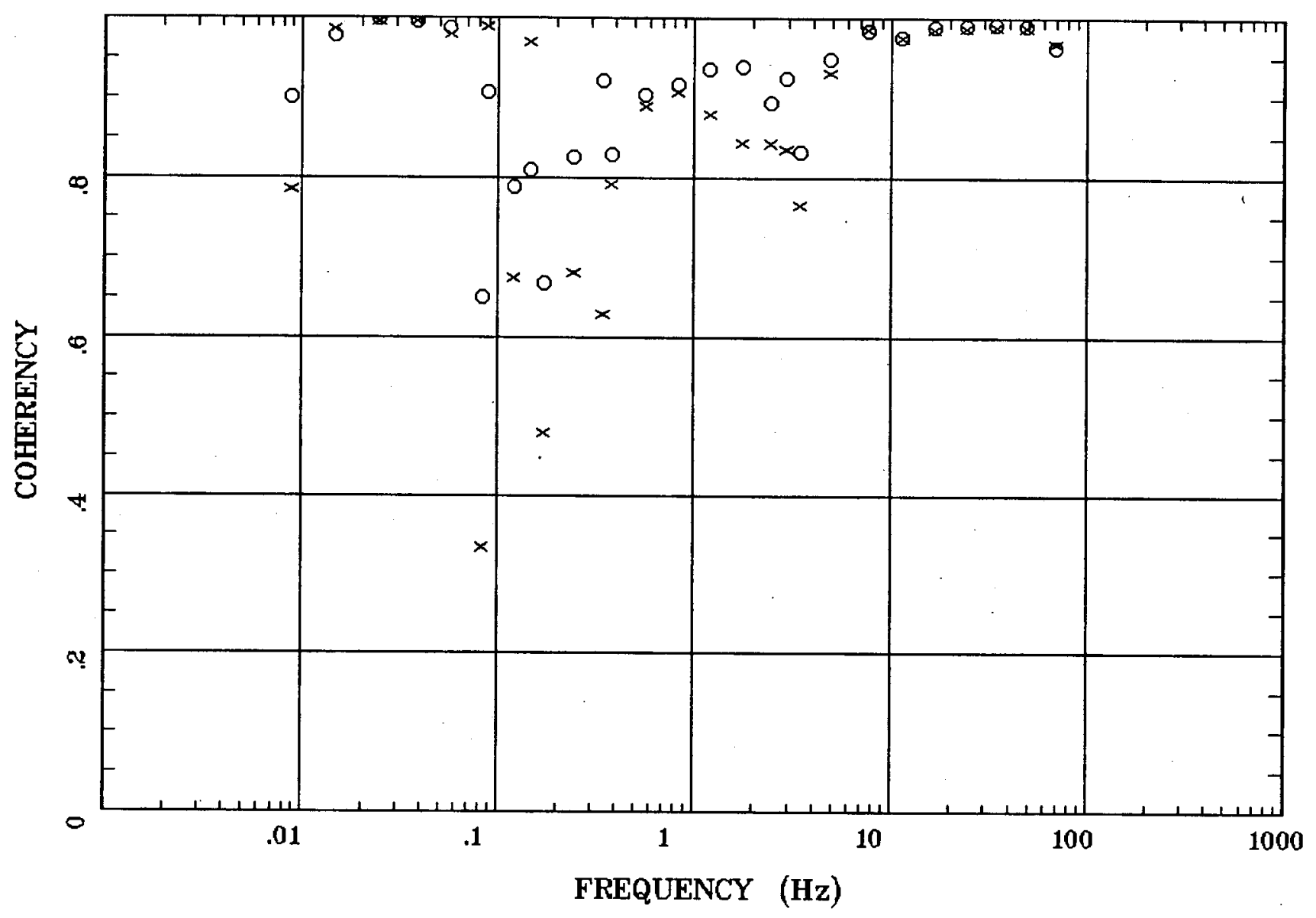

Client: FAter Resources \& DOD Remote: none

Acquired: 04:3 Oct 15, 2005 Survey Co:US Geological Survey
Rotation:

Filename: nt59m.avg Channels: Ch1 Ch2 Ch3 Ch4 Ch5 Ch3 Ch4 Plotted: 14:58 Feb 08, 2006

< EMI - ElectroMagnetic Instruments > 
Station 59

POLAR PLOTS

Mid Valley RD seismic line

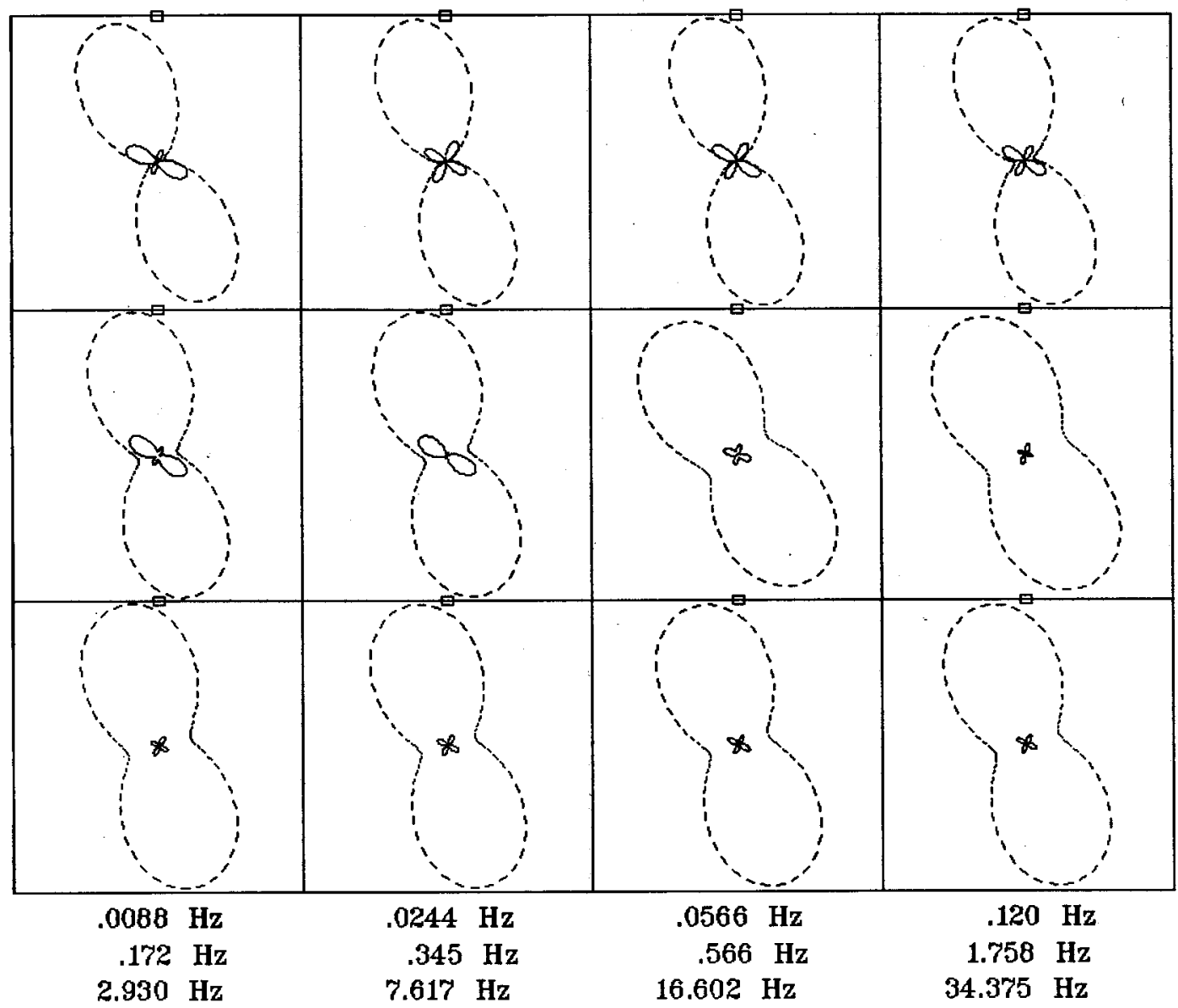

Rotation:

Client: WAter Resources \& DOD Remote: none Acquired: 04:3 oct 15, 2005 Survey Co:US Geological Survey

Filename: nt59m.avg Channels: Ch1 Ch2 Ch3 Ch4 Ch5 Ch3 Ch4

Plotted: 14:58 Feb 08, 2006

< EMI - ElectroMagnetic Instruments > 
Mid Valley $R D$ seismic line

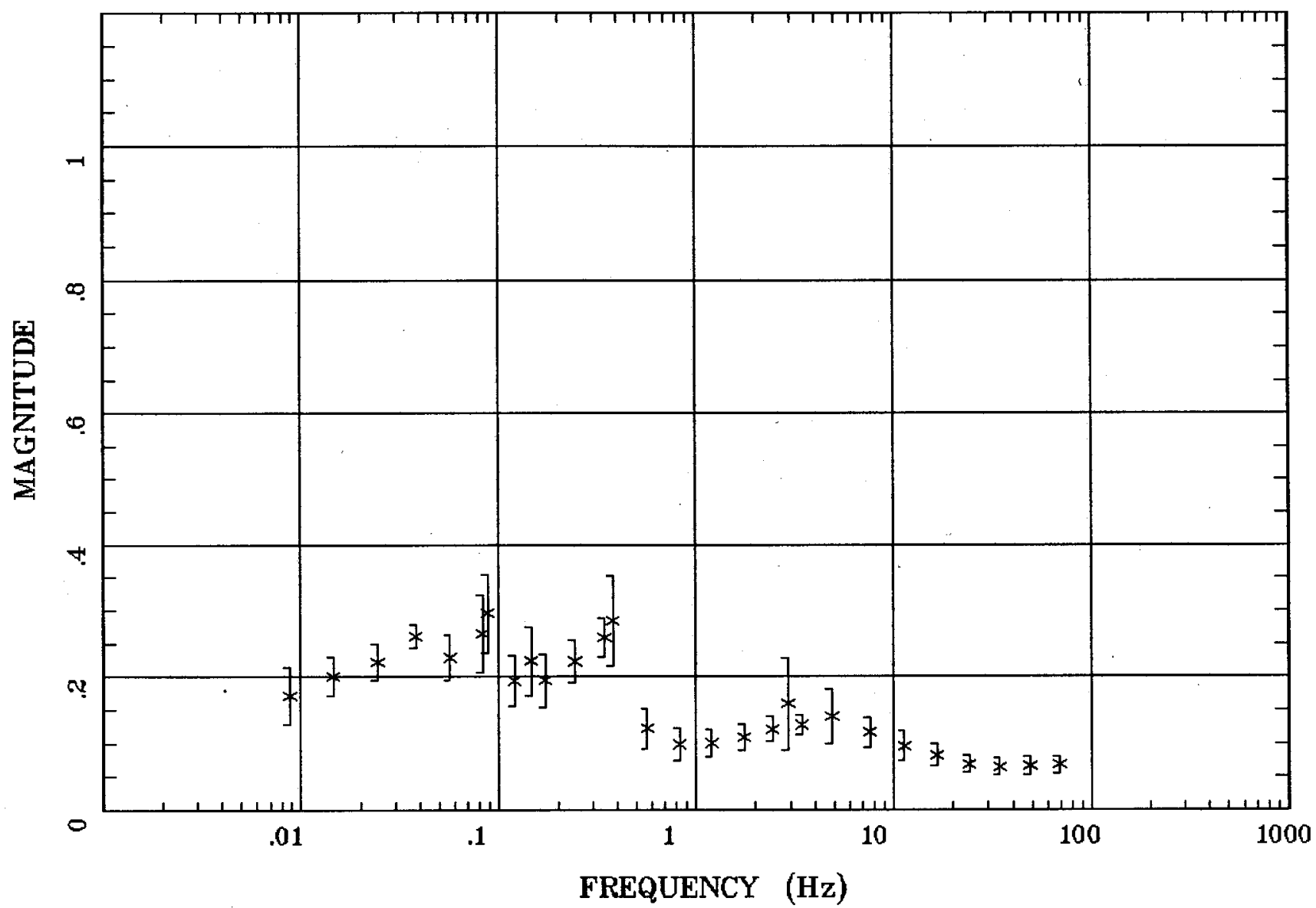

Client: WAter Resources \& DOD Remote: none

Acquired: 04:3 oct 15, 2005 Survey Ca:US Geological Survey
Rotation:

Filename: nt59m.avg Channels: Ch1 Ch2 Ch3 Ch4 Ch5 Ch3 Ch4 Plotted: 14:58 Feb 08, 2006

< EMI - ElectroMagnetic Instruments > 
Station 59

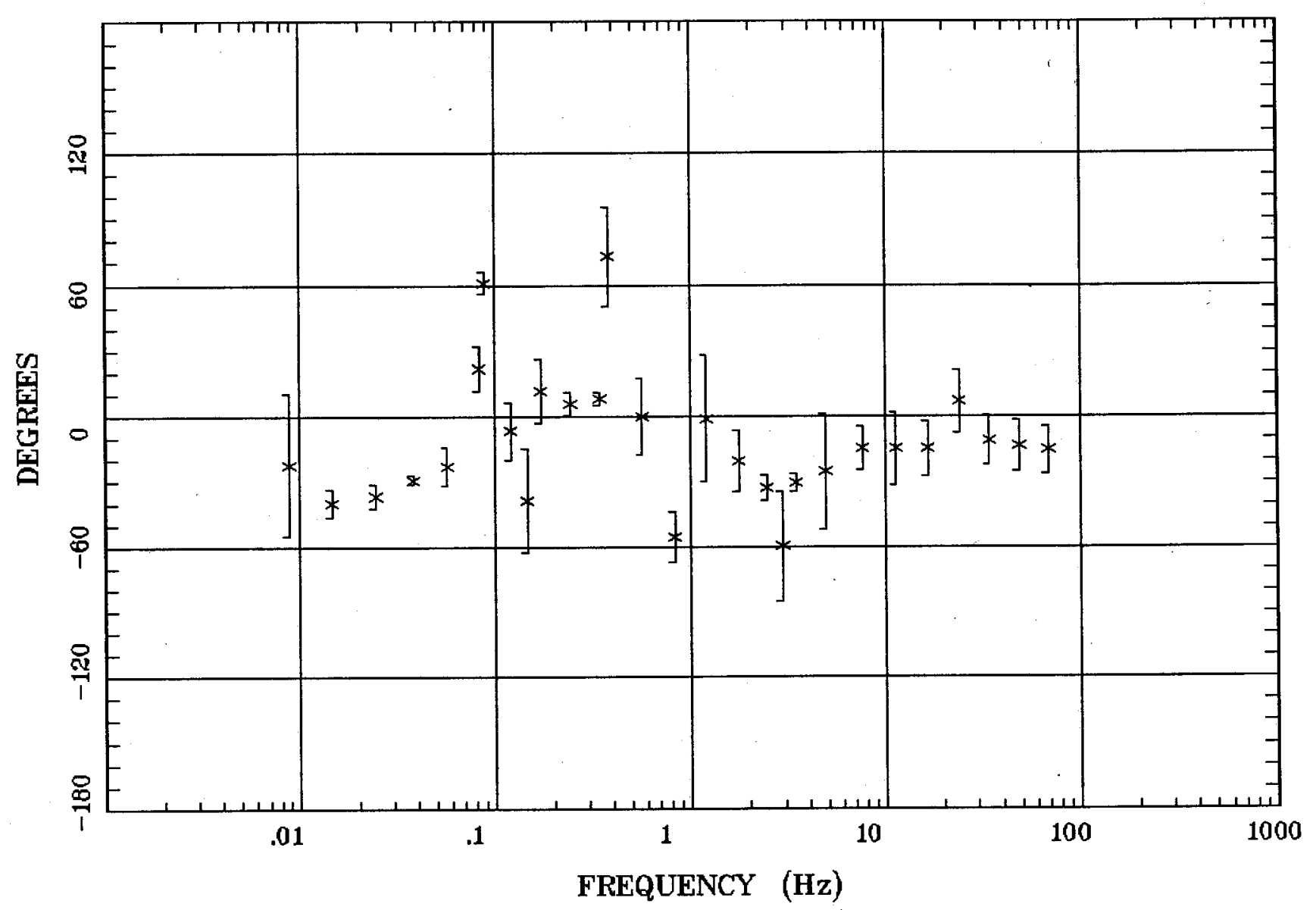

Client: WAter Resources \& DOD Remote: none Acquired: 04:3 oct 15, 2005 Survey Co:US Geological Survey

Mid Valley RD seismic line 
HzHx.x Coh HzHy.o

Station 59

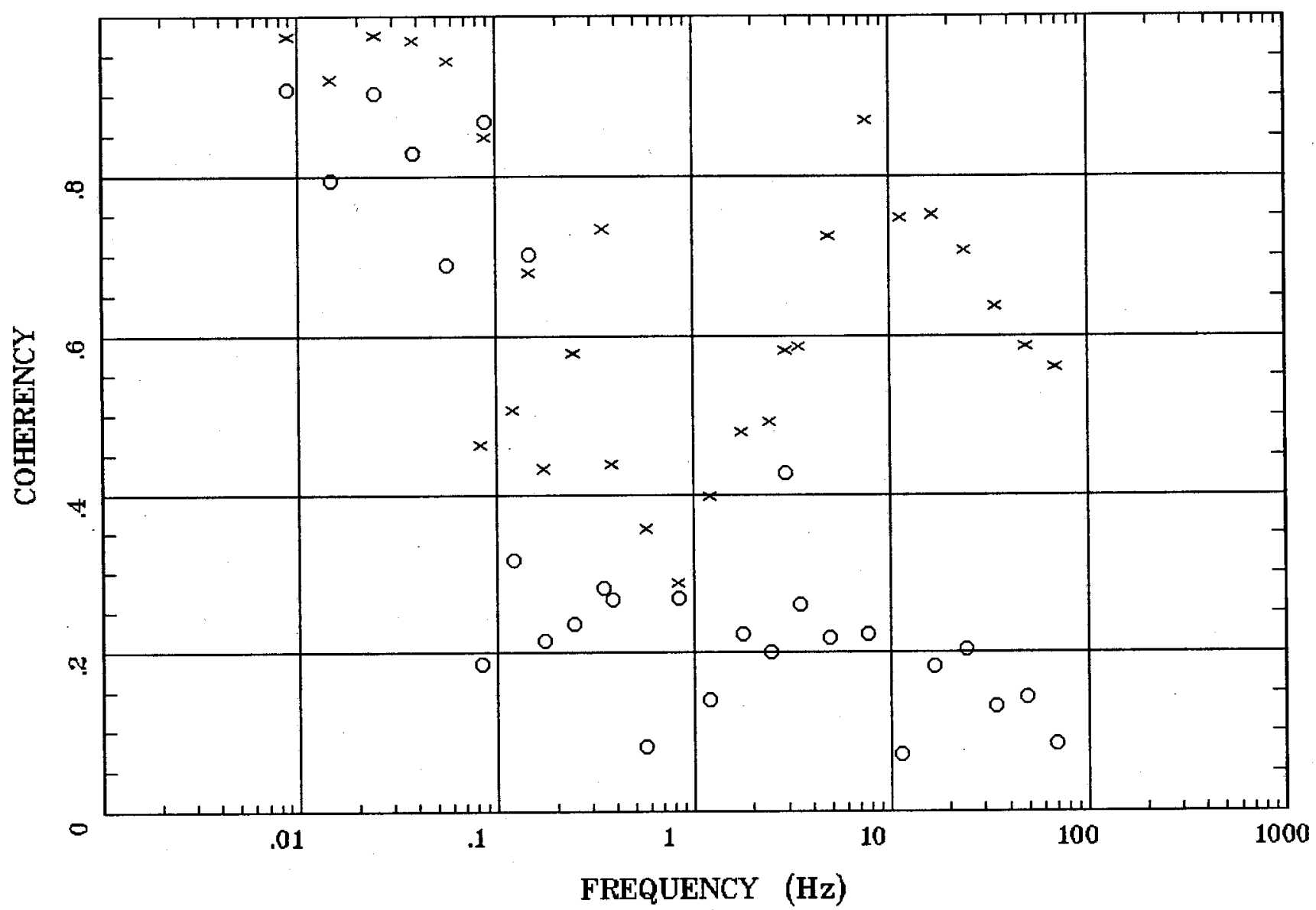

Client: WAter Resources \& DOD

Rotation:

Filename: nt59m.avg

Channels: Ch1 Ch2 Ch3 Ch4 Ch5 Ch3 Ch4

Plotted: 14:58 Feb 08, 2006

Acquired: 04:3 oct 15, 2005

Survey Co:US Geological Survey

< EMI - ElectroMagnetic Instruments > 
Station 60

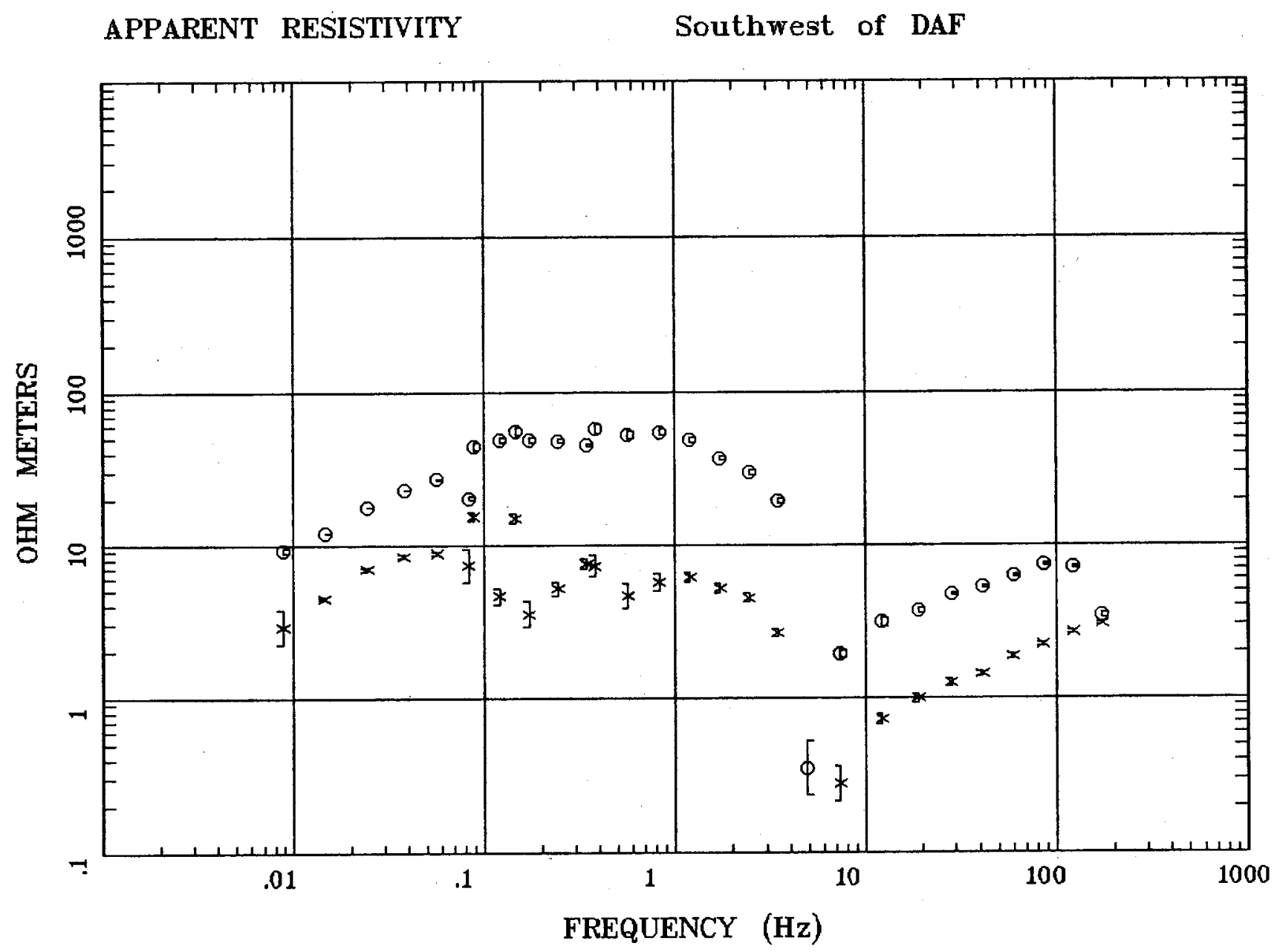

Client: WAter Resources \& DOD Remote: none

Acquired: 11:5 Feb 02, 2006 Survey Co:US Geological Survey
Rotation:

Filename: mt60n.avg Channels: Ch1 Ch2 Ch3 Ch4 Ch5 Ch3 Ch4 Plotted: 12:00 Feb 02, 2006

< EMI - ElectroMagnetic Instruments > 
Station 60

IMPEDANCE PHASE

Southwest of DAF

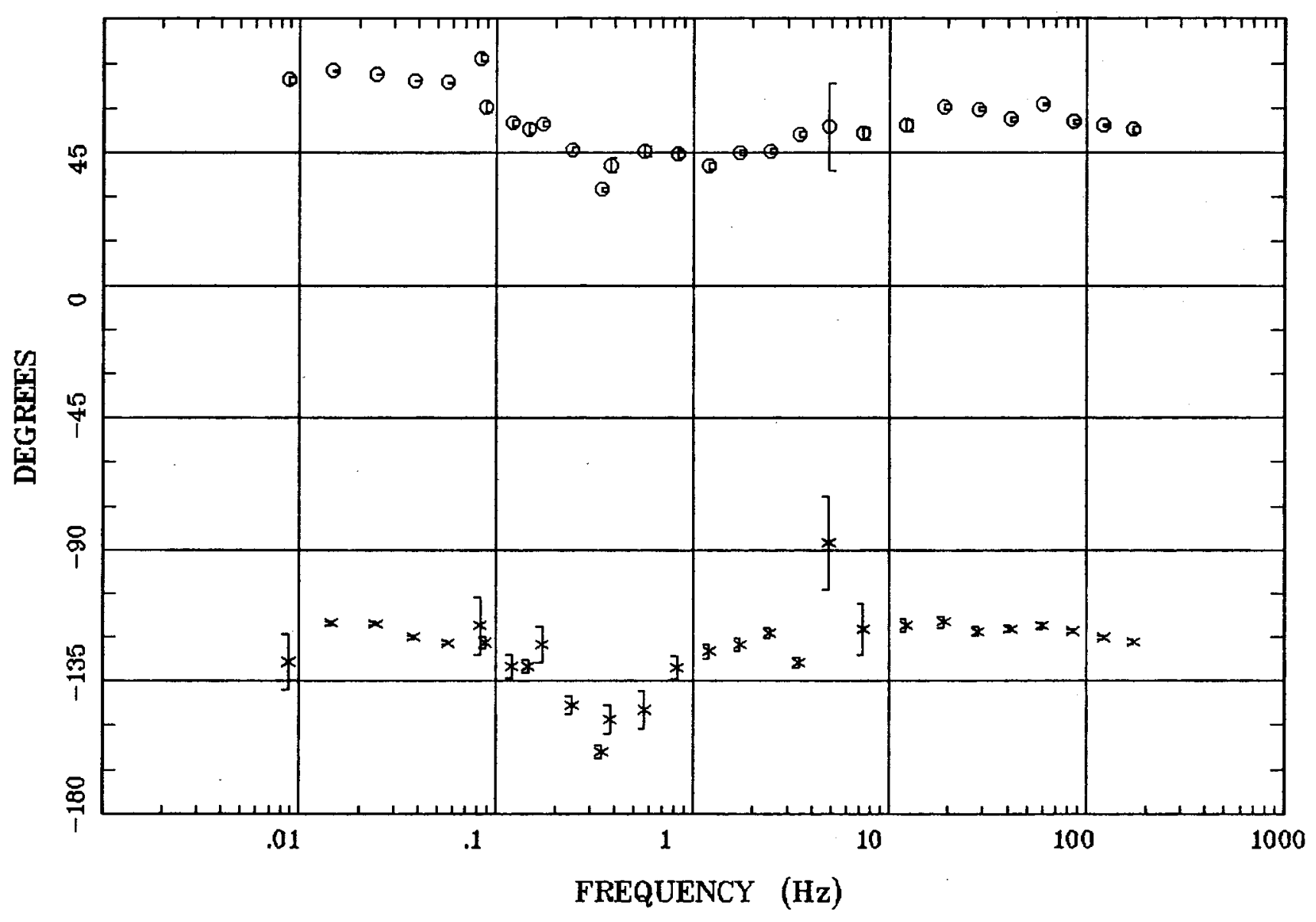

Client: FAter Resources \& DOD

Rotation:

Remote: none

Filename: mt60n.avg

Channels: Ch1 Ch2 Ch3 Ch4 Ch5 Ch3 Ch4

Plotted: 12:00 Feb 02, 2006

Acquired: $11: 5 \mathrm{Feb}$ 02, 2006

Survey Co:US Geological Survey

< EMI - ElectroMagnetic Instruments > 
Station 60

ROTATION ANGLE Southwest of DAF

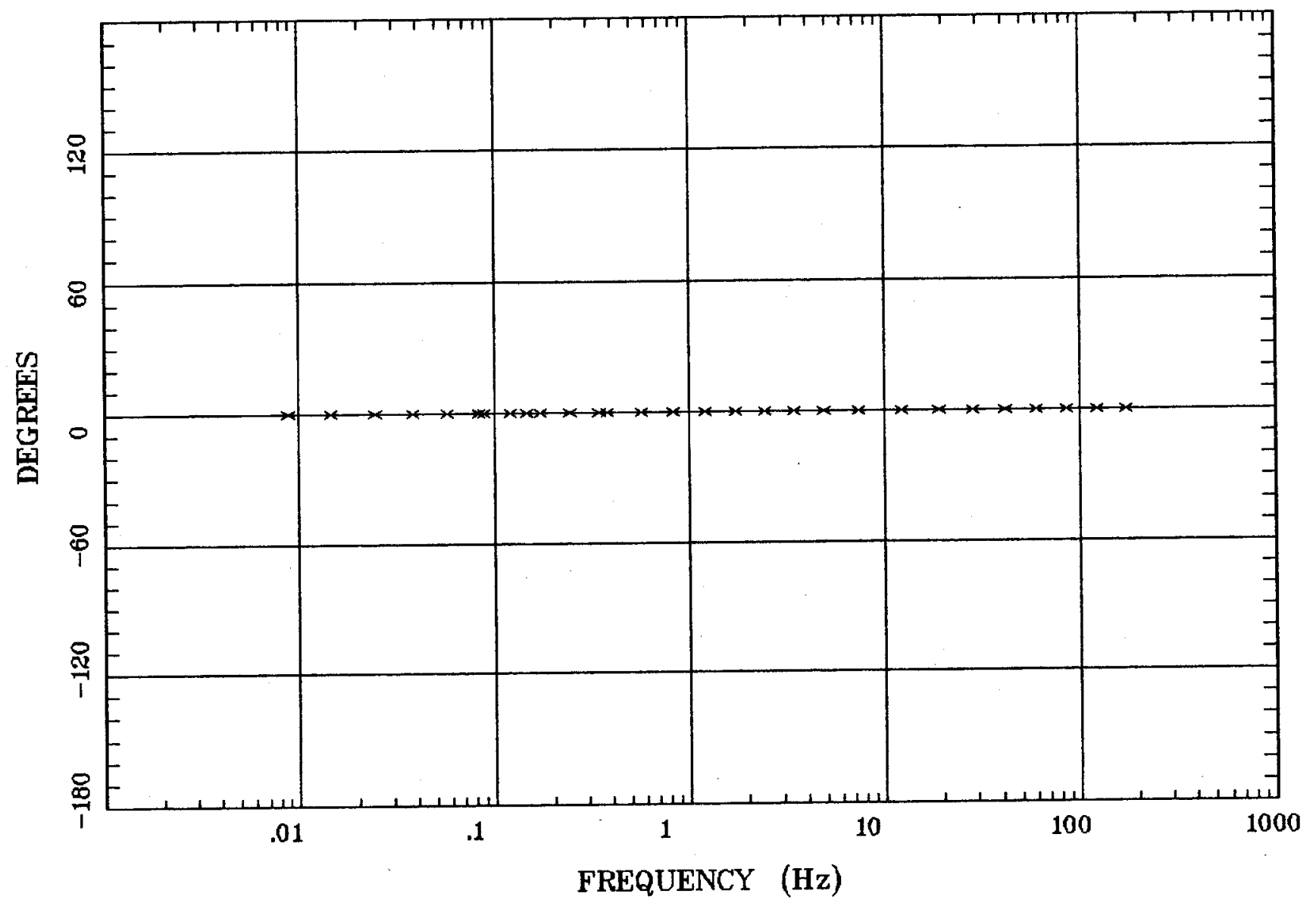

Client: WAter Resources \& DOD

Rotation:

Remote: none

Filename: mt60n.avg

Channels: Ch1 Ch2 Ch3 Ch4 Ch5 Ch3 Ch4

Plotted: 12:00 Feb 02, 2006

Acquired: 11:5 Feb 02, 2006

< EMI - ElectroMagnetic Instruments > 
Station 60

IMPEDANCE SKEW

Southwest of DAF

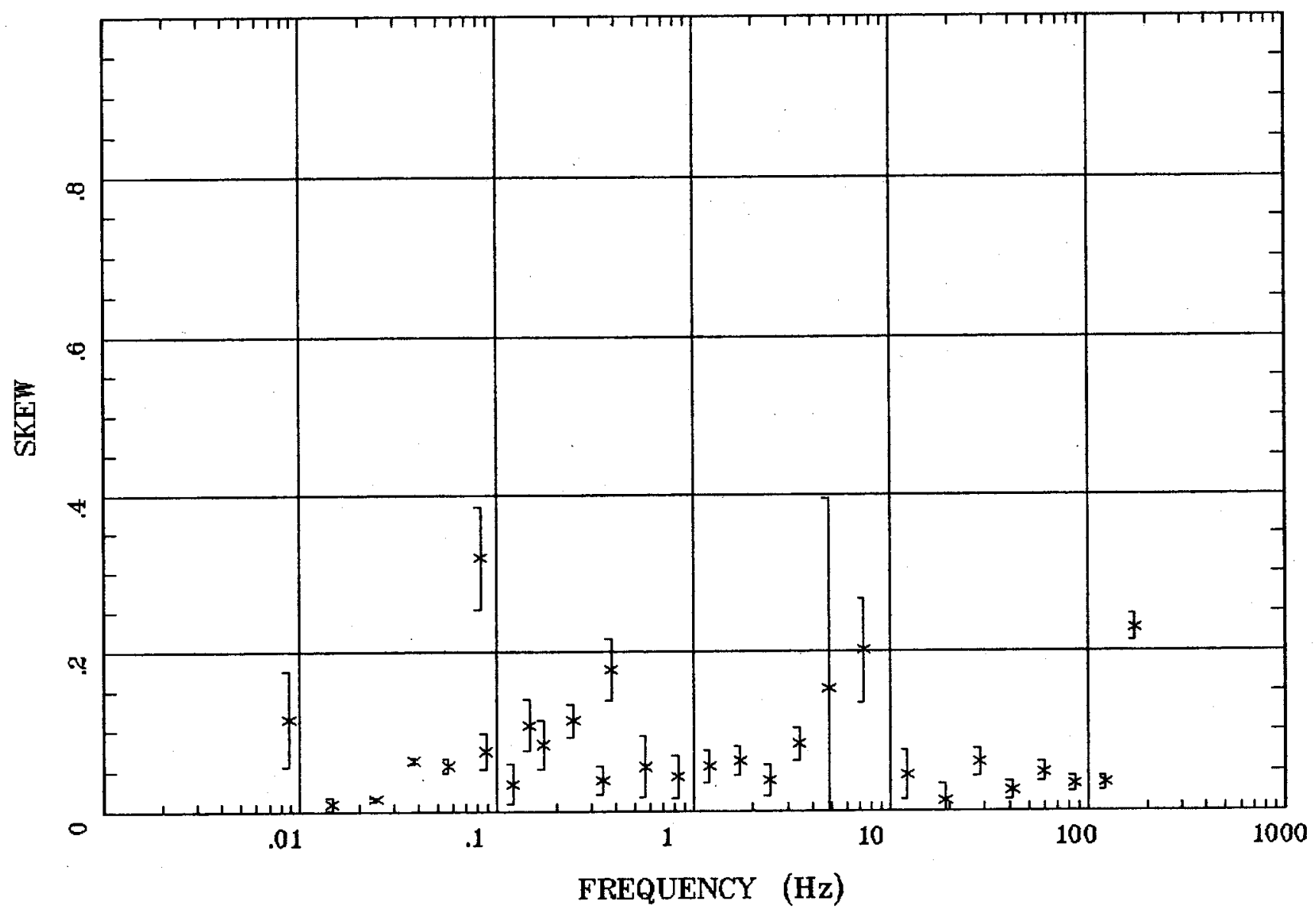

Client: WAter Resources \& DOD

Remote: none

Acquired: $11: 5 \mathrm{Feb}$ 02, 2006

Survey Co:US Geological Survey

Rotation:

Filename: mt60n.avg

Channels: Ch1 Ch2 Ch3 Ch4 Ch5 Ch3 Ch4

Plotted: 12:00 Feb 02, 2006

< EMI - ElectroMagnetic Instruments > 
Station 60

E MULT Coh. Southwest of DAF

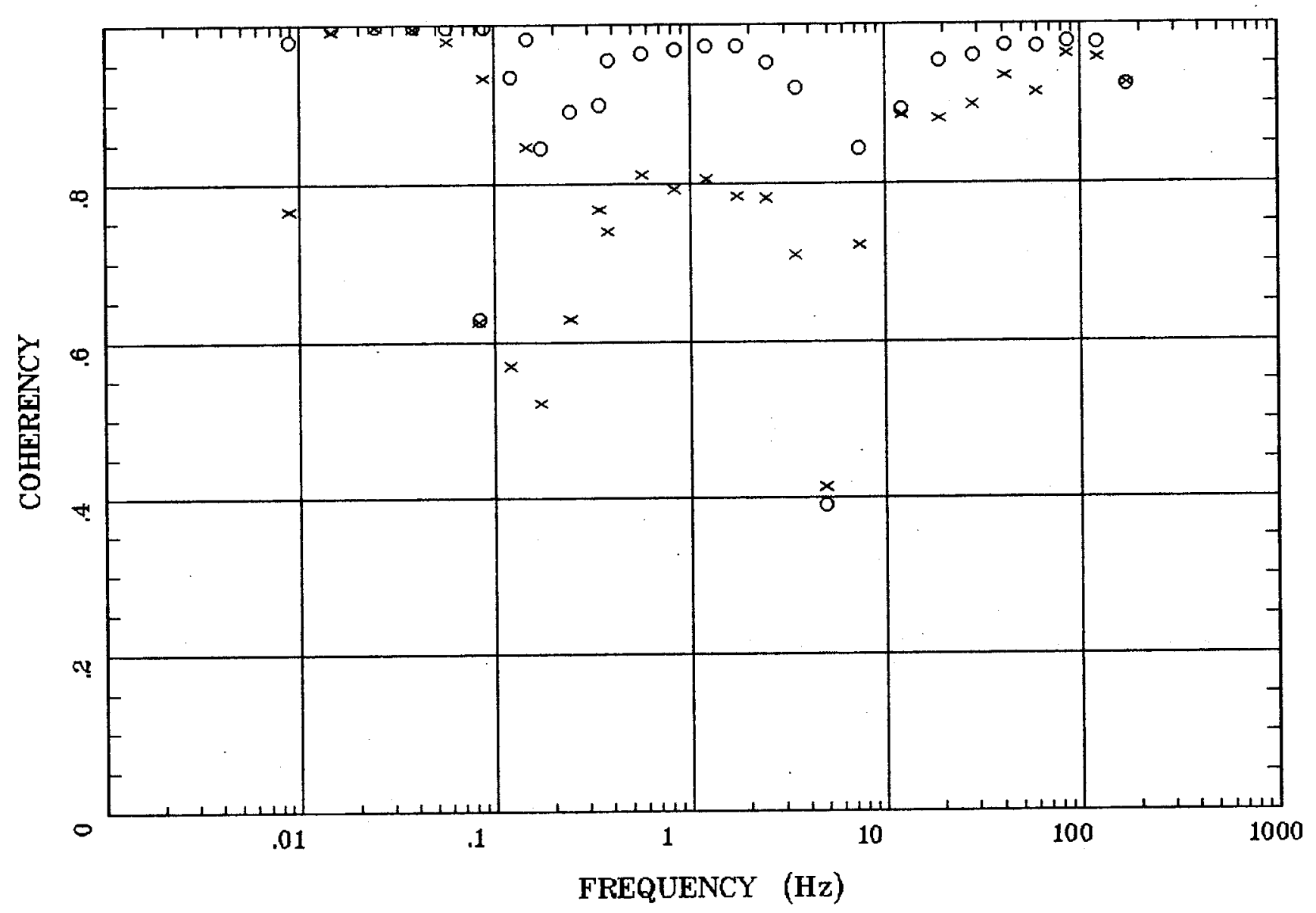

Client: WAter Resources \& DOD

Rotation:

Remote: none

Acquired: 11:5 Feb 02, 2006

Survey Co:US Geological Survey

Filename: mt60n.avg

Channels: Ch1 Ch2 Ch3 Ch4 Ch5 Ch3 Ch4

Plotted: 12:00 Feb 02, 2006

< EMI - ElectroMagnetic Instruments > 
Station 60

\section{POLAR PLOTS}

Southwest of DAF

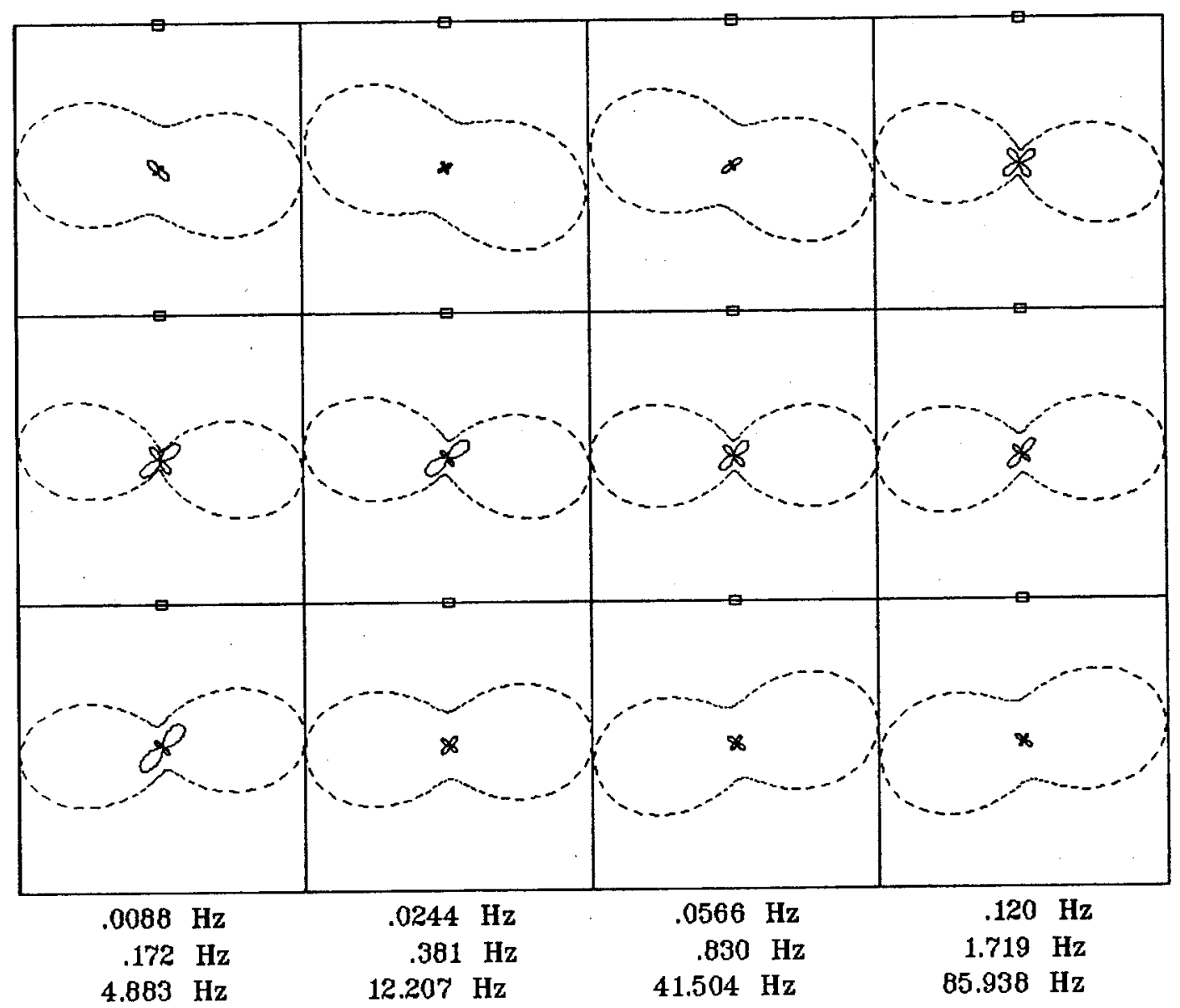

Client: WAter Resources \& DOD Remote: none

Acquired: 11:5 Feb 02, 2006 Survey Co:US Geological Survey

Rotation:

Filename: mt60n.avg

Channels: Ch1 Ch2 Ch3 Ch4 Ch5 Ch3 Ch4

Plotted: 12:00 Feb 02, 2006

< EMI - ElectroMagnetic Instruments > 
Station 60

TIPPER MAGNITUDE

Southwest of DAF

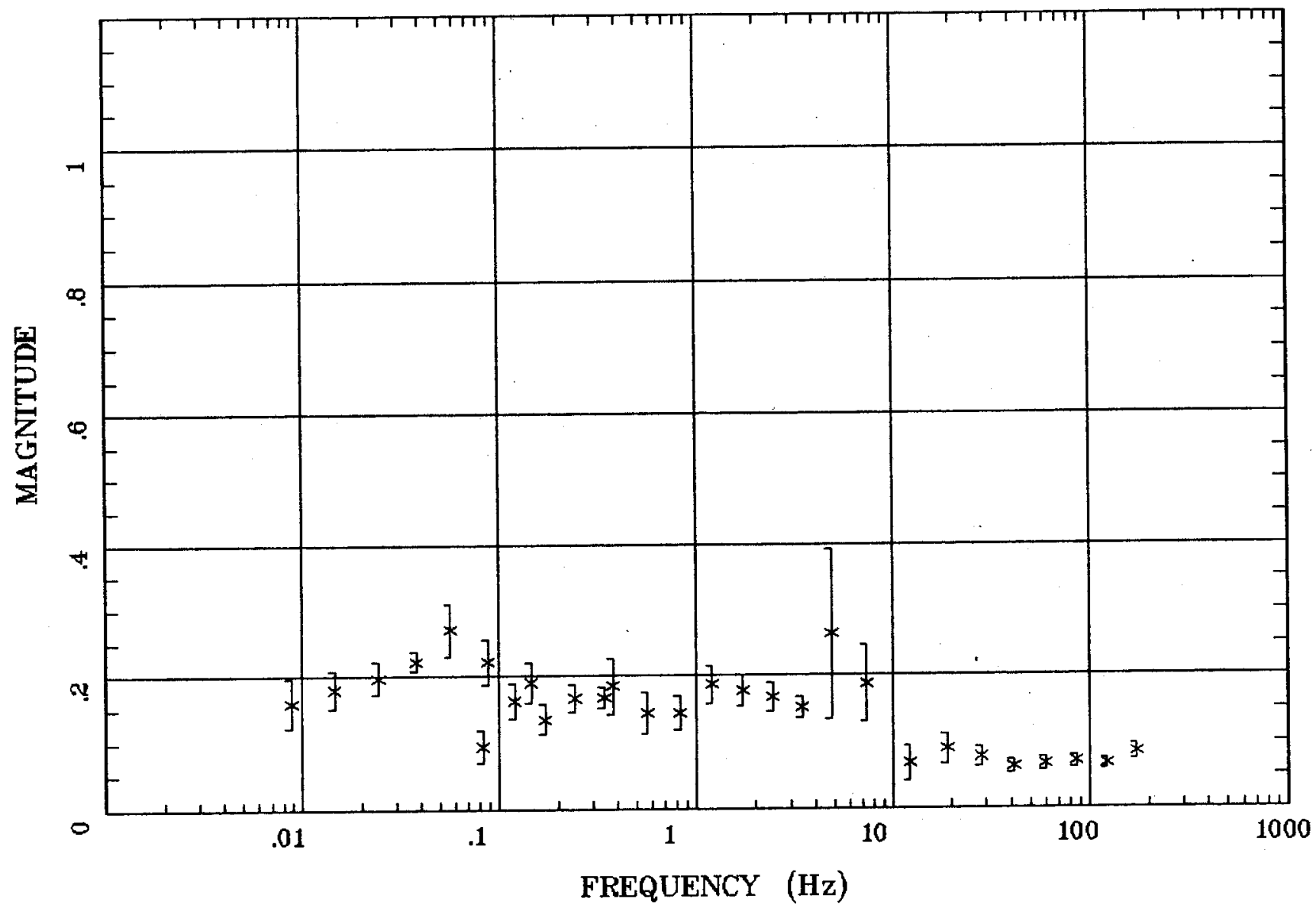

Client: WAter Resources \& DOD

Rotation:

Remote: none

Acquired: 11:5 Feb 02, 2006

Survey Co:US Geological Survey

Filename: mt60n.avg

Channels: Ch1 Ch2 Ch3 Ch4 Ch5 Ch3 Ch4

Plotted: 12:00 Feb 02, 2006

< EMI - ElectroMagnetic Instruments > 
Station 60

TIPPER STRIKE

Southwest of DAF

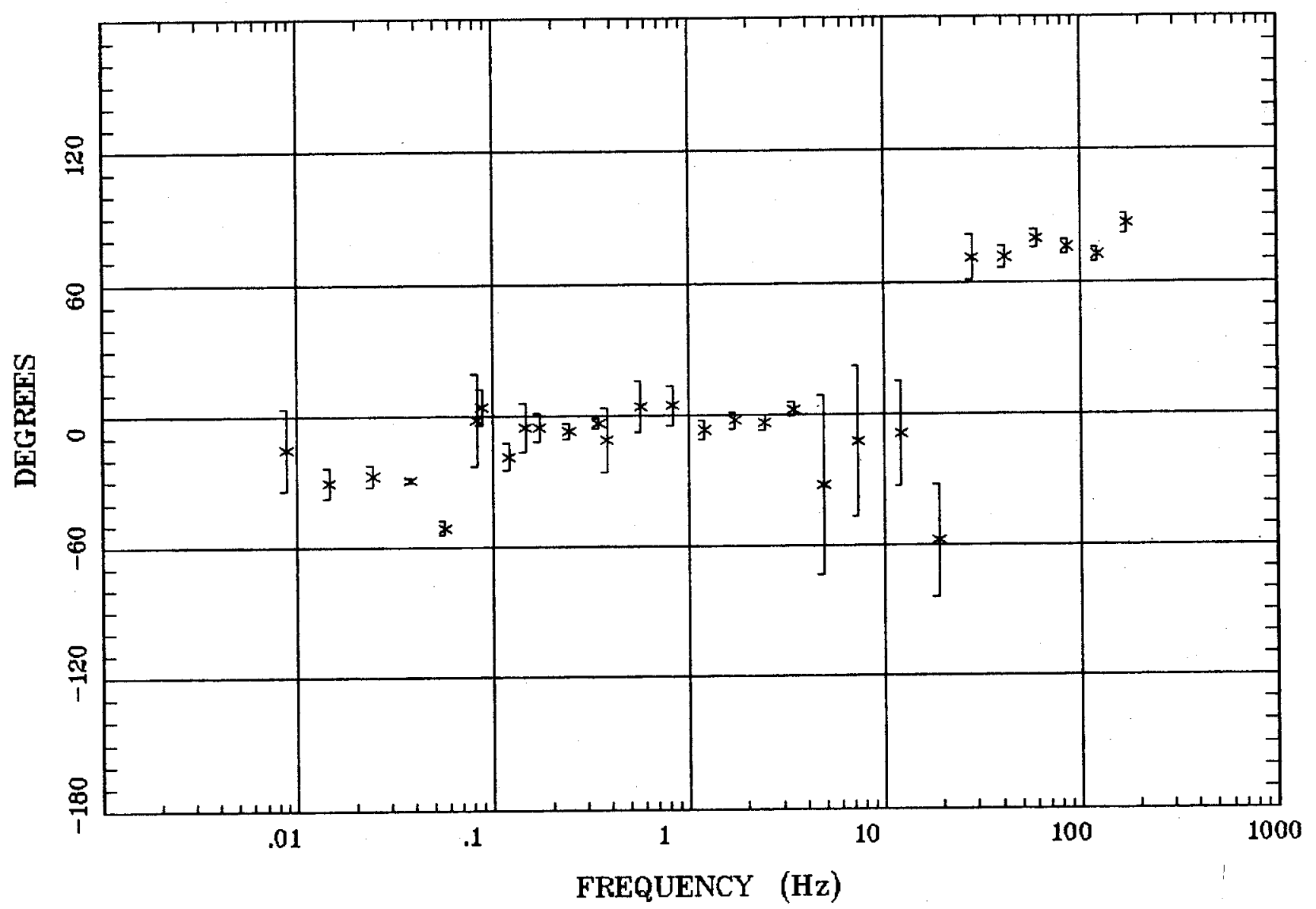

Client: WAter Resources \& DOD Remote: none

Acquired: 11:5 Feb 02, 2006 Survey Co:US Geological Survey
Rotation:

Filename: mt60n.avg

Channels: Ch1 Ch2 Ch3 Ch4 Ch5 Ch3 Ch4

Plotted: 12:00 Feb 02, 2006

< EMI - ElectroMagnetic Instruments > 
Station 60

HzHx.x Coh HzHy.o Southwest of DAF

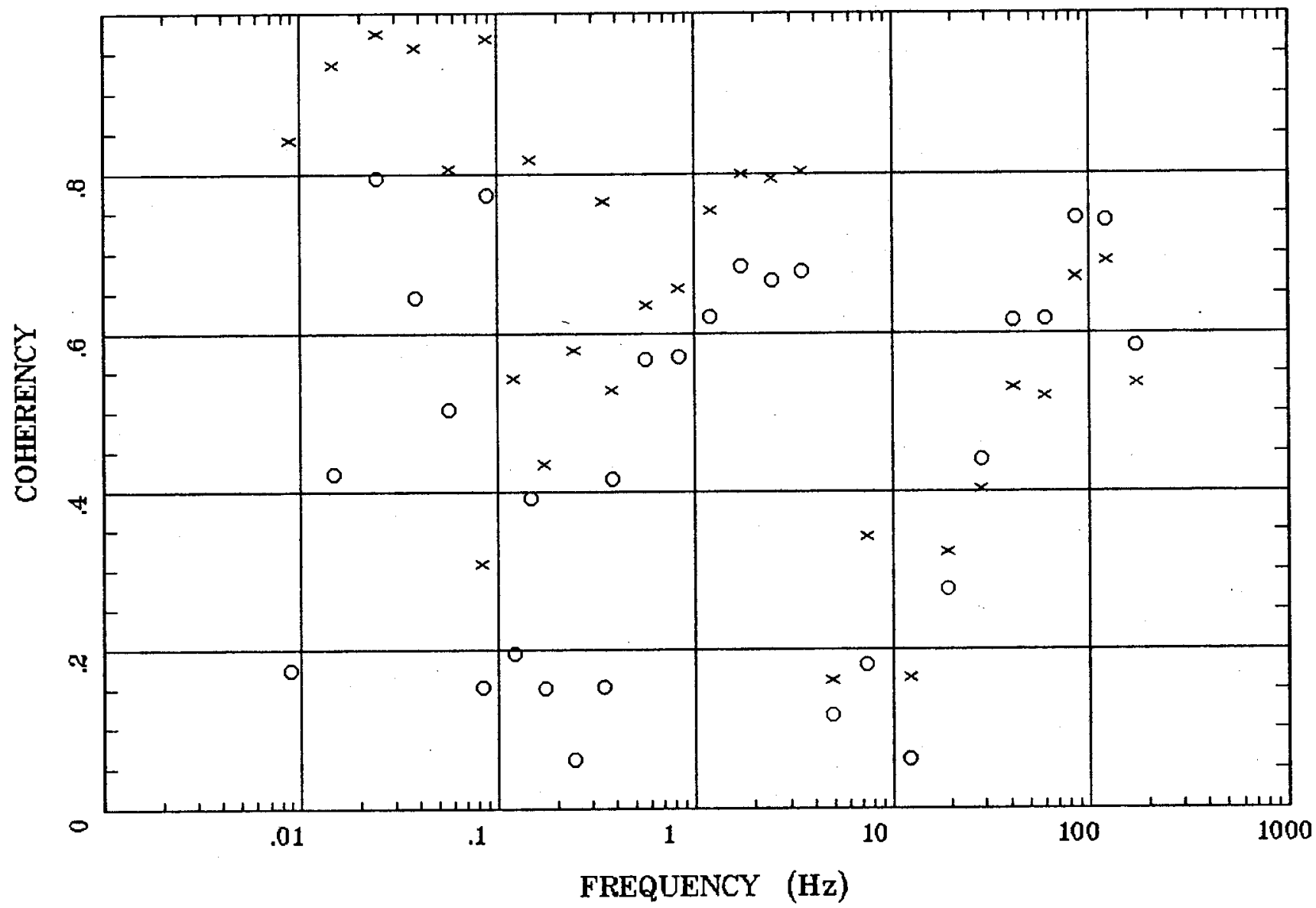

Client: FAter Resources \& DOD Remote: none

Acquired: 11:5 Feb 02, 2006 Survey Co:US Geological Survey
Rotation:

Filename: mt60n.avg

Channels: Ch1 Ch2 Ch3 Ch4 Ch5 Ch3 Ch4

Plotted: 12:00 Feb 02, 2006

< EMI - ElectroMagnetic Instruments > 
Station 61

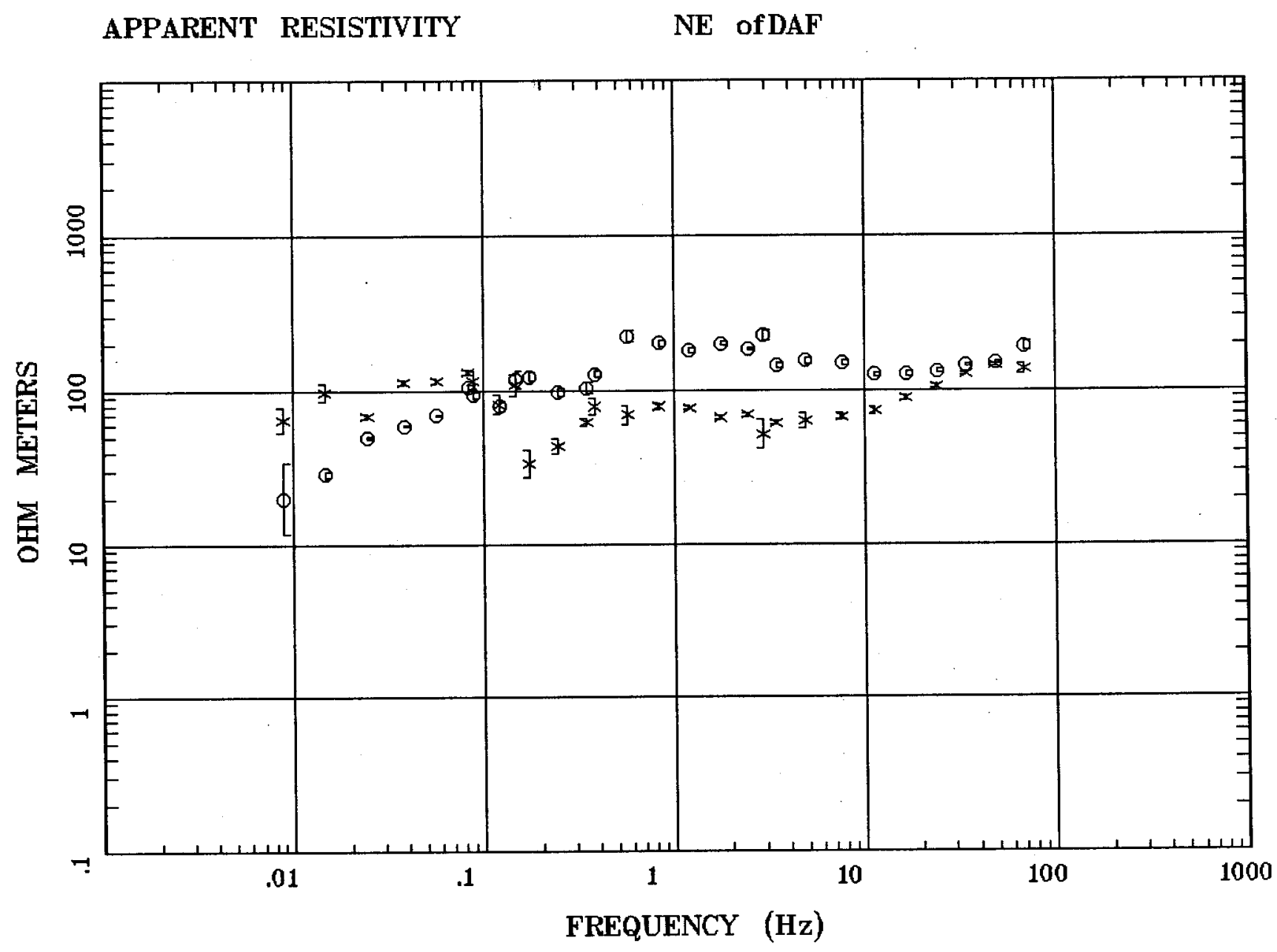

Client: WAter Resources \& DOD Remote: none

Acquired: 04:5 oct 20, 2005 Survey Co:US Geological Survey
Rotation:

Filename: nt61m.avg

Channels: Ch1 Ch2 Ch3 Ch4 Ch5 Ch3 Ch4 Platted: 14:59 Feb 08, 2006

< EMI - ElectroMagnetic Instruments > 


\section{IMPEDANCE PHASE}

NE ofDAF

Station 61

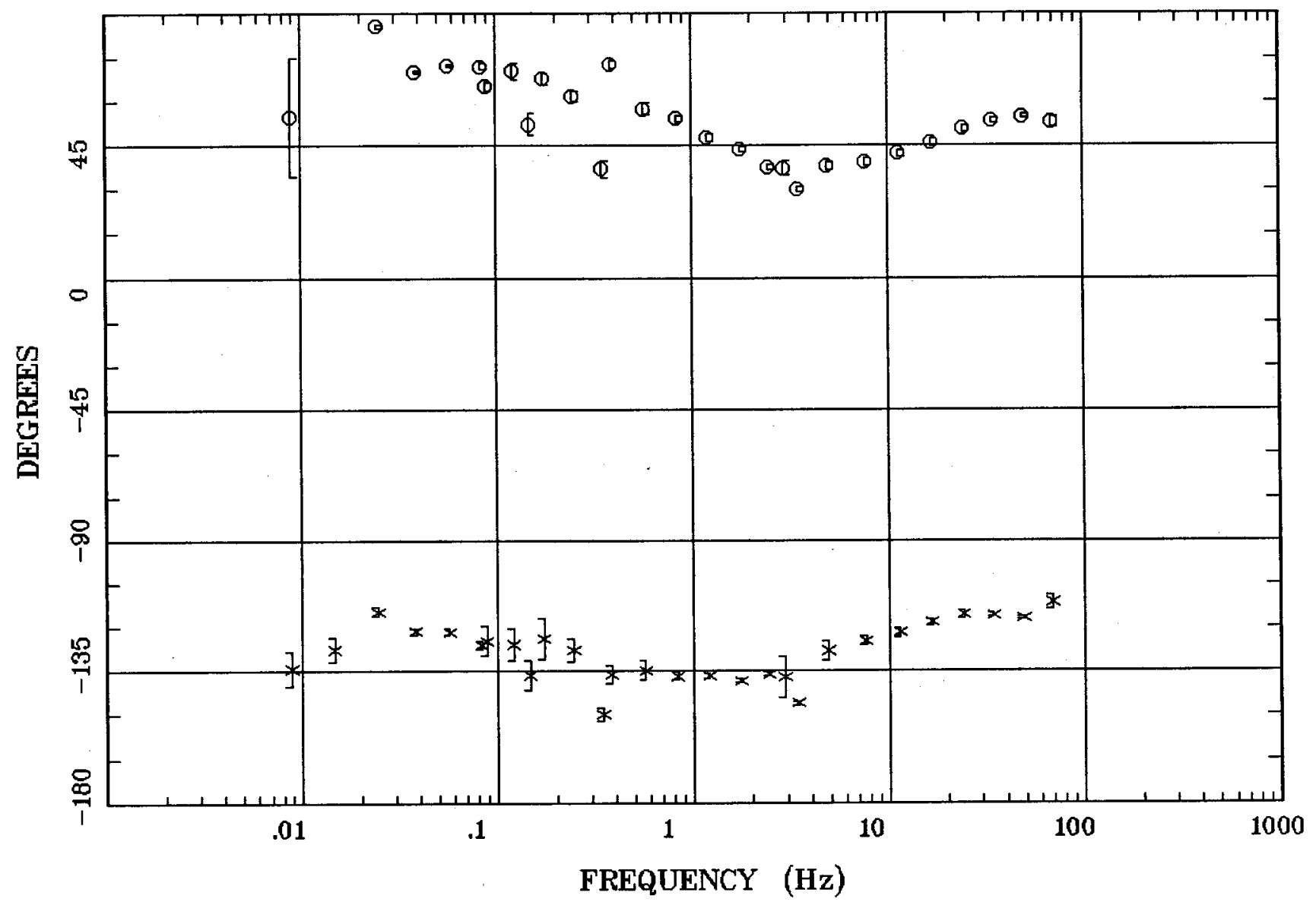

Client: FAter Resources \& DOD Remote: none

Acquired: 04:5 oct 20, 2005 Survey Co:US Geological Survey
Rotation:

Filename: nt61m.avg

Channels: Ch1 Ch2 Ch3 Ch4 Ch5 Ch3 Ch4

Plotted: 14:59 Feb 08, 2006

< EMI - ElectroMagnetic Instruments > 
Station 61

ROTATION ANGLE NE OfDAF

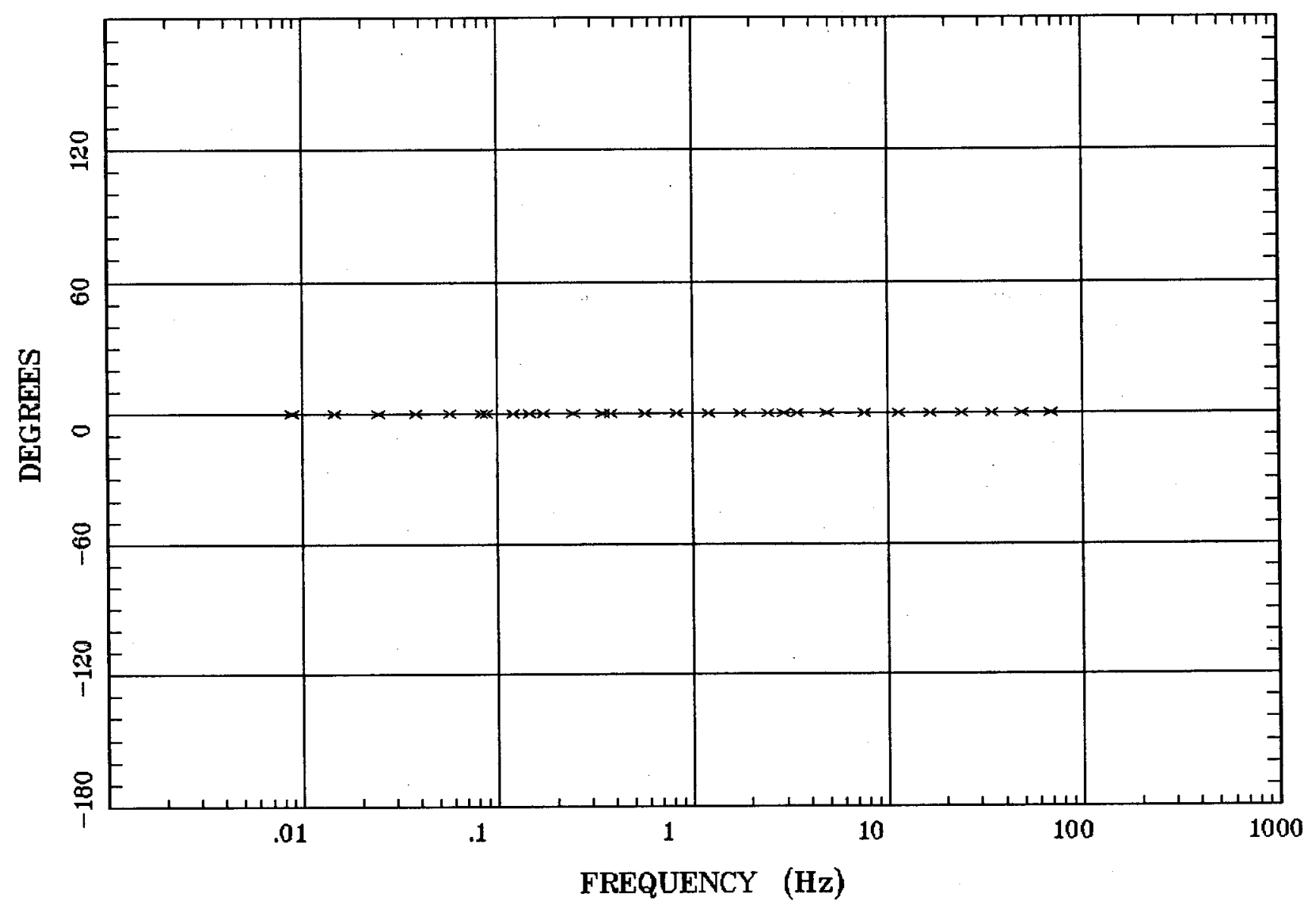

Client: WAter Resources \& DOD

Rotation:

Remote: none

Acquired: 04:5 oct 20, 2005

Survey Co:US Geological Survey

Filename: nt61m.avg

Channels: Ch1 Ch2 Ch3 Ch4 Ch5 Ch3 Ch4

Plotted: 14:59 Feb 08, 2006

< EMI - ElectroMagnetic Instruments > 


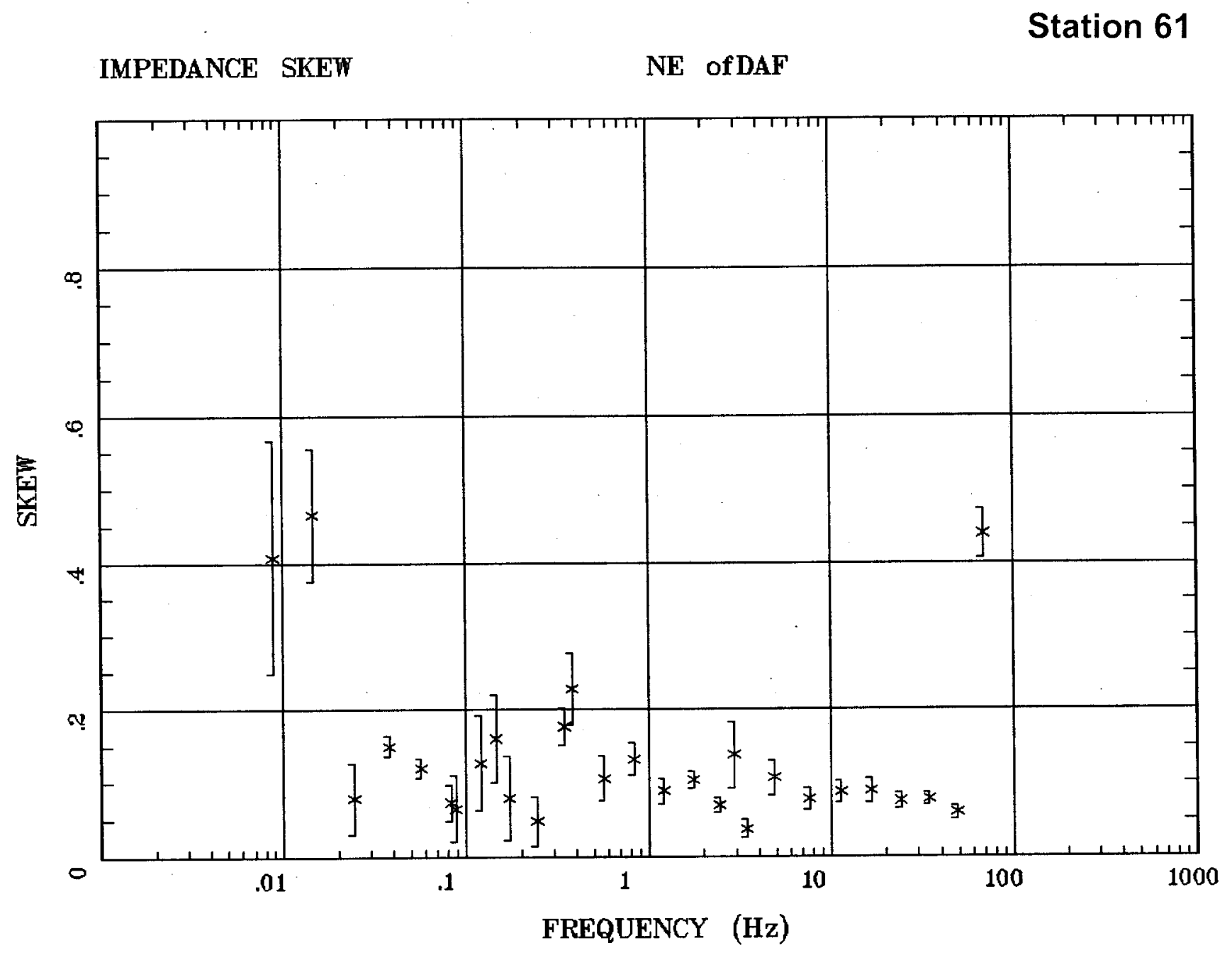

Client: WAter Resources \& DOD Remote: none Acquired: 04:5 oct 20, 2005 Survey Co:US Geological Survey

Rotation:

Filename: nt61m.avg

Channels: Ch1 Ch2 Ch3 Ch4 Ch5 Ch3 Ch4

Plotted: 14:59 Feb 08, 2006

< EMI - ElectroMagnetic Instruments > 


\section{Station 61}

E MULT Coh.

NE OfDAF

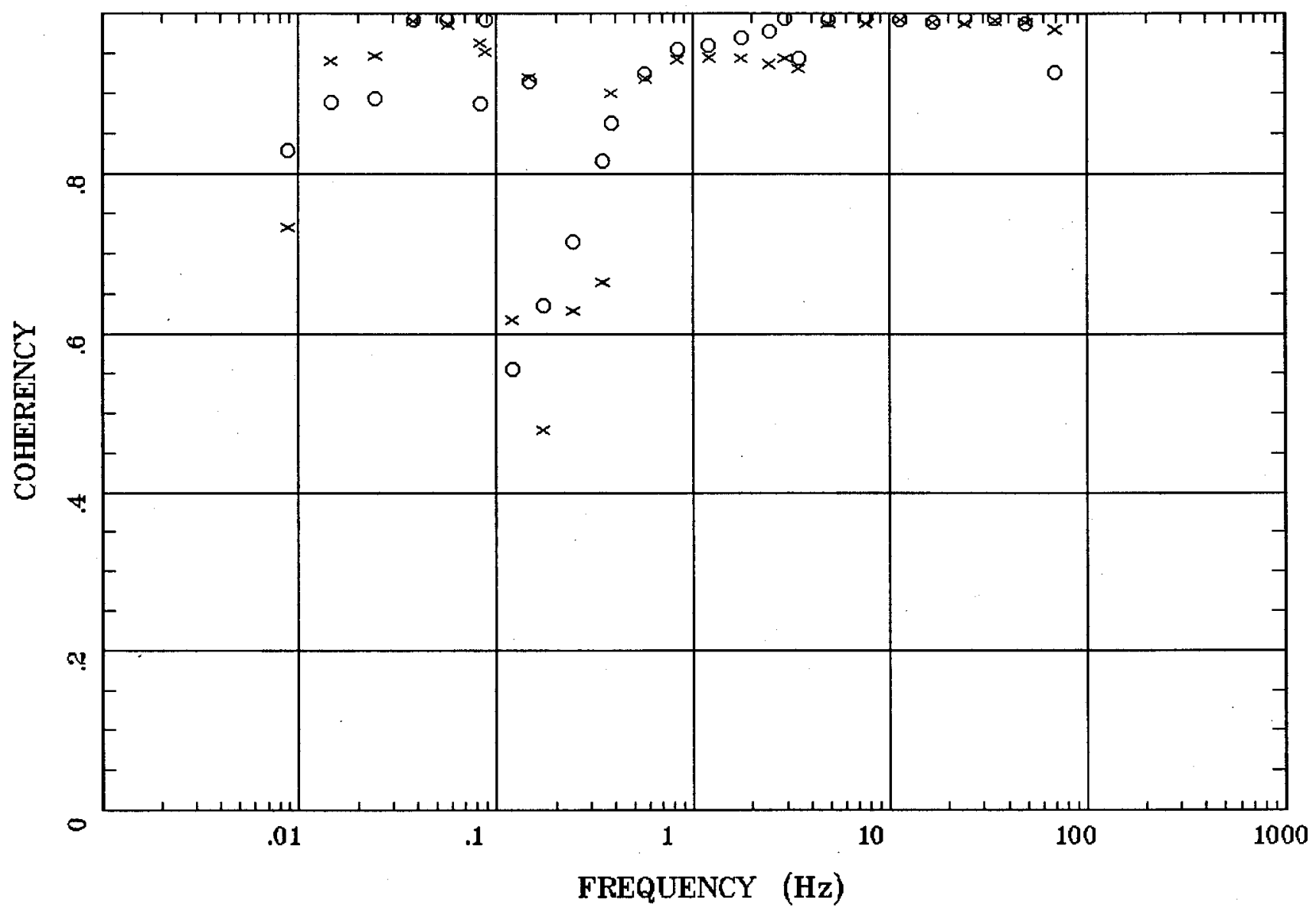

Client: WAter Resources \& DOD

Rotation:

Remote: none

Filename: nt61m.avg

Channels: Ch1 Ch2 Ch3 Ch4 Ch5 Ch3 Ch4

Acquired: 04:5 oct 20, 2005

Survey Co:US Geological Survey

Plotted: 14:59 Feb 08, 2006

< EMI - ElectroMagnetic Instruments > 
Station 61

POLAR PLOTS

NE ofDAF

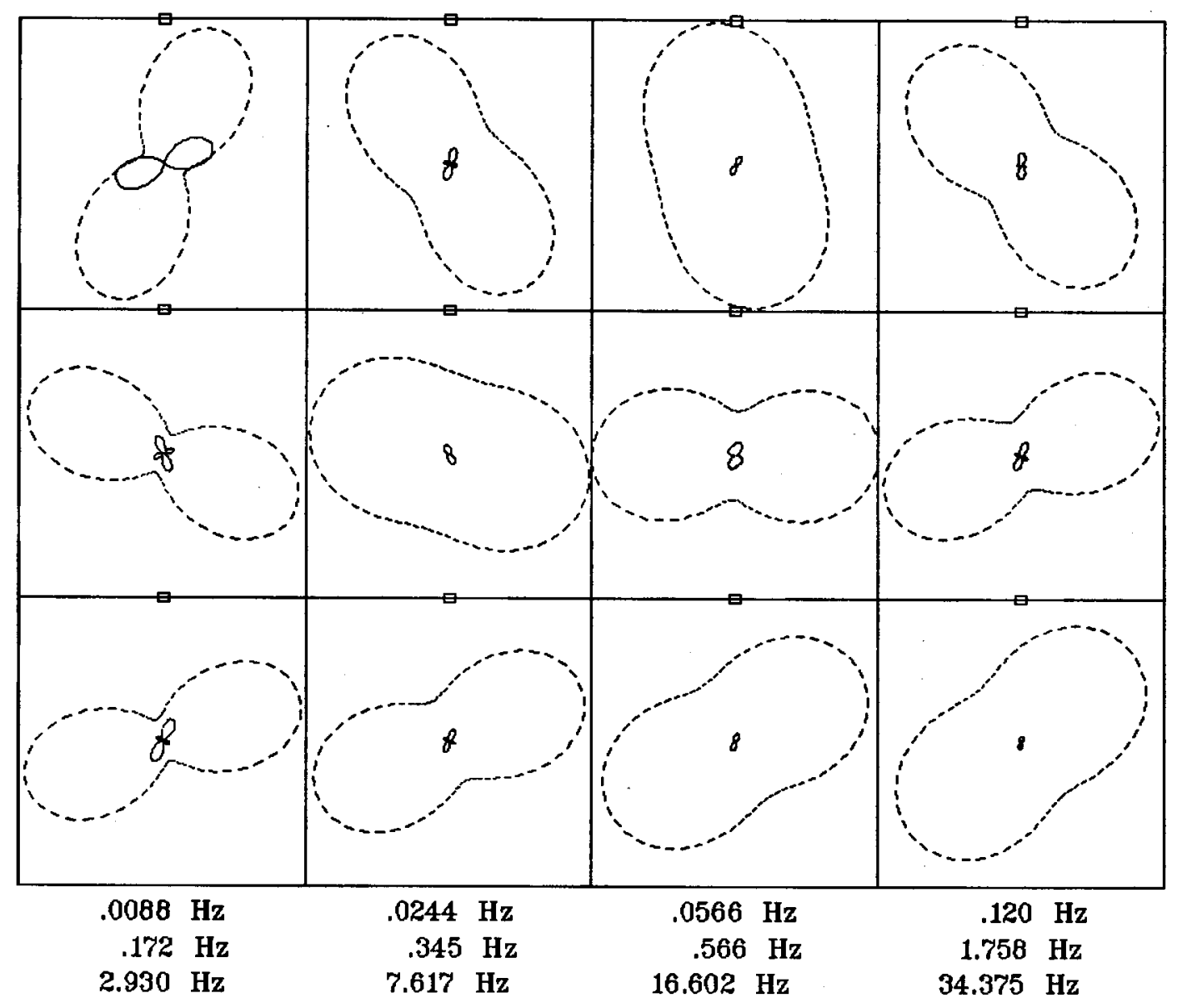

Client: FAter Resources \& DOD Remote: none

Acquired: 04:5 Oct 20, 2005

Survey Co:US Geological Survey

Rotation:

Filename: nt61m.avg

Channels: Ch1 Ch2 Ch3 Ch4 Ch5 Ch3 Ch4

Plotted: 14:59 Feb 08, 2006

< EMI - ElectroMagnetic Instruments > 
Station 61

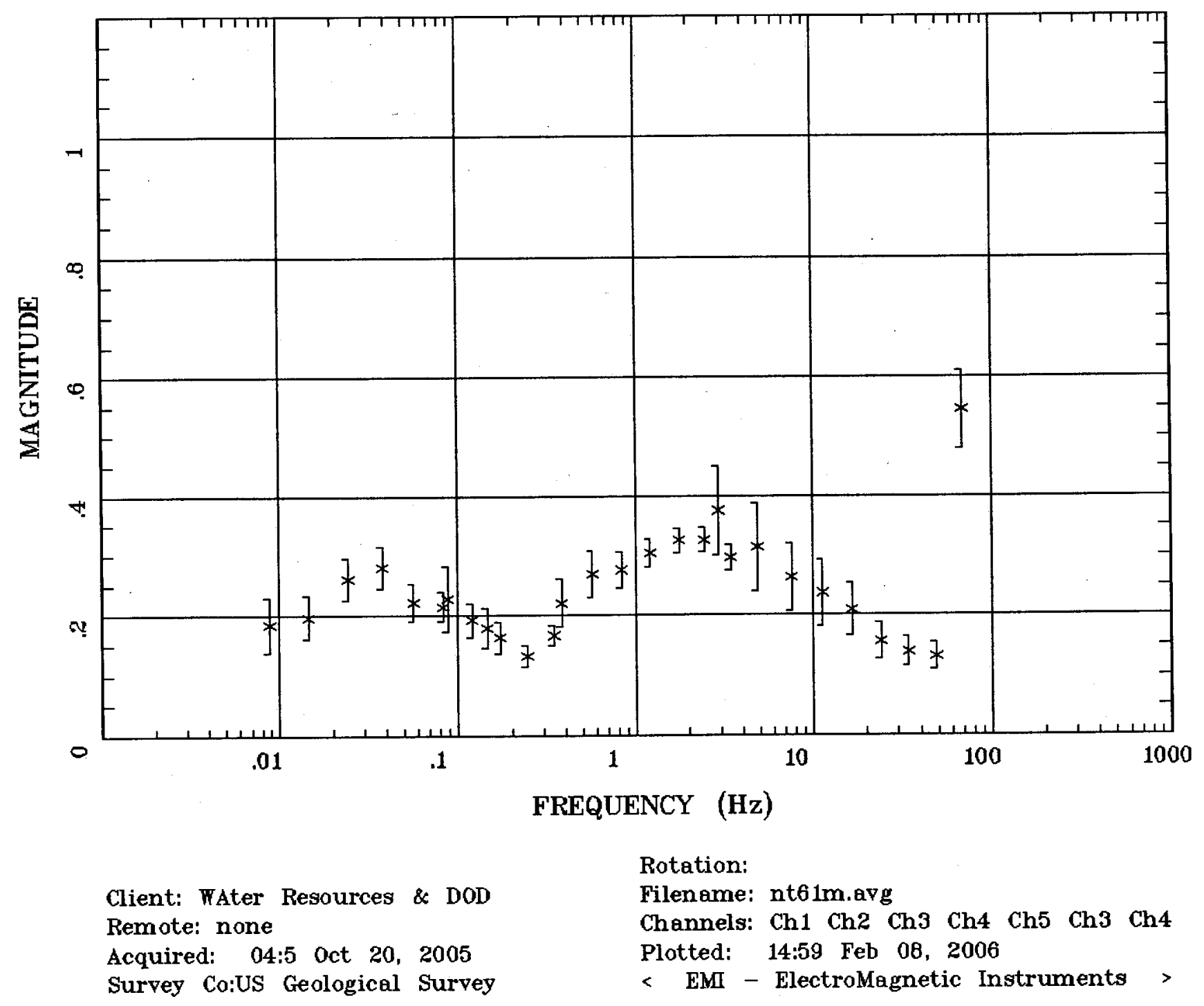




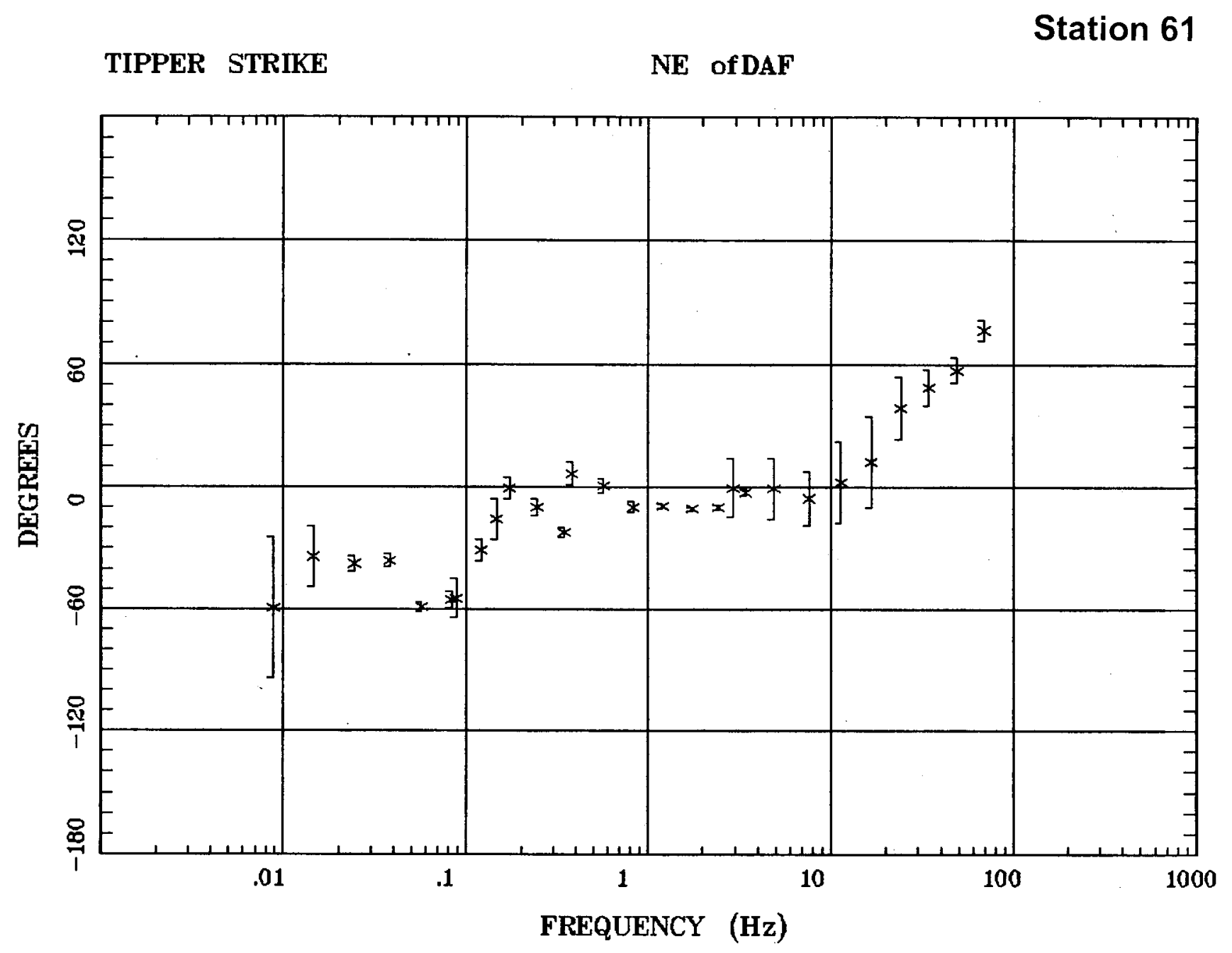

Client: WAter Resources \& DOD Remote: none Acquired: $04: 5$ oct 20, 2005 Survey Co:US Geological Survey

Rotation:

Filename: nt61m.avg Channels: Ch1 Ch2 Ch3 Ch4 Ch5 Ch3 Ch4 Plotted: 14:59 Feb 08, 2006

< EMI - ElectroMagnetic Instruments > 
Station 61

HzHx.x Coh HzHy.o NE of DAF

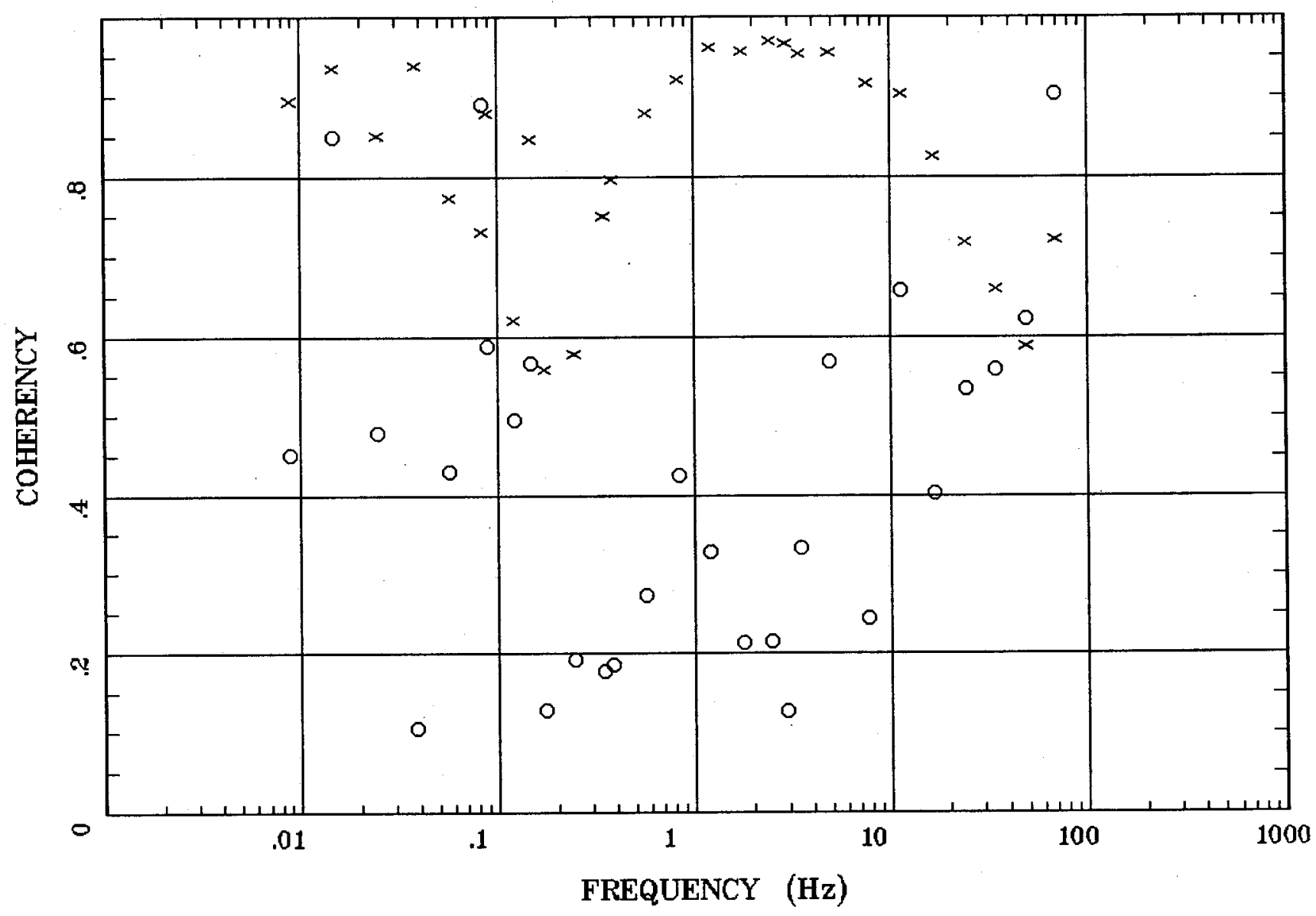

Client: WAter Resources \& DOD Remate: none Acquired: 04:5 oct 20, 2005 Survey Co:US Geological Survey
Rotation:

Filename: nt61m.avg

Channels: Ch1 Ch2 Ch3 Ch4 Ch5 Ch3 Ch4 Plotted: 14:59 Feb 08, 2006

< EMI - ElectroMagnetic Instruments > 
Station 62

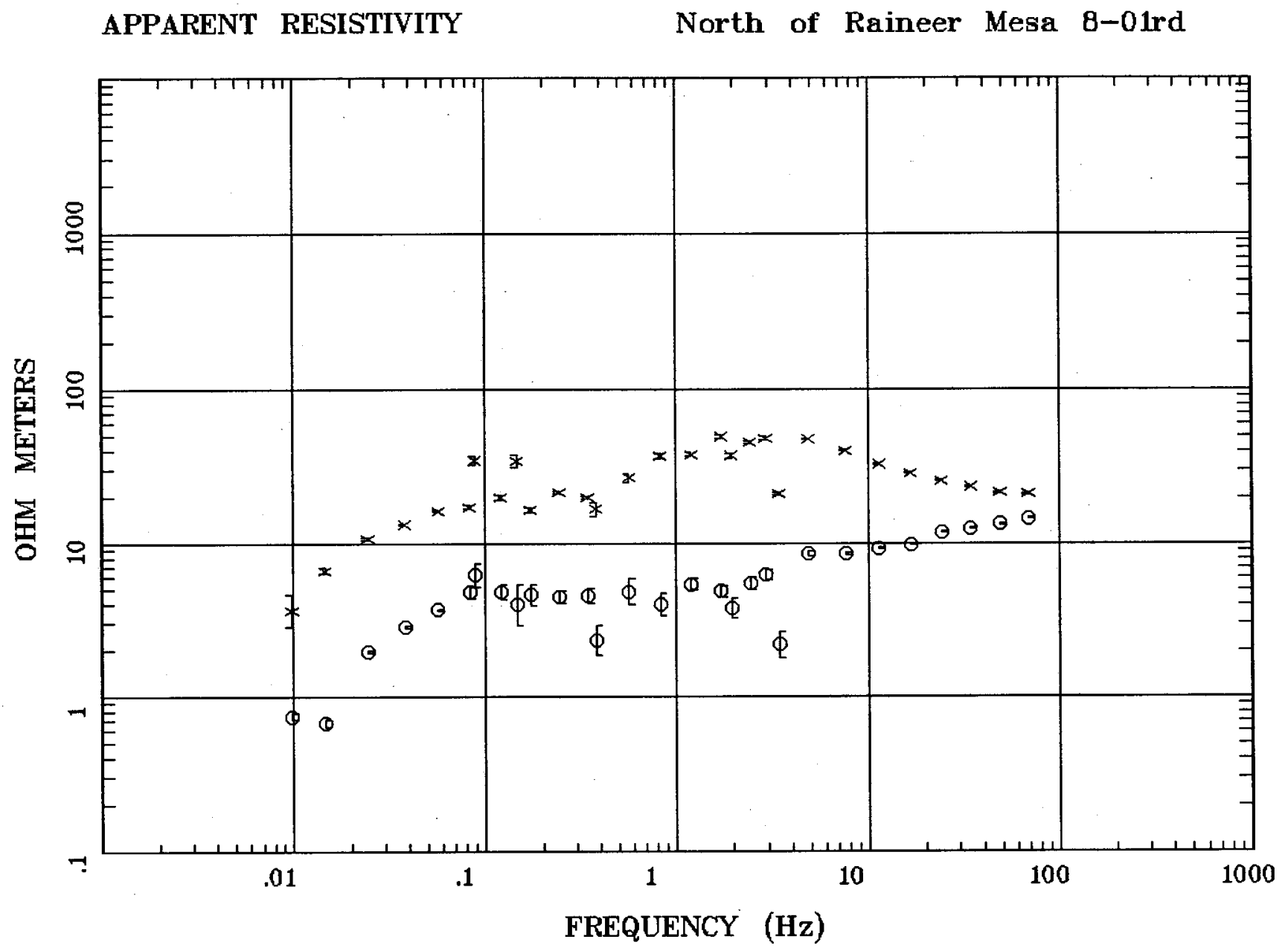

Client: WAter Resources \& DOD Remote: none

Acquired: 13:5 Feb 01, 2006 Survey Co:US Geological Survey
Rotation:

Filename: nt62n.avg Channels: Ch1 Ch2 Ch3 Ch4 Ch5 Ch3 Ch4 Plotted: 15:18 Feb 08, 2006

< EMI - ElectroMagnetic Instruments > 


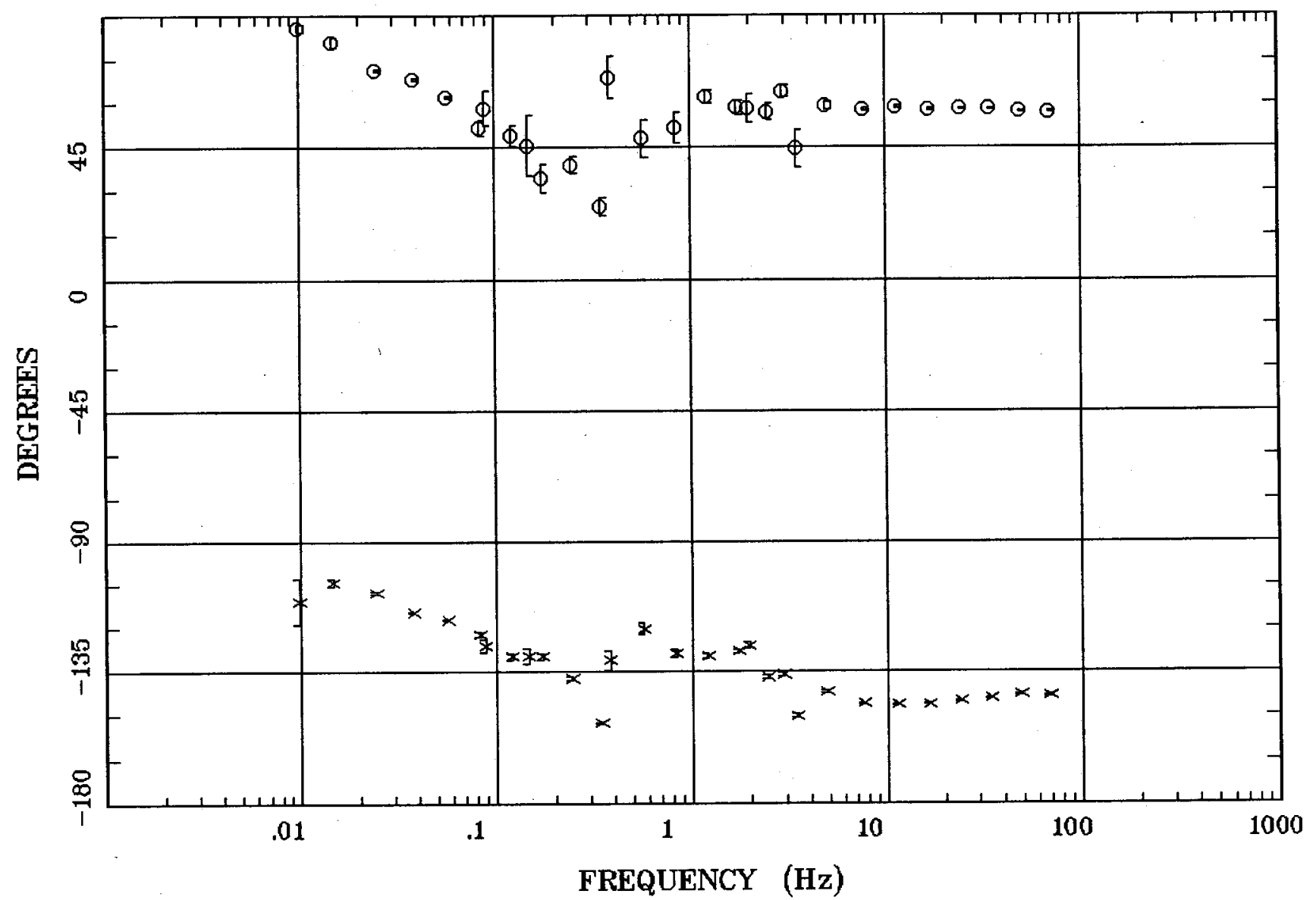

Client: WAter Resources \& DOD Remote: none Acquired: 13:5 Feb 01, 2006 Survey Co:US Geological Survey
North of Raineer Mesa 8-01rd

Rotation:

Filename: nt62n.evg

Channels: Ch1 Ch2 Ch3 Ch4 Ch5 Ch3 Ch4

Plotted: 15:18 Feb 08, 2006

< EMI - ElectroMagnetic Instruments > 
Station 62

ROTATION ANGLE North of Raineer Mesa 8-01rd

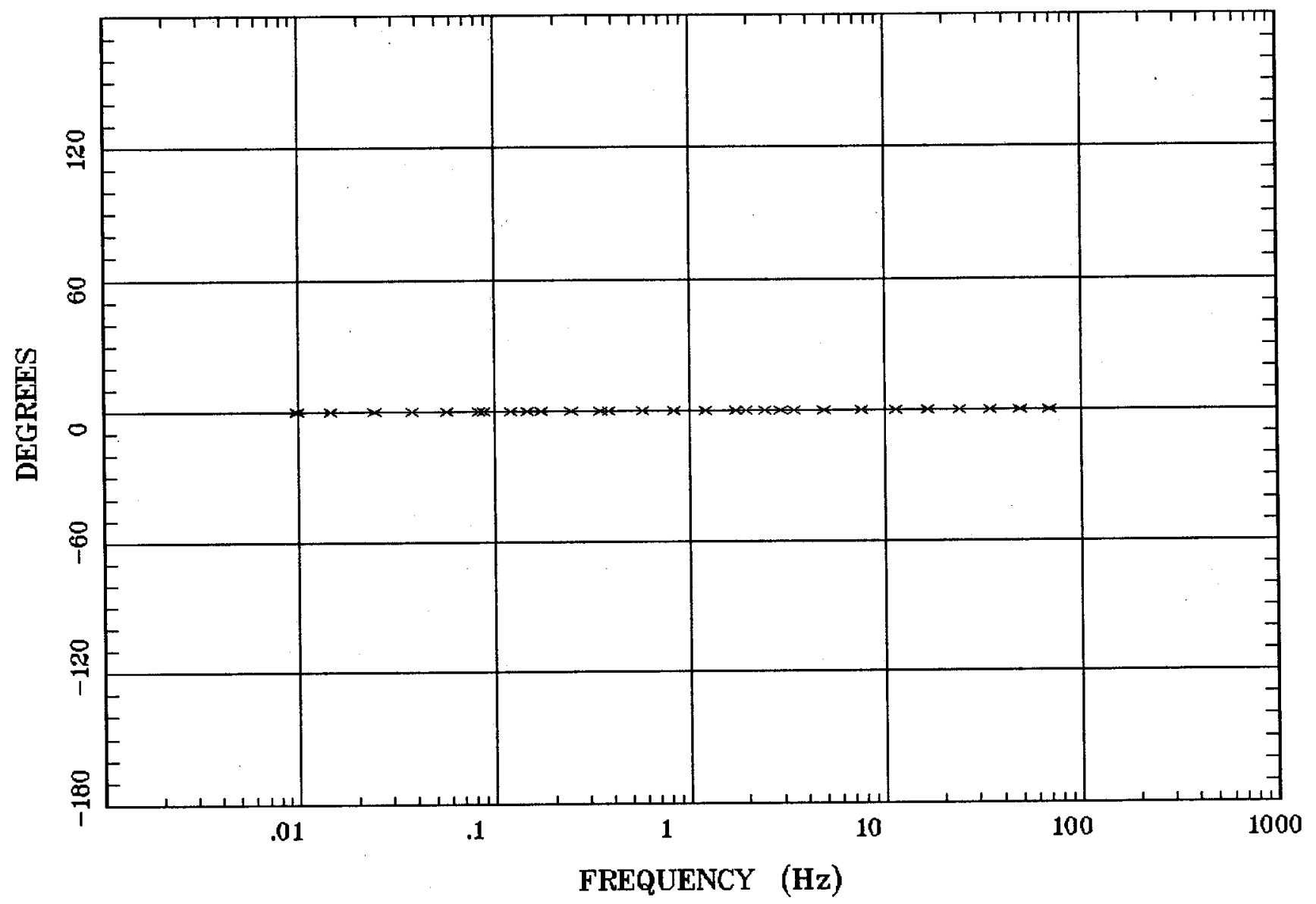

Client: WAter Resources \& DOD Remote: none Acquired: 13:5 Feb 01, 2006 Survey Co:US Geological Survey
Rotation:

Filename: nt62n.avg Channels: Ch1 Ch2 Ch3 Ch4 Ch5 Ch3 Ch4 Plotted: 15:18 Feb 08, 2006

< EMI - ElectroMagnetic Instruments > 
Station 62

IMPEDANCE SKEW

North of Raineer Mesa 8-01rd

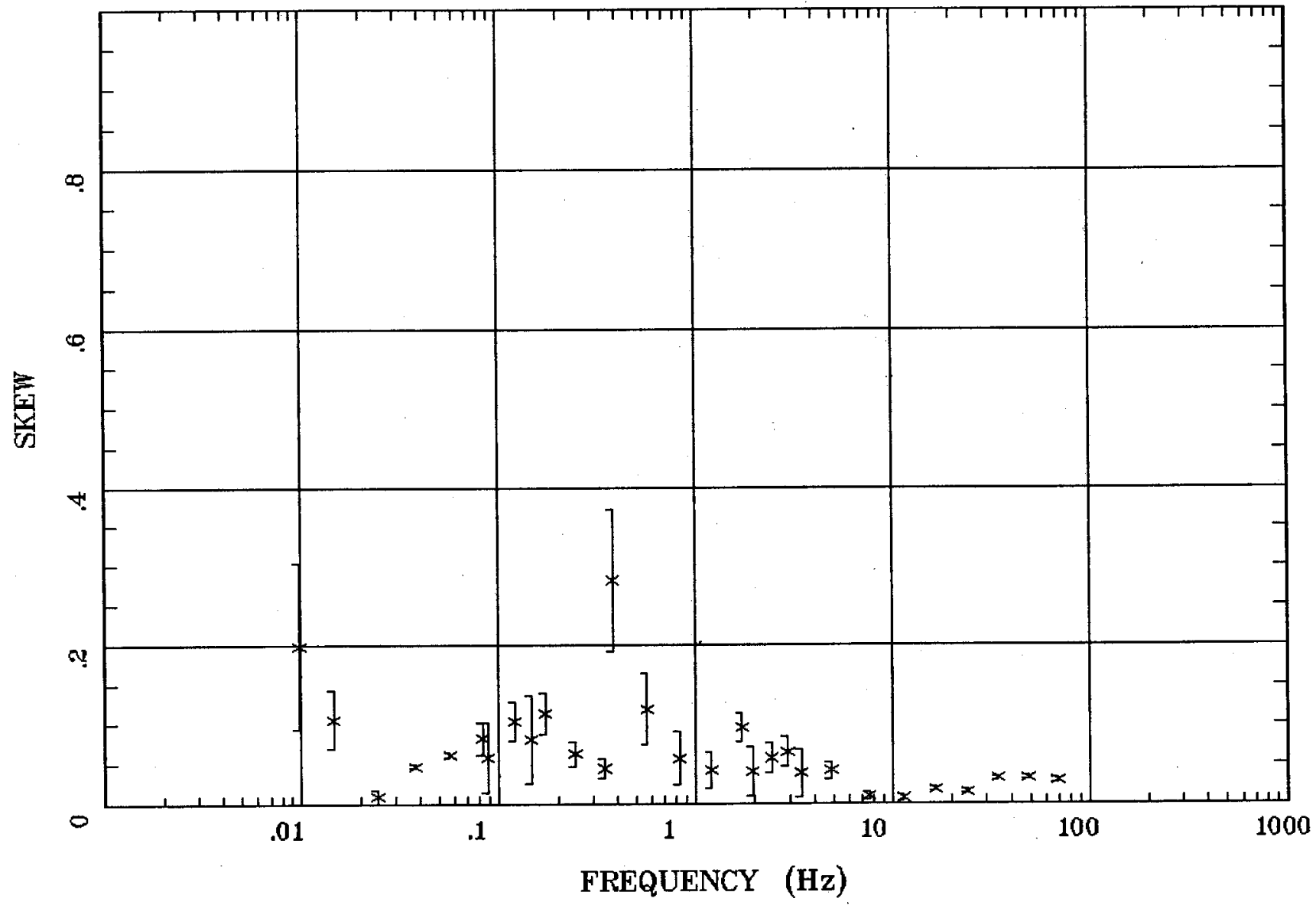

Client: WAter Resources \& DOD Remote: none

Acquired: 13:5 Feb 01, 2006 Survey Co:US Geological Survey

Rotation:

Filename: nt62n.avg

Channels: Ch1 Ch2 Ch3 Ch4 Ch5 Ch3 Ch4

Plotted: 15:18 Feb 08, 2006

< EMI - ElectroMagnetic Instruments > 
Station 62

E MULT Coh.

North of Raineer Mesa 8-01rd

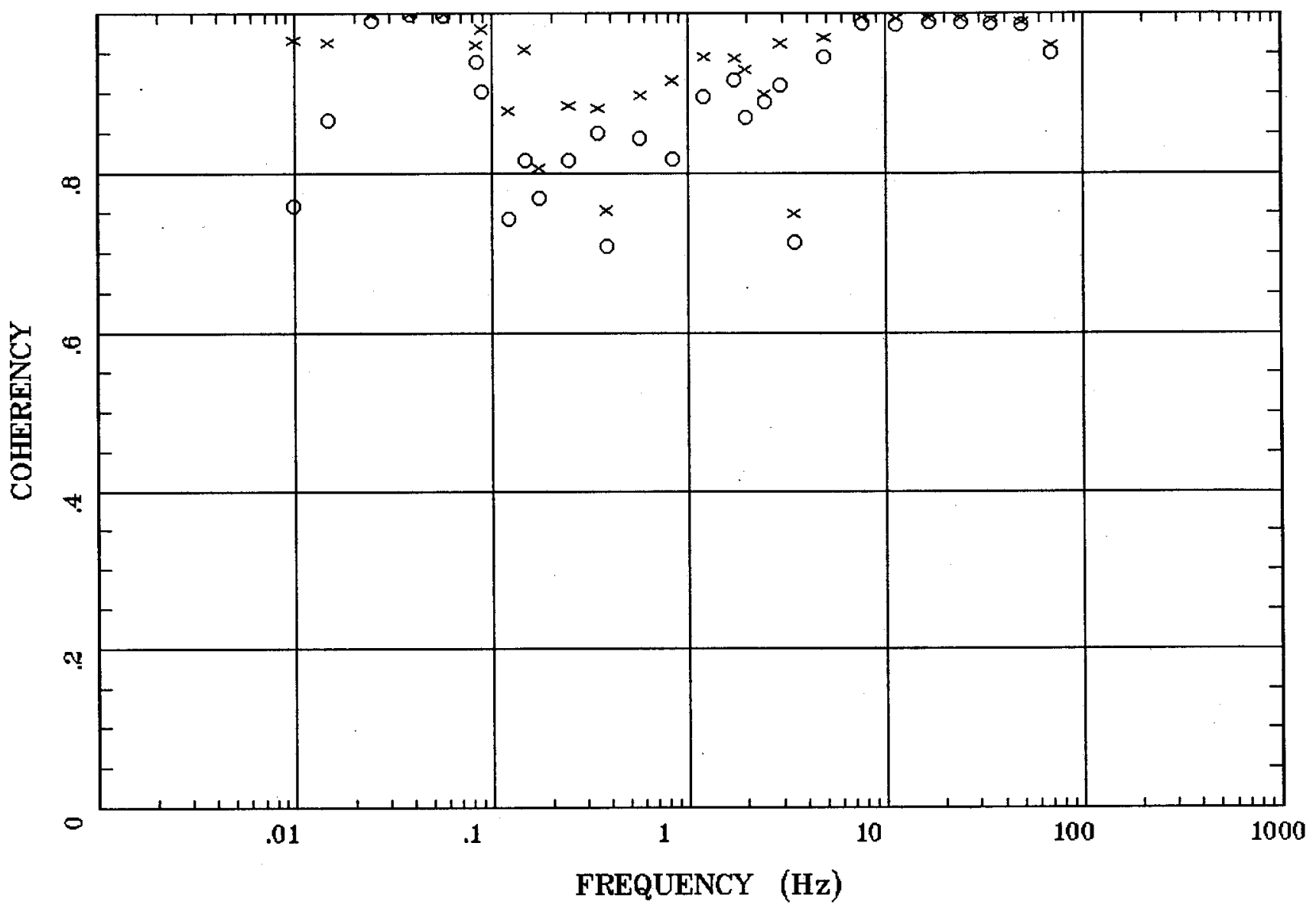

Client: WAter Resources \& DOD Remote: none

Acquired: 13:5 Feb 01, 2006 Survey Co:US Geological Survey
Rotation:

Filename: nt62n.avg Channels: Ch1 Ch2 Ch3 Ch4 Ch5 Ch3 Ch4 Plotted: 15:18 Feb 08, 2006

< EMI - ElectroMagnetic Instruments > 
Station 62

POLAR PLOTS North of Raineer Mesa 8-01rd

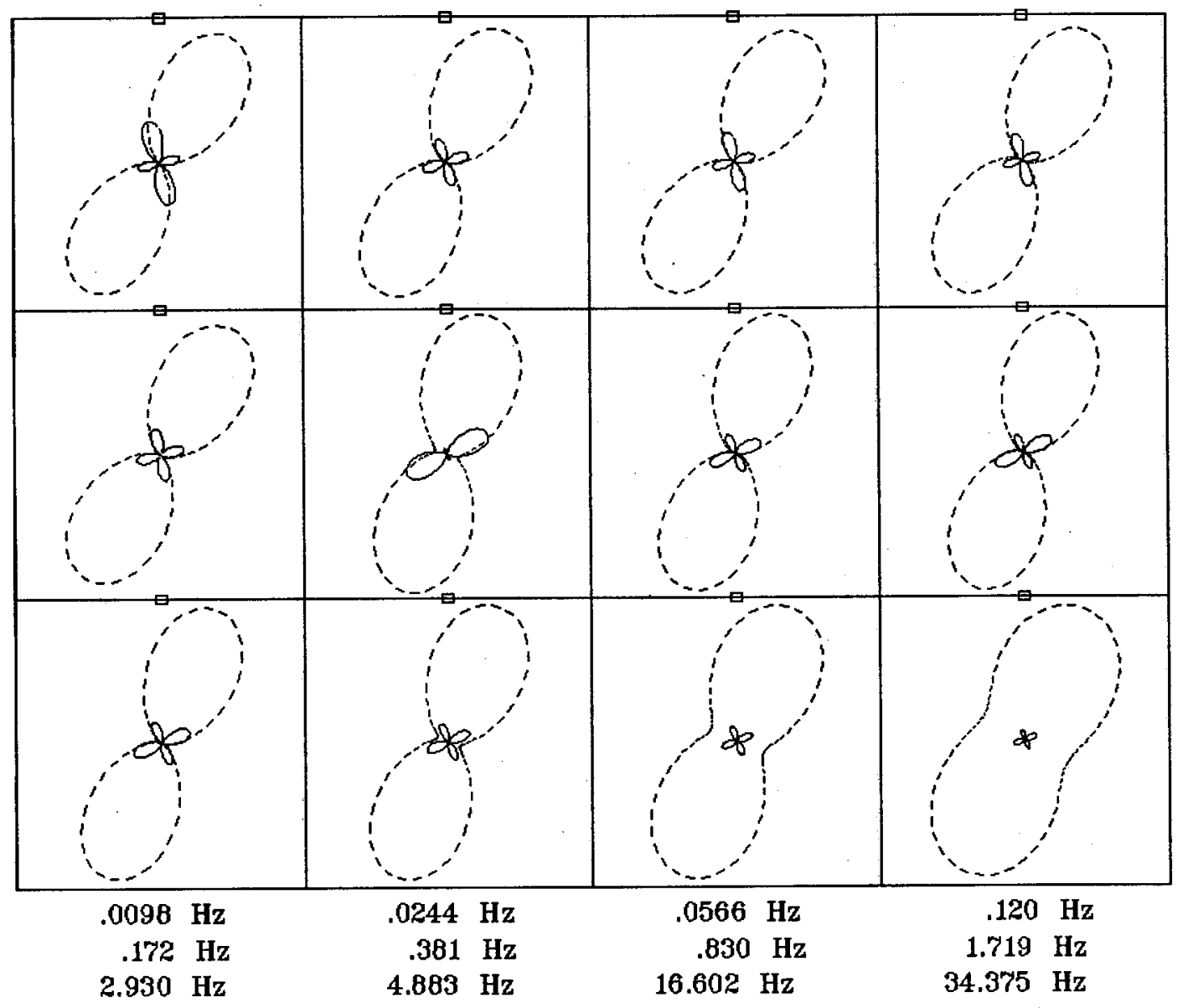

Client: WAter Resources \& DOD

Rotation:

Remote: none

Filename: nt62n.avg

Channels: Ch1 Ch2 Ch3 Ch4 Ch5 Ch3 Ch4

Acquired: 13:5 Feb 01, 2006

Plotted: $15: 18$ Feb 08, 2006

Survey Co:US Geological Survey

< EMI - ElectroMagnetic Instruments > 
Station 62

TIPPER MAGNITUDE

North of Raineer Mesa 8-01rd

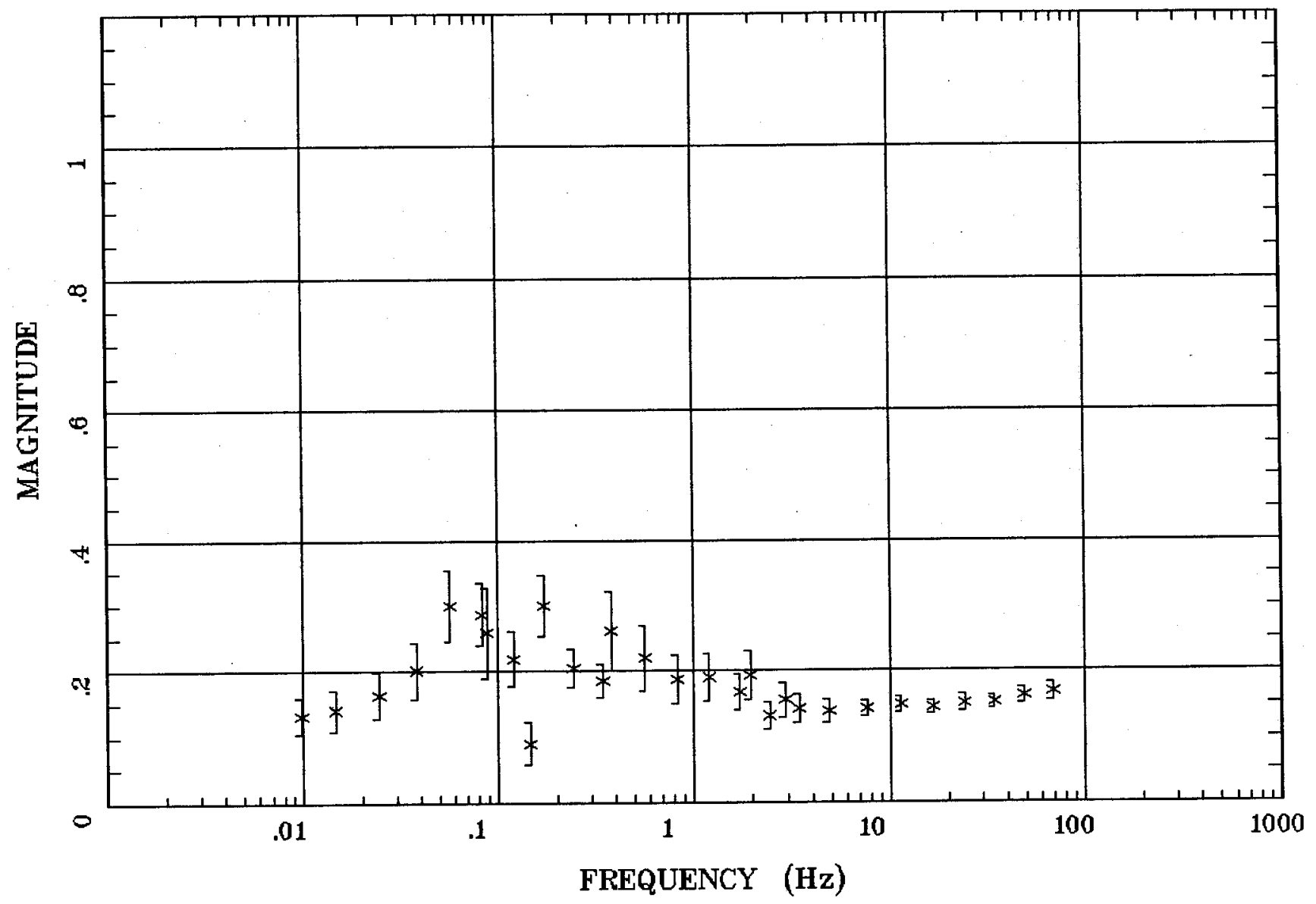

Client: WAter Resources \& DOD

Rotation:

Remote: none

Acquired: 13:5 Feb 01, 2006

Survey Co:US Geological Survey

Filename: nt62n.avg

Channels: Ch1 Ch2 Ch3 Ch4 Ch5 Ch3 Ch4

Plotted: 15:19 Feb 08, 2006

< EMI - ElectroMagnetic Instruments > 


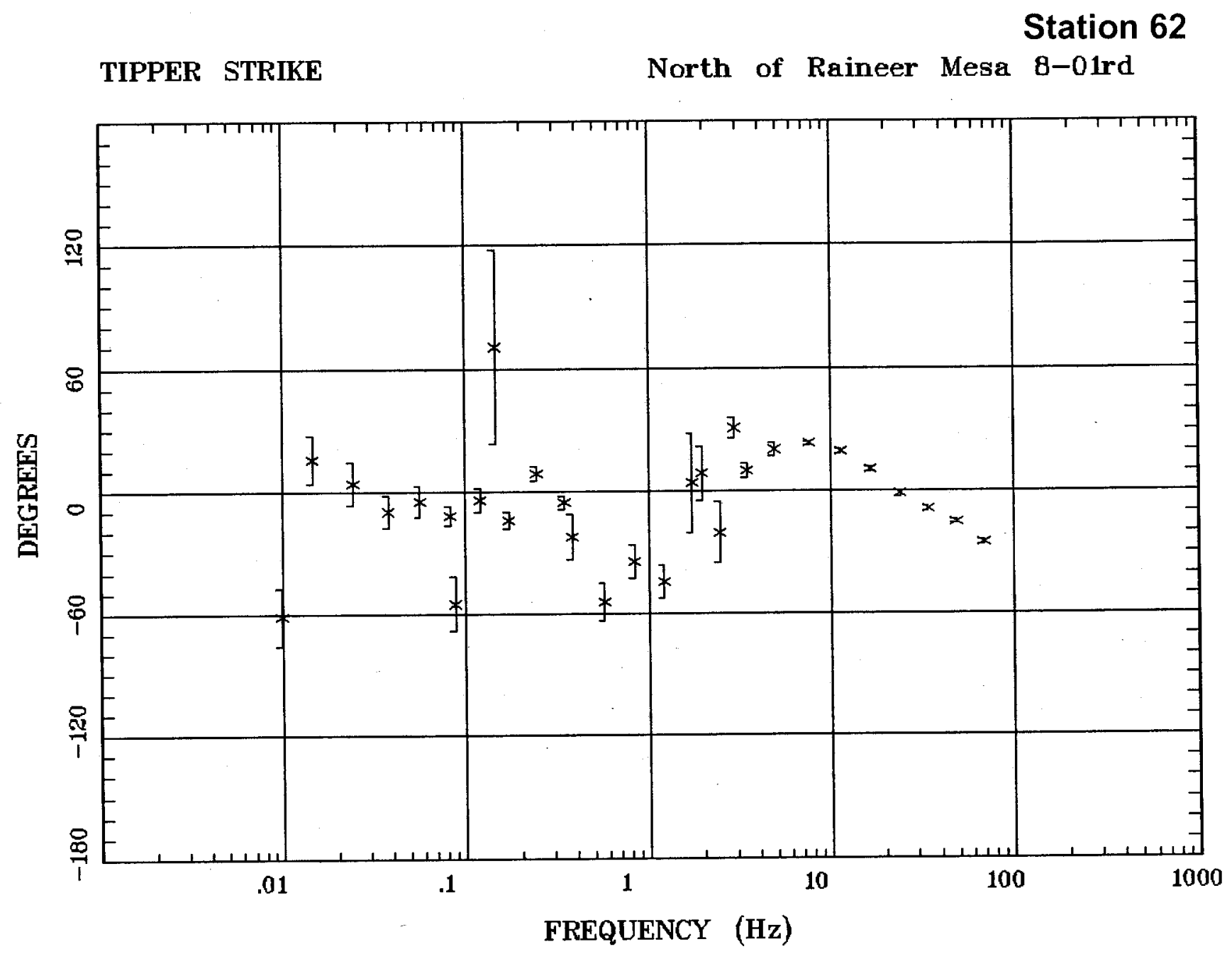

Client: WAter Resources \& DOD Remote: none

Acquired: 13:5 Feb 01, 2006 Survey Co:US Geological Survey

Ratation:

Filename: nt62n.evg

Channels: Ch1 Ch2 Ch3 Ch4 Ch5 Ch3 Ch4

Plotted: 15:19 Feb 08, 2006

< EMI - ElectroMagnetic Instruments > 


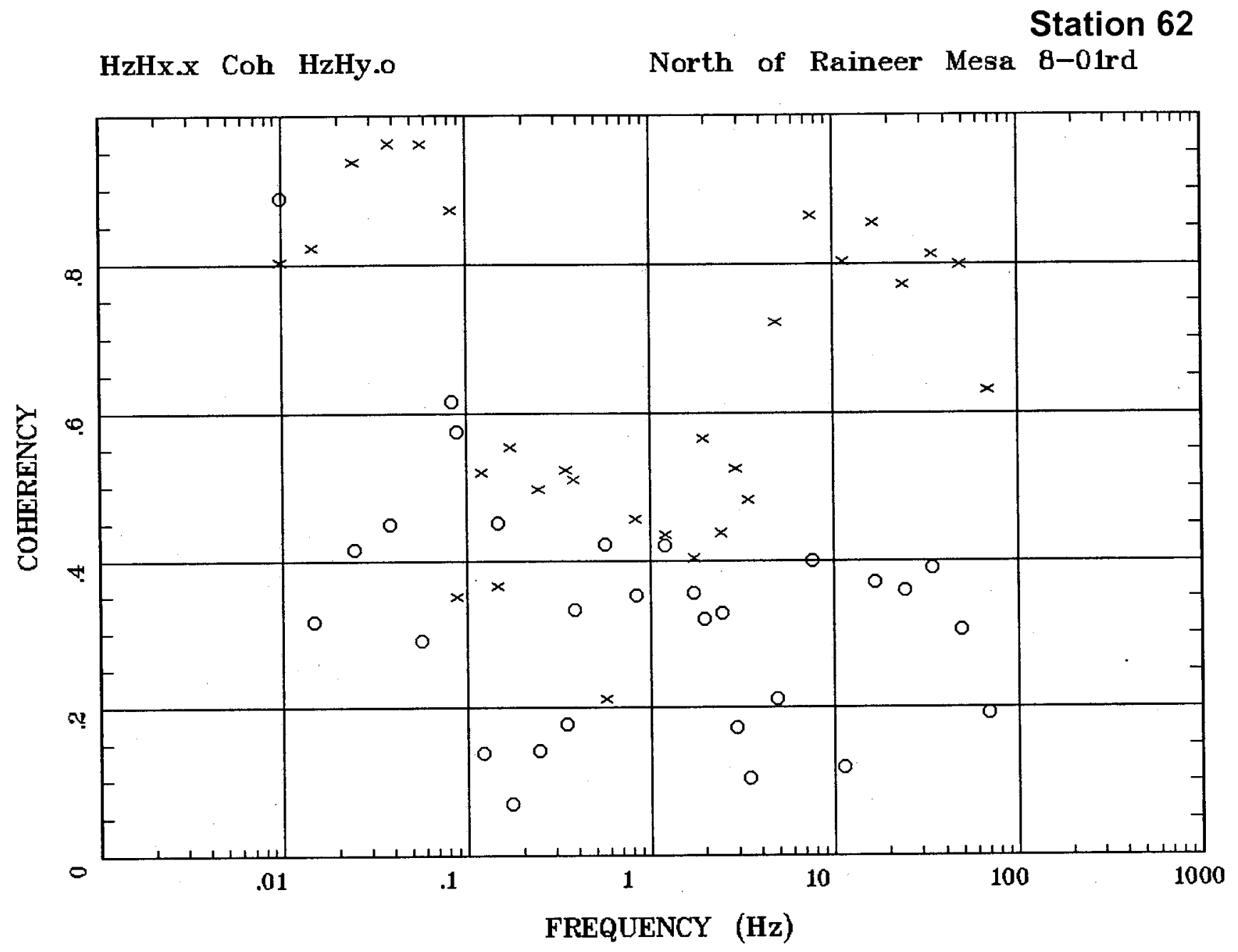

Client: WAter Resources \& DOD Remote: none Acquired: 13:5 Feb 01, 2006 Survey Co:US Geological Survey
Rotation:

Filename: nt62n.avg Channels: Ch1 Ch2 Ch3 Ch4 Ch5 Ch3 Ch4 Plotted: 15:19 Feb 08, 2006

< EMI - ElectroMagnetic Instruments > 


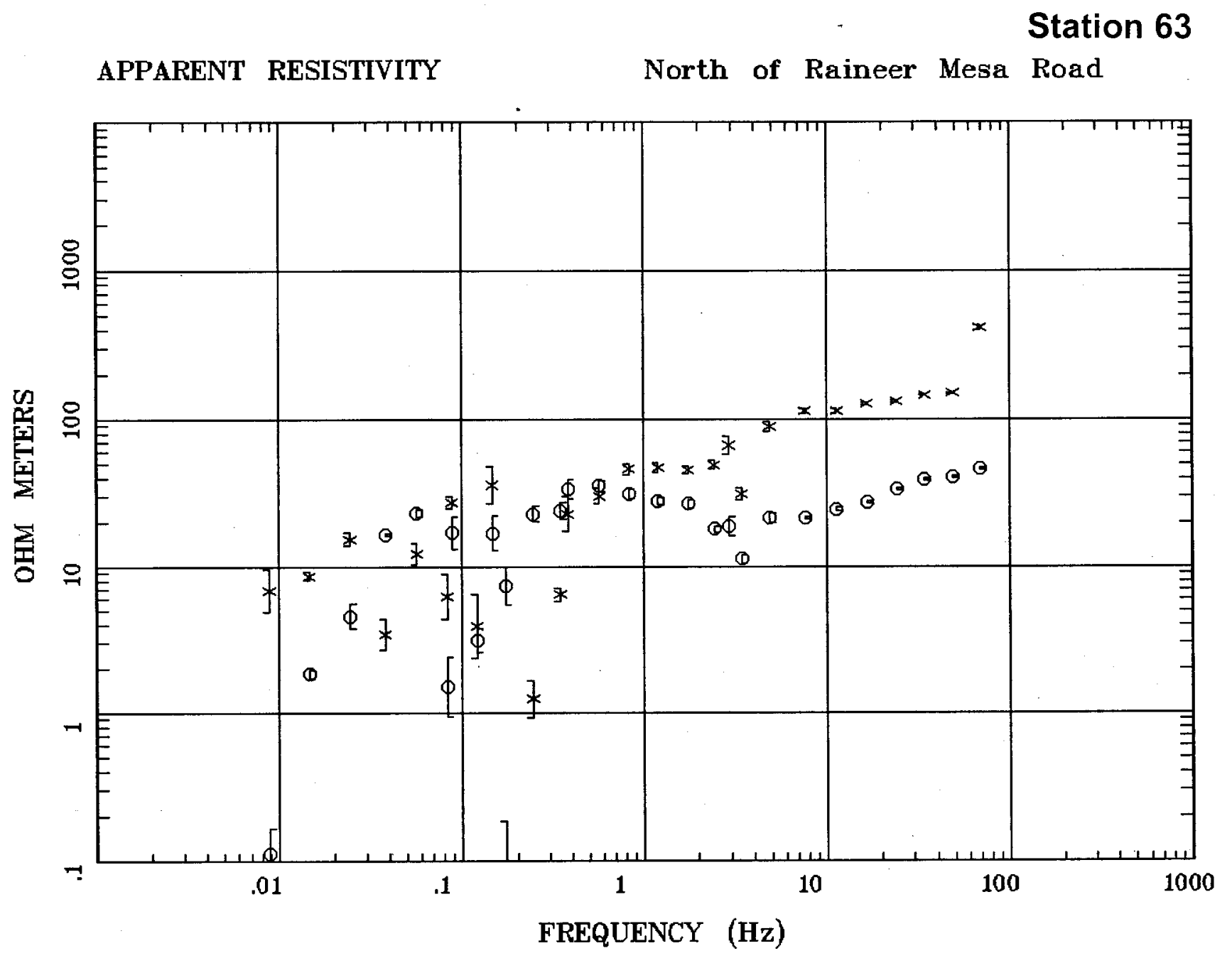

Client: FAter Resources \& DOD Remote: none Acquired: 05:3 oct 17, 2005 Survey Co:US Geological Survey
Rotation:

Filename: nt63m.avg Channels: Ch1 Ch2 Ch3 Ch4 Ch5 Ch3 Ch4 Plotted: 08:10 Feb 09, 2006

< EMI - ElectroMagnetic Instruments > 
IMPEDANCE PHASE

Station 63

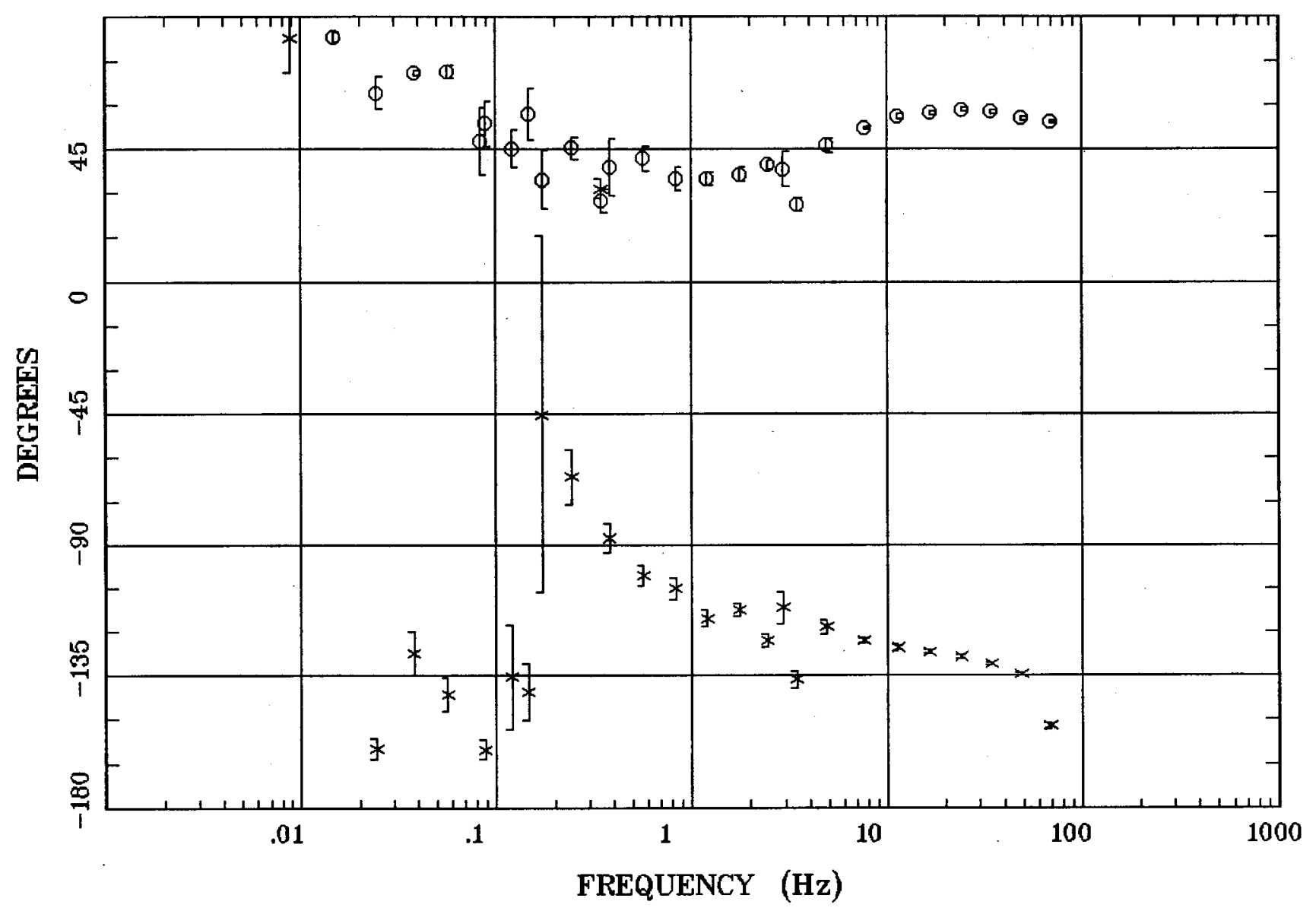

Client: WAter Resources \& DOD

Rotation:

Remote: none

Acquired: 05:3 oct 17, 2005

Survey Co:US Geological Survey

Filename: nt63m.avg

Channels: Ch1 Ch2 Ch3 Ch4 Ch5 Ch3 Ch4

Plotted: 08:10 Feb 09, 2006

< EMI - ElectroMagnetic Instruments > 
Station 63

ROTATION ANGLE

North of Raineer Mesa Road

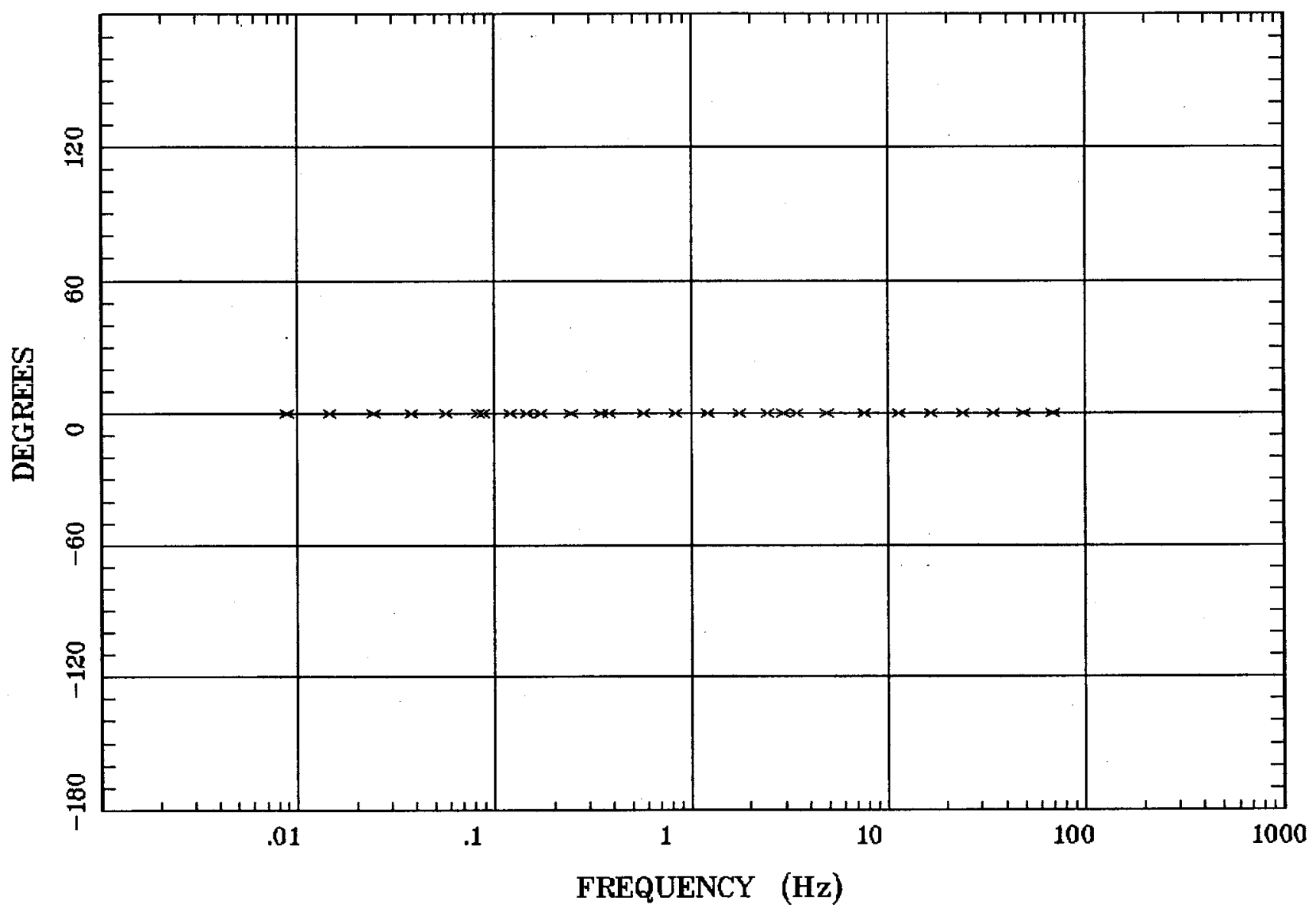

Client: WAter Resources \& DOD

Remote: none

Acquired: 05:3 oct 17, 2005

Survey Co:US Geological Survey

Rotation:

Filename: nt63m.avg

Channels: Ch1 Ch2 Ch3 Ch4 Ch5 Ch3 Ch4

Plotted: 08:10 Feb 09, 2006

< EMI - ElectroMagnetic Instruments > 


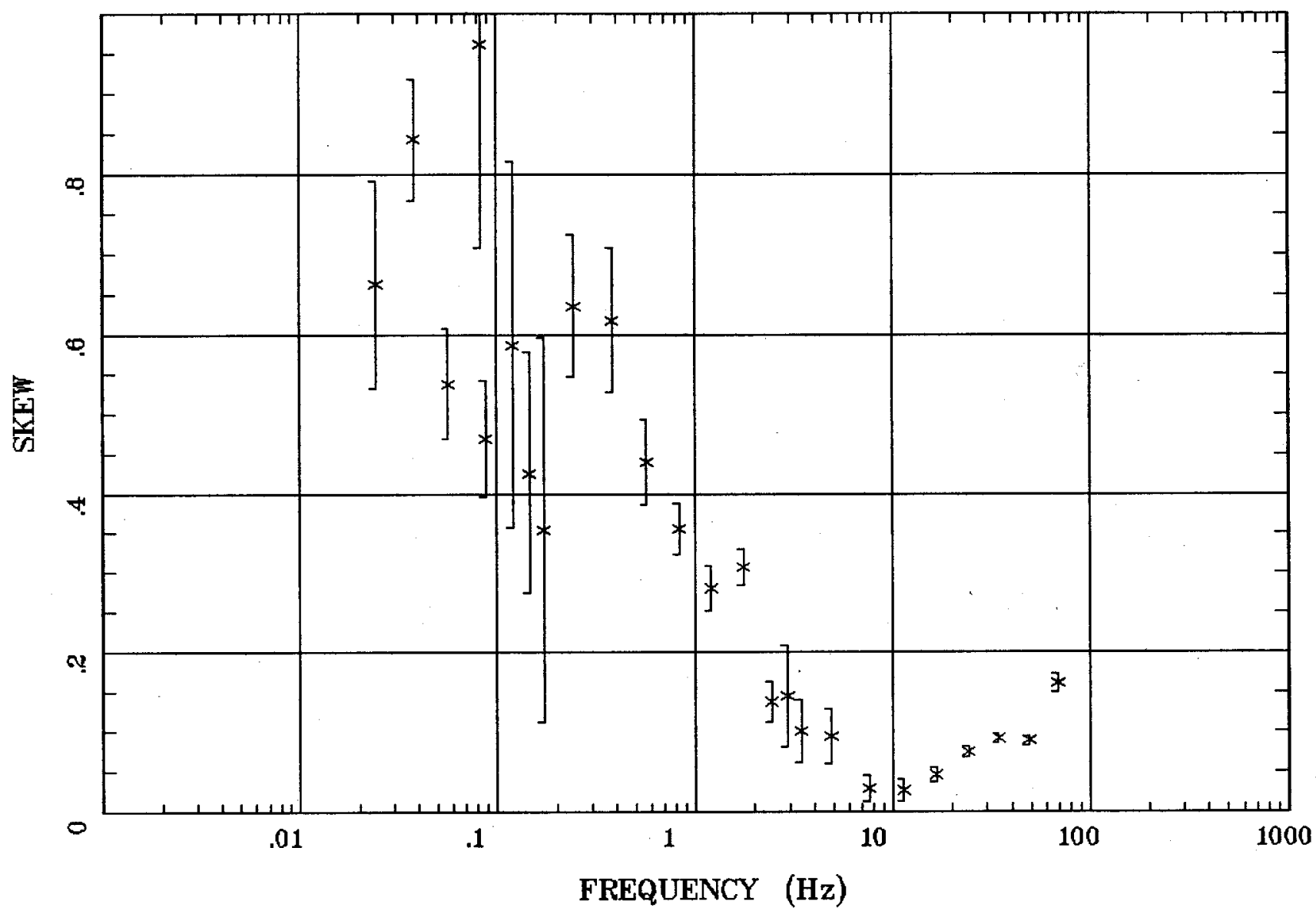

Client: WAter Resources \& DOD Remote: none Acquired: 05:3 Oct 17, 2005 Survey Co:US Geological Survey
North of Raineer Mesa Road

Rotation:

Filename: nt63m.avg

Channels: Ch1 Ch2 Ch3 Ch4 Ch5 Ch3 Ch4

Plotted: 08:10 Feb 09, 2006

< EMI - ElectroMagnetic Instruments > 
Station 63

E MULT Coh.

North of Raineer Mesa Road

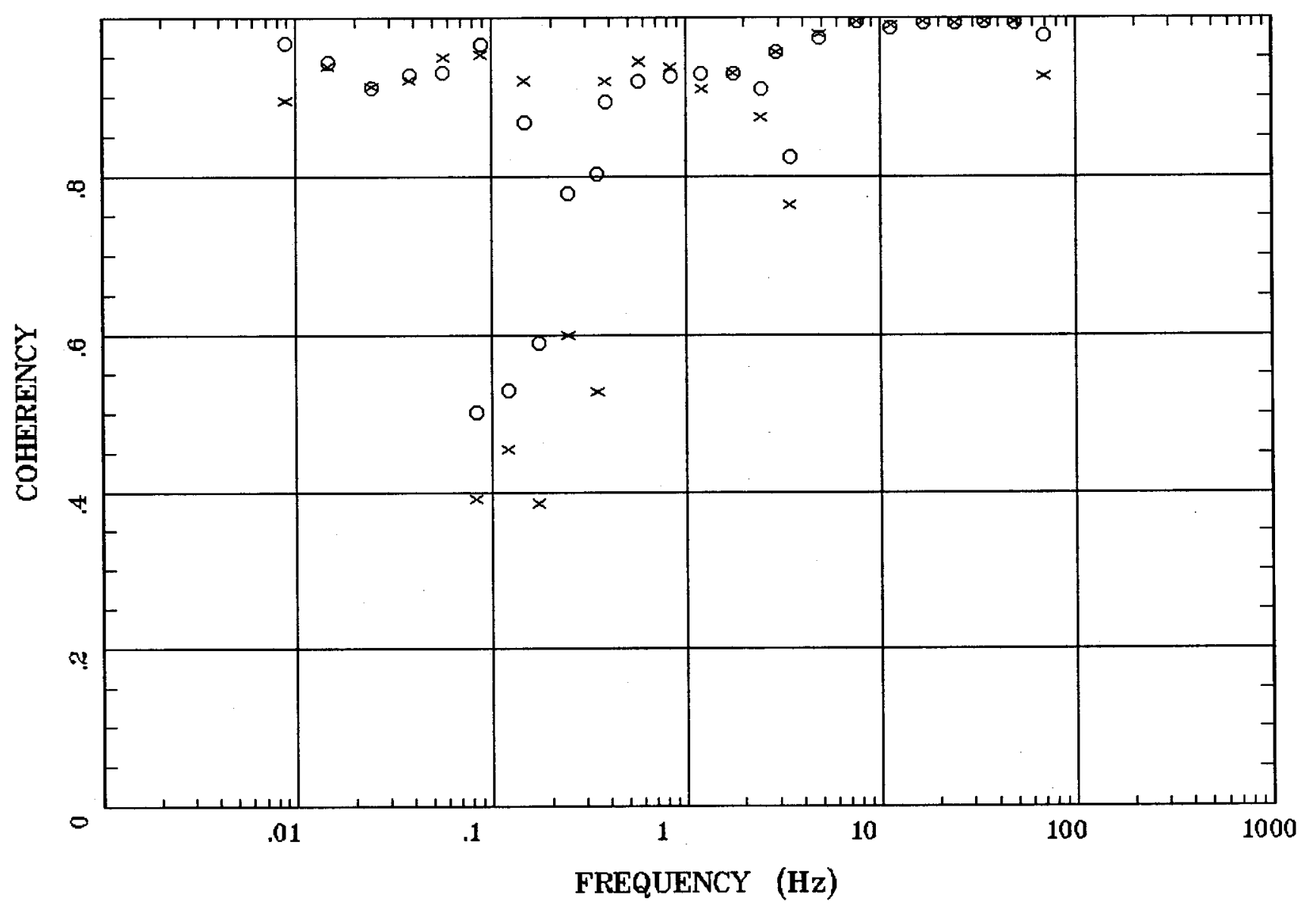

Client: WAter Resources \& DOD Remote: none Acquired: $05: 3$ oct 17, 2005 Survey Co:US Geological Survey

Rotation:

Filename: nt63m.avg Channels: Ch1 Ch2 Ch3 Ch4 Ch5 Ch3 Ch4 Plotted: 08:10 Feb 09, 2006

< EMI - ElectroMagnetic Instruments > 


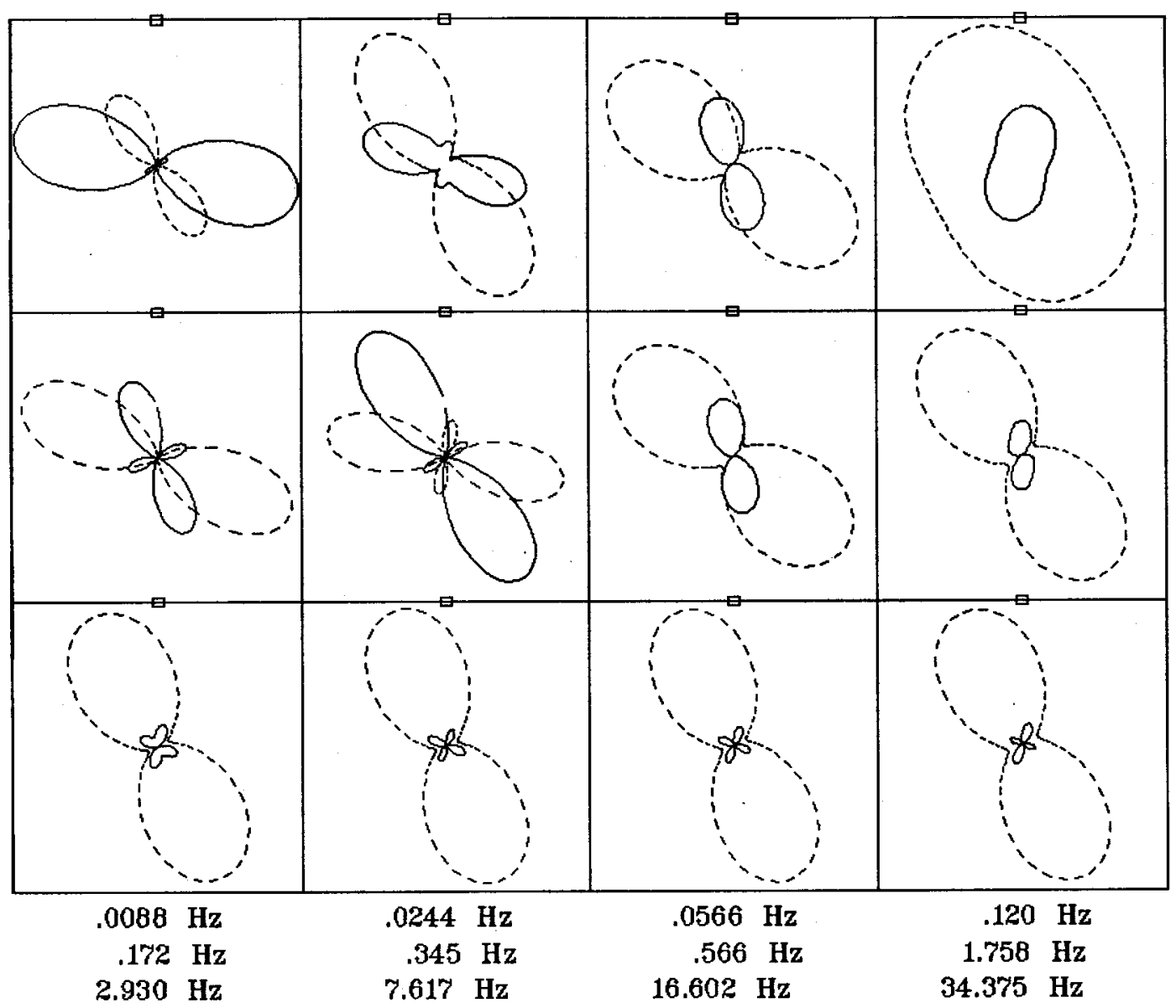

Client: WAter Resources \& DOD Remote: none

Acquired: 05:3 oct 17, 2005 Survey Co:US Geological Survey
Rotation:

Filename: nt63m.avg

Channels: Ch1 Ch2 Ch3 Ch4 Ch5 Ch3 Ch4

Plotted: 08:10 Feb 09, 2006

< EMI - ElectraMagnetic Instruments > 
TIPPER MAGNITUDE

Station 63

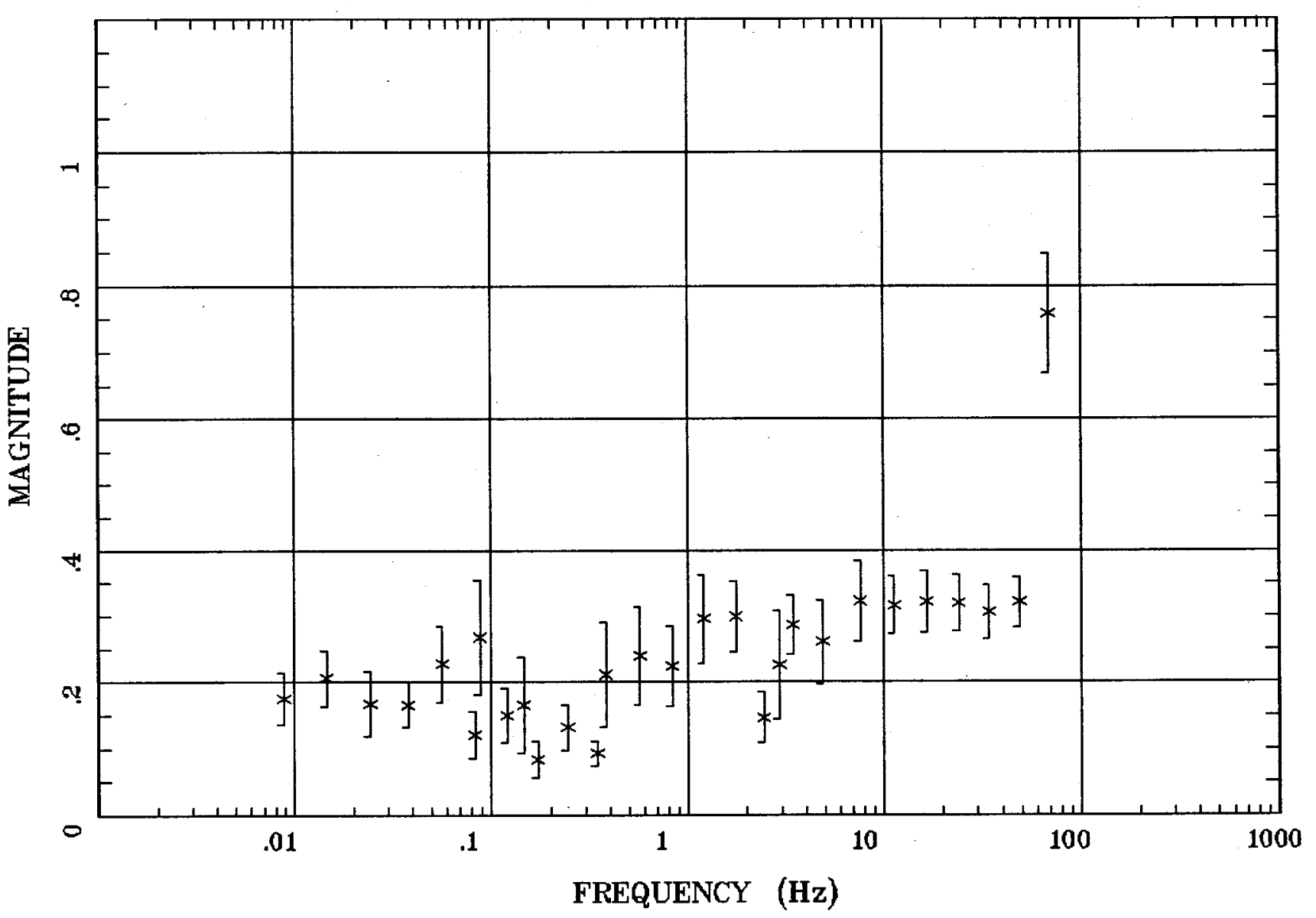

Client: WAter Resources \& DOD Remote: none Acquired: $05: 3$ act 17, 2005 Survey Co:US Geological Survey

Rotation:

Filename: nt63m.avg

Channels: Ch1 Ch2 Ch3 Ch4 Ch5 Ch3 Ch4

Platted: 08:10 Feb 09, 2006

< EMI - ElectroMagnetic Instruments > 
Station 63

TIPPER STRIKE

North of Raineer Mesa Road

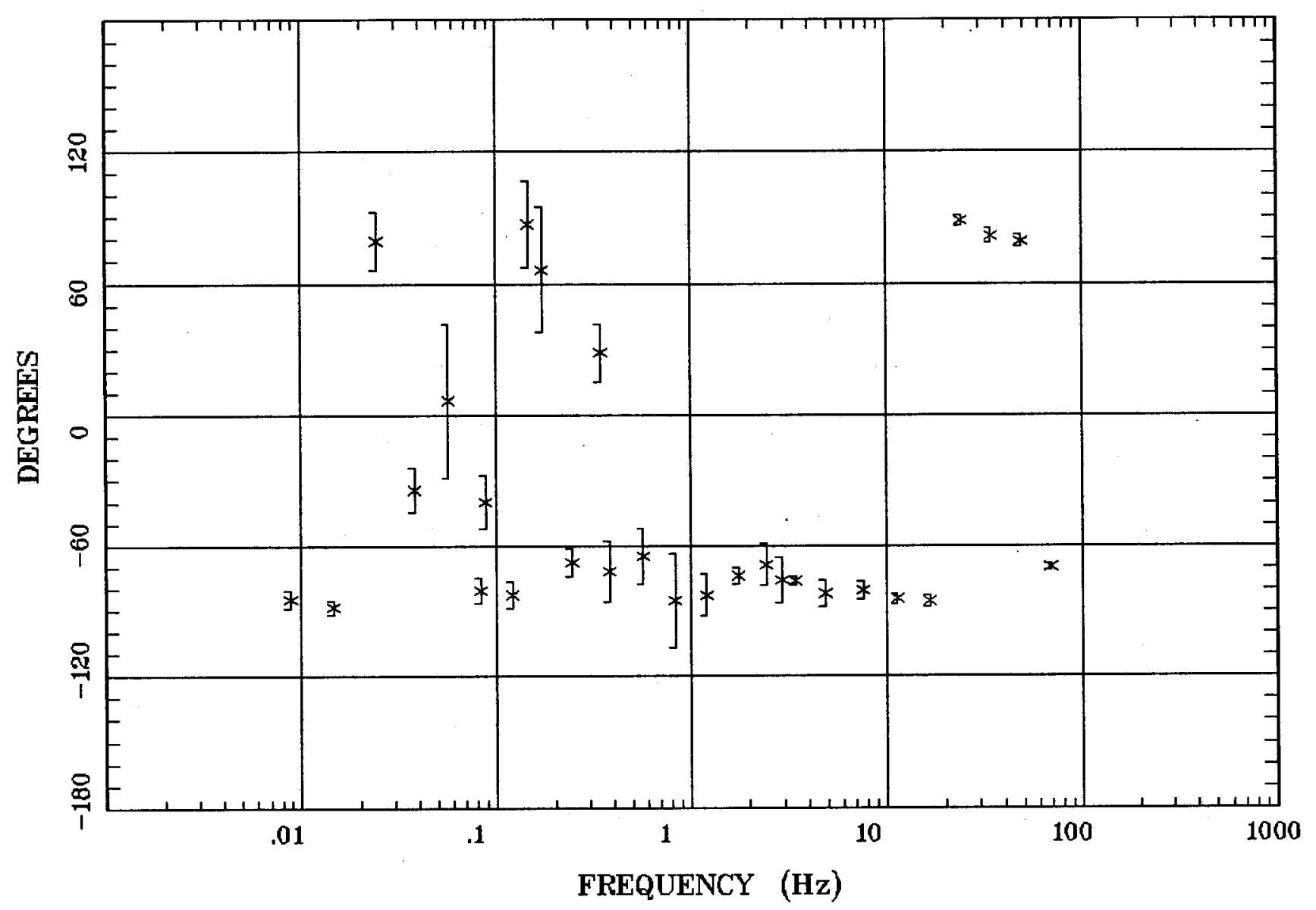

Client: WAter Resources \& DOD Remote: none Acquired: 05:3 oct 17, 2005 Survey Co:US Geological Survey
Rotation:

Filename: nt63m.avg Channels: Ch1 Ch2 Ch3 Ch4 Ch5 Ch3 Ch4 Plotted: $08: 10$ Feb 09, 2006

< EMI - ElectroMagnetic Instruments > 
HzHx.x Coh HzHy.o

Station 63

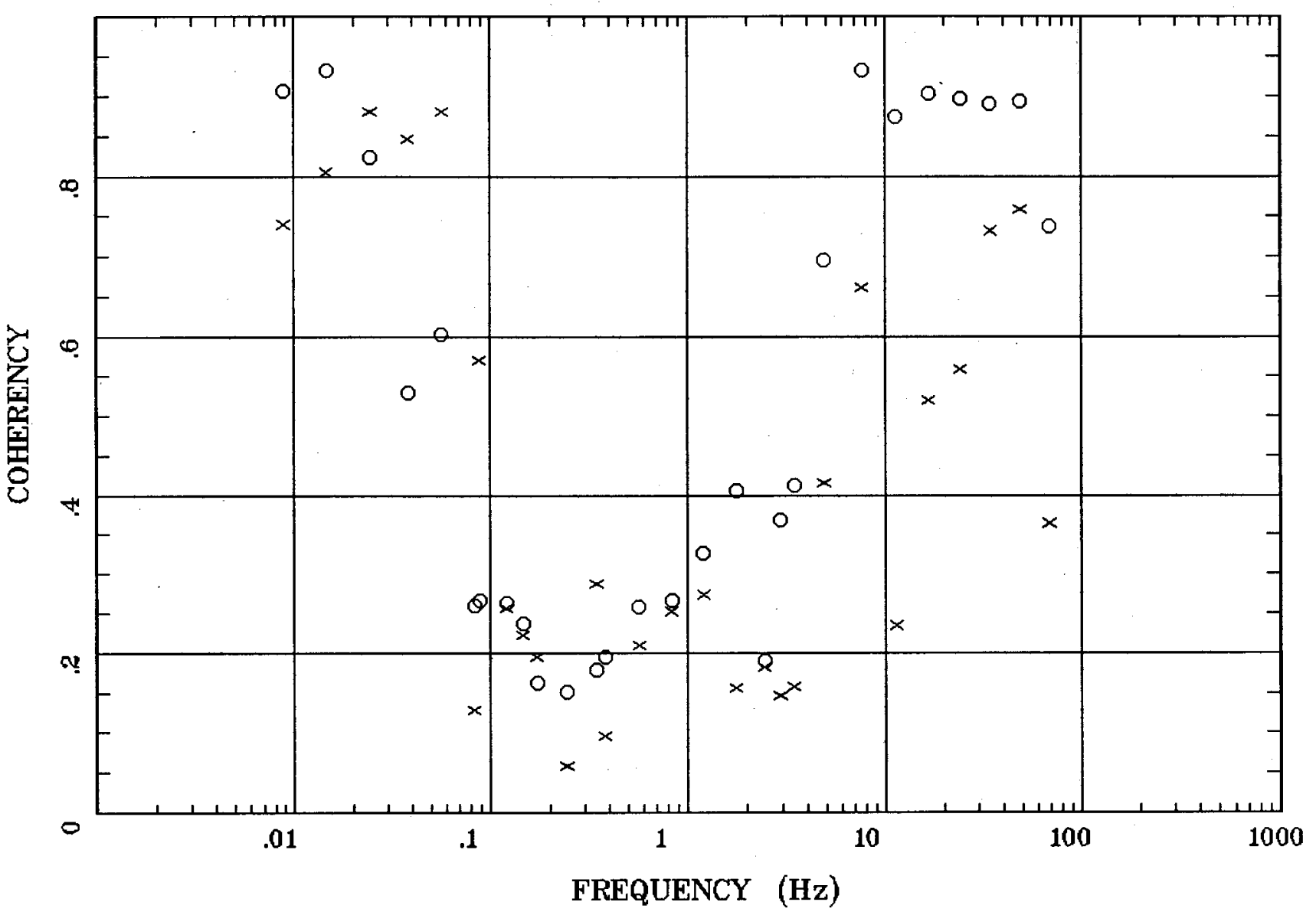

Client: WAter Resources \& DOD

Rotation:

Filename: nt69m.avg

Channels: Ch1 Ch2 Ch3 Ch4 Ch5 Ch3 Ch4

Plotted: 08:10 Feb 09, 2006

Acquired: $05: 3$ oct 17,2005

Survey Co:US Geological Survey

< EMI - ElectroMagnetic Instruments > 


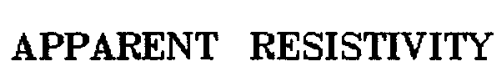

Station 64

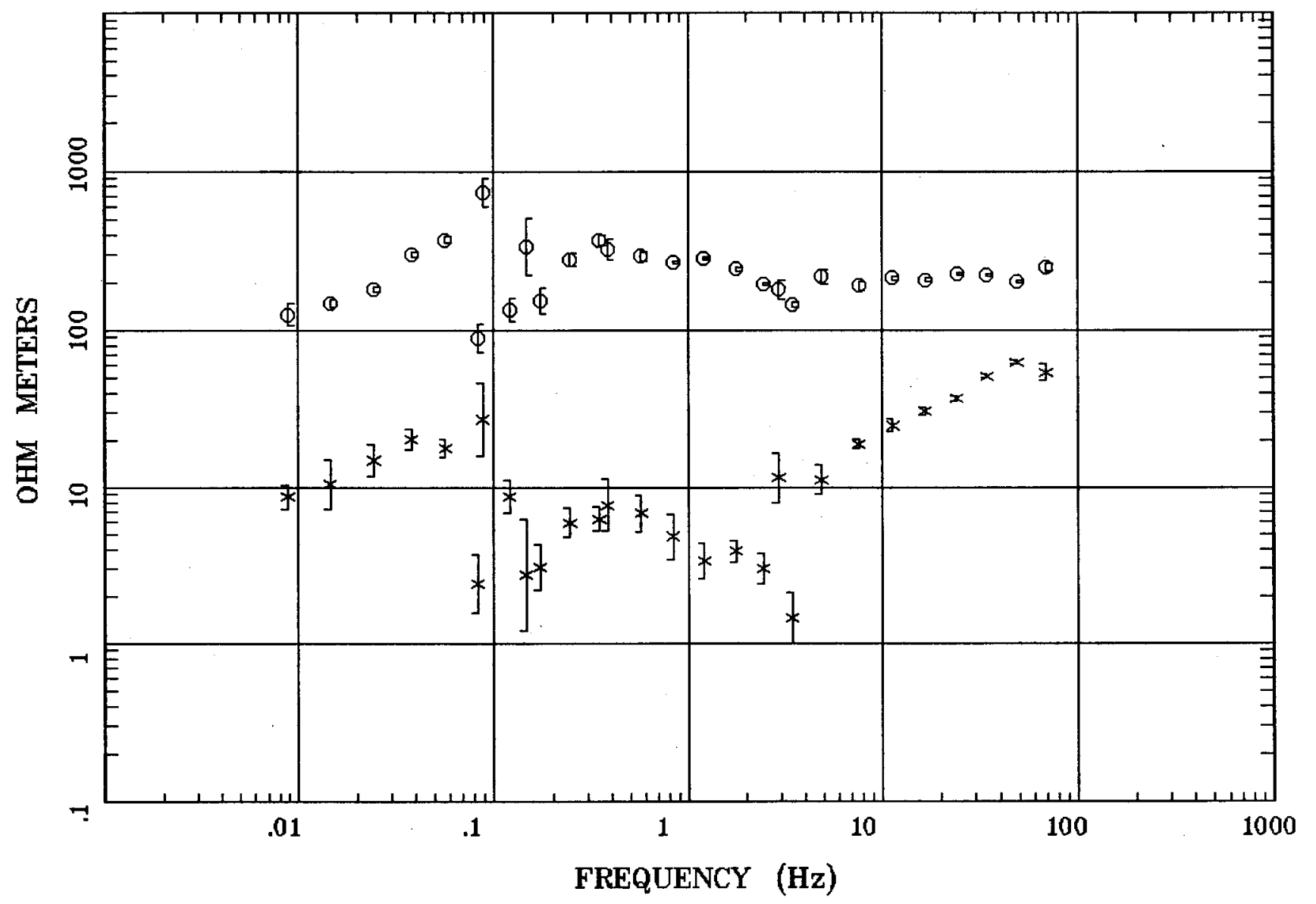

Client: FAter Resources \& DOD Remote: none Acquired: 10:4 Oct 18, 2005 Survey Co:US Geological Survey
North of Raineer Mesa Road

Rotation:

Filename: nt64m.avg

Channels: Ch1 Ch2 Ch3 Ch4 Ch5 Ch3 Ch4

Plotted: 10:19 Feb 09, 2006.

< EMI - ElectroMagnetic Instruments > 


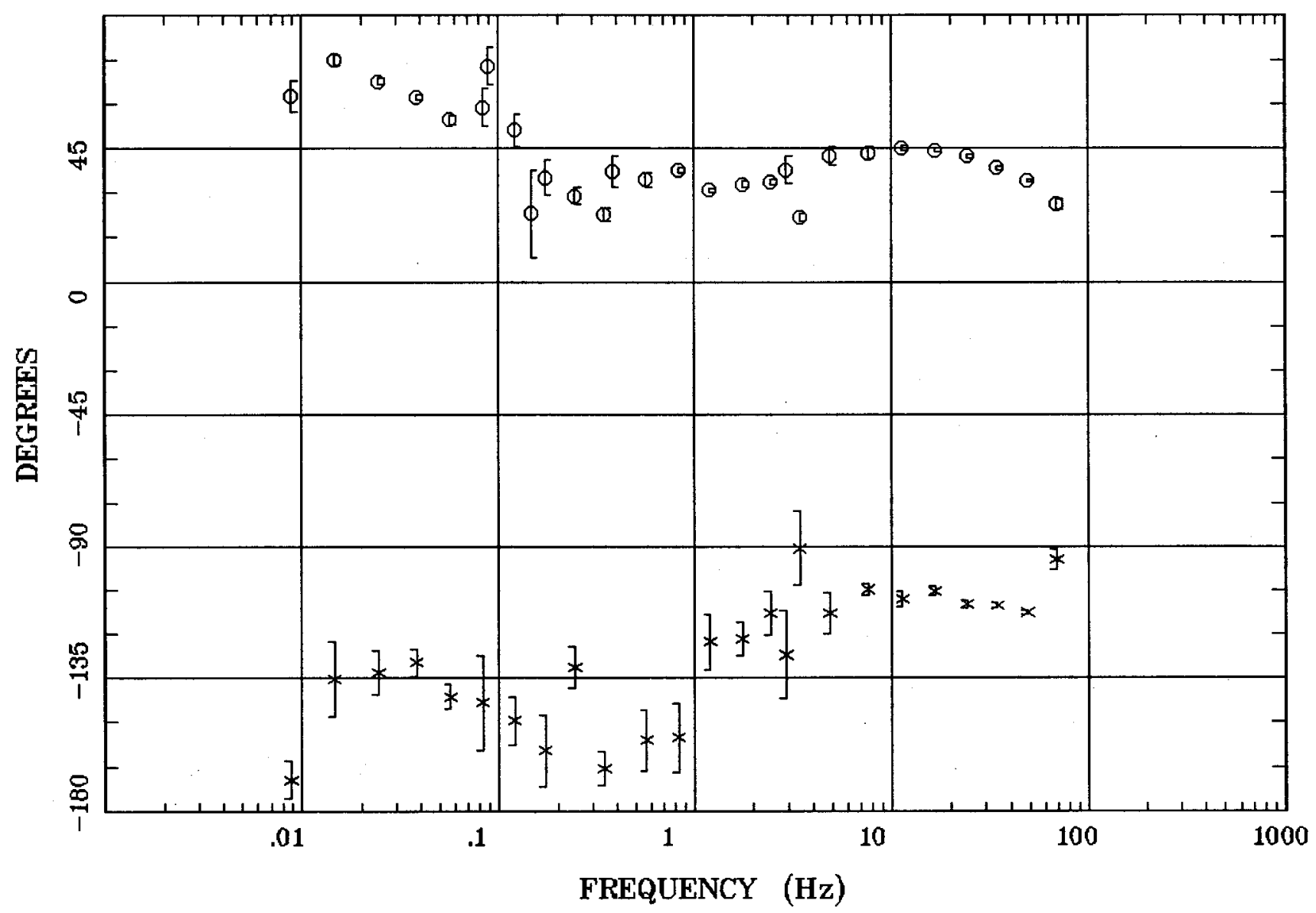

Client: WAter Resources \& DOD Remote: none Acquired: 10:4 Oct 18, 2005 Survey Co:US Geological Survey
Rotation:

Fileneme: nt64m.avg

Channels: Ch1 Ch2 Ch3 Ch4 Ch5 Ch3 Ch4 Plotted: 10:19 Feb 09, 2006

< EMI - ElectroMagnetic Instruments > 


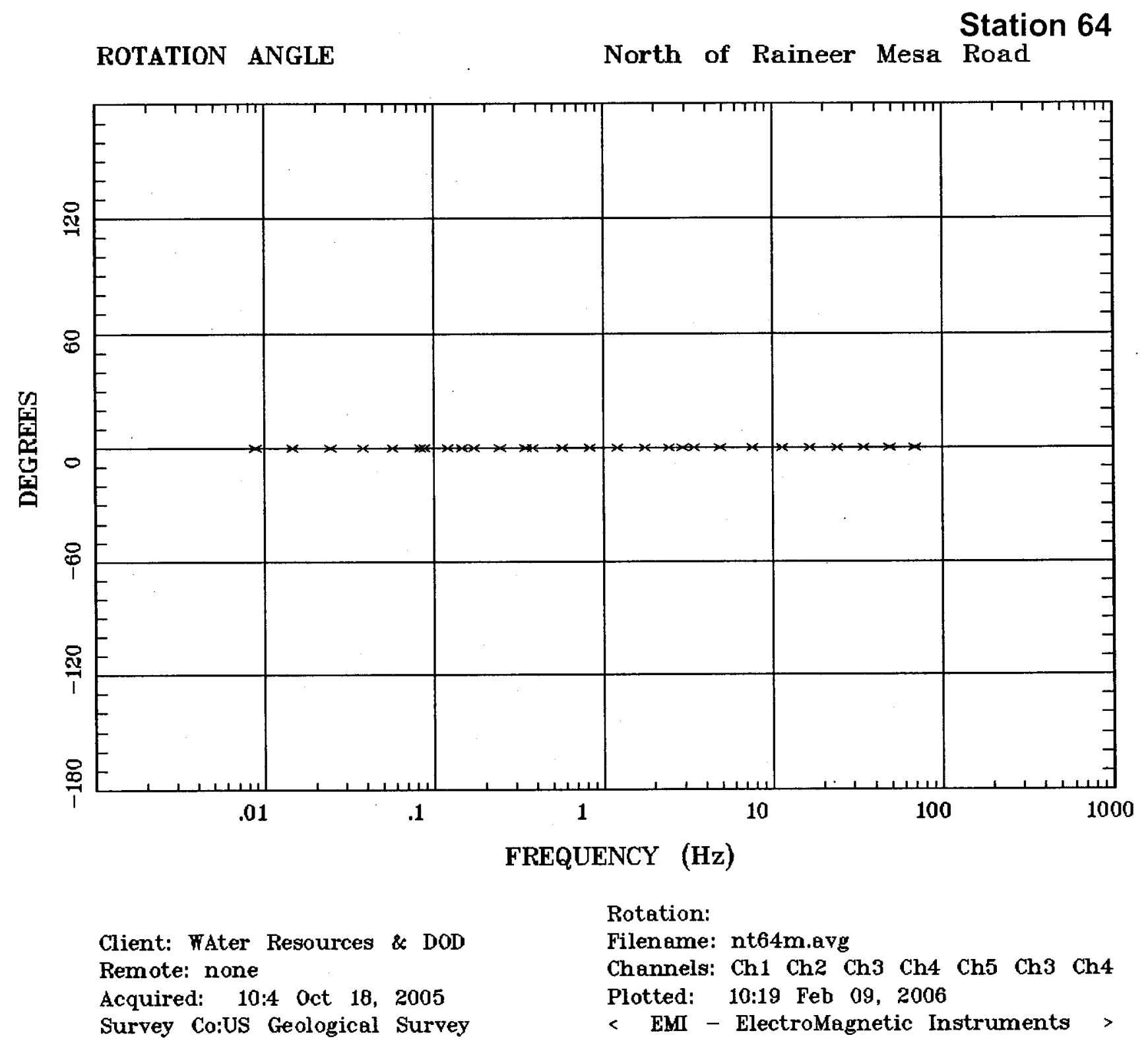


Station 64

IMPEDANCE SKEW

North of Raineer Mesa Road

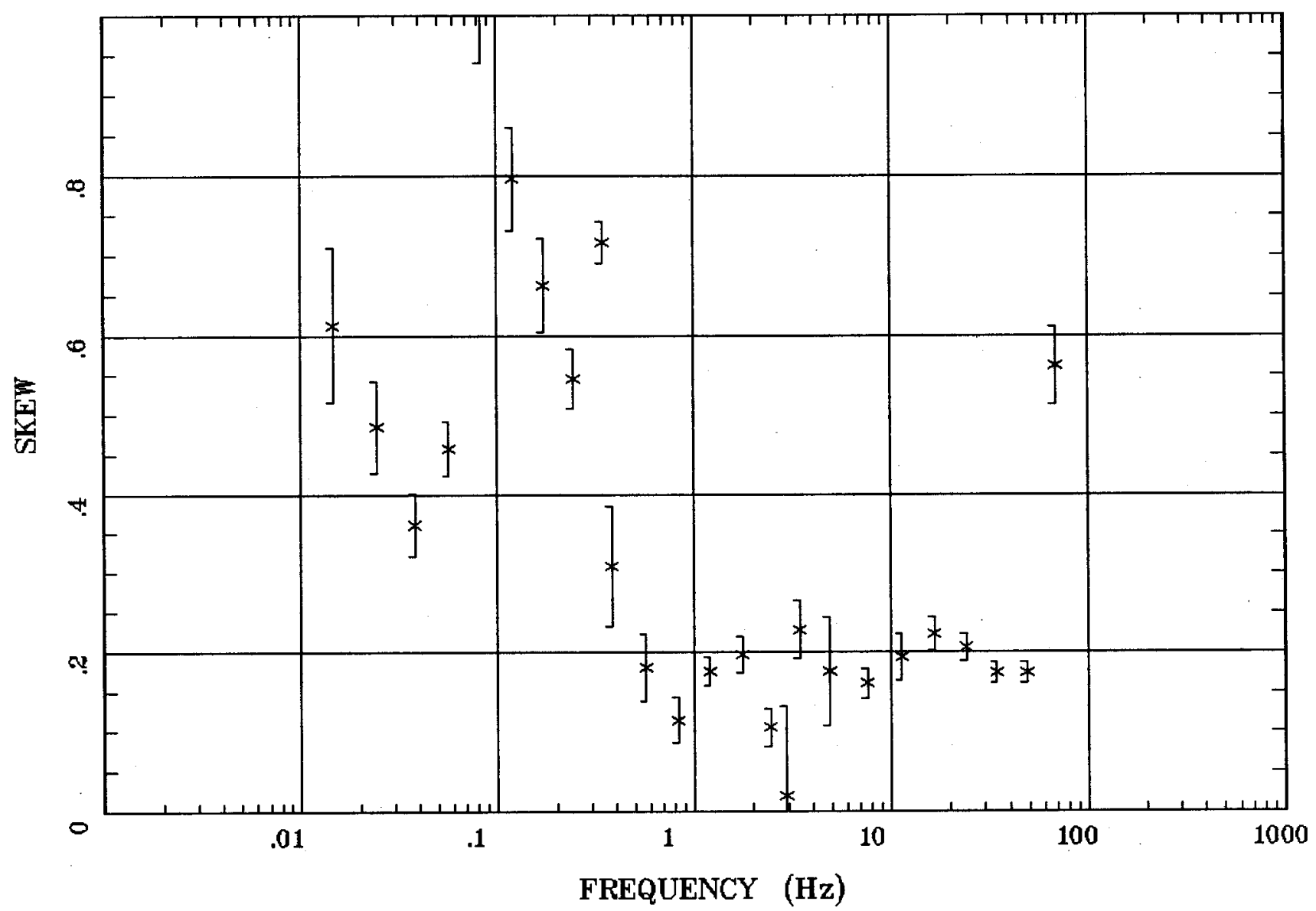

Client: WAter Resources \& DOD Remote: none

Acquired: 10:4 Oct 18, 2005 Survey Co:US Geological Survey
Rotation:

Filename: nt64m.avg

Channels: Ch1 Ch2 Ch3 Ch4 Ch5 Ch3 Ch4 Plotted: 10:19 Feb 09, 2006

< EMI - ElectroMagnetic Instruments > 
Station 64

E MULT Coh.

North of Raineer Mesa Road

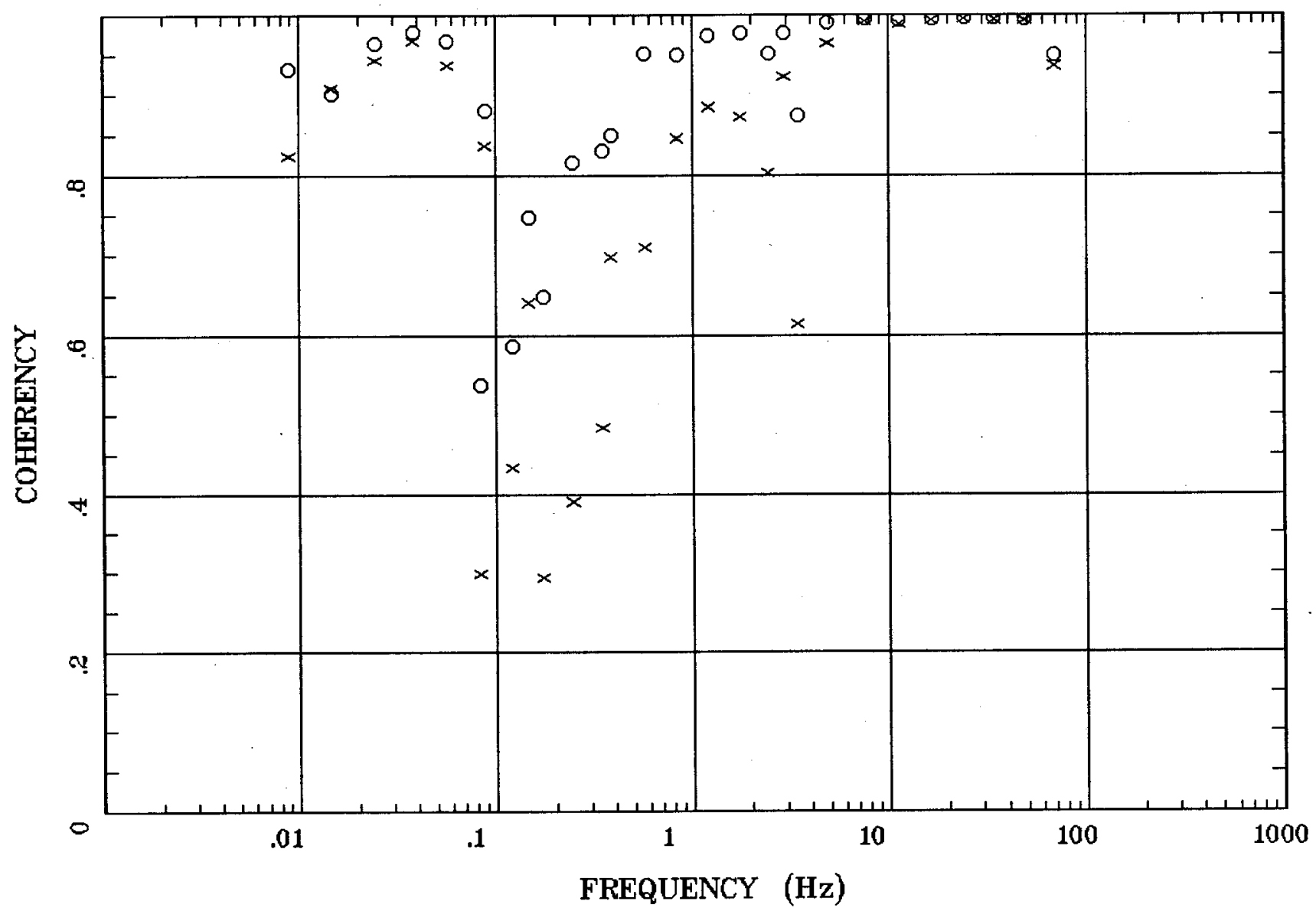

Client: WAter Resources \& DOD Remote: none

Acquired: 10:4 Oct 18, 2005

Survey Co:US Geological Survey

Rotation:

Filename: nt64m.avg

Channels: Ch1 Ch2 Ch3 Ch4 Ch5 Ch3 Ch4

Plotted: 10:19 Feb 09, 2006

< EMI - ElectroMagnetic Instruments > 


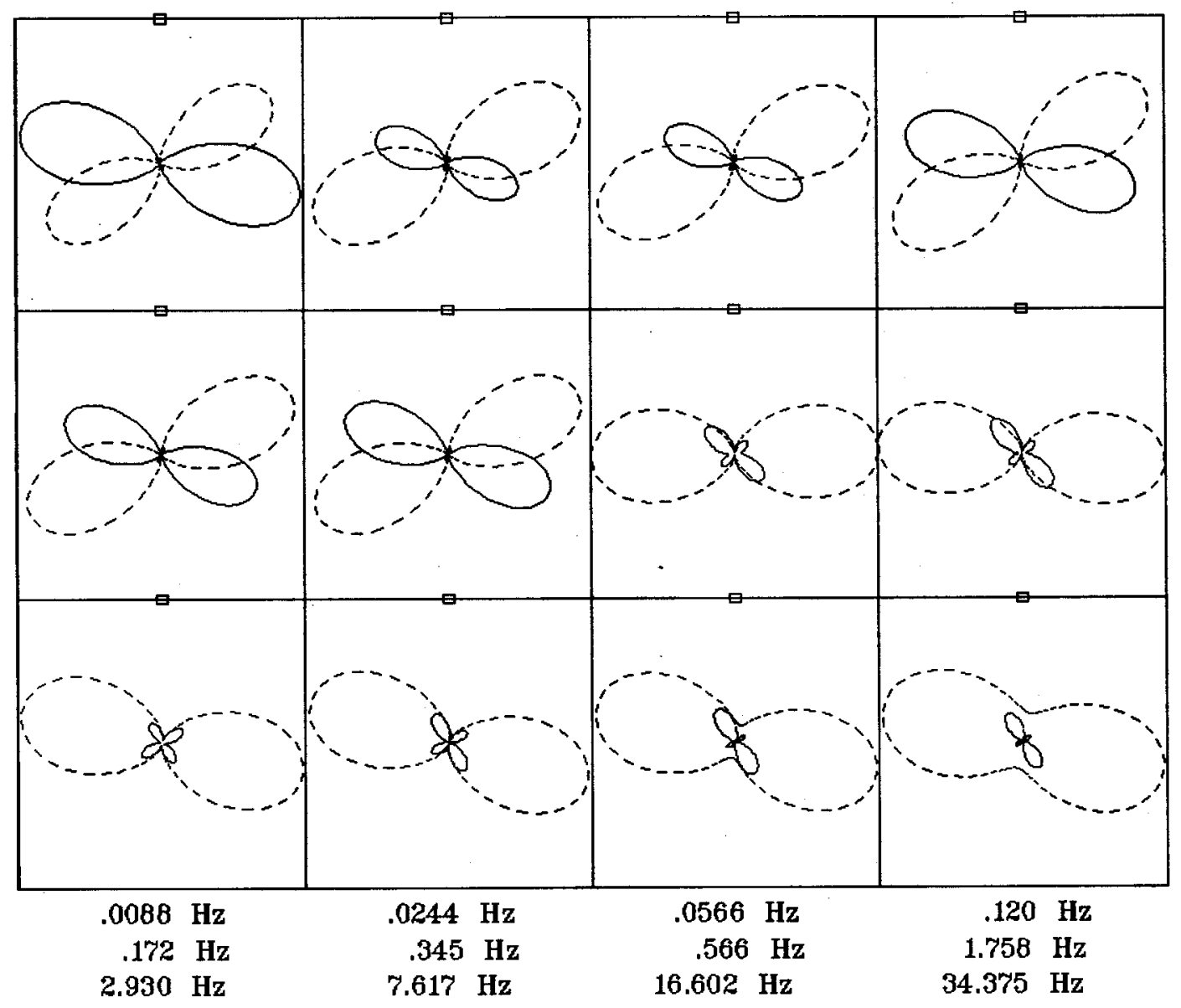

Client: FAter Resources \& DOD Remote: none

Acquired: 10:4 oct 18, 2005 Survey Co:US Geological Survey

Rotation:

Filename: nt64m.avg

Channels: Ch1 Ch2 Ch3 Ch4 Ch5 Ch3 Ch4

Platted: 10:19 Feb 09, 2006

< EMI - ElectroMagnetic Instruments > 
TIPPER MAGNITUDE

Station 64

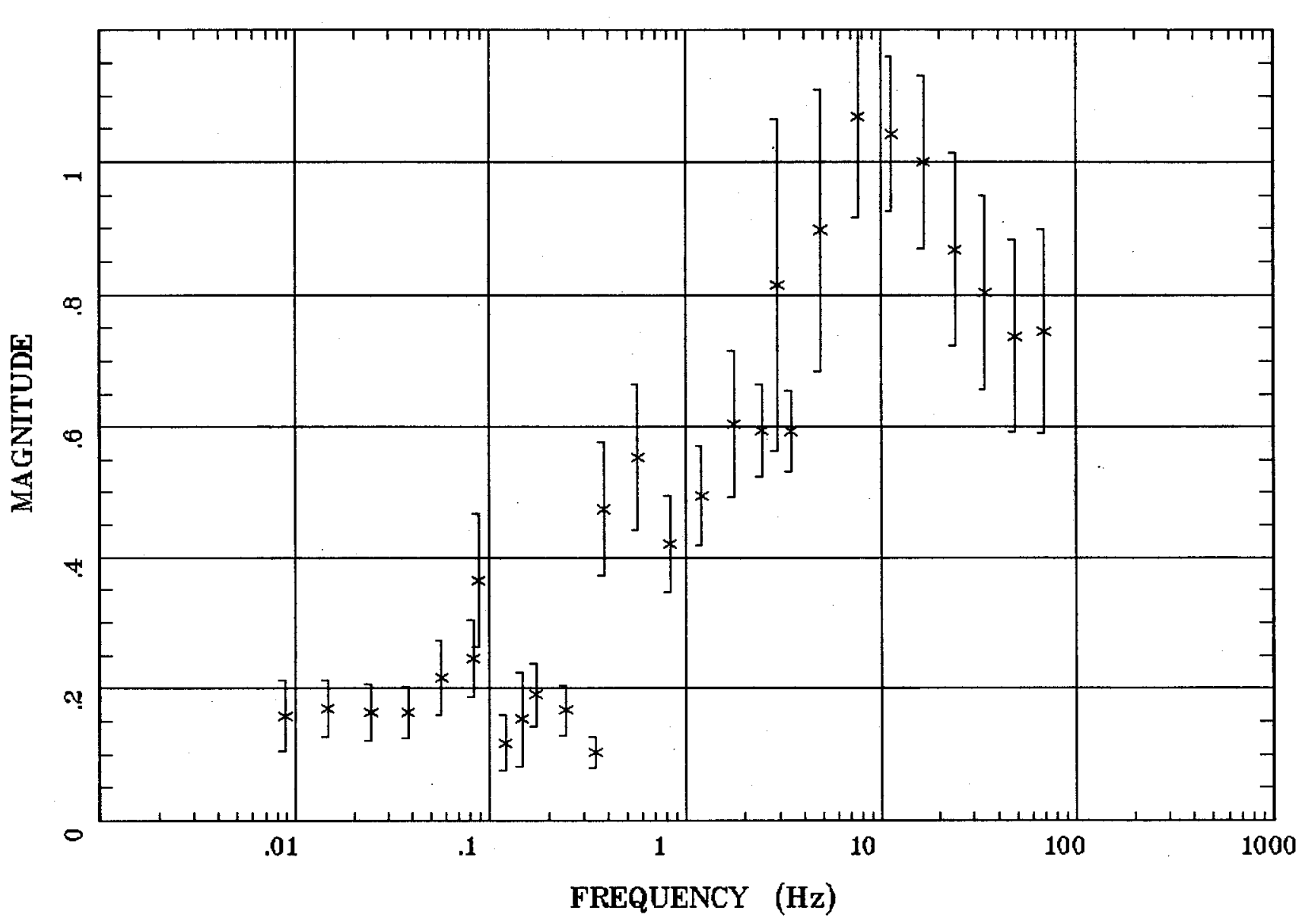

Client: WAter Resources \& DOD

Rotation:

Remote: none

Acquired: 10:4 Oct 18, 2005

Survey Co:US Geological Survey

Filename: nt64m.avg

Channels: Ch1 Ch2 Ch3 Ch4 Ch5 Ch3 Ch4

Plotted: 10:19 Feb 09, 2006

< EMI - ElectroMagnetic Instruments > 


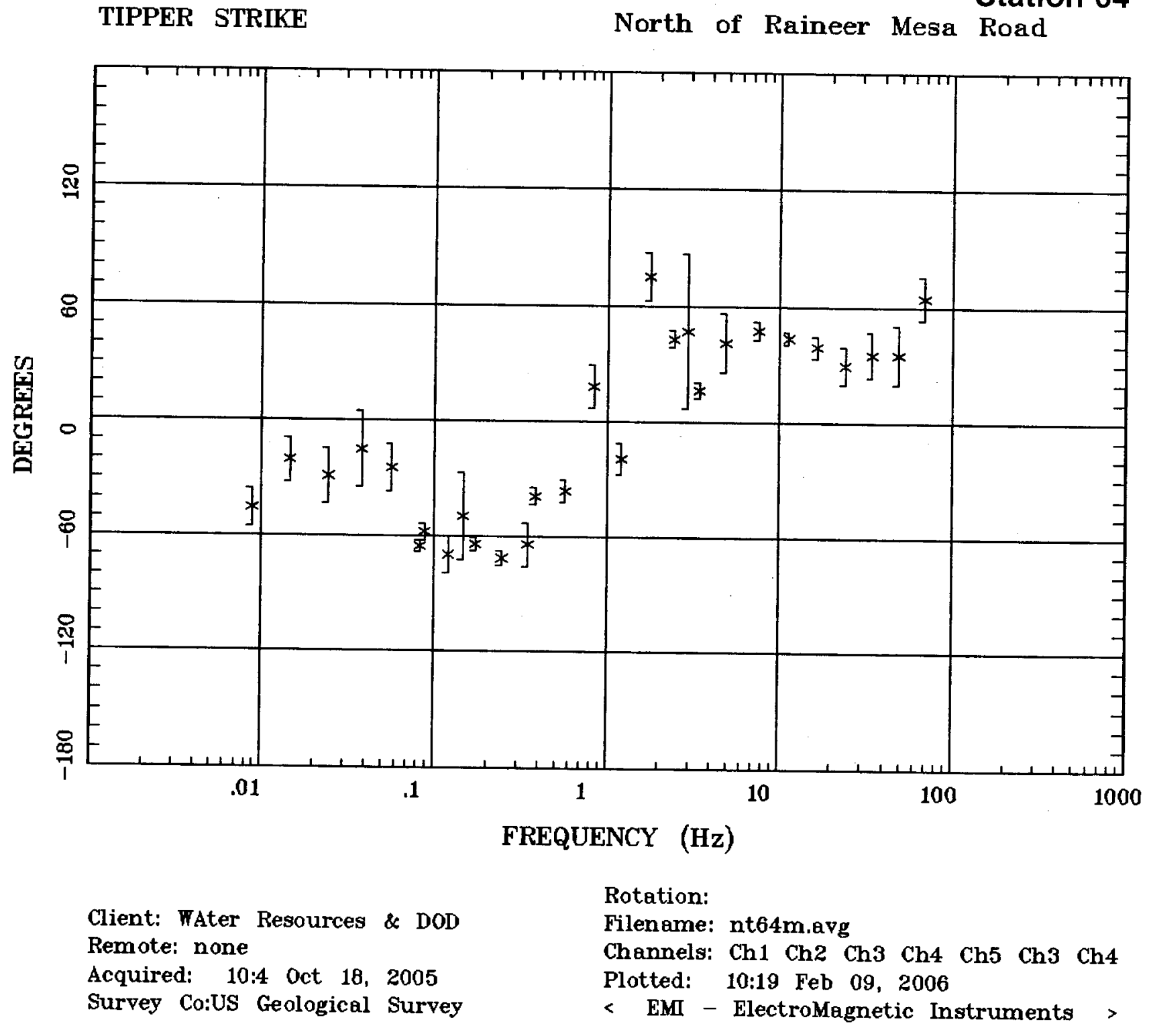


HzHx.x Coh HzHy.o

Station 64

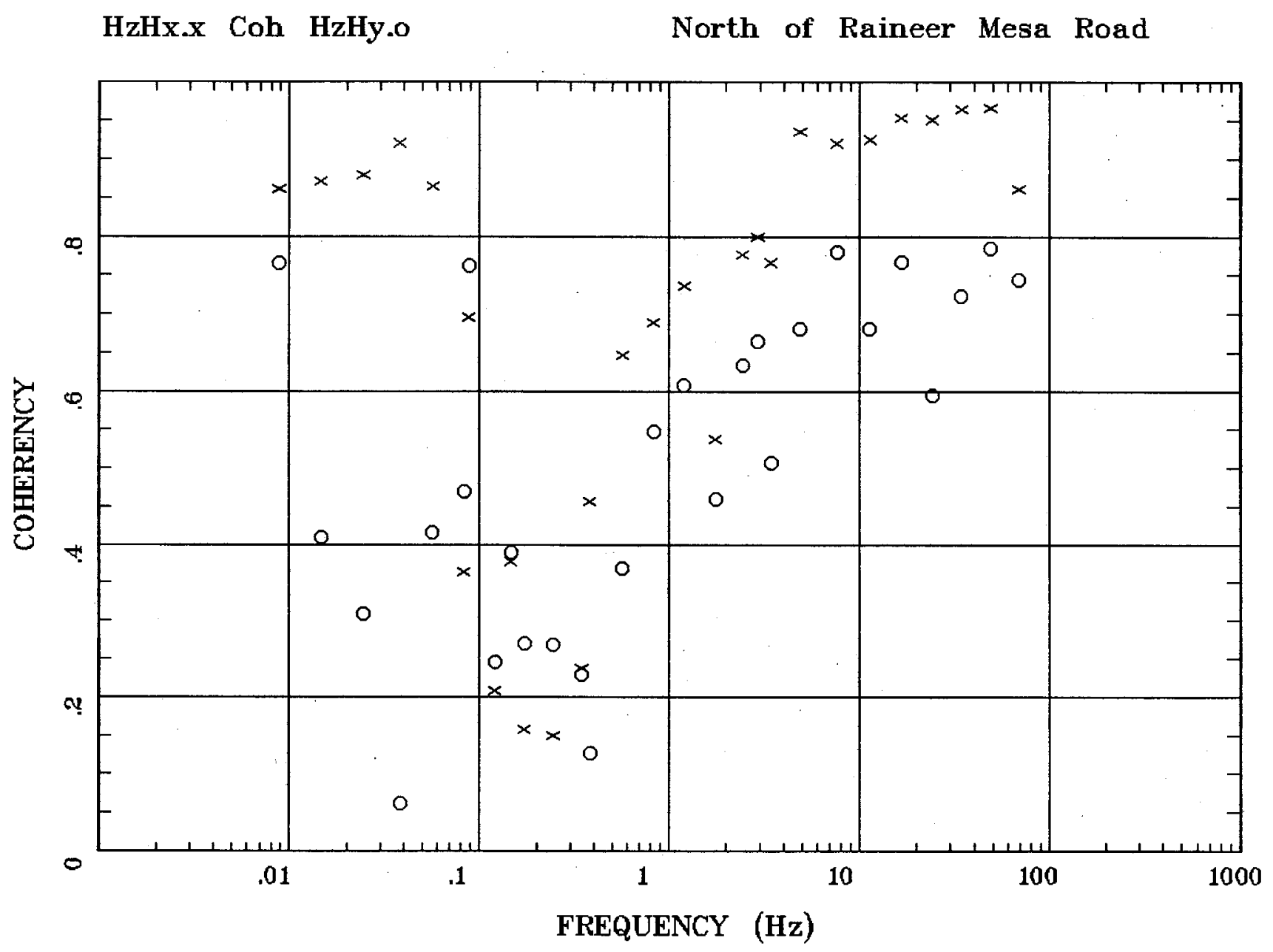

Client: FAter Resources \& DOD Remote: none

Acquired: 10:4 Oct 18, 2005 Survey Co:US Geological Survey
Rotation:

Filename: nt64m.avg Channels: Ch1 Ch2 Ch3 Ch4 Ch5 Ch3 Ch4 Plotted: 10:19 Feb 09, 2006

< EMI - ElectroMagnetic Instruments > 
Station 65

APPARENT RESISTIVITY

North of Raineer Mesa Road

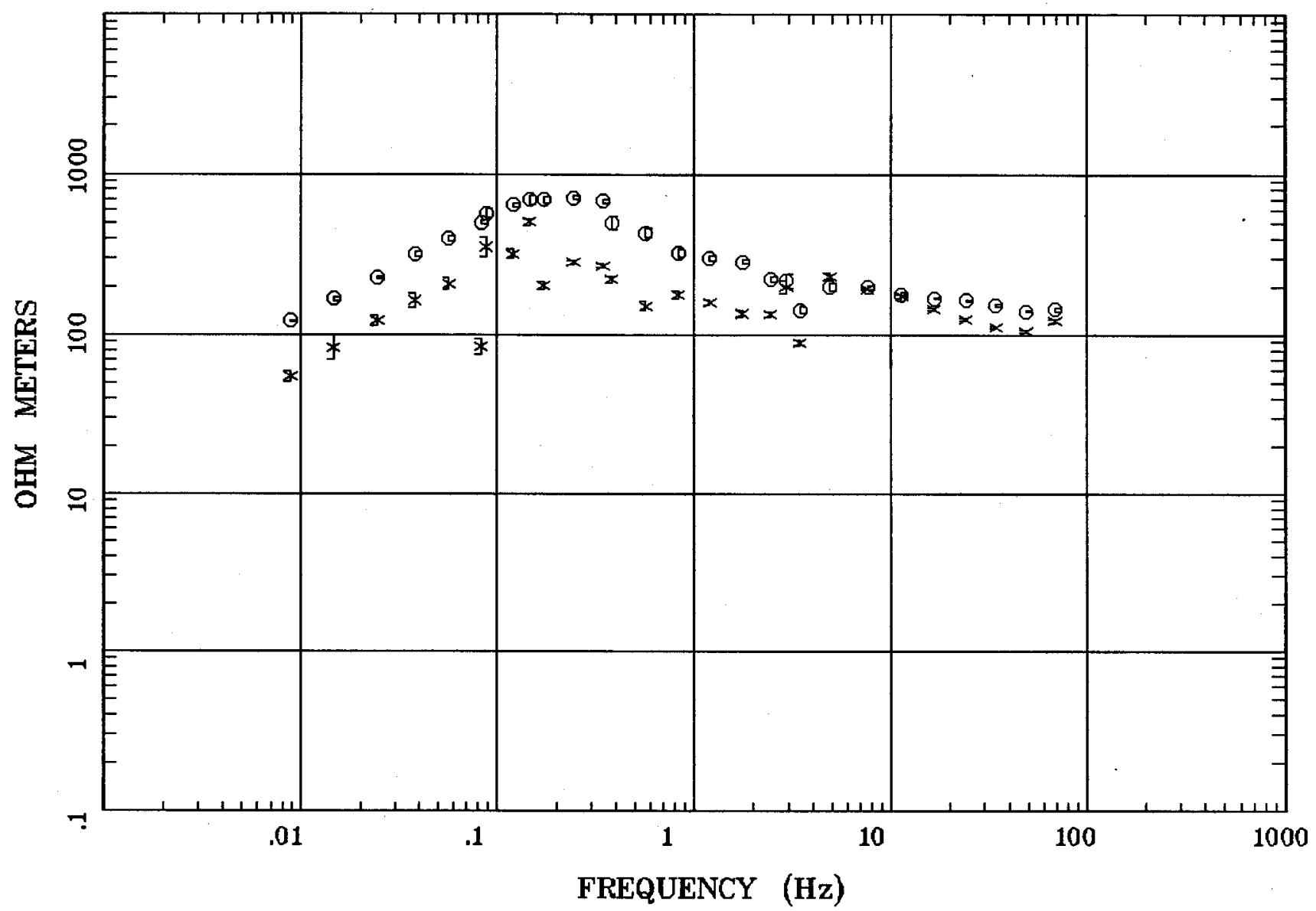

Client: WAter Resources \& DOD

Rotation:

Filename: nt65m.avg

Channels: Ch1 Ch2 Ch3 Ch4 Ch5 Ch3 Ch4 Plotted: 11:12 Feb 09, 2006

Acquired: 04:2 oct 18, 2005 Survey Co:US Geological Survey

< EMI - ElectroMagnetic Instruments > 


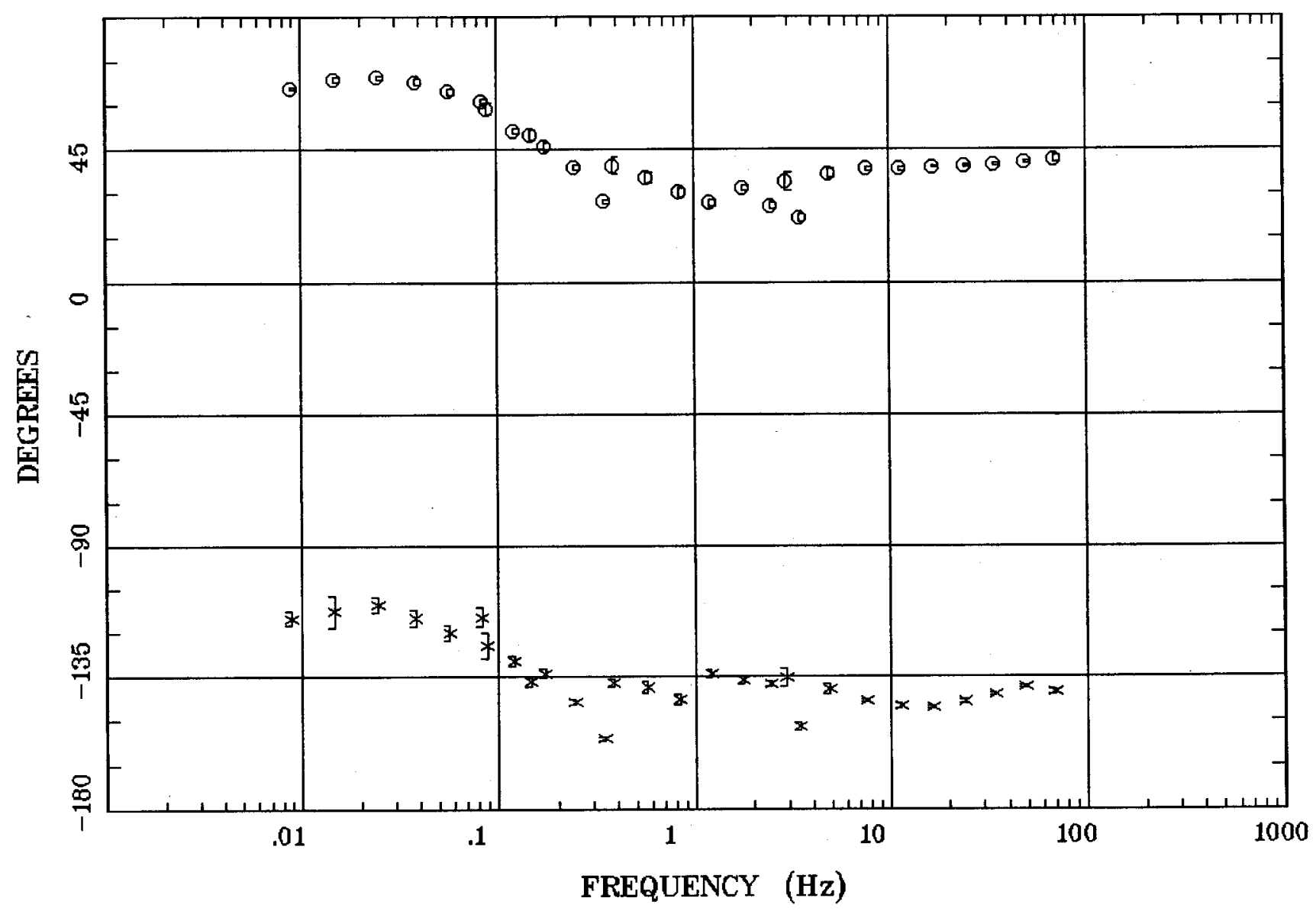

Client: WAter Resources \& DOD Remote: none Acquired: 04:2 oct 18, 2005 Survey Co:US Geological Survey
North of Raineer Mesa Road

\section{Rotation:}

Filename: nt65m.avg Channels: Ch1 Ch2 Ch3 Ch4 Ch5 Ch3 Ch4 Plotted: 11:12 Feb 09, 2006

< EMI - ElectroMagnetic Instruments > 


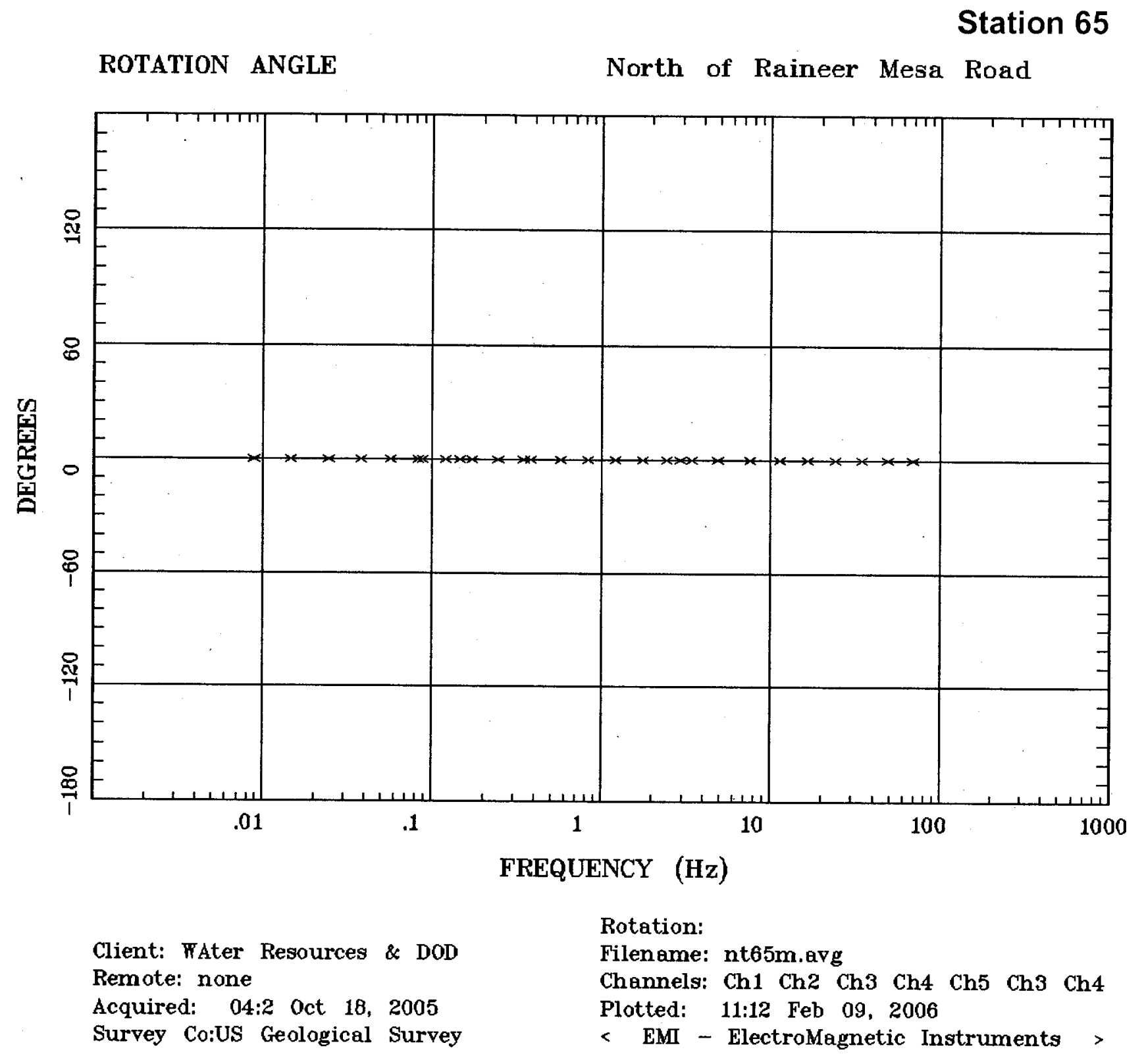




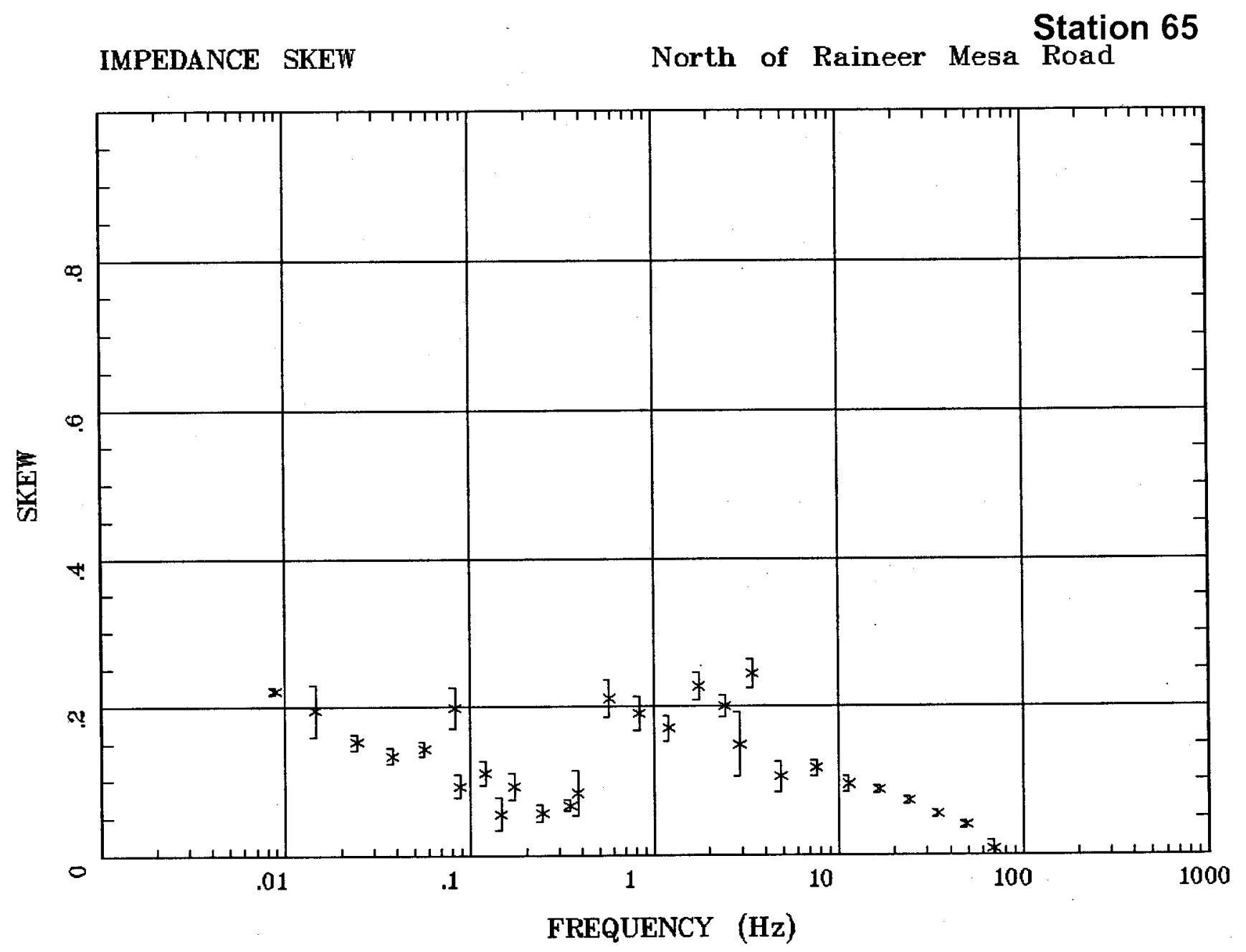

Client: WAter Resources \& DOD

Rotation:

Remote: none

Filename: nt65m.avg

Channels: Ch1 Ch2 Ch3 Ch4 Ch5 Ch3 Ch4

Plotted: 11:12 Feb 09, 2006

Acquired: 04:2 oct 18, 2005

Survey Co:US Geological Survey

< EMI - ElectroMagnetic Instruments > 
E MULT Coh.

Station 65

North of Raineer Mesa Road

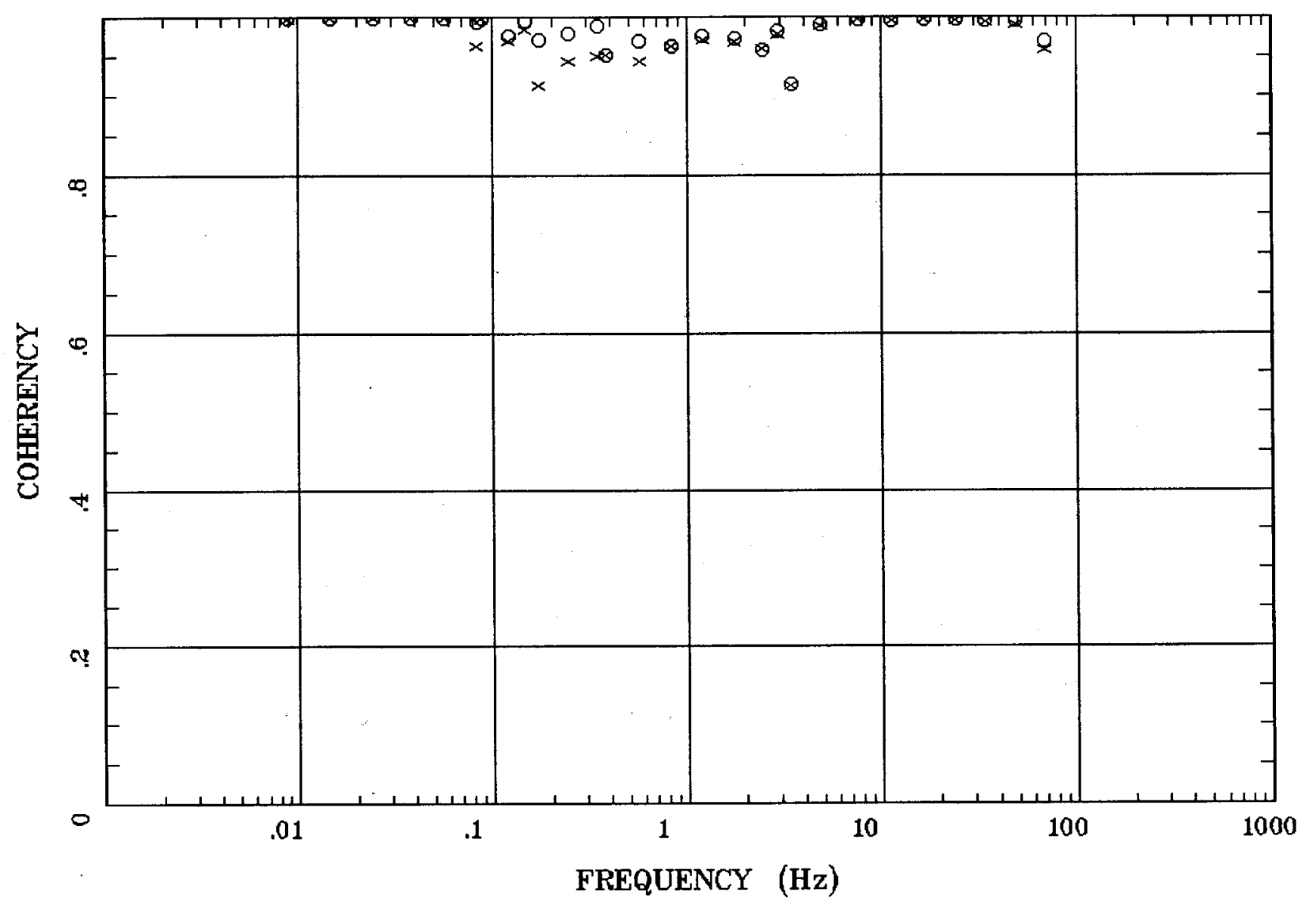

Client: WAter Resources \& DOD Remote: none Acquired: 04:2 oct 18, 2005 Survey Co:US Geological Survey
Rotation:

Filename: nt65m.avg Channels: Ch1 Ch2 Ch3 Ch4 Ch5 Ch3 Ch4 Plotted: 11:12 Feb 09, 2006

< EMI - ElectroMagnetic Instruments > 


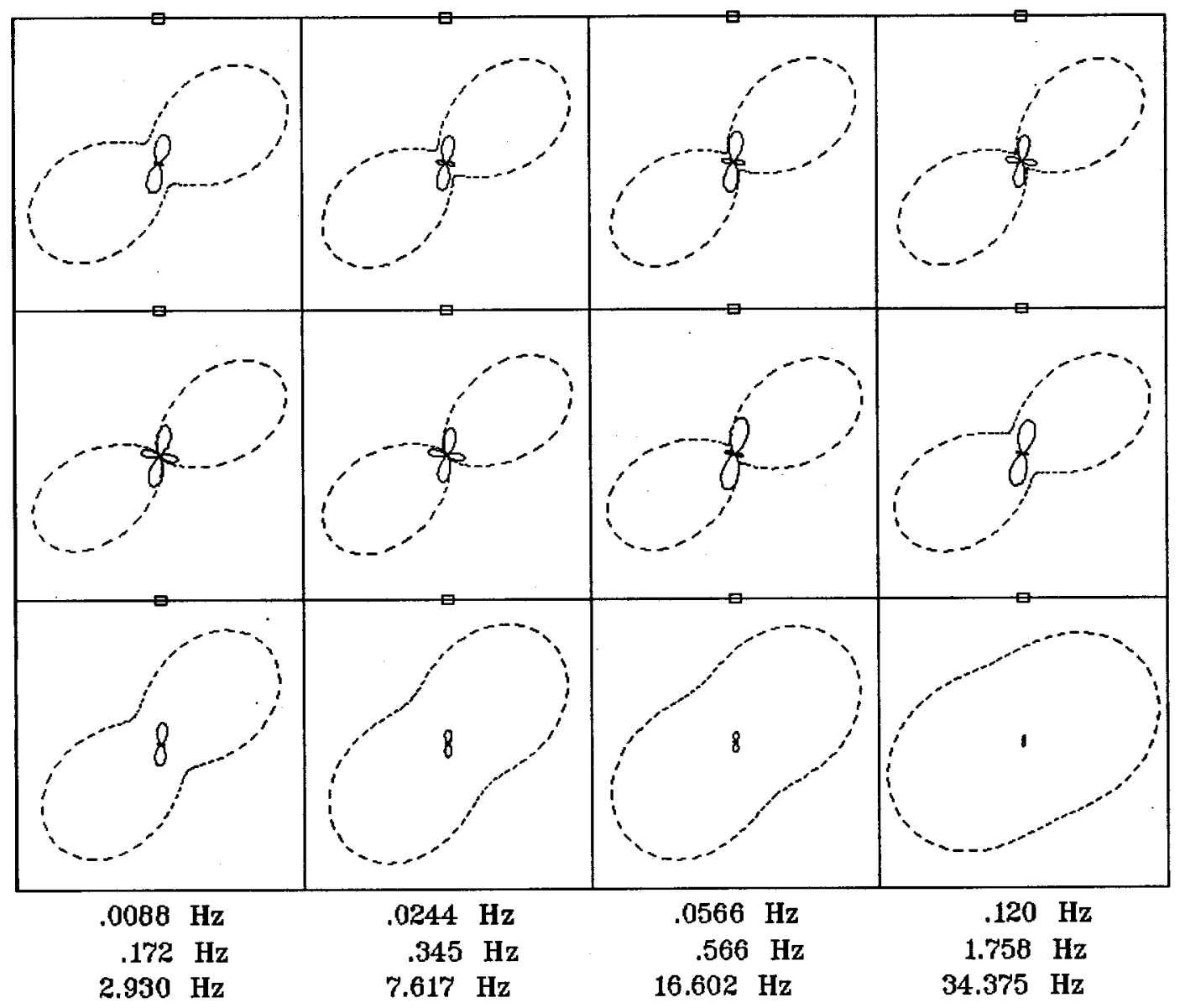

Client: FAter Resources \& DOD Remote: none

Acquired: 04:2 oct 18, 2005 Survey Co:US Geological Survey
Rotation:

Filename: nt65m.avg Channels: Ch1 Ch2 Ch3 Ch4 Ch5 Ch3 Ch4 Plotted: 11:12 Feb 09, 2006

< EMI - ElectroMagnetic Instruments > 
TIPPER MAGNITUDE

Station 65

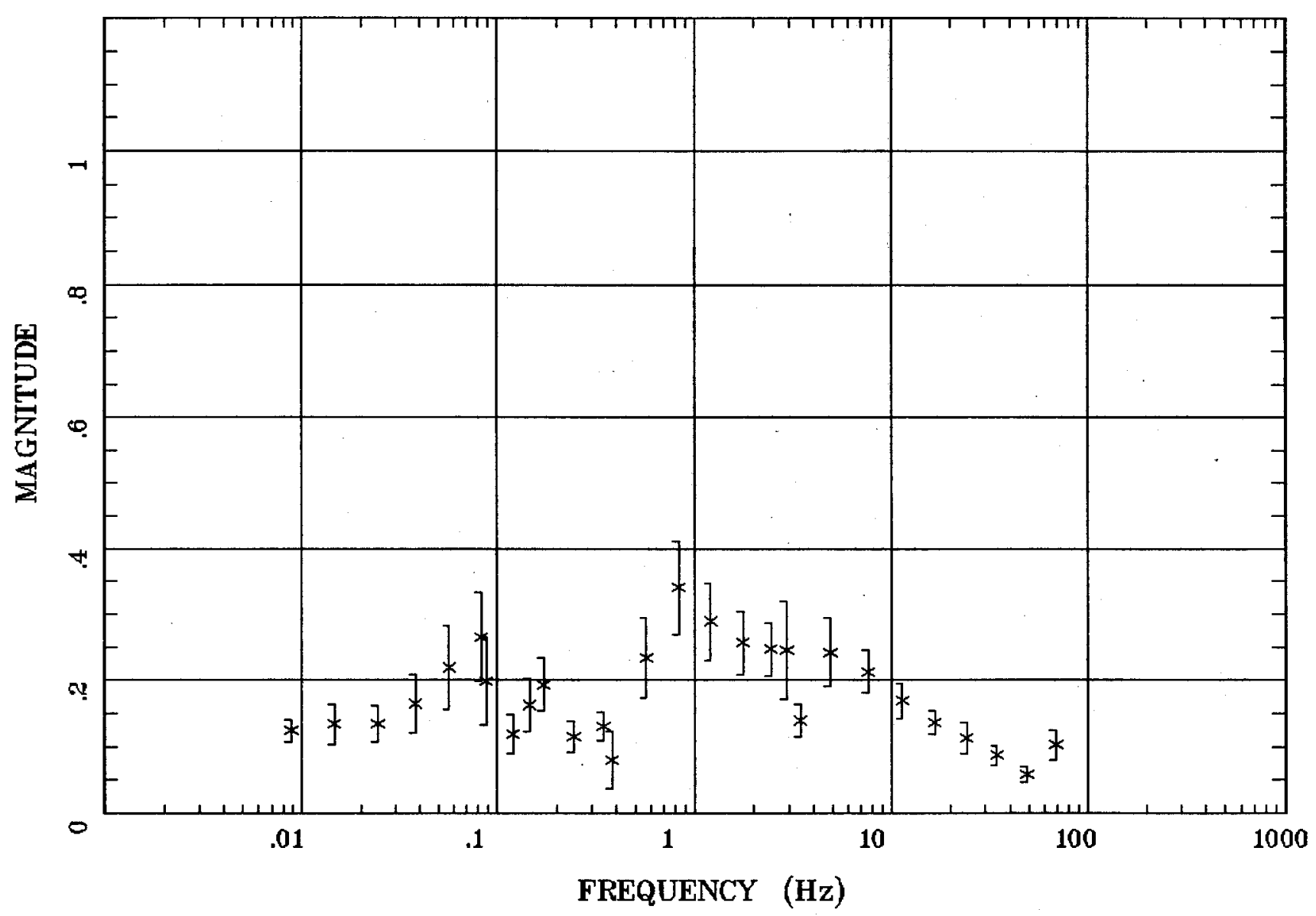

Client: WAter Resources \& DOD Remote: none

Acquired: 04:2 oct 18, 2005 Survey Co:US Geological Survey

Rotation:

Filename: nt65m.avg

Channels: Ch1 Ch2 Ch3 Ch4 Ch5 Ch3 Ch4. Plotted: 11:12 Feb 09, 2006

< EMI - ElectroMagnetic Instruments > 
TIPPER STRIKE

Station 65

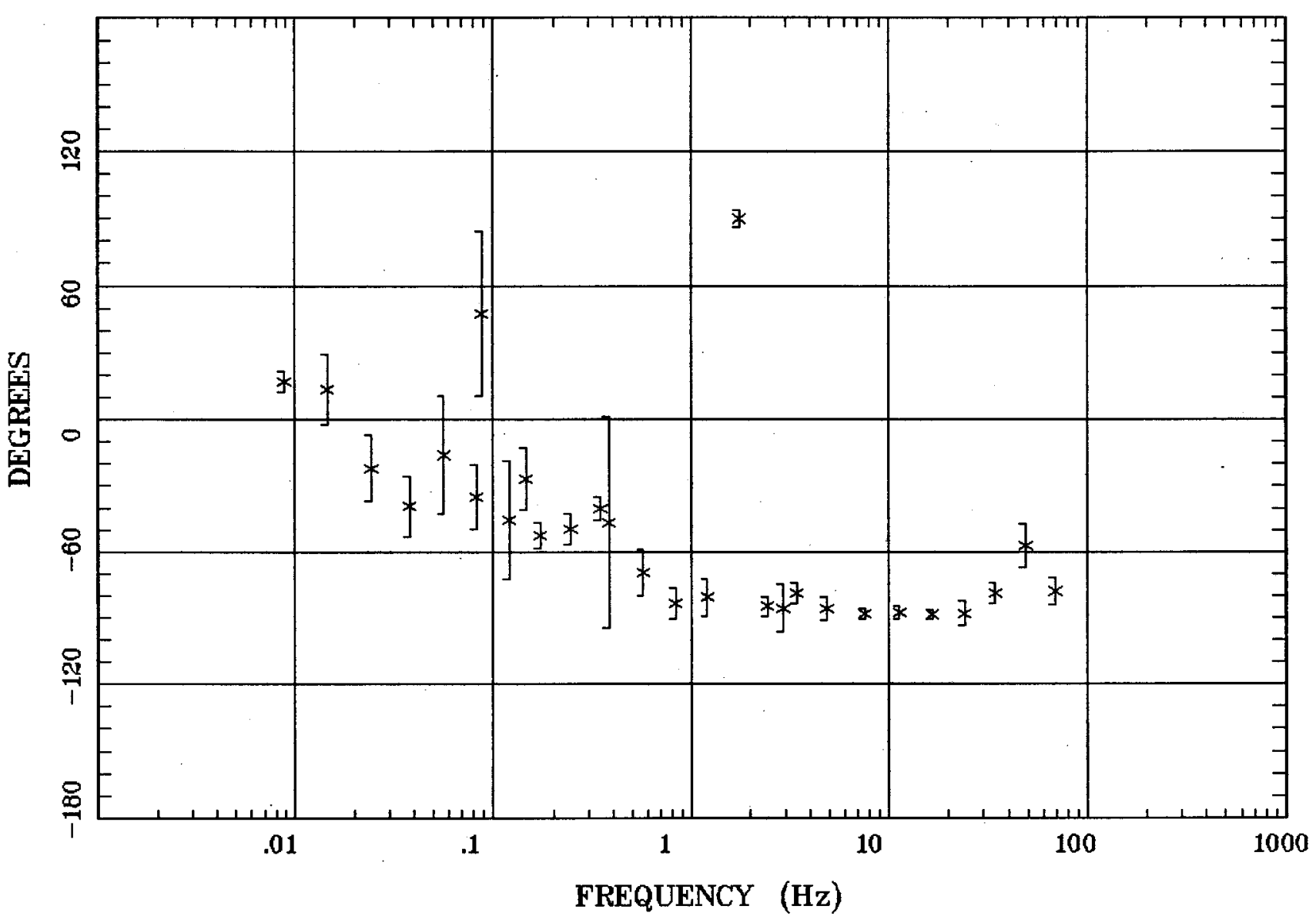

Client: WAter Resources \& DOD

Rotation:

Remote: none

Filename: nt65m.avg

Channels: Ch1 Ch2 Ch3 Ch4 Ch5 Ch3 Ch4

Acquired: $04: 2$ oct 18,2005

Survey Co:US Geological Survey

Plotted: 11:12 Feb 09, 2006

< EMI - ElectroMagnetic Instruments > 
Station 65

HzHx.x Coh HzHy.o

North of Raineer Mesa Road

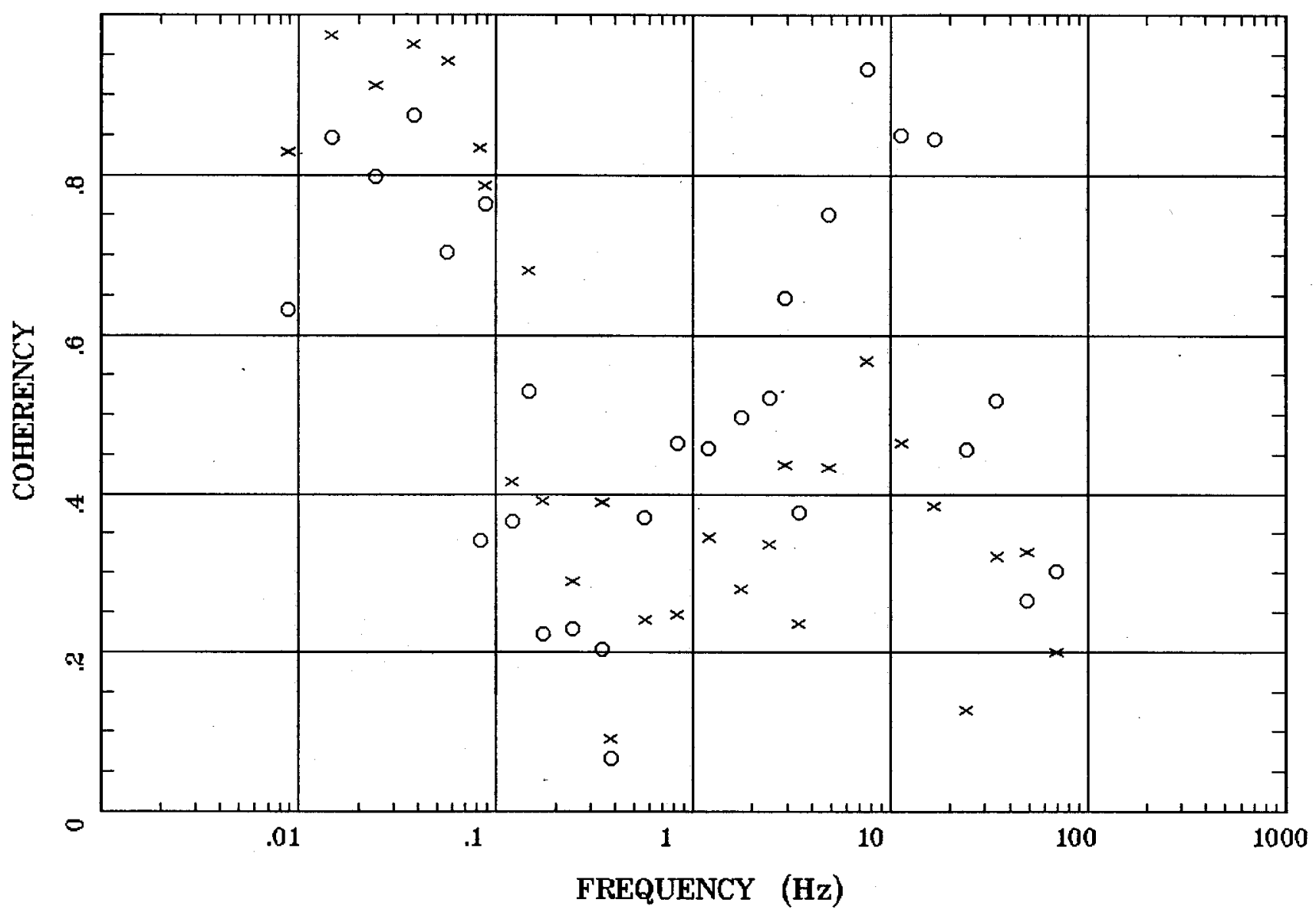

Client: WAter Resources \& DOD

Rotation:

Remote: none

Filename: nt65m.avg

Channels: Ch1 Ch2 Ch3 Ch4 Ch5 Ch3 Ch4

Acquired: 04:2 oct 18, 2005

Platted: 11:12 Feb 09, 2006

Survey Co:US Geological Survey

< EMI - ElectroMagnetic Instruments > 